\title{
Food reward from a behavioural and (neuro)physiological perspective
}

Suzanne E.M. de Bruijn 


\section{Thesis committee}

\section{Promotors}

Prof. Dr C. de Graaf

Professor of Sensory Science and Eating Behaviour

Wageningen University \& Research

Prof. Dr R.F. Witkamp

Professor of Nutrition and Pharmacology

Wageningen University \& Research

\section{Co-promotor}

Dr G. Jager

Associate professor, Division of Human Nutrition

Wageningen University \& Research

\section{Other members}

Prof. Dr B.J.M. Witteman, Wageningen University \& Research

Prof. Dr T. Kirkham, University of Liverpool, UK

Dr D.J. Mela, Unilever R\&D, Vlaardingen

Dr L. Steenbergen, University of Amsterdam

This research was conducted under the auspices of the Graduate School VLAG (Advanced studies in Food Technology, Agrobiotechnology, Nutrition and Health Sciences). 


\title{
Food reward from a behavioural and (neuro)physiological perspective
}

\author{
Suzanne E.M. de Bruijn
}

Thesis

submitted in fulfilment of the requirements for the degree of doctor at Wageningen University

by the authority of the Rector Magnificus,

Prof. Dr A.P.J. Mol,

in the presence of the

Thesis Committee appointed by the Academic Board

to be defended in public

on Friday 13 October 2017

at $1.30 \mathrm{p} . \mathrm{m}$. in the Aula. 
Suzanne E.M. de Bruijn

Food reward from a behavioural and (neuro)physiological perspective 156 pages.

$\mathrm{PhD}$ thesis, Wageningen University, Wageningen, the Netherlands (2017)

With references, with summaries in English and Dutch

ISBN: 978-94-6343-674-8

DOI: $\quad 10.18174 / 421669$ 


\section{Table of contents}

Chapter 1

General introduction

Chapter 2

The reliability and validity of the Macronutrient and Taste Preference Ranking Task: a new method to measure food preferences

Chapter 3

Explorative placebo-controlled double-blind intervention study with low doses of inhaled THC and CBD reveals no effect on sweet taste intensity perception and liking in humans

Chapter 4

No differences in satiety hormone and endocannabinoid response to palatable vs neutral food intake

Chapter 5

Decreased preference for high-carbohydrate and high-fat foods after Roux-en-Y gastric bypass surgery

Chapter 6

Altered neural responsivity to food cues in relation to food preferences, but not appetiterelated hormone concentrations after RYGB surgery

Chapter 7

Altered neural inhibition responses to food cues after Roux-en- $Y$ gastric bypass

Chapter 8

General discussion

References

Et cetera

Summary

Samenvatting

Dankwoord

About the author

List of publications

Overview of completed training activities 


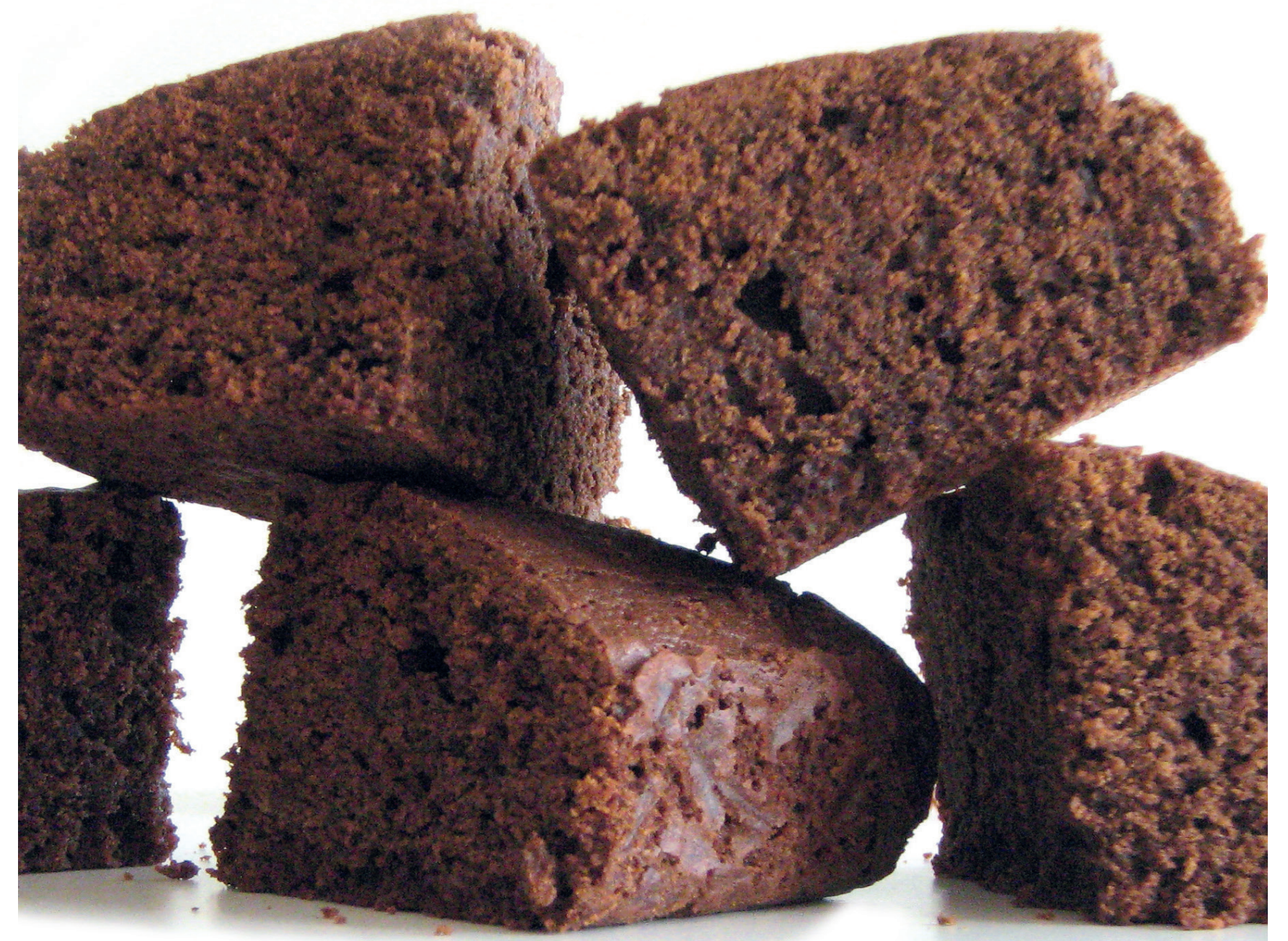




\section{CHAPTER 1}

GeNerAL INTRODUCTION 


\section{Background}

The mere sight and smell of foods can trigger a response of our reward system, both on a hormonal and on a neural level (Smeets et al., 2010). Moreover, seeing and smelling a food increases desire to eat that specific type of food (Fedoroff et al., 2003; Ramaekers et al., 2016). High-energy, palatable foods can trigger food intake even in the absence of energy deficits. This food consumption for pleasure, mostly referred to as hedonic eating, can easily cause individuals to overeat and may therefore contribute to the development of obesity (Fulton, 2010). Given the rise in obesity rates and the health risks associated with being obese, hedonic eating and food reward are receiving considerable interest from the scientific community, with different disciplines being involved.

Hedonic eating and food reward are highly complex biological processes, and many questions are still unresolved. Important factors to investigate include food choice behaviour and physiological responses to foods in healthy individuals. Furthermore, changes in behavioural and (neuro)physiological responses in patients who underwent gastric bypass surgery can provide more insight into alterations in food reward.

The aim of this thesis is to add on to the existing knowledge on food reward. To this end, the phenomenon was approached from a behavioural and (neuro)physiological perspective in healthy and in gastric bypass populations. This introduction starts with a description of food reward, with a focus on three different perspectives: 1) behavioural, 2) physiological, and 3) neurophysiological. Focus will then be on changed food reward in obesity and the most effective treatment of obesity: gastric bypass surgery. Lastly, the aim and thesis outline are described.

\section{Food reward}

Food reward is elicited by, and fluctuates during the food intake cycle, which is often divided into an anticipatory, consummatory, and post-ingestive phase (see Figure 1.1; Kringelbach et al., 2012). Food anticipation occurs at regular eating times, that is, right before usual dinner time, and in response to the sight and smell of foods. Upon food consumption, the taste and mouth feel of a food are sensed. Post-ingestive consequences, related to the energy- and nutrient-content of a food, are noticeable after food consumption. Both the taste of a food and the post-ingestive consequences facilitate a learning process that steers food preferences and reward elicited by anticipation of foods (Kringelbach et al., 2012; Smeets et al., 2010).

It is important to note that food reward comprises both food 'wanting' and food 'liking' (Berridge, 1996). Food wanting corresponds with desire to eat, or motivation to obtain a food, whereas food liking corresponds with a palatability, or pleasantness, response to a food (Berridge, 1996; Mela, 2006). In animal studies, wanting was found to be predominantly related to dopamine activity in the mesocorticolimbic system. Liking is currently more considered to be related to opioid activity in limbic brain areas, including the nucleus accumbens shell (Havermans, 2011). Importantly, other mediators such as endocannabinoids and gut hormones also influence food reward (see e.g. Bewick, 2012; Jager and Witkamp, 2014; Kroemer et al., 2013). 


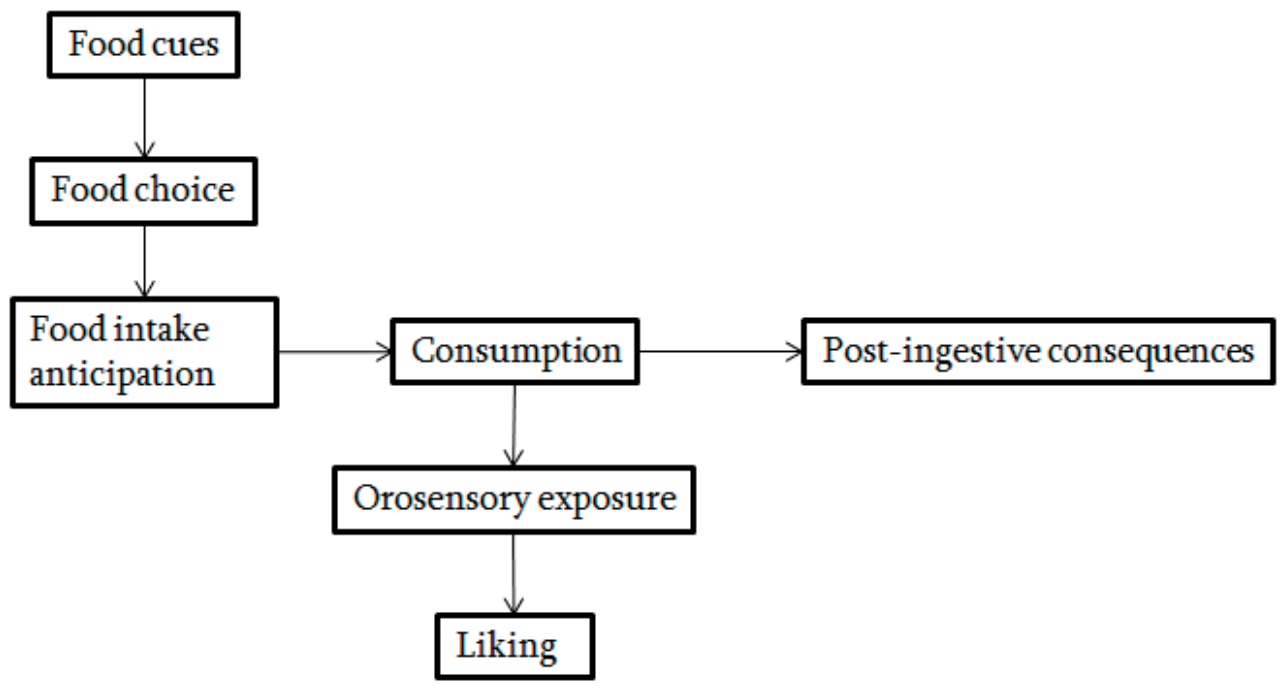

Figure 1.1. Schematic overview of the food intake cycle (Based on Blundell et al., 2010; Kringelbach et al., 2012). Each stage of food intake, that is anticipation, consumption and post-ingestion, can elicit food reward.

\section{Behavioural perspective: food preferences}

An easy method to gain insight into food 'wanting' and food 'liking' is to use food preference questionnaires. Other methods include computerized food preference tasks.

In food preference questionnaires, participants are asked to rate how much they like or how much they desire to eat different foods. The number of foods included in such questionnaires ranges from a small selection of specific foods to a wide range of over 300 food products to assess preferences for different food groups (Frank and Van der Klaauw, 1994; Geiselman et al., 1998). An example of a computerized task is the Leeds Food Preference Questionnaire (LFPQ; Finlayson et al., 2007). In the LFPQ participants are forced to choose their preferred food between two food products from different categories, high- or low-fat and sweet or savoury. Other studies adopted different food categories, that is, high- or low-protein and sweet or savoury, or high- or low-energy and sweet or savoury (Griffioen-Roose et al., 2011; Ijpma et al., 2016; Zoon et al., 2014). At the start of this thesis, it was not possible to assess and compare preferences for a full range of macronutrients in one task (Ijpma et al., 2016), whereas this was considered essential for our studies. Therefore, in the current thesis, a new food preference task that includes multiple food categories and a forced-choice procedure was developed. This task was then used to assess food preference under different circumstances.

\section{Physiological perspective}

Anticipation of food intake triggers a so-called cephalic phase response (CPR), which serves to prepare the body to optimally digest and absorb nutrients (Smeets et al., 2010). Anticipation of 
food intake can be triggered by the sight or smell of food, but also by learned triggers such as the time of day (Mattes, 1997). Several responses to anticipated food intake, such as salivation, gastric mobility and insulin release have been described (Mattes, 1997; Power and Schulkin, 2008; Smeets et al., 2010). In addition, exposure to the sight of food triggers a rise in ghrelin concentrations (Rigamonti et al., 2015; Schussler et al., 2012). Ghrelin is the only known orexigenic hormone, with levels rising in anticipation of food intake, and dropping after food intake (Cummings et al., 2001). Upon food entering the mouth, salivation increases more (Mattes, 1997) and mastication leads to increased concentrations of peptides such as insulin and pancreatic polypeptide (PP; Teff et al., 1991; Witteman et al., 1994).

Often, modified sham-feeding is used as a method to study the CPR. In modified shamfeeding, a food is chewed on, but instead of being swallowed, it is expectorated (Teff et al., 1991). Studies using modified sham-feeding have shown an effect of macronutrient content on release of different satiety hormones like PP (Witteman et al., 1994) and ghrelin (Zhu et al., 2014). Moreover, palatability has been found to modulate responses of PP and the endocannabinoid 2arachidonoylglycerol (2-AG; Mennella et al., 2015). At the start of this thesis, there was one published study that investigated the physiological response to actual food consumption of a palatable and a non-pleasurable food product. The results suggested a role for ghrelin and 2-AG in hedonic eating, or eating for pleasure, in humans (Monteleone et al., 2012). However, the sample size in that study was small $(\mathrm{n}=8)$ and the results might have been affected by expectations of food liking (Monteleone et al., 2012). This thesis extends the knowledge on the role of endocannabinoids, ghrelin and PP in food intake, by studying the response to consumption of palatable versus neutral foods where palatability was unknown until the first bite.

\section{Endocannabinoid system}

Endocannabinoids are part of the endocannabinoid system (ECS): a neuromodulatory system that comprises two cannabinoid receptors, $\mathrm{CB}_{1}$ and $\mathrm{CB}_{2}$, their endogenous ligands, the endocannabinoids of which anandamide (AEA) and 2-AG are best known, and the enzymes responsible for synthesis and degradation of the endocannabinoids (Howlett, 2002). The ECS plays an important role in food reward (Jager and Witkamp, 2014; Piazza et al., 2017). However, most studies into the role of the ECS in food reward have been performed in rodents. In animals, endocannabinoid stimulation enhances motivation to obtain palatable foods (Droste et al., 2010; Higgs et al., 2003) and liking of sweet and fatty foods (De Luca et al., 2012; O'Brien et al., 2013). Vice versa, endocannabinoid inhibition reduces liking of palatable foods (Droste et al., 2010; Higgs et al., 2003; O'Brien et al., 2013) and decreases total food intake (Chambers et al., 2007; Farrimond et al., 2012). Anecdotal evidence suggests that the use of Cannabis preparations increases food palatability and intake in humans (Abel, 1975; Foltin et al., 1988; Hart et al., 2002; Smit and Crespo, 2001; Tart, 1970), a phenomenon colloquially known as 'the munchies'. Furthermore, the $\mathrm{CB}_{1}$ inverse agonist rimonabant decreases food intake (Pi-Sunyer et al., 2006; Van Gaal et al., 2005) and neural activation in reward-related brain areas in response to palatable food exposure (Horder et al., 2010). To extend our knowledge on the role of the ECS in food reward in humans, effects of ECS 
modulation on sweet taste intensity perception and liking, and food preferences are investigated in this thesis.

\section{Neurophysiological perspective: brain imaging}

Neurophysiological responses can also provide more insight into underlying mechanisms of food reward and therefore brain imaging studies have been performed. Often used paradigms are food versus non-food viewing paradigms (see e.g. Killgore et al., 2003; LaBar et al., 2001), which are suggested to signal food anticipation (McCrickerd and Forde, 2016). In general, these studies find increased activation in reward-related brain areas, including the orbitofrontal cortex, striatum and insula, for viewing of foods over non-foods. In addition, looking at pictures of high-energy foods elicits more activation than looking at pictures of low-energy foods (van der Laan et al., 2011).

Other studies used odours to signal food anticipation. In these studies, an olfactometer was used to deliver specific odours for short periods of time, that is, seconds. Similar to looking at pictures, smelling food odours elicits more reward-related activation than smelling non-food odours (Frasnelli et al., 2015; Jiang et al., 2015).

\section{Neurophysiology in obesity}

Brain imaging studies showed increased activation in response to food in reward-related brain areas in obese compared to lean individuals (Patriarca et al., 2017), and it has often been suggested that obesity is the result of dysfunctional food reward processing (see e.g. Davis et al., 2004; Nasser, 2001). This seems to be underlined by observations that dopamine signalling is impaired in obese individuals (Kroemer and Small, 2016). However, the functional outcomes of this impaired dopamine signalling remain debated.

Some studies suggest that decreased dopamine signalling causes a lower reward response, that is, hyposensitivity to reward (Volkow et al., 2008). Obese individuals may increase their food intake to compensate for a reward deficit (Kenny, 2011; Stice et al., 2008a). Others suggest that obese individuals are hypersensitive to reward. The so-called reinforcement sensitivity model poses that obese individuals are more responsive to reward and therefore increase their food intake (Davis et al., 2004). Both models, that is, hypo- and hypersensitivity to reward, account for the hypothesis that obese individuals have a higher motivation to obtain food rewards (Mela, 2006), either to compensate for a reward deficit or as a result of increased reward sensitivity. In support of this hypothesis, it has been shown that willingness to work for a high-energy food reward is increased in obese compared to lean individuals (Giesen et al., 2010; Saelens and Epstein, 1996). In addition, attention for foods is increased in obese compared to lean individuals (Doolan et al., 2014; Hendrikse et al., 2015; Nijs et al., 2010). Importantly, obese individuals show a bias towards both high- and low-energy foods (Kemps and Tiggemann, 2015).

\section{Learnings from Roux-en-Y gastric bypass surgery}

Several studies suggest that weight loss induces changes in food preferences. In general, preference seem to shift away from highly palatable sweet and fatty foods to foods with lower sugar and lower fat content (Burgess et al., 2016; Ledikwe et al., 2007; Martin et al., 2011). However, not 
all studies found a change (see for a review Mathes and Spector, 2012). Importantly, motivation to obtain food rewards is not changed after a weight-loss intervention (Cameron et al., 2008), but it is decreased after Roux-en-Y gastric bypass (RYGB) surgery (Miras et al., 2012).

RYGB is currently regarded the most effective treatment to lose weight and to maintain weight loss (Buchwald et al., 2004; Gloy et al., 2013; Sjöström, 2013). After RYGB, patients often report decreased intake of foods high in carbohydrate and fat (Laurenius et al., 2013). As similar food intake behaviour has been found in animal studies (le Roux et al., 2011), this change in eating behaviour is likely caused by the effects of RYGB surgery, and not by dietary advice. In RYGB surgery, the stomach size is decreased by creating a small gastric pouch, which is then connected to the jejunum. After surgery, food bypasses the duodenum and proximal part of the jejunum (Hng and Ang, 2012). Both the smaller stomach and the bypass induce weight loss by lowering food intake and reducing absorption from the gastrointestinal tract (Ward and Prachand, 2009). Perhaps even more important for the effects of RYGB on food intake behaviour are changes in gut hormone responses (Pedersen, 2013). This is most likely related to the fact that receptors and feed-back mechanisms in the duodenum are no longer triggered by nutrients following food intake.

Besides the reported changes in food choice, patients also indicate lower preference for high-energy foods after RYGB (Behary and Miras, 2015; Pedersen, 2013). When patients who underwent surgery are asked to indicate their desire to eat different foods, they indicate that desire to eat high-energy foods is decreased compared to the situation before surgery (Hansen et al., 2016; Meillon et al., 2013). This is underlined by changes observed in brain reward activation in response to food pictures (Ochner et al., 2012b).

Although several studies assessed food intake, food liking and desire to eat after RYGB (Bueter et al., 2011; Cushing et al., 2013; Kruseman et al., 2010; Ochner et al., 2012b), no studies have assessed food choice in a forced-choice paradigm. The advantage of a forced-choice procedure is that it provides more insight into food preferences. An individual might like two foods equally, but show a preference when forced to choose between the two foods. The relative preference for one food over another can provide insight into motivation for the chosen food (Finlayson et al., 2007; Finlayson et al., 2008). In this thesis, a ranking paradigm was used to assess food preferences before and after RYGB. Moreover, to gain insight into the mechanisms behind the behavioural changes, endocannabinoid and ghrelin plasma concentrations were assessed before and after surgery. We also report brain activation in response to viewing and engaging with high- and lowenergy food pictures and odours.

\section{Aims and thesis outline}

To better understand the changes in food preferences and food reward after weight loss and RYGB surgery, it is necessary to have in-depth understanding of the behavioural and (neuro)physiological mechanisms underlying food reward in healthy weight individuals. Such knowledge can guide research into altered food preferences and food reward. Eventually, a better understanding of the mechanisms behind changed food preferences might guide novel non-surgical interventions that are less invasive while bearing lower risks of complications. 
Therefore, the overall aim of this thesis is to gain more insight into food reward by investigating it from a behavioural and (neuro)physiological perspective in healthy weight and in gastric bypass populations. To achieve this, we aim to answer three questions;

1) Is it possible to develop a method to assess food preferences for four macronutrient and two taste categories?

2) To what extent does modulation of the endocannabinoid system lead to measurable changes in food reward and hedonic eating in humans in an experimental setting and, vice versa, is it possible to measure peripheral (plasma) responses of the endocannabinoid system to palatable food intake from anticipation until two hours after consumption?

3) What are the effects of Roux-en-Y gastric bypass surgery on food preferences and food reward?

In chapter 2 a newly developed food preference task, the Macronutrient and Taste Preference Ranking Task (MTPRT), is described and the results of a validation study are shown. The MTPRT is the first task that employs a ranking procedure with pictures of food products to measure food preferences for four macronutrient categories, that is, carbohydrate, fat, protein and low energy, and for two tastes, that is, sweet and savoury. The MTPRT was used in chapters 3,5 and 6 in this thesis. In the study described in chapter 3 , the aim is to determine whether stimulation and inhibition of the ECS modulate sweet taste intensity perception and liking. By using the MTPRT, effects of the treatments on food preferences were explored. In chapter 4, focus lays on the physiological response to consumption of a palatable and a neutral brownie. To this end, a complete profile of endocannabinoids, ghrelin and PP responses is measured during anticipatory, consummatory and post-ingestive phases up to two hours after ingestion.

In chapters 5, 6 and 7, focus is on changes in food preferences and food reward after RYGB surgery. In chapter $\mathbf{5}$, the aim is to assess food preferences before, two months after and one year after RYGB. To this end, participants complete an online version of the MTPRT at the mentioned time points. In chapter 6 , the underlying mechanisms of altered food preferences after RYGB are studied. Changes in reward-related brain activity in response to food odours and pictures are investigated, and a potential role for endocannabinoids and ghrelin in these changes is explored. In chapter 7, a food-specific go/no-go task is used to assess changes in neural responsivity during response inhibition.

Lastly, the collective findings of these studies, methodological considerations and suggestions for future research are discussed in the general discussion. 


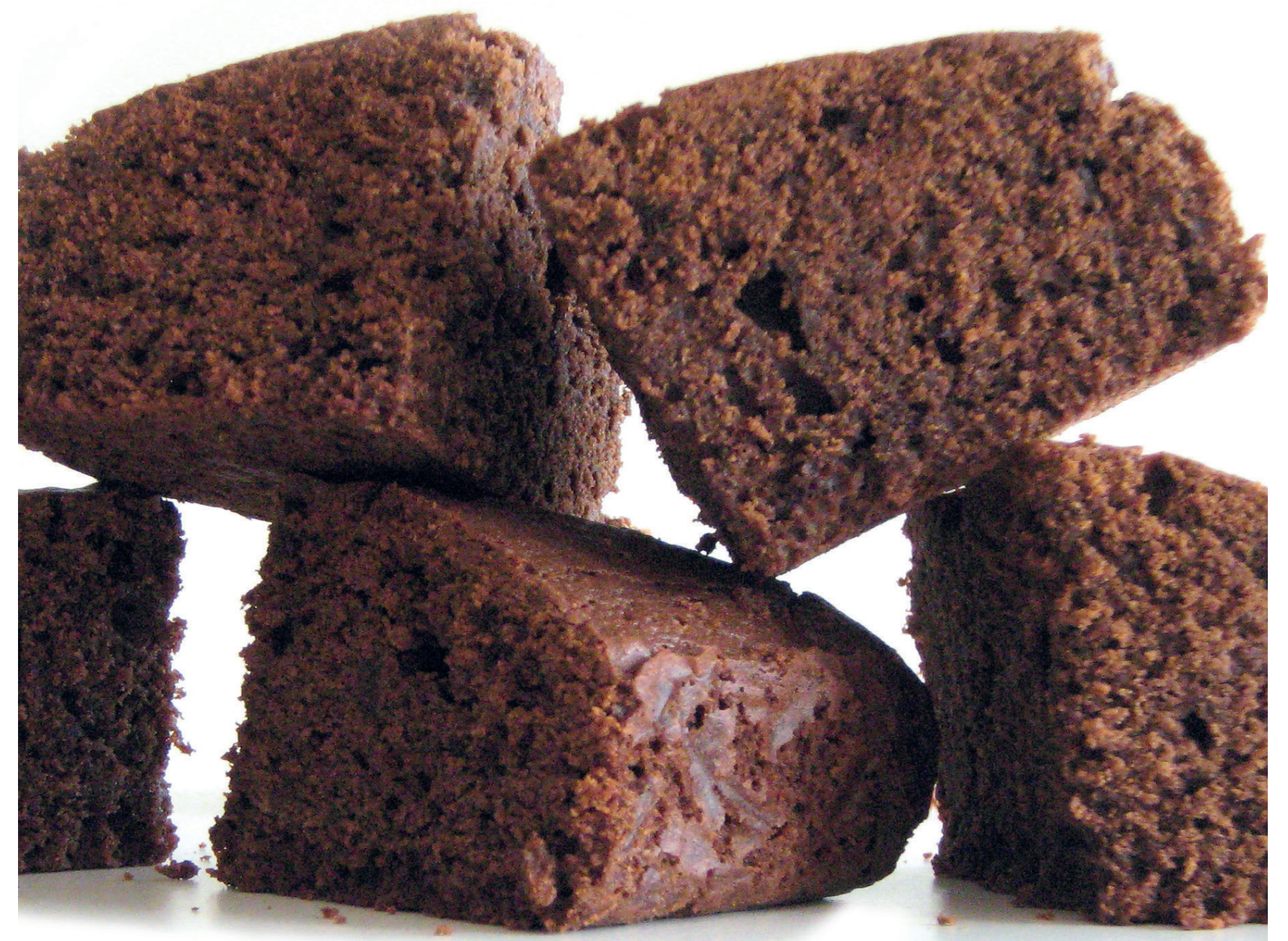




\section{CHAPTER 2}

\section{The rellability and validity of the Macronutrient and Taste Preference RANKING TASK: A neW METHOd tO MEASURE FOOd PREFERENCES}

Suzanne E.M. de Bruijn*

Yfke C. de Vries*

Cees de Graaf

Sanne Boesveldt

Gerry Jager

"Both authors contributed equally 


\begin{abstract}
Food preferences are for a large part determined by the macronutrient content and taste of foods, but may change depending on internal and external factors. Here, we discuss a newly developed food preference task, the Macronutrient and Taste Preference Ranking Task (MTPRT), in which participants rank groups of four food products according to how much they desire to eat the products. The MTPRT includes pictures of sweet and savoury food products from four categories: high-carbohydrate, high-fat, high-protein and low-energy. A within-subjects study on sensoryspecific satiety was conducted to assess the task's reliability and validity. Sixty-nine healthy participants performed two test sessions that were at least one week apart. Participants ate either a sweet or a savoury meal, which were similar in macronutrient content. Before and after eating the meal participants rated appetite and completed the MTPRT. In hungry state, preference scores for all food categories were significantly correlated between the two test sessions (all $\mathrm{r}>0.68$, all $\mathrm{p}<$ 0.001). Preference for sweet decreased after the sweet meal and increased after the savoury meal. In addition, preference for protein decreased more after consuming the savoury meal than it did after consuming the sweet meal. Preference for carbohydrate and fat decreased after meal consumption, regardless of taste. Preference for low-energy increased after meal consumption. These results show the MTPRT is a reliable and valid task for measuring food preferences. The MTPRT can be used for both hypothesis-driven and exploratory studies to examine the influence of different factors on changes in food preferences.
\end{abstract}




\section{Introduction}

Sensory properties of foods play an important role in the preferences for and intake of food (McCrickerd and Forde, 2016). The basic tastes seem to have specific signalling functions for the body, in that sweet taste signals carbohydrates, and salty and savoury taste signals protein and electrolytes (Scott, 2008). Indeed, various studies have demonstrated relations between sugar content and sweetness, and between protein content and salty and umami taste (Lease et al., 2016; Martin et al., 2014; Viskaal Van Dongen et al., 2012). The body uses these signalling cues to maintain macronutrient balance. Studies have shown that a protein-depleted state elicits a higher preference and reward for and intake of savoury foods in order to restore protein status (Griffioen-Roose et al., 2012b; Griffioen-Roose et al., 2014). Energy and macronutrient balance may be challenged in certain people, which may be related to changed preferences for foods. For instance after gastric bypass surgery preference for sweet and high-fat foods decreases (Miras et al., 2012; Pepino et al., 2014), while other studies report an increased preference for high-protein foods (Ullrich et al., 2013). In cancer patients undergoing chemotherapy, a reduced taste and smell function is frequently reported, which has consequences for food preferences and food intake (Boltong et al., 2014; Boltong et al., 2012; de Vries et al., 2016). However, it is important to note that also in the general population food preferences are influenced by many factors including the time of the day, the appropriateness of foods within a meal context and the meal eaten previously (Birch et al., 1984; De Graaf et al., 1993; Griffioen-Roose et al., 2010). This multitude of factors that influence food preferences makes measuring food preferences a challenge. To better understand how food preferences can shift in different situations, it is essential to include macronutrient and taste composition when measuring food preferences. However, few methods are available that capture both macronutrient and taste composition and that are able to assess shifts in food preferences by these factors.

A questionnaire that takes both macronutrient and taste composition into account is the macronutrient preference checklist (MPC). The MPC is a list of foods divided over four macronutrient categories, including both sweet and savoury products. Participants are instructed to check off all foods in the MPC that one would like to eat right at that moment (Brisbois-Clarkson et al., 2009; Hill 1986; Hill et al., 1987). This method results in frequencies of selected products from specific macronutrient or taste categories. Another method that includes different macronutrient and taste categories is the Leeds Food Preference Questionnaire (LFPQ; Finlayson et al., 2007). The questionnaire uses food pictures rather than words, which is of importance as visual cues are important factors in food selection and give input on the edibility, palatability and satiating properties of a food (McCrickerd and Forde, 2016). The LFPQ is a computer-based food preference task in which participants make forced choices between two food products from four different food categories. Thereby, products are not just rated on their own. Instead, products from different product categories are directly compared and relative preferences for food categories are measured. These relative preferences provide insight in motivation for the chosen food category over the nonchosen food category (Finlayson et al., 2008). However, the LFPQ includes only two macronutrientbased categories divided over sweet and savoury taste. In the original LFPQ Finlayson and colleagues used high- and low-fat foods (Finlayson et al., 2007; Finlayson et al., 2008). Later studies adapted this to include high- and low-protein (Griffioen-Roose et al., 2011) and to high- and low- 
energy (Zoon et al., 2014). As it is essential to be able to assess preferences for a full range of macronutrients, we developed the Macronutrient and Taste Preference Ranking Task (MTPRT), which includes both macronutrient and taste categories. The task was developed based on the following criteria 1) foods included should be from multiple macronutrient categories and tastes, 2) the foods should be presented as pictures rather than words, and 3) should consist of a ranking paradigm in order to assess relative food preferences.

The MTPRT consists of pictures of products from four macronutrient categories, i.e., highcarbohydrate, high-fat, high-protein and low-energy, including both sweet and savoury products. Participants are asked to make rankings of four products based on how much they desire to eat the different products at that moment. These rankings are used to assess relative preferences for the four macronutrient categories and the two tastes sweet and savoury. In the current study we aim to show that the MTPRT is a reliable and valid task to measure food preferences: to demonstrate reliability of the task, we assessed test-retest reliability. To demonstrate validity of the task, we assessed the discriminative ability of the task by assessing sensory-specific satiety; after eating a food to satiety, the pleasantness of sensory properties of that food is decreased more than of foods that have not been eaten (Rolls et al., 1981). Based on previous studies we expect preference for sweet products to decrease after a sweet test meal and to increase after a savoury test meal. Furthermore we expect decreased preferences for high-carbohydrate and high-fat products after eating a meal in general, a decreased preference for high-protein products after a savoury test meal and an increased preference for low-energy products after eating a meal in general.

\section{Materials and methods}

\section{Products and categories of the MTPRT}

A total of 32 food products from four macronutrient categories, i.e., high-carbohydrate, high-fat, high-protein and low-energy was used in the MTPRT. Each category contained eight products, of which four products were sweet and four were savoury. The high-protein category formed an exception and consisted of eight savoury products, as no products met all requirements to be included as high-protein sweet. A product had to meet the following requirements to be included in the MTPRT:

- Commercially available.

- High-fat, high-protein and high-carbohydrate foods contained at least $50 \%$ of total energy from their respective macronutrient category classification.

- Low energy products contained less than $60 \mathrm{kcal} / 100$ gram.

Hill's European MPC (Hill 1986; Hill et al., 1987) and Brisbois-Clarkson's North American MPC (Brisbois-Clarkson et al., 2009) were used as starting point to select the food products. Products were replaced based on commercial availability in the Netherlands when needed. The Dutch Food Composition table was used to ensure appropriate macronutrient composition (RIVM, 2011). The final list of products including their respective nutritional values can be found in Supplementary Table 2.1.

For the products that were included in the MTPRT, standardized pictures were provided by the Image Sciences Institute, UMC Utrecht, and created as part of the Full4Health project 
(www.full4health.eu), funded by the European Union Seventh Framework Program (FP7/20072013) under grant agreement $\mathrm{nr}$. 266408, and the I.Family project (http://www.ifamilystudy.eu), grant agreement nr. 266044 (Charbonnier et al., 2015b). Pictures of foods were standardized by means of the plate on which products are presented, background colour, contrast, camera distance and angle (see Fig. 2.1 for examples).

\section{Task procedure}

The MTPRT consisted of three parts: practicing, liking and ranking.

The practicing part was designed to familiarize participants with the ranking task. Participants were presented with four combinations of four pictures and asked to rank these pictures according to "what they most desire to eat at this moment". The pictures used in the practicing part were not used in the main task and did not necessarily fit within one of the macronutrient categories.

The liking part was designed to introduce participants to each product by name and picture. Liking was assessed by presenting pictures of all 32 products with the question: 'How much do you like [product name]?' which was rated on a 100 point visual analogue scale (VAS) anchored by 'do not like at all' and 'like extremely'.

The ranking part consisted of two sections, one focused on macronutrients the other on taste, i.e., sweet and savoury. In both sections, participants were presented with four different pictures, which they had to rank according to "what they most desire to eat at this moment" (Fig. 2.1). Participants first clicked on the product they most desired to eat at the moment of completing the task, then they clicked on the second most desired product, followed by the third and the product they least desired to eat at the moment of completing the task. In the macronutrient section, each of the four pictures represented one of the macronutrient categories. In total sixteen combinations of pictures were presented, in which each picture was shown twice. In the taste section, the four pictures that were presented came from two macronutrient categories. Within each category, one picture represented a sweet food item, and the other a savoury one. For example, one sweet product and one savoury product were high in carbohydrate and one sweet product and one savoury product were high in fat. In the taste section, products from the high-protein category were excluded, as this category only contained savoury products. In total twelve rankings were made in the taste section. As in the macronutrient section, each picture was shown twice.

For both sections, the order in which categories were presented on the screen was randomized and balanced across trials. For the macronutrient section, this meant that each macronutrient appeared four times on each of the four available positions. The same picture never appeared on the same position within this section. In the taste section, both tastes appeared six times on each of the four available positions. All pictures were presented twice, on two different positions.

The task took approximately 10 minutes to complete and was executed in E-Prime 2.0 (Psychology Software Tools, Pittsburgh, PA). The MTPRT can also be executed in EyeQuestion software (Logic8 BV), which facilitates participants to complete the task online. 
A

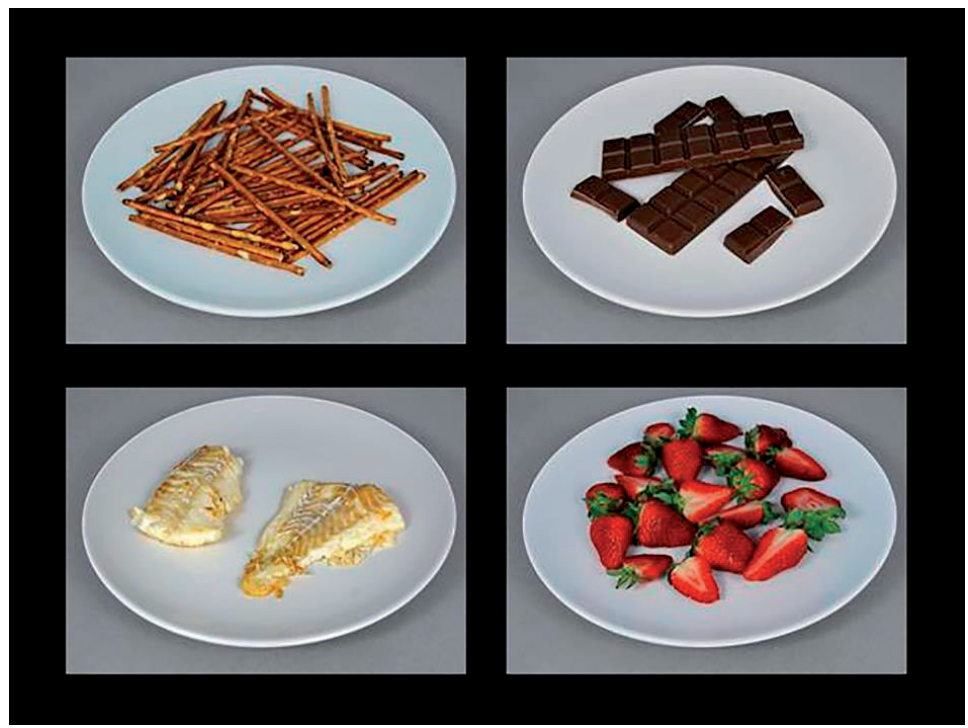

B

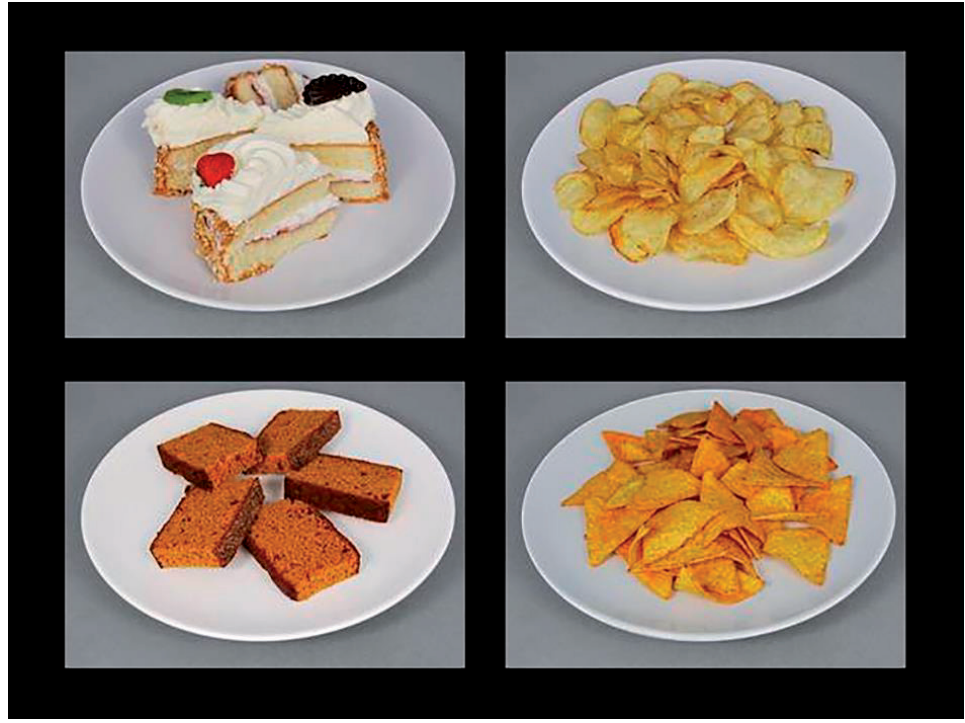

Figure 2.1. Lay-out of the macronutrient section (A) and taste section (B) of the MTPRT.

\section{Participants}

Healthy participants aged 18-35 years with a normal weight (BMI 18.5-25 kg/m²) were recruited. Exclusion criteria were restraint eating (DEBQ: men: >2.9, women $>3.4$; Van Strien et al., 1986), lack of appetite, difficulties swallowing or eating, having taste or smell disorders (self-report), energy restricted diet two months prior to the study, weight loss or gain $>5 \mathrm{~kg}$ during the two months prior to the study, being allergic or intolerant for the products under study, smoking, being vegetarian or vegan, and for women: being pregnant or lactating.

Seventy-four participants were included in the study. Five participants were excluded from the analyses because they did not comply with study procedures. Analyses were done on data of the 
remaining 69 participants ( 16 male/53 female) with an average age of 21.3 years $(\mathrm{SD}=2.9)$ and average BMI of $21.6 \mathrm{~kg} / \mathrm{m}^{2}$ ( $\left.\mathrm{SD}=1.8\right)$. All participants received a financial compensation for participating. The study was exempt from formal ethical approval by the Medical ethical Committee of Wageningen University. All participants signed an informed consent form.

\section{Study design}

We used a randomized crossover design, in which participants were invited to attend two test sessions that were at least seven days apart. Participants were told that the purpose of the study was to examine the effect of a sweet and savoury lunch on preference for different food products. During both test sessions, participants were presented with either a sweet or a savoury test meal, the order of which was randomized and balanced across the participants. When participants arrived they were instructed to first fill out an appetite questionnaire with 5 questions, i.e., hunger, fullness, prospective consumption, appetite for something sweet and appetite for something savoury. Questions were rated on a 100 point VAS anchored by 'not at all' and 'extremely', except for prospective consumption which was anchored by 'nothing at all' and 'a very large amount'. Next, participants completed the MTPRT, as described under 'task procedure'. After finishing the MTPRT, participants were presented with the test meal and $150 \mathrm{ml}$ water. They were instructed to finish the meal and water within 30 minutes. Next, participants rated the test meal for liking on a 100 point VAS anchored by 'not at all' and 'extremely' and completed the appetite ratings and MTPRT again.

To standardize hunger feelings for all participants in both test sessions, participants were instructed to standardize their breakfast and morning physical activity on both test days. They were asked to refrain from eating and to drink only water or tea without sugar during the three hours prior to the test session. Test sessions started at 11:30, 11:45, 12:00, 13:00, 13:15 and 13:30 and lasted approximately one hour. Both test sessions started at the same time for each individual participant.

\section{Test meals}

Participants received a standard amount of each test meal based on their energy requirements as calculated by the Schofield formula(Schofield, 1985) with a physical activity level of 1.6. The test meal contained $18 \%$ of participant's daily required energy intake, rounded to the nearest $50 \mathrm{kcal}$, which is $81 \%$ of the amount of energy provided by an average lunch in the Netherlands.(Hulshof et al., 2004)

Energy and macronutrient composition were similar for both meals and are shown in Table 2.1. The savoury meal consisted of risotto rice (Lassie, Wormer, The Netherlands) (65\%), semiskimmed milk (17\%), crème fraîche (11\%), bouillon $(0.3 \%)$, garlic powder $(0.02 \%)$, salt $(0.8 \%)$ and maltodextrin (Fantomalt, Nutricia, The Netherlands) (6\%). The sweet meal consisted of risotto rice (64\%), semi-skimmed milk (21\%), water (4\%), margarine (4\%), cinnamon $(0.08 \%)$, sucralose $(0.05 \%)$ and maltodextrin $(7 \%)$. A standard protocol was used to make fresh meals on each test day. Meals were kept warm with an average temperature of $81^{\circ} \mathrm{C}$ (range $69-89^{\circ} \mathrm{C}$ ). 
Table 2.1. Nutritional composition per $100 \mathrm{~g}$ of the sweet and savoury meal

\begin{tabular}{lll}
\hline & Sweet meal & Savoury meal \\
\hline Energy (kcal) & 89 & 91 \\
Energy (kJ) & 375 & 381 \\
Protein (g) & 1.9 & 2 \\
Fat (g) & 3.2 & 3.2 \\
Carbohydrates (g) & 13.1 & 13.2 \\
Fibre (g) & 0.2 & 0.2 \\
\hline
\end{tabular}

\section{Data analysis}

Each product received a macronutrient preference score based on the place the product was ranked. The higher the rank, the higher the score. Scores for all 16 presentations added up to a total score that was divided by the 16 times a product from each category was presented. Preference scores can range from 1 to 4 :

relative preference score macronutrient

$$
=\frac{4 *(\# \operatorname{rank} 1)+3 *(\# \operatorname{rank} 2)+2 *(\# \operatorname{rank} 3)+1 *(\# \operatorname{rank} 4)}{16}
$$

Similarly a preference score was calculated based on taste rankings. As both sweet and savoury products were presented twice in all 12 rankings the ranks-based score is divided by 24 and this preference score can range from 1.5 to 3.5 :

\section{relative preference score sweet}

$$
=\frac{4 *(\# \operatorname{rank} 1)+3 *(\# \operatorname{rank} 2)+2 *(\# \operatorname{rank} 3)+1 *(\# \operatorname{rank} 4)}{24}
$$

In case of no apparent preference for a specific category, all macronutrients would be ranked on each position twice and preference scores are 2.5.

As the preference scores for sweet and savoury are each other's opposites, i.e., savoury score = 5 - sweet score, we report only sweet preference scores in this article.

Data are presented as means with standard deviation (SD) unless otherwise specified. Data was analysed using IBM SPSS 21 (IBM Corporation, Armonk, New York, USA). Results were considered statistically significant at $\mathrm{p}<0.05$.

To compare liking of both test meals a paired-samples T-test was conducted. Repeated measures ANOVA was used to analyse appetite ratings and the preference scores with hunger state (before and after a meal) and taste of the test meal (sweet and savoury) as factors.

Non-parametric tests were used to determine relative preference for the different macronutrient and taste categories. To compare preference scores for macronutrient categories within conditions, e.g., before the sweet meal, Friedman ANOVA was used. Post-hoc analyses were done using Wilcoxon signed rank tests with Bonferroni correction. To determine relative preference for taste, sweet and savoury preference scores were compared using Wilcoxon signed rank tests. Pearson's correlations were determined to assess reproducibility of food preferences measured with the MTPRT. 
Liking scores were calculated by using the average liking rating within a category. These were analysed with repeated measures ANOVA to compare liking scores before and after the two different test meals. Post-hoc analyses were done using Bonferroni correction.

\section{Results}

\section{Liking of test meals}

Average liking of the sweet test meal was 40.7 ( $\mathrm{SD}=27.7)$, compared to 45.3 ( $\mathrm{SD}=23.2)$ for the savoury meal. Liking of both meals did not differ significantly, $\mathrm{T}(1,68)=1.12, p=0.27$.

\section{Appetite ratings}

Regardless of taste, eating a test meal decreased hunger, prospective consumption, appetite for something sweet and appetite for something savoury, but increased fullness (all $p<0.001$, Table 2.2). The interaction between hunger state and taste of the test meal showed that appetite for something sweet decreased more after eating the sweet test meal than after eating the savoury test meal, $F(1,68)=27.92, p<0.001$. Similarly, appetite for something savoury decreased more after eating the savoury test meal than after eating the sweet test meal, $F(1,68)=34.31, p<0.001$.

Table 2.2. Pre and post meal appetite ratings per type of meal

\begin{tabular}{lllll}
\hline & \multicolumn{2}{l}{ Sweet meal } & \multicolumn{2}{l}{ Savoury meal } \\
\cline { 2 - 5 } & Pre & Post $^{*}$ & Pre & Post* $^{*}$ \\
\hline Hunger & $71 \pm 16$ & $19 \pm 19$ & $67 \pm 15$ & $20 \pm 18$ \\
Fullness & $22 \pm 13$ & $72 \pm 20$ & $28 \pm 17$ & $76 \pm 18$ \\
Prospective consumption $^{*}$ & $66 \pm 14$ & $27 \pm 20$ & $64 \pm 15$ & $25 \pm 19$ \\
Appetite for sweet $^{1}$ & $58 \pm 23$ & $21 \pm 26$ & $55 \pm 23$ & $44 \pm 28$ \\
Appetite for savoury $^{1}$ & $73 \pm 15$ & $48 \pm 24$ & $68 \pm 18$ & $23 \pm 22$ \\
\hline
\end{tabular}

Ratings performed on a 100 point VAS. Values are means \pm SD. ${ }^{*}$ Post meal ratings are significantly different

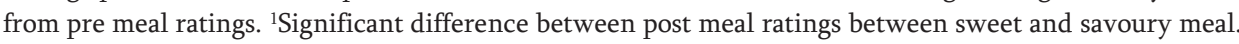

\section{Test-retest reliability of preference scores}

The preference scores in hungry state on the two test days did not significantly differ from each other (all $p>0.05$ ). In addition, these preference scores significantly correlated with each other within all macronutrient categories and the sweet category, $\mathrm{r}=0.77,0.68,0.78,0.69,0.74$ for highcarbohydrate, high-fat, high-protein, low-energy, and sweet respectively, all $p<0.001$ (Fig. 2.2). 


\section{A. Sweet}

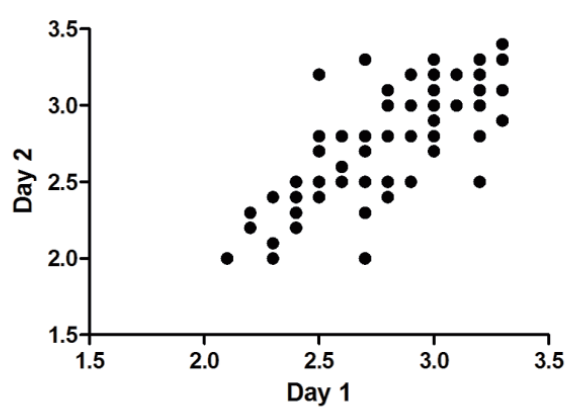

B. Carbohydrate

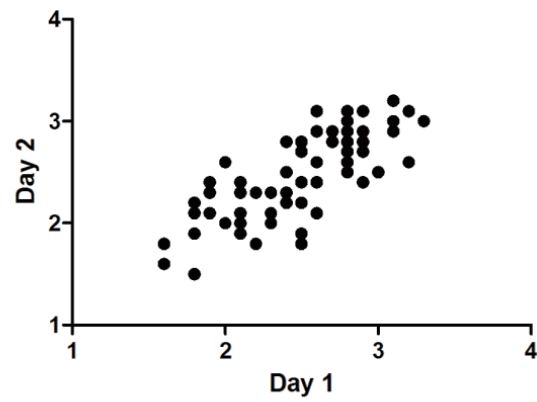

D. Protein

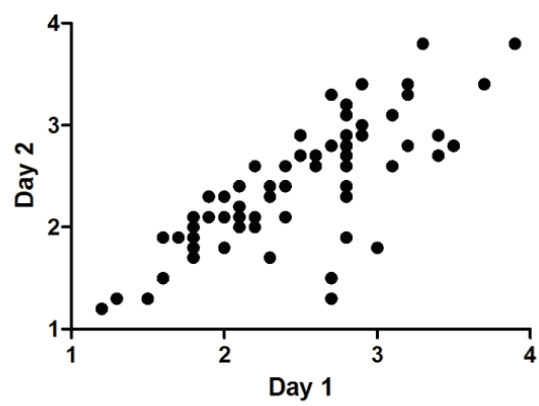

C. Fat

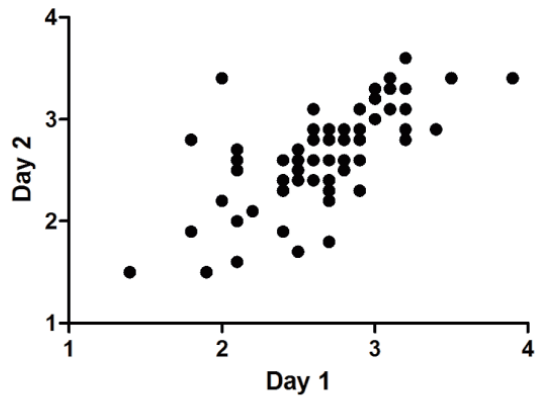

E. Low-energy

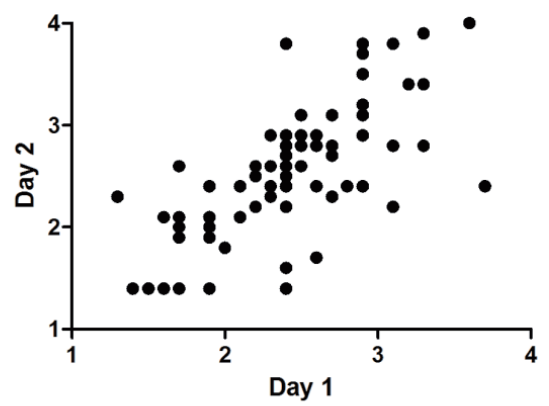

Figure 2.2. Correlation preference scores for sweet (A), high-carbohydrate (B), high-fat (C), highprotein (D) and low-energy (E) products in hungry condition between test day 1 and test day 2 .

\section{Food preferences}

Before consumption, preference scores for all categories did not differ between the sweet and savoury test meal conditions (all $p>0.05$ ). In both conditions, participants showed a relative preference for sweet over savoury (both $p<0.001$ ), but no relative preference for one of the macronutrient categories (Fig. 2.3). 
A. Carbohydrate

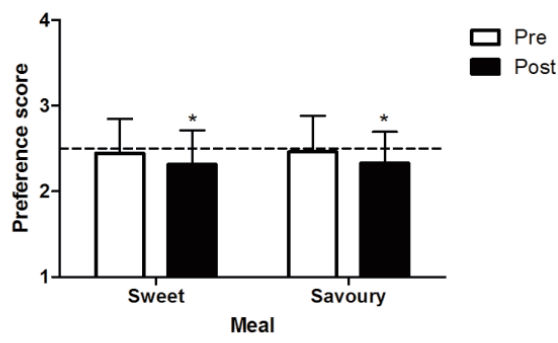

C. Protein

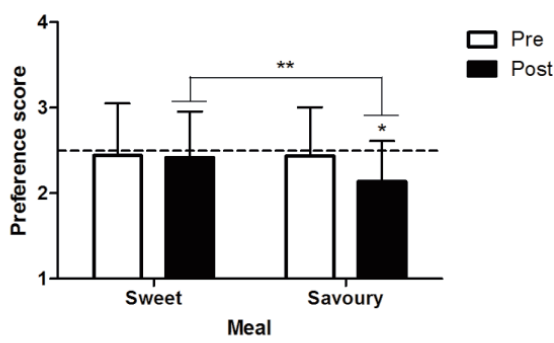

B. Fat

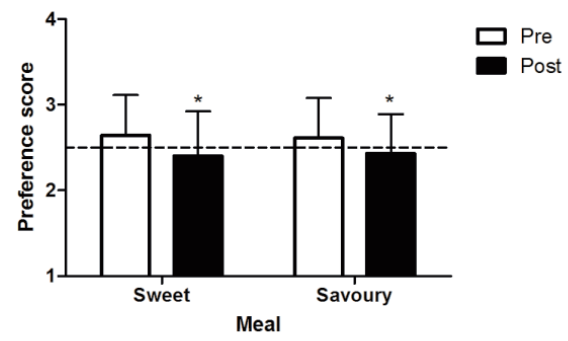

Figure 2.3. Preferences for high-carbohydrate (A), high-fat (B), high-protein (C) and low-energy (D) products $(m e a n \pm S D)$. Dotted line represents chance level. ${ }^{*}$ indicates significant change compared to pre meal at $p<0.05$ level, ${ }^{* *}$ indicates significant difference at $p<0.05$ level.

After consumption of the test meal, sweet preference shifted dependent on taste of the meal as shown by the interaction between hunger state and taste of the test meal: $F(1,68)=53.26, p<0.001$, partial $\eta^{2}=0.44$. Sweet preference decreased after the sweet meal and increased after the savoury meal (Fig. 2.4). After consumption of the sweet test meal, participants lost their relative preference for sweet products over savoury products, $\mathrm{Z}=-1.53, p=0.127$.

Fig. 2.3 shows the preferences for the four macronutrient categories. Preference for highcarbohydrate and for high-fat products decreased after meal consumption, regardless of taste, main effect carbohydrate: $\mathrm{F}(1,68)=18.87, p<0.001$, partial $\eta^{2}=0.22$; fat: $\mathrm{F}(1,68)=38.92, p<0.001$, partial $\eta^{2}=0.36$. Hunger state interacted with taste of the test meal for high protein foods, $F(1,68)=22.13$, $p<0.001$, partial $\eta^{2}=0.25$. This was explained by a decrease for high protein products after the savoury meal, but no change in preference for high protein products after the sweet meal. Preference for low-energy products increased after eating a meal, main effect: $\mathrm{F}(1,68)=85,30, p<0.001$, partial $\eta^{2}=0.56$. Hunger state interacted with taste of the test meal; low-energy preference increased more after the savoury meal than after the sweet meal $F(1,68)=22.13, p<0.001$, partial $\eta^{2}=0.11$.

After both meals, participants showed a relative preference for low-energy products compared to the other macronutrient categories (all $p<0.01$ ). After the savoury meal, high-fat products were preferred over high-protein products $(p<0.05)$. 


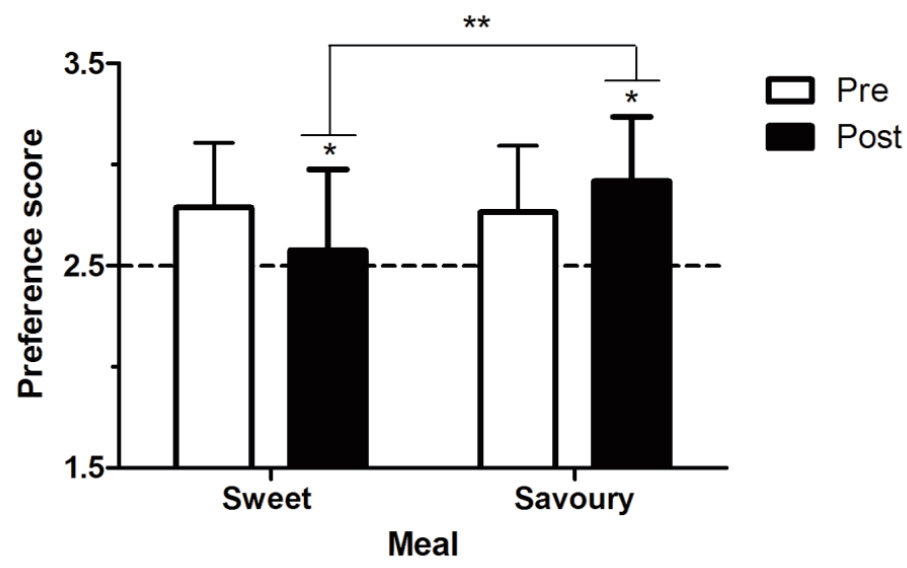

Figure 2.4. Preferences for sweet products before and after the sweet and savoury meal (mean $\pm S D$ ). Dotted line represents chance level. * indicates significant change compared to pre meal at $p<0.05$ level, ${ }^{* *}$ indicates significant difference at $p<0.05$ level.

\section{Liking of food categories}

Fig. 2.5 shows the liking ratings for the different food categories. After eating both test meals, liking of the sweet, savoury, high-carbohydrate, high-fat and high-protein products decreased (all $p<0.05)$, but liking of the low-energy products did not change $(p=0.832)$. For savoury, $\mathrm{F}(1,68)=10.96, p=0.001$, and for high-protein, $\mathrm{F}(1.68)=8.49, p=0.005$, hunger state interacted with taste of meal. For both categories, liking decreased more after the savoury meal than after the sweet meal. 
A. Sweet

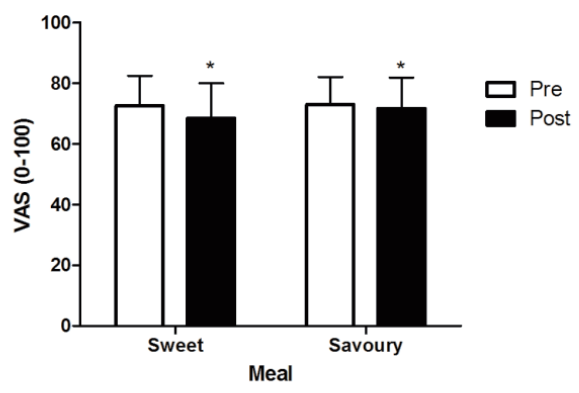

C. Carbohydrate

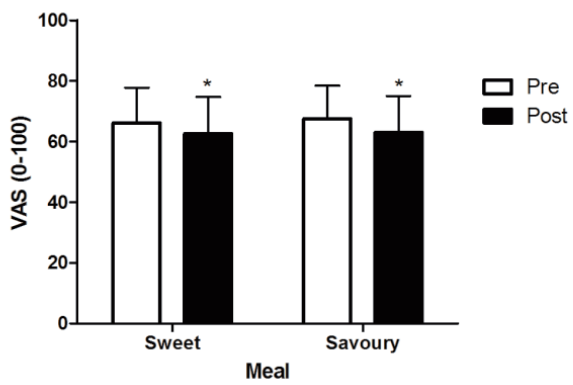

E. Protein

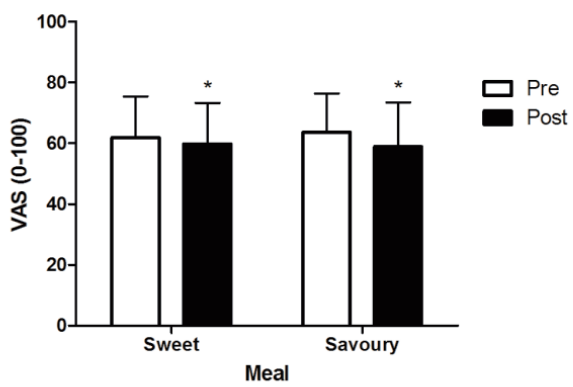

B. Savoury

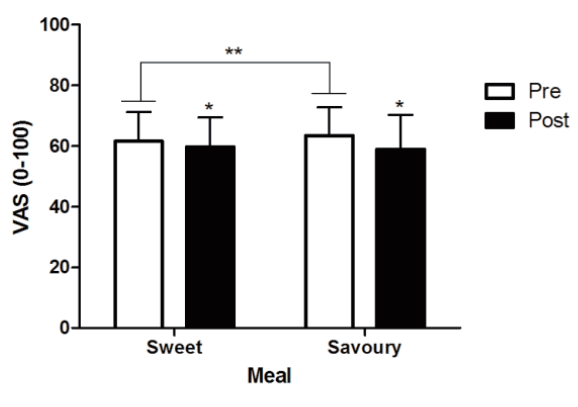

D. Fat

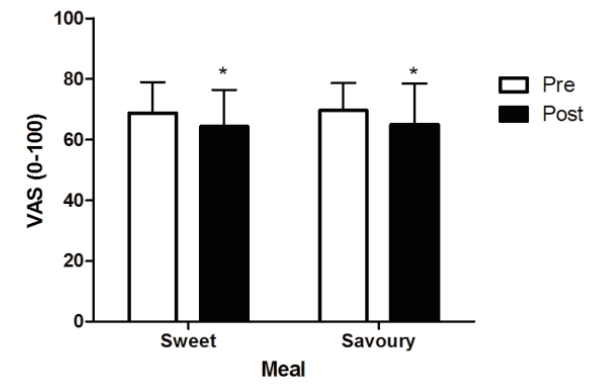

F. Lowenergy

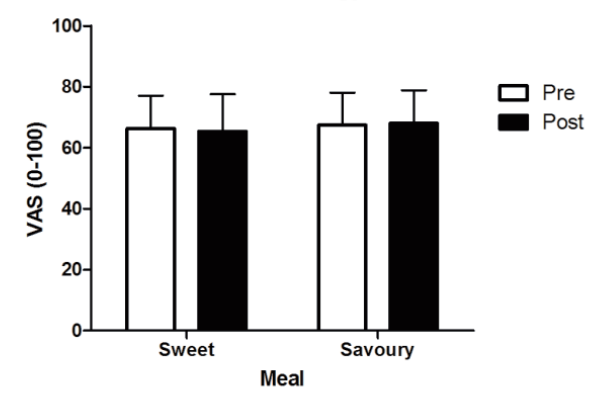

Figure 2.5. Liking ratings (0-100 VAS) for sweet (A), savoury (B), high-carbohydrate (C), high-fat (D), high-protein (E) and low-energy $(\mathrm{F})$ products $(\mathrm{mean} \pm \mathrm{SD}) .{ }^{*}$ indicates significant change compared to before meal at $p<0.05$ level. ${ }^{* *}$ indicates significant difference at $p<0.05$ level. 


\section{Discussion}

The current study demonstrates the reliability and validity of the Macronutrient and Taste Preference Ranking Task, the first food preference ranking task that includes both taste and four macronutrient dimensions. First, we showed that food preference scores for all categories in a hungry condition were highly correlated over two test sessions indicating test-retest reliability. Second, we showed a decreased preference for sweet products after eating a sweet meal and an increased preference for sweet products after eating a savoury meal. Preference for high-protein products decreased more after eating a savoury meal than it did after eating a sweet meal. Preferences for high-carbohydrate and high-fat products decreased after eating a meal, regardless of the taste of the meal. Preferences for low energy products increased after eating a meal, and this increase was most pronounced after the savoury meal. These results indicate that the MTPRT is a valid task to detect changes in food preferences.

Food preferences as measured with the MTPRT were shown to be reproducible. Correlations of preference scores for the different food categories as measured under similar circumstances were comparable to those found in previous studies that assessed test-retest reliability of methods to measure food preferences (Asao et al., 2012; Brisbois-Clarkson et al., 2009; Ledikwe et al., 2007). Given the reproducibility and the high variation in individual food preference scores between participants, the MTPRT may be able to identify individuals with specific preferences. Further studies are needed to assess whether there are specific individual characteristics that determine these individual food preferences.

In addition, the discriminative ability of the MTPRT was successfully demonstrated by assessing the effect of sensory-specific satiety. Similar to other methods used in previous studies, preferences for sweet decreased after the sweet meal and increased after the savoury meal (Griffioen-Roose et al., 2010; Guinard and Brun, 1998). Moreover, we were able to discriminate preferences between four different macronutrient categories, i.e., high-carbohydrate, high-fat, high-protein and low-energy. Preferences for high-carbohydrate and high-fat products decreased after eating a meal, regardless of the taste of the meal. Conversely, preference for low-energy products increased after meal consumption. This increased preference for low-energy products can be explained by the fact that participants were feeling full after the meal. This induced a shift from energy-dense to low-energy products and is consistent with other studies (Charbonnier et al., 2015a; Zoon et al., 2014). Preferences for high-protein products decreased after consumption of the savoury meal, but not after consumption of the sweet meal. This finding can be explained by the fact that savoury products are generally associated with high protein content (Lease et al., 2016; Viskaal Van Dongen et al., 2012). Contrarily, a previous study into the effects of taste of a 24-hour diet on food preferences did not find a difference in preference for protein (Griffioen-Roose et al., 2012a). However in that study, protein content of high-protein products was at most 26 energy percent and included both sweet and savoury products. This difference between high-protein products could explain these different findings. Another possible explanation could be that the preference for protein was confounded by preference for savoury. In that case, we would have expected an increased preference for protein after the sweet meal, similar to the increased preference for 
savoury. However, preference for protein did not change after the sweet meal. We therefore consider this explanation unlikely.

The meals in our study were carefully composed to be similar with regard to nutritional content, and only differed in the taste, sweet and savoury. Therefore we mainly observe effects which can be explained by the taste and detect less differentiation on the macronutrients. Most likely, when varying meals in macronutrient content pronounced changes in macronutrient preferences would become apparent (Barkeling et al., 1990; Hopkins et al., 2016; Ledikwe et al., 2007). Compared to the LFPQ the MTPRT has more macronutrient categories and can therefore be used in a wide range of studies that assess the influence of different factors on changes in preferences for different macronutrients and tastes in a variety of populations.

By using a ranking procedure, we can assess food preferences for categories of foods relative to other categories. In our study, we detected changes in preference for all food categories included in the MTPRT, while we did not detect these for all categories in the liking part. One important difference is that liking of sweet products decreased in a similar fashion after both the sweet and the savoury meal, whereas the preference score for sweet increased after the savoury meal. Furthermore, the ranking showed an increased preference for low-energy products after eating the meals, while liking of low-energy products did not change. Therefore, the ranking procedure is more sensitive compared to the liking procedure, and is thus able to detect more subtle shifts in food preferences.

The ranking method makes the MTPRT an easy and quick tool for evaluating food preferences over a wide range of food categories, i.e., high-carbohydrate, high-fat, high-protein and low-energy, both sweet and savoury. Because of this range, the food preference task can be used in a variety of studies without changing food categories based on the research question. In the current study the experiment was focused on assessing sensory-specific satiety in a controlled setting and within subjects. However food preferences are influenced by many factors and further research is needed to assess the use of the task in a less controlled, more natural setting, and to assess the effect of time of day, meal context and person characteristics like gender, age and eating type.

The current study did not measure actual food intake and thus cannot compare preference scores with actual intake. No conclusions can be drawn, therefore, on how food preferences as assessed with the MTPRT translate into actual behaviour. Based on previous comparisons of a forced-choice procedure with actual food intake (Griffioen-Roose et al., 2010; Griffioen-Roose et al., 2011), where strong correlations were found between relative food preference and food intake, we would hypothesize that the preference scores as measured with the MTPRT correlate with actual food choice behaviour.

It is important to note that the MTPRT does not include sweet high-protein products. Within the range of products that contain at least 50 energy percent of protein, there are no sweet products. The same applies to other food preference measures (Barkeling et al., 1990; BrisboisClarkson et al., 2009) and therefore we do believe that the products in the high-protein category are a good representation of this macronutrient. In addition, the food products currently included in the task are chosen based on their commercial availability in the Netherlands. As Dutch consumption patterns may differ from consumption patterns in other countries, it might be 
necessary to exchange a few individual food products in the MTPRT when using the task in other countries.

The MTPRT is the first food preference task that uses a ranking procedure and can detect shifts in preferences for both taste and macronutrients. The MTPRT was shown to be a reliable and valid method to measure food preferences, as results were reproducible under similar conditions, and differentiated food preferences based on macronutrients and taste in an experimental setting. The task can be used to examine the effect of internal and external factors that influence food preferences.

\section{Acknowledgements}

We would like to thank Lisette van Vliet, Erwin Ramai, Els Siebelink and Desiree Lucassen for their help in carrying out the study. 
Supplementary table 2.1. Nutritional values for the selected 32 products

\begin{tabular}{|c|c|c|c|c|c|c|}
\hline & & $\begin{array}{l}\text { Energy (kJ/100 } \\
\text { grams) }\end{array}$ & $\begin{array}{l}\text { Energy } \\
\text { (kcal/100 } \\
\text { grams) }\end{array}$ & $\begin{array}{l}\text { Protein } \\
(\mathrm{en} \%)\end{array}$ & $\begin{array}{l}\text { CHO } \\
\text { (en\%) }\end{array}$ & Fat (en\%) \\
\hline \multicolumn{7}{|c|}{ High carbohydrate } \\
\hline \multicolumn{7}{|c|}{ Sweet } \\
\hline & Ginger bread & 1346 & 318 & 3,4 & 88,9 & 5,9 \\
\hline & $\begin{array}{l}\text { Syrup waffles } \\
\text { (Dutch: Stroopwafels) }\end{array}$ & 1985 & 473 & 3,1 & 59,4 & 36,7 \\
\hline & Pancakes & 826 & 196 & 16,9 & 59,6 & 22,5 \\
\hline & Winegums & 1481 & 349 & 6,3 & 92,8 & 0,8 \\
\hline \multicolumn{7}{|c|}{ Savoury } \\
\hline & Tortilla chips & 2039 & 487 & 5,7 & 51,3 & 41,2 \\
\hline & French fries & 1282 & 306 & 5,9 & 50,1 & 42,1 \\
\hline & Nibbits & 1989 & 475 & 3,4 & 53,9 & 41,7 \\
\hline & Salty sticks & 1654 & 395 & 9,4 & 81,0 & 8,2 \\
\hline \multirow{2}{*}{\multicolumn{7}{|c|}{$\begin{array}{l}\text { High fat } \\
\text { Sweet }\end{array}$}} \\
\hline & & & & & & \\
\hline & $\begin{array}{l}\text { Chocolate bar } \\
\text { (milk chocolate) }\end{array}$ & 2278 & 546 & 5,1 & 40,2 & 53,6 \\
\hline & Cream pie & 1465 & 350 & 4,0 & 33,1 & 64,3 \\
\hline & $\begin{array}{l}\text { Apple turnover } \\
\text { (Dutch: Appelflap) }\end{array}$ & 1506 & 361 & 3,9 & 39,3 & 55,8 \\
\hline & $\begin{array}{l}\text { Large chocolate eclair (Dutch: } \\
\text { Bossche bol) }\end{array}$ & 1275 & 307 & 5,9 & 23,6 & 70,1 \\
\hline \multicolumn{7}{|c|}{ Savoury } \\
\hline & Salted peanuts & 2390 & 577 & 16,6 & 7,6 & 73,3 \\
\hline & Sausage roll & 1596 & 382 & 12,4 & 35,8 & 51,4 \\
\hline & Chips (salted) & 2254 & 541 & 4,7 & 37,9 & 55,7 \\
\hline & Cheese cubes & 1508 & 363 & 25,0 & 0,0 & 73,4 \\
\hline \multicolumn{7}{|c|}{ High protein } \\
\hline & Chicken satay & 667 & 158 & 78,2 & 0,0 & 21,6 \\
\hline & Cod fillet & 498 & 118 & 72,9 & 2,0 & 25,2 \\
\hline & Pork chop & 743 & 177 & 64,0 & 0,0 & 35,6 \\
\hline & Turkey fillet (cold cut) & 479 & 113 & 69,7 & 11,0 & 19,1 \\
\hline & Roast beef & 656 & 156 & 69,7 & 0,5 & 29,4 \\
\hline & Gammon (cold cut) & 569 & 136 & 53,5 & 7,1 & 38,4 \\
\hline & Shrimps & 397 & 94 & 84,3 & 0,4 & 15,3 \\
\hline & Steak & 616 & 146 & 80,3 & 0,0 & 19,7 \\
\hline \multicolumn{7}{|c|}{ Low energy } \\
\hline \multicolumn{7}{|c|}{ Sweet } \\
\hline & Peach & 172 & 41 & 9,8 & 77,1 & 0,0 \\
\hline & Strawberries & 123 & 29 & 9,7 & 70,3 & 0,0 \\
\hline & Apple & 254 & 60 & 1,3 & 86,7 & 3,0 \\
\hline & Melon galia & 107 & 25 & 0,0 & 96,0 & 0,0 \\
\hline \multicolumn{7}{|c|}{ Savoury } \\
\hline & Tomato & 96 & 23 & 12,2 & 53,9 & 19,6 \\
\hline & Cucumber & 55 & 13 & 18,5 & 58,5 & 13,8 \\
\hline & Pickles & 44 & 11 & 36,4 & 36,4 & 0,0 \\
\hline & Celery sticks & 60 & 14 & 28,6 & 57,1 & 0,0 \\
\hline
\end{tabular}




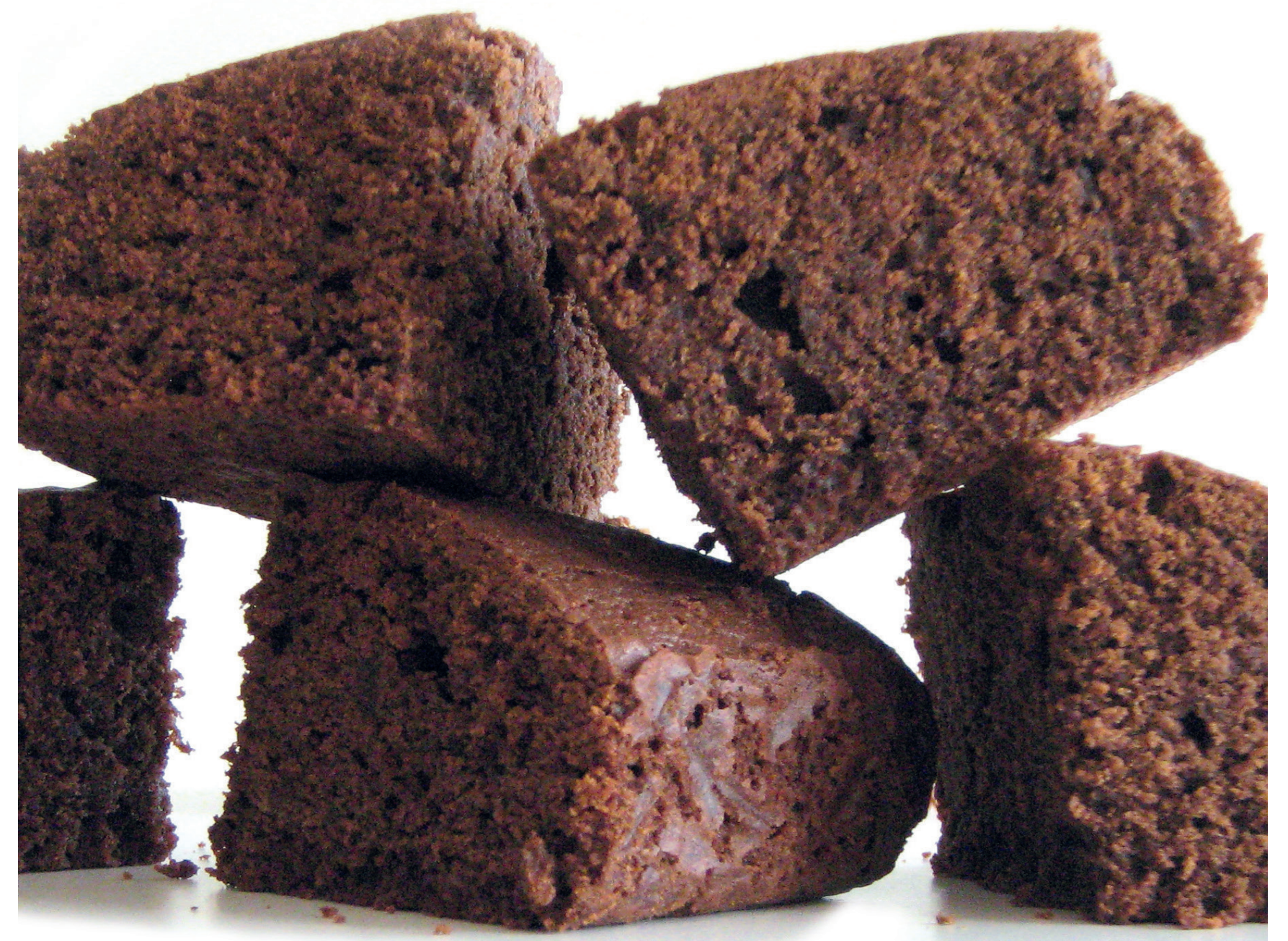




\section{CHAPTER 3}

EXPLORATIVE PLACEBO-CONTROLLED DOUBLE-BLIND INTERVENTION STUDY WITH LOW DOSES OF INHALED THC AND CBD REVEALS NO EFFECT ON SWEET TASTE INTENSITY PERCEPTION AND LIKING IN HUMANS

Suzanne E.M. de Bruijn

Cees de Graaf

Renger F. Witkamp

Gerry Jager

Published in Cannabis and Cannabinoid Research, Volume 2, Pages 114-122. 


\begin{abstract}
INTRODUCTION: The endocannabinoid system (ECS) plays an important role in food reward. For example, in humans liking of palatable foods is assumed to be modulated by endocannabinoid activity. Studies in rodents suggest that the ECS also plays a role in sweet taste intensity perception, but it is unknown to what extent this can be extrapolated to humans. Therefore, this study aimed at elucidating whether $\Delta 9$-tetrahydrocannabinol (THC) or cannabidiol (CBD) affect sweet taste intensity perception and liking in humans, potentially resulting in alterations in food preferences. MATERIALS AND METHODS: In a randomized placebo-controlled, double-blind, crossover study, ten healthy males participated in three test sessions that were two weeks apart. During the test sessions, participants received THC-rich, CBD-rich or placebo cannabis by inhalation divided over two doses (4 + $1 \mathrm{mg}$ THC; $25+10 \mathrm{mg}$ CBD). Participants tasted seven chocolate milk-like drinks that differed in sugar concentration and they rated sweet taste intensity and liking of the drinks. They were then asked to rank the seven drinks according to how much they liked the drinks and were offered ad libitum access to their favourite drink. In addition, they completed a computerized food preference task and completed an appetite questionnaire at the start, midway and at the end of the test sessions.

RESULTS: Inhalation of the cannabis preparations did not affect sweet taste intensity perception and liking, ranking order, or ad libitum consumption of the favourite drink. In addition, food preferences were not influenced by the interventions. Reported fullness was lower, whereas desire to eat was higher throughout the THC compared to the CBD condition.

CONCLUSIONS: These results suggest that administration of cannabis preparations at the low doses tested does not affect sweet taste intensity perception and liking, nor does it influence food preferences in humans.
\end{abstract}




\section{Introduction}

There is growing evidence that the endocannabinoid system (ECS) plays an important role in the regulation of hedonic hunger, i.e., consuming foods for the experience of reward and not for the compensation of any energy deficit (Lowe and Butryn, 2007). More specifically, the ECS seems to modulate palatability-dependent appetite (Argueta and DiPatrizio, 2017; DiPatrizio et al., 2011; DiPatrizio and Simansky, 2008; Droste et al., 2010), though the underlying mechanisms are far from clear.

It is important to note that palatability, or pleasantness, is not a fixed function of the sensory properties (e.g. taste, smell, texture) of a food, but also depends on the hedonic evaluation of more qualitative (liking) and contextual (eating occasion) characteristics (Cooper, 2004; Rogers, 1990).

Considering taste, there seem to be two ways in which taste intensity perception and liking can be modulated. One is via alterations in quantitative taste intensity perception, reflected by a shift in the psychophysical (concentration-intensity) function. In case of, for example, sweet taste, the same sugar concentration is perceived as more or less intensely sweet. The other way is related to changes in hedonic evaluation, reflected by a shift in the psychohedonic (intensity-pleasantness) function, where for example, the perceived sweetness intensity of a sugar concentration is unchanged, but the perceived sweetness is liked more or less (de Graaf et al., 1996).

In rodents, endocannabinoid activity modulates sweet taste palatability. Endocannabinoid stimulation enhances liking of sweet taste (De Luca et al., 2012; O'Brien et al., 2013) and motivational behaviour aimed at obtaining a sucrose solution (Higgs et al., 2003), whereas endocannabinoid inhibition reduces liking and motivational behaviour (Higgs et al., 2003; O'Brien et al., 2013). Some studies suggest that administration of CBD decreases food intake (Farrimond et al., 2012; Wierucka-Rybak et al., 2014), possibly via indirect antagonism of the ECS (McPartland et al., 2015; Pertwee, 2008), whereas other studies do not find an effect of CBD on food intake (Riedel et al., 2009; Wiley et al., 2005). Taken together, it is clear that CBD is far from being a classical ligand for the $\mathrm{CB}_{1}$ or $\mathrm{CB}_{2}$ receptor. The molecule displays a wide spectrum of activities on various molecular targets and produces multiple pharmacological effects (Ligresti et al., 2016; McPartland et al., 2015).

Administration of endocannabinoids was found to specifically increase responses to sweet taste in the Chorda tympani, the nerve that innervates the tongue, in wild-type mice, but not in $\mathrm{CB}_{1}$ knock-out mice (Yoshida et al., 2010). Administration of a $\mathrm{CB}_{1}$ antagonist decreased responses to sweet taste in the Chorda tympani (Niki et al., 2015). Together, these results suggest that modulating the ECS selectively changes the psychophysical function for sweet taste in mice.

In humans, anecdotal evidence suggests that the use of Cannabis preparations increases food palatability and intake (Abel, 1975; Foltin et al., 1988; Hart et al., 2002; Smit and Crespo, 2001; Tart, 1970), a phenomenon colloquially known as 'the munchies'. Furthermore, the $\mathrm{CB}_{1}$ inverse agonist rimonabant decreases food intake (Pi-Sunyer et al., 2006; Van Gaal et al., 2005). Thus far, only one study examined the effects of oral administration of delta-9-tetrahydrocannabinol (THC) on taste intensity and liking in humans. In a double-blind, placebo-controlled study, acute Cannabis administration was found not to affect taste intensity perception and liking for sweet, sour, salty and bitter food stimuli at baseline, and 2, 4 and 6 hours post-dosing. However, THC bioavailability 
varied widely and THC could not be detected in plasma of all participants (Mattes et al., 1994b), Based on the limited number of studies performed in humans, the aim of the current study was to further investigate whether modulating the ECS by administering phytocannabinoid preparations affects sweet taste intensity perception and liking in humans, and whether this results in altered food preferences.

\section{Methods}

\section{Participants}

Ten healthy, normal weight $\left(\mathrm{BMI}=21.7 \pm 1.2 \mathrm{~kg} / \mathrm{m}^{2}\right)$ males aged $23.4 \pm 1$ years participated in the current study. Participants were incidental cannabis-users to ensure familiarity with the psychotropic effects of cannabis use. Frequency of use ranged from four times a year to once a week. To limit the risk of adverse events, males were excluded from participation when they had ever experienced negative effects from using cannabis (e.g. bad trip, cannabis-induced psychosis). Other exclusion criteria were restraint eating (DEBQ: score >2.9; Van Strien et al., 1986), lack of appetite, difficulties swallowing or eating, having taste or smell disorders (self-report), following an energy restricted diet two months prior to the study, weight change $>5 \mathrm{~kg}$ during the two months prior to the study, being allergic or intolerant for the products under study. Participants were instructed to abstain from cannabis from two weeks before the first test session until study completion. Compliance was tested by means of a urine sample (SureScreen Diagnostics Ltd.) at the beginning of each test session. Participants were also asked not to use alcohol in the two days before a test session, and not to eat or drink anything except water or weak tea during the two hours before a test session. Participants gave written informed consent before the start of the study and they received financial compensation at the end of the study. The study was approved by the Medical Ethical Committee of Wageningen University and was conducted in accordance with the Helsinki declaration of 1975, as revised in 2008. The study was registered on clinicaltrials.gov as NCT02112292.

\section{Design and procedures}

The study had a randomized, placebo-controlled, double-blind, crossover design. All participants completed one practice session (familiarization with the test procedures) and three test sessions after administration of cannabis either rich in THC, CBD or placebo (for details see "Study drugs and administration"). Test sessions were two weeks apart to allow for complete clearance of drugs.

Each test session consisted of repeated measurements of appetite and subjective effects using visual analogue scales (VAS), venous blood sampling to determine plasma concentrations of THC and CBD, a taste test with chocolate milk-like drinks that differed in sugar concentration (for details see "Sweet taste intensity perception and liking"), a control task, a ranking test with the drinks, ad libitum intake of the preferred chocolate milk-like drink, and the Macronutrient and Taste Preference Ranking Task (MTPRT; (de Bruijn et al., 2017). See Figure 3.1 for a schematic overview of the test procedure. 


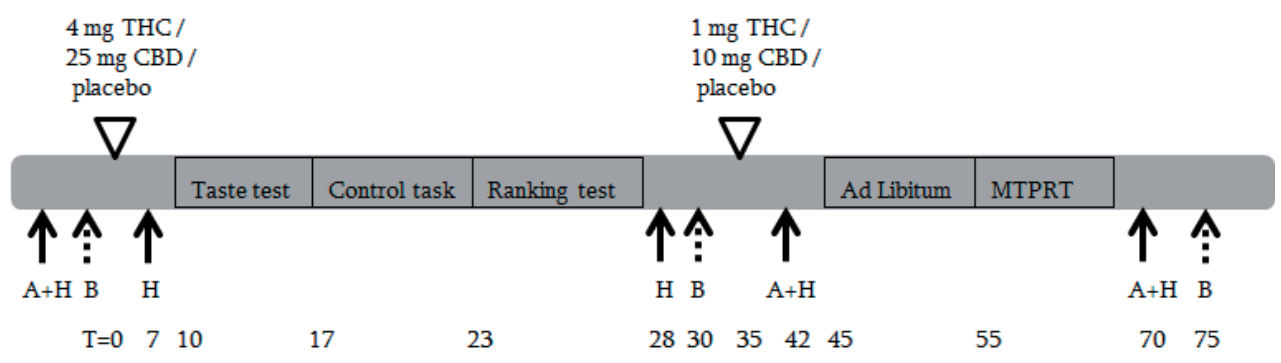

Figure 3.1. Schematic overview of a test session. $\mathrm{A}=$ appetite questionnaire, $\mathrm{H}=$ feeling high (VAS), $\mathrm{B}=$ blood sample, MTPRT $=$ Macronutrient and Taste Preference Ranking Task, $\mathrm{T}=$ time

\section{Study drugs and administration}

The active preparations consisted of the dried, milled and homogenized flowers of two varieties of the plant $C$. sativa and were obtained from the Office for Medicinal Cannabis (OMC; The Netherlands); variety Bedrocan ${ }^{\circledR}\left(19 \%\right.$ THC, $<1 \%$ CBD), and variety Bedrolite ${ }^{\circledR}(<1 \%$ THC, $9 \% \mathrm{CBD}$ ). The placebo (variety "Bedrocan" ${ }^{\circledR} ;<0.5 \% \mathrm{THC}$ ) had a moisture content and terpenoid profile (providing the typical smell and taste of cannabis) identical to the active drug, and was obtained from Bedrocan BV (official supplier to the OMC; Kowal et al., 2015). From here onwards we will refer to the three treatments as the THC (Bedrocan $\left.{ }^{\circledR}\right)$, CBD (Bedrolite ${ }^{\circledR}$ ) and placebo condition. In the THC condition, participants received an initial dose of four $\mathrm{mg}$ of THC, followed by one mg of THC as a top-up dose after 35 minutes to maintain stable levels of central nervous system effects (Kleinloog et al., 2014). In the CBD condition, the initial and top-up dose of CBD were 25 and $10 \mathrm{mg}$, respectively.

For each individual dose, exact amounts of Bedrocan ${ }^{\circledR}$ or Bedrolite ${ }^{\circledR}$ and placebo were mixed so that each dose was equal to $250 \mathrm{mg}$ of plant preparation total weight. According to GMP guidelines and to ensure double-blindness, study medication was prepared, packed and coded by ACE Pharmaceuticals BV (Zeewolde, The Netherlands), a company licenced to prepare trial medication. Study medication was stored at a temperature of $-18^{\circ} \mathrm{C}$ and taken out of the freezer thirty minutes before the start of a test session. Administration was performed with a MINIVAP vaporizer (HERMES MEDICAL ENGINEERING, S.L) that was set at a temperature of $230^{\circ} \mathrm{C}$. For each administration, participants were instructed to inhale 12 times with a duration of five seconds per inhalation and a pause of 25 seconds between adjacent inhalations.

\section{Plasma levels and subjective effects}

Venous blood samples were collected in $\mathrm{NaF} / \mathrm{KOx}$ (anticoagulant) tubes at baseline ( $\mathrm{T}=0$ ), $\mathrm{T}=30$ and $\mathrm{T}=75$ minutes, and stored at $4^{\circ} \mathrm{C}$ until the end of the test session. Then, tubes were centrifuged at $1300 \mathrm{~g}$ for 10 minutes at $4^{\circ} \mathrm{C}$ and plasma samples were collected and stored at $-80^{\circ} \mathrm{C}$ until analyses. Plasma was analysed for THC and CBD concentrations (ng/ml) using LC-MS/MS by Analytical Biochemical Laboratory BV (Assen, The Netherlands). Due to budget restraints, the samples of the placebo condition were only analysed for half of the sample $(n=5)$.

Subjective effects of the treatments were assessed with a VAS on which participants rated 'feeling high' throughout the test sessions. 


\section{Sweet taste intensity perception and liking}

A series of chocolate milk-like drinks that differed in sugar concentration was used to assess sweet taste intensity perception and liking. Samples were prepared freshly on the morning of each test session. First, a basic drink was prepared, containing vanilla sugar (Ruf; 2\%), Nutilis powder (Nutricia;3\%), cream (De Zaanse Hoeve; 18\%), skimmed milk (De Zaanse Hoeve; 74.5\%), and cocoa powder (Blooker; 2.5\%). Then, different amounts of basic chocolate milk-like drink and sucrose (Van Gilse) were mixed to get seven drinks with 2, 4.4, 6.7, 10, 15, 22.5, 33.8 grams of added sugar per $100 \mathrm{ml}$, respectively (Table 3.1). These sugar concentrations were chosen to cover a full psychophysical sweetness intensity curve, ranging from not that sweet to very sweet.

The drinks were coded with random three-digit numbers and presented to the participants in $20 \mathrm{~mL}$ samples, in a random order that differed between participants, but was kept constant per participant across test sessions. Participants were instructed to take a sip and then first rate sweet taste liking, followed by sweet taste intensity on a 200 point labelled affective magnitude scale (Schutz and Cardello, 2001) and a 100 point labelled magnitude scale (Green et al., 1993) respectively. In between samples, participants were instructed to rinse their mouth with water and wait 30 seconds before tasting the next sample.

Table 3.1. Composition of the different chocolate milk-like drinks per $100 \mathrm{ml}$.

\begin{tabular}{|c|c|c|c|}
\hline $\begin{array}{l}\text { Added sugar } \\
(\mathrm{g} / 100 \mathrm{ml})\end{array}$ & Total weight (g) & Basic (g) & Sugar $(\mathrm{g})$ \\
\hline 2 & 85 & 85 & 0 \\
\hline 4.4 & 86 & 83.6 & 2.4 \\
\hline 6.7 & 87 & 82.3 & 4.7 \\
\hline 10 & 88.6 & 80.6 & 8 \\
\hline 15 & 91.5 & 78.5 & 13 \\
\hline 22.5 & 95.7 & 75.2 & 20.5 \\
\hline 33.8 & 102.2 & 70.4 & 31.8 \\
\hline
\end{tabular}

The basic drink contained $2 \mathrm{~g} / 100 \mathrm{ml}$ added vanilla sugar.

The drinks were coded with random three-digit numbers and presented to the participants in $20 \mathrm{~mL}$ samples, in a random order that differed between participants, but was kept constant per participant across test sessions. Participants were instructed to take a sip and then first rate sweet taste liking, followed by sweet taste intensity on a 200 point labelled affective magnitude scale (Schutz and Cardello, 2001) and a 100 point labelled magnitude scale (Green et al., 1993) respectively. In between samples, participants were instructed to rinse their mouth with water and wait 30 seconds before tasting the next sample.

\section{Control task}

To check whether the cannabis treatments did not affect task performance or perception in general, a control task was added to the procedures. Participants were asked to rate the greyness of seven shades of grey on a 100 point VAS that was anchored by 'white' and 'black' (de Graaf et al., 1996). Reflection percentages of the different shades were $96,84,64,44,20,8$ and $4 \%$ respectively, 
with shades ranging from almost white to almost black. Analyses showed that ratings did not differ between conditions and that participants were able to differentiate between the different shades of grey (results not reported).

\section{Appetite, ranking task and ad libitum intake}

Throughout the test sessions participants three times (see Fig 3.1) rated levels of hunger, fullness, desire to eat and prospective consumption on a 100 point VAS anchored by 'not at all' and 'extremely', except for prospective consumption which was anchored by 'nothing at all' and 'a very large amount' (Blundell et al., 2010).

After the control task, participants were presented with $80 \mathrm{~mL}$ of the seven chocolate milklike drinks and were asked to rank them according to how much they liked the drinks. During the ad libitum intake test, participants were presented with one litre of their favourite drink, which they could consume ad libitum for 10 minutes.

\section{Food preferences}

Food preferences were assessed using the MTPRT. In this task, participants were presented with four pictures of different food products at a time and asked to rank the products according to what they most desire to eat at that moment. Food products included in this task were either high in carbohydrate, high in fat, high in protein or low in energy, and had a sweet or savoury taste. Examples of foods included in the food preference test are pancakes (high-carbohydrate sweet), salty sticks (high-carbohydrate savoury), chocolate bar (high-fat sweet), cheese cubes (high-fat savoury), shrimps (high-protein), strawberries (low-energy sweet) and cucumber (low-energy savoury). In short, the task consists of a macronutrient section, in which participants are presented with one product from each of the four macronutrient categories, and a taste section, in which participants are presented with two sweet and two savoury products. Based on the rankings, preference scores for different macronutrient and taste categories, i.e. high-carbohydrate, high-fat, high-protein and low-energy, and sweet and savoury are calculated as described elsewhere (de Bruijn et al., 2017). Preference scores for the macronutrient categories can range from 1 to 4 , with a higher score indicating a higher preference for a category. A score of 1 indicates all products from that category were ranked last, a score of 4 indicates all products from that category were ranked first. As each ranking in the taste section comprises two sweet and two savoury products, preference scores for sweet and savoury can range from 1.5 to 3.5. Because preference scores for sweet and savoury are each other's opposite, we only report preference scores for sweet in this article.

\section{Data analyses}

Data are presented as means with standard error of the mean (SEM). Data was analysed using IBM SPSS 22 (IBM Corporation, Armonk, New York, USA). Results were considered statistically significant at $\mathrm{p}<0.05$. When statistically significant main effects were found, post-hoc tests were performed using Bonferroni correction.

Liking ratings of the chocolate milk-like drinks were transferred to 100 points by dividing the scores by two. Using repeated measures ANOVA, rated sweetness intensity and liking of the drinks were compared, with condition (THC, CBD and placebo) and sugar concentration as factors. 
For the ranking task, the mean rank was calculated for each chocolate milk-like drink in each condition. Friedman ANOVA was used to compare mean ranks between conditions for each drink separately and to compare ranks of the drinks within conditions. For the ad libitum task, the consumed amount and energy content of the consumed drink were used to calculate the consumed energy. Repeated measures ANOVA was used to test if the consumed energy differed between conditions.

Preference scores from the MTPRT were compared between conditions using repeated measures ANOVA. To compare preference scores within each condition, Friedman ANOVA was used for the macronutrient categories and Wilcoxon signed rank tests were used to compare the sweet and savoury preference scores.

Appetite ratings were compared using repeated measures ANOVA with condition (THC, $\mathrm{CBD}$, placebo) and time as factors. Because of one missing data point for one participant, mixed models ANOVA was used to analyse 'feeling high' with condition, time and condition"time as fixed factors and subject as a random factor.

\section{Results}

\section{Plasma levels and subjective effects}

Table 3.2 shows the mean TCH and CBD plasma levels for all three conditions. In some participants, low levels of CBD were detected in the THC condition and vice versa. Low levels of THC were detected in plasma samples after administration of placebo, but these were 8- vs. 6.5-fold lower as compared to $\mathrm{T}=30$ and $\mathrm{T}=75 \mathrm{~min}$ in the $\mathrm{THC}$ condition.

Table 3.2. THC and CBD plasma levels for all three conditions.

\begin{tabular}{llll}
\hline Condition & Compound & $\mathrm{T}=\mathbf{3 0}$ & $\mathrm{T}=\mathbf{7 5}$ \\
\hline THC & THC & $1.6 \pm 0.5$ & $1.3 \pm 0.4$ \\
& CBD & $0.34 \pm 0.1$ & $0.26 \pm 0.1$ \\
CBD & THC & 0.16 & $0.21 \pm 0.03$ \\
& CBD & $2.8 \pm 0.8$ & $3.0 \pm 0.7$ \\
Placebo & THC & $0.22 \pm 0.05$ & $0.21 \pm 0.04$ \\
& CBD & ND & ND \\
\hline
\end{tabular}

Plasma levels in $\mathrm{ng} / \mathrm{ml}$. Values are means \pm SEM. $\mathrm{ND}=$ not detected. $\mathrm{n}=10$ for THC in THC condition and CBD in CBD condition. CBD was detected in 4 participants ( $\mathrm{T}=30$ and $\mathrm{T}=75$ ) in the THC condition, THC was detected in $1(\mathrm{~T}=30)$ and in $3(\mathrm{~T}=75)$ participants in the CBD condition. Placebo condition was tested for 5 participants. THC was detected in $4(\mathrm{~T}=30)$ and in $5(\mathrm{~T}=75)$ participants.

As shown in Figure 3.2, reported levels of 'feeling high' differed between conditions, $\mathrm{F}(2,13)=4.60, \quad p<0.05$, and over time, $\mathrm{F}(4,22)=7.35, \quad p<0.05$. Post-hoc analyses showed that participants felt significantly more 'high' in the THC compared to the placebo condition. 


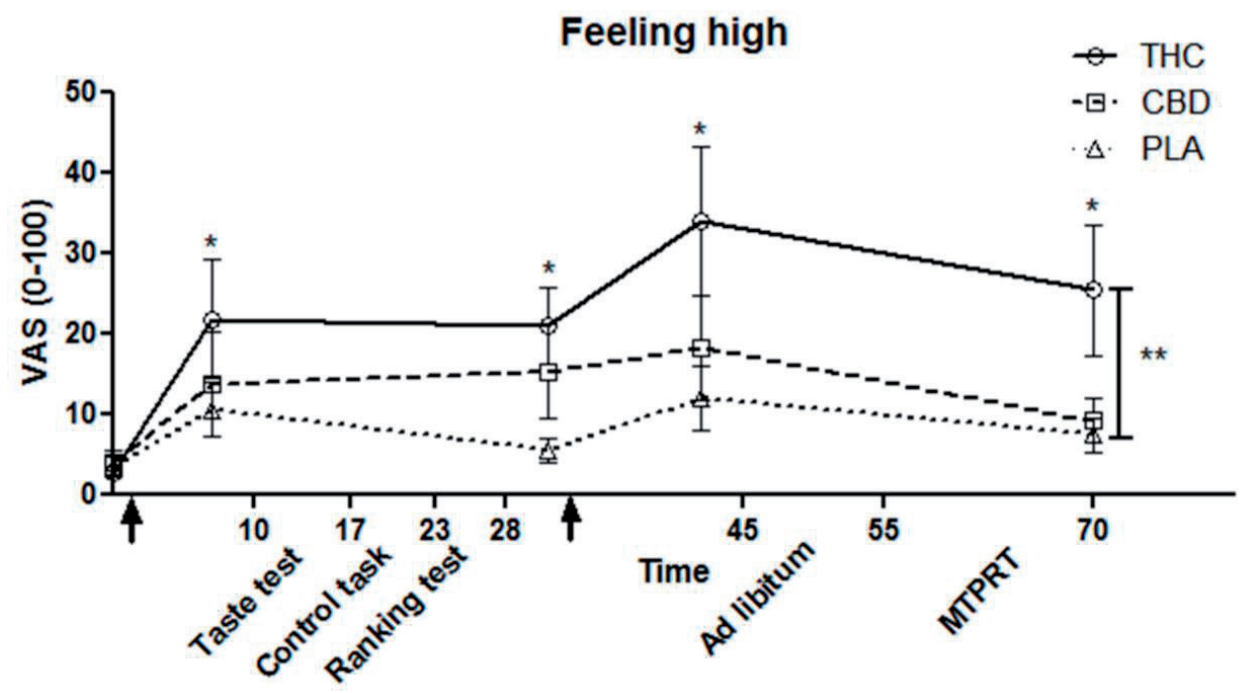

Figure 3.2. Reported 'feeling high' (means \pm SEM). Arrows indicate treatment administration. For the first arrow: $4 \mathrm{mg}$ THC, $25 \mathrm{mg}$ CBD or placebo. For the second arrow: $1 \mathrm{mg}$ THC, $10 \mathrm{mg}$ CBD or placebo. ${ }^{*}$ indicates significant difference from $\mathrm{T}=0(p<0.05) .{ }^{* *}$ indicates difference between THC and placebo conditions $(p<0.05)$. PLA=placebo.

\section{Sweet taste intensity perception and liking}

Drinks that contained more sugar were perceived as more sweet, $\mathrm{F}(6,54)=57.87, p<0.05$, but perceived sweet taste intensity of the seven chocolate milk-like drinks did not differ between the three conditions, $\mathrm{F}(2,18)=0.66, p=0.53$ (Fig 3.3A). Liking of the different drinks differed with sugar concentration, $\mathrm{F}(6,54)=2.47, p<0.05$, where the drinks that contained the least and the most sugar were liked least. Similar to the intensity findings, the liking of the seven drinks did not differ between conditions, $\mathrm{F}(2,18)=0.94, p=0.41$ (Fig 3.3B).

\section{Ranking task}

Ranks of the different drinks did not differ between conditions, all $p>0.05$. In addition, the ranking task did not show differences between the drinks, $\mathrm{X}^{2}(6)=11.10, p=0.085, \mathrm{X}^{2}(6)=12.73, p=0.048$, and $\mathrm{X}^{2}(6)=12.51, p=0.051$ for THC, CBD and placebo respectively.

\section{Appetite and ad libitum intake}

Results from the appetite questionnaire can be found in Figure 3.4. Reported hunger did not differ between conditions, $\mathrm{F}(2,18)=2.62, p=0.10$, and did not change over time, $\mathrm{F}(2,18)=2.47, p=0.11$. However, reported fullness differed between conditions, $\mathrm{F}(2,18)=3.74, p<0.05$, and changed over time, $\mathrm{F}(2,18)=4.38, p<0.05$. Throughout the whole test session, fullness ratings were higher in the $\mathrm{CBD}$ condition than in the THC condition. Desire to eat differed between conditions, $\mathrm{F}(2,18)=6.50$, $p<0.05$, and was higher in the THC condition than in the CBD condition throughout the whole test session, but did not change over time, $\mathrm{F}(2,18)=2.43, p=0.12$. Prospective consumption differed between conditions, $\mathrm{F}(2,18)=4.83, p<0.05$, but did not change over time, $\mathrm{F}(2,18)=3.34, p=0.06$. 

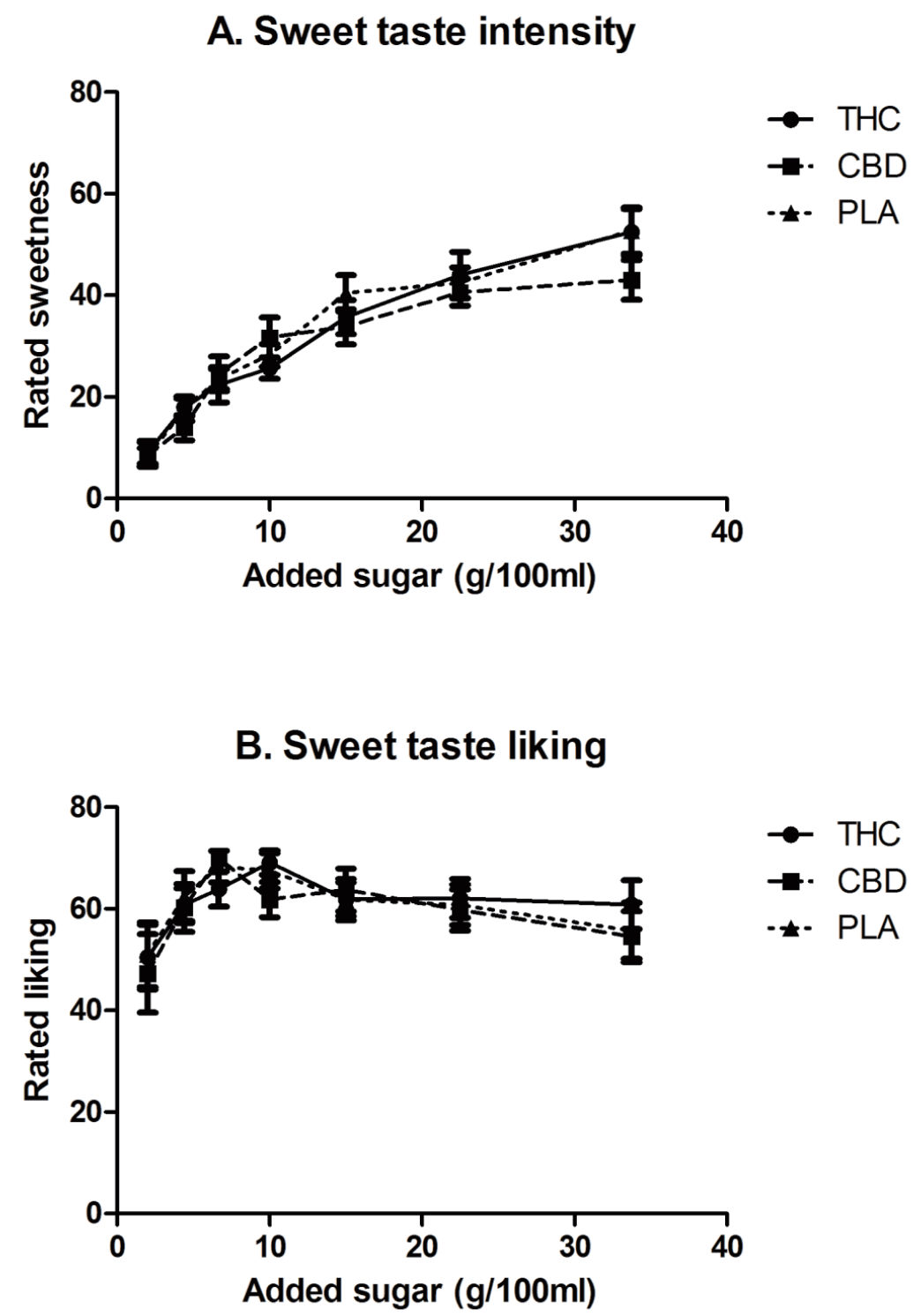

Figure 3.3. Ratings (means \pm SEM) for A) Sweet taste intensity, and B) Sweet taste liking. PLA = placebo. Drinks were rated as more intensely sweet with increasing sugar concentration. The drinks with the least and the most amount of added sugar were liked less $(p<0.05)$. 
A. Hunger

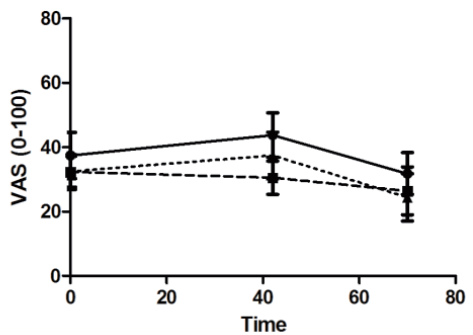

C. Desire to eat

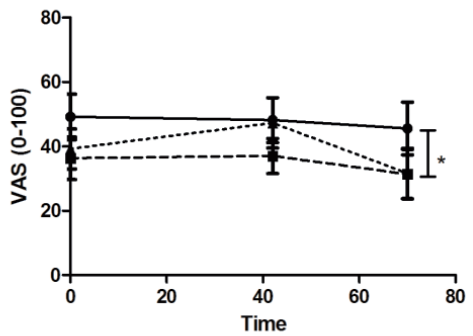

B. Fullness

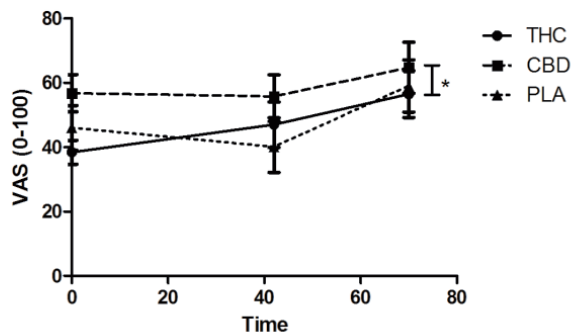

Figure 3.4. Rated scores (means \pm SEM) for A) hunger, B) fullness, C) desire to eat and D) prospective consumption. ${ }^{*}$ indicates difference throughout THC and CBD condition $(p<0.05)$. PLA = placebo.

Ad libitum intake of the most liked chocolate milk-like drink did not differ between conditions, $\mathrm{F}(2,18)=0.50, p=0.62$. Participants consumed on average $537 \pm 67 \mathrm{kcal}$ in the THC condition, $581 \pm 66 \mathrm{kcal}$ in the CBD condition and $595 \pm 65 \mathrm{kcal}$ in the placebo condition.

\section{Food preferences}

The preference scores can be found in Table 3.3. Treatments did not affect preference for sweet or for any of the macronutrients, all $p>0.05$. In all three conditions, participants had a preference for savoury over sweet, all $p<0.05$, but not for one of the macronutrients over the others, all $p>0.05$.

Table 3.3. Food preference scores in all three conditions.

\begin{tabular}{llll}
\hline & THC & CBD & Placebo \\
\hline High-carbohydrate & $2.39 \pm 0.07$ & $2.30 \pm 0.13$ & $2.43 \pm 0.14$ \\
High-fat & $2.61 \pm 0.15$ & $2.42 \pm 0.12$ & $2.42 \pm 0.11$ \\
High-protein & $2.74 \pm 0.26$ & $2.74 \pm 0.20$ & $2.84 \pm 0.19$ \\
Low-energy & $2.26 \pm 0.25$ & $2.54 \pm 0.26$ & $2.31 \pm 0.24$ \\
Sweet & $2.20 \pm 0.09$ & $2.22 \pm 0.08$ & $2.20 \pm 0.10$
\end{tabular}

Values are means \pm SEM. Preference scores for macronutrient categories can range between 1 and 4, preference score for sweet can range between 1.5 and 3.5. 


\section{Discussion}

To the best of our knowledge, the current study is the first randomized, placebo-controlled, double-blind study investigating sweet taste intensity perception, sweet taste liking and possible consequent changes in food preference following inhalation of THC and CBD. The results indicate no changes in sweet taste intensity perception and liking, nor in food preferences, as related to taste (sweet versus savoury) and macronutrient composition (carbohydrate, protein, fat, low-energy).

The lack of effects on sweet taste intensity and liking after activating or inhibiting the ECS is contradictory to our expectations based on previous studies that showed endocannabinoid modulation of sweet taste intensity perception in mice (Niki et al., 2015; Yoshida et al., 2010). However, the current findings are in line with a study in humans that assessed taste intensity perception after oral THC administration. In that study, THC did not affect intensity and liking of sweetness, saltiness, sourness and bitterness (Mattes et al., 1994b).

In line with the results from the taste test, preference for sweet taste in the MTPRT did not differ between the THC, CBD and placebo conditions. In addition, preference for macronutrients was not affected by administration of THC or CBD. Earlier studies in humans showed that cannabis increases intake of snack foods, with a specific increased intake of sweet snacks (Abel, 1975; Foltin et al., 1988).

In the current study, participants had a preference for savoury over sweet. Just before completing the MTPRT, participants had consumed a large amount of a sweet drink to satiety. This might have caused sensory-specific satiety, i.e. decreased pleasantness of sensory properties of the food that has just been consumed to satiety (Rolls et al., 1981). The effect of sensory-specific satiety might have overruled a potential effect of endocannabinoid modulation on preference for sweet taste. However, given that THC and CBD administration did not influence sweet taste intensity perception and liking of the chocolate milk-like drinks, we think it is more likely that there is no effect of endocannabinoid modulation on sweet taste preference.

Desire to eat was higher and fullness was lower in the THC condition compared to the CBD condition, possibly related to baseline differences. However, despite these differences, ad libitum intake of the most preferred chocolate milk-like drink did not differ between conditions. In a previous study in cancer patients, THC treatment increased appetite, but did not affect total energy intake (Brisbois et al., 2011). Also in a healthy population, THC did not affect total energy intake (Mattes et al., 1994a). However, other studies in humans showed that THC increases food intake (Abel, 1975; Foltin et al., 1988). CBD has previously been shown not to affect appetite in patients that suffer from type 2 diabetes (Jadoon et al., 2016). In rats, however, CBD has been shown to decrease food intake (Farrimond et al., 2012; Wierucka-Rybak et al., 2014), which may be caused by higher fullness. These contradicting findings warrant further investigation.

The plasma levels of THC and CBD showed that the administration of pharmacological doses by inhalation was successful in our study. The placebo cannabis contained traces of THC, which led to detectable but very low THC plasma levels in the placebo condition. As THC plasma levels in the placebo condition were eight times lower than in the THC condition, the functional consequences on outcome measures are likely minimal. 
The relatively small sample size could be regarded as a limitation of our exploratory study. However, we would expect to find similar results in a larger sample. With ten participants, we see a great overlap of the mean sweet taste intensity and liking of the different drinks, which suggests that we will also not see any differences with more participants included in the study. Especially for the sweet taste liking, there is a large variation between participants. For the food preference task, one may expect to find differences in preference for low-energy products with a larger sample size. As CBD increases fullness and decreases desire to eat, we would expect that it also increases preference for low-energy products based on previous experience with the MTPRT (de Bruijn et al., 2017).

The current study included only male participants. As endocannabinoid pharmacology differs between males and females (Craft et al., 2013; Diaz et al., 2009), females might respond differently to inhaled THC and CBD. Future studies should take this into account and consider to include both males and females.

Another plausible explanation for our negative results might be that we used a relatively low dose of THC compared to other studies (Kleinloog et al., 2014; Kowal et al., 2015). We chose this dose as we did not want obvious intoxication to influence the results. However, it is possible that the dose is too low to evoke an effect and that we would have seen an effect of THC on sweet taste intensity perception and liking if we would have used a higher dose. Importantly, the effects of THC and other $\mathrm{CB}_{1}$ agonists usually follow an inverted u-shape, i.e., there is an optimal dose at which the largest effect occurs and for higher doses, the effect wears off (Bellocchio et al., 2010; DiPatrizio and Simansky, 2008; Wiley et al., 2005). Dosing too high could therefore also lead to no effect. Future studies should take this in consideration.

Based on the results from the current study, we conclude that challenging the ECS with low doses of THC or CBD does not influence sweet taste intensity perception and liking in humans. In addition, low doses of THC and CBD do not affect food preferences.

\section{Acknowledgments}

We would like to thank Pauline Claessen, research dietician, for providing the recipe for the chocolate milk-like drinks, and Tim Crolla and Michele Pedrotti for their help in carrying out the study. 


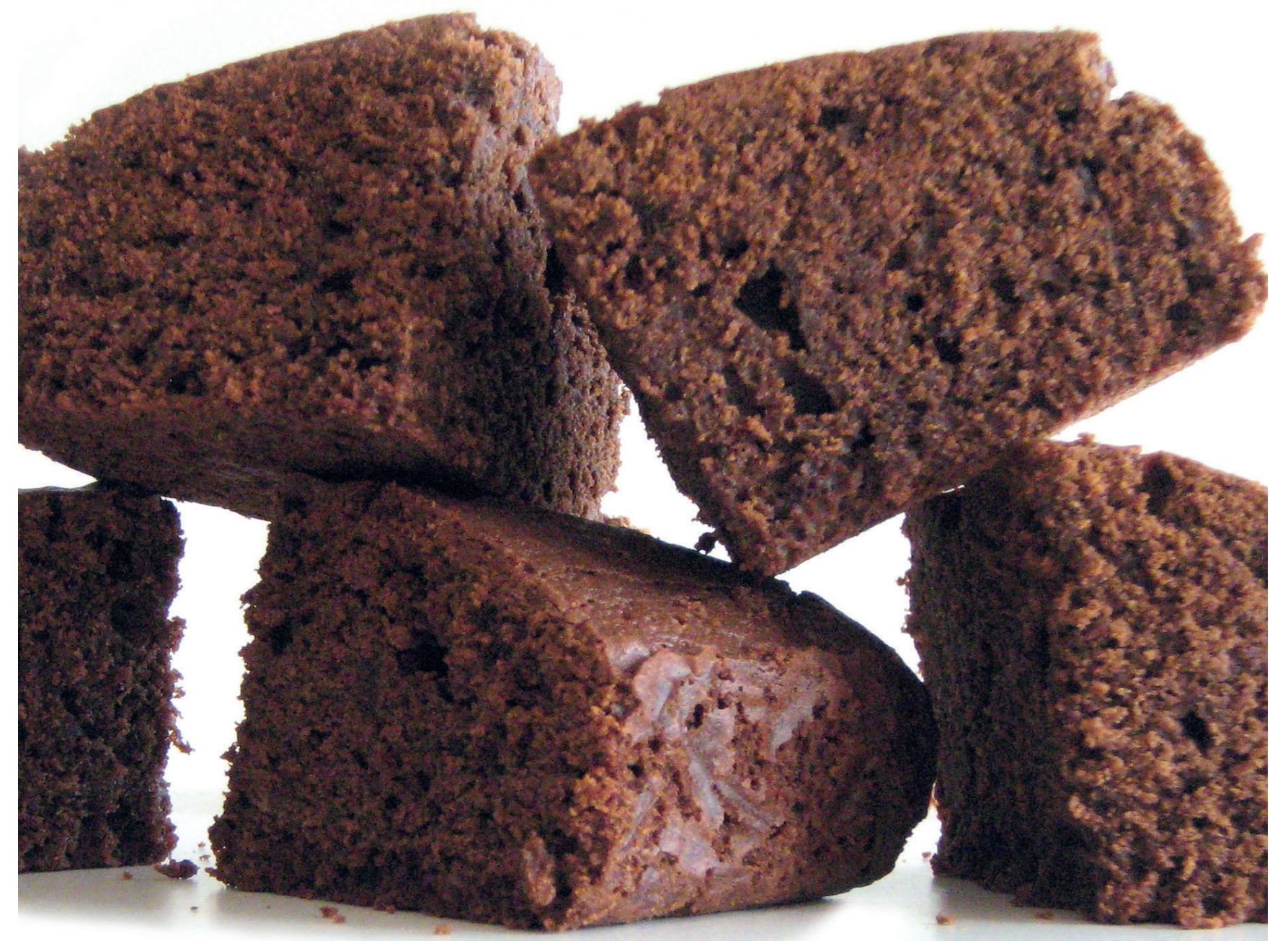




\section{CHAPTER 4}

NO DIFFERENCES IN SATIETY HORMONE AND ENDOCANNABINOID RESPONSE TO

PALATABLE VS NEUTRAL FOOD INTAKE

Suzanne E.M de Bruijn

Renger F. Witkamp

Cees de Graaf

Wilrike J. Pasman

Paul A.M. Smeets

Gerry Jager

Submitted in revised form. 


\begin{abstract}
BACKGROUND: Previous studies suggest that food palatability modulates endocannabinoid, ghrelin and pancreatic polypeptide (PP) responses across anticipatory, consummatory and postingestive phases. However, in some of these studies, participants knew in advance whether they were offered a palatable or a neutral food. Expected palatability, based on sensory cues such as sight and smell, likely affects anticipatory hormone responses and may also affect subsequent postingestive responses. In addition, post-ingestive endocannabinoid responses have only been measured at a limited number of time points and/or for a limited time period after tasting or ingesting a food, and insight into the full (pre-)prandial and post-prandial endocannabinoid response appears to be lacking.

AIM: To assess a complete profile of seven endocannabinoids and structural congeners, ghrelin and PP responses during anticipatory, consummatory and post-ingestive phases up to two hours after ingestion of a palatable versus a neutral food where palatability of the food was unknown until the first bite was taken.
\end{abstract}

MATERIALS AND METHODS: Twenty-five healthy males completed two test sessions that were two weeks apart during which they consumed either a palatable brownie or a neutral brownie in randomized order. The two brownies were selected based on pre-study individual liking scores. During the test sessions, participants did not know palatability of the brownie until their first bite. Before, during and up to two hours after brownie consumption, blood samples were drawn.

RESULTS: Endocannabinoid, ghrelin and PP profiles did not differ between the palatable and neutral brownie. Concentrations did not change during anticipation or consumption, but they did go down for most of the measured endocannabinoids and ghrelin after food intake. In addition, PP concentrations increased shortly after food intake.

CONCLUSIONS: These results suggest that actual palatability of a food does not affect endocannabinoid, ghrelin and PP responses to its intake. 


\section{Introduction}

The rewarding gustatory properties of a food can tempt us into eating even when we feel satiated. This so-called hedonic eating may contribute to overeating (Lowe and Butryn, 2007) and consequently to weight gain and obesity. Several studies point out a role for the endocannabinoid system (ECS) in the physiological mechanisms that underlie hedonic eating (for a review see Jager and Witkamp, 2014). The ECS comprises two cannabinoid receptors, $\mathrm{CB}_{1}$ and $\mathrm{CB}_{2}$, their endogenous ligands, so-called endocannabinoids of which anandamide (AEA) and 2-arachidonoylglycerol (2AG) are best known, and the enzymes responsible for synthesis and degradation of the endocannabinoids (Howlett, 2002).

Another important mediator of hedonic eating is ghrelin (Buss et al., 2014; Monteleone et al., 2013). Ghrelin is the only known orexigenic peptide, with plasma concentrations rising in anticipation of food intake and dropping after food intake (Cummings et al., 2001; Tschöp et al., 2001). Previous studies suggested that plasma concentrations of AEA follow a similar pattern (GattaCherifi et al., 2012). Similarly, plasma concentrations of the endocannabinoid congeners palmitoylethanolamide (PEA) and oleoylethanolamide (OEA) were shown to decrease after food intake (Monteleone et al., 2012; Rigamonti et al., 2015).

The rise and drop of ghrelin and endocannabinoid plasma concentrations seem to be modulated by both the expected and actual (experienced) palatability of a food. Anticipation of palatable food intake increased concentrations of ghrelin and the endocannabinoid 2-AG whereas anticipation of a neutral food did not (Monteleone et al., 2012; Rigamonti et al., 2015). Ghrelin and endocannabinoids also respond to tasting a food, so even before ingesting it. This has been studied in a modified sham-feeding experiment, in which food was tasted and chewed on, but was expectorated instead of swallowed. Ghrelin plasma concentrations were found to be higher after modified sham-feeding of a neutral pudding compared to a palatable, sweet tasting, and an aversive, bitter tasting, pudding (Mennella et al., 2015). In addition, 2-AG concentrations were higher in the palatable and neutral condition compared to the aversive condition (Mennella et al., 2015). In the same study, pancreatic polypeptide (PP) plasma concentrations were found to be increased after modified sham-feeding of the palatable pudding compared to the neutral and the aversive pudding. PP is released during mastication and after food ingestion, and has been suggested to decrease food intake (Khandekar et al., 2015).

Taken together, previous studies indicate that food palatability modulates endocannabinoid, ghrelin and PP plasma responses across anticipatory, ingestive and subsequent digestive and absorptive phases of food intake (see Figure 4.1 for a schematic overview). However, in previous studies, participants knew in advance whether they were offered a palatable or a neutral food. Expected palatability, based on sensory cues as sight and smell, likely affects the anticipatory preingestive response and may subsequently affect the post-ingestive response. In addition, postingestive endocannabinoid responses have only been measured at a limited number time points and/or for a limited time period after tasting or ingesting a food. Thus, we still lack a full endocannabinoid curve across food intake and post-ingestive phases (digestion and absorption). 


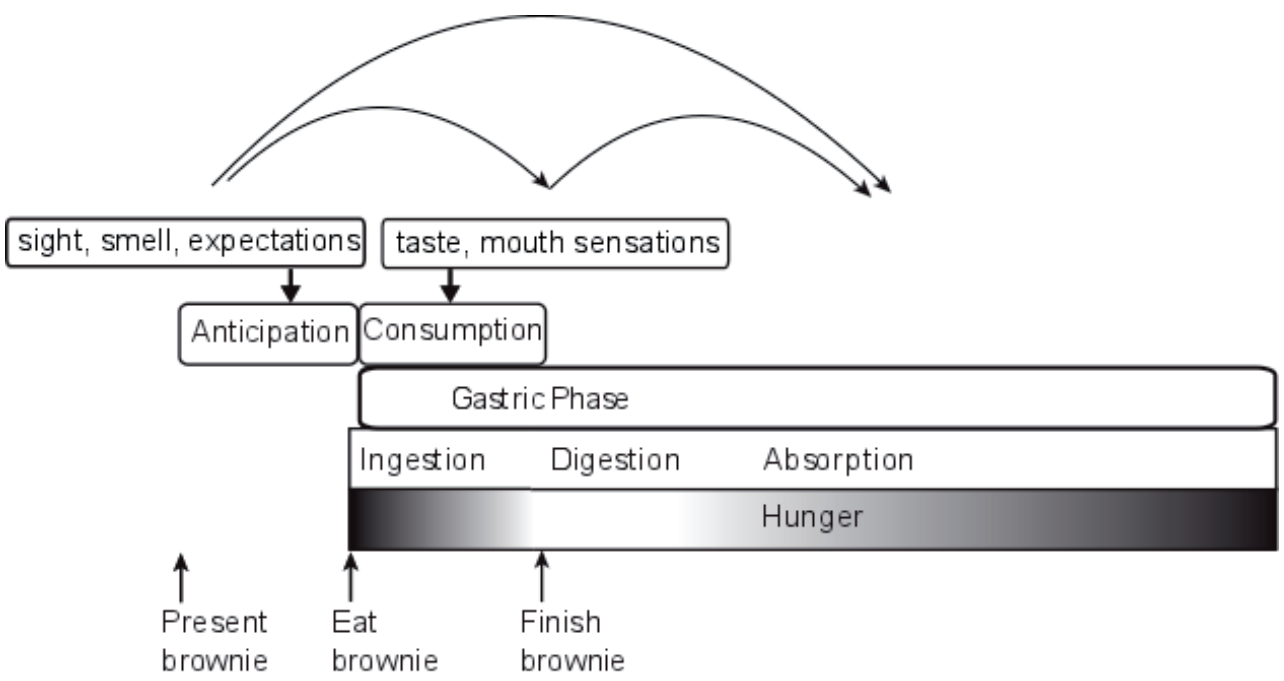

Figure 4.1. Schematic model of food intake behaviour as applicable in the current study. Before food consumption, the sight and smell of a food trigger anticipation, which is influenced by expectations. These expectations influence ingestive and post-ingestive responses. In addition, the taste of a food also influences post-ingestive responses. For hunger: the darker the colour, the higher feeling of hunger. Adapted with permission from Smeets et al., 2010.

Therefore, the aim of the current study was to assess a complete profile of seven endocannabinoids and related compounds, ghrelin and PP responses during anticipatory, consummatory and post-ingestive phases up to two hours after ingestion of a palatable versus a neutral food where palatability of the food was unknown until the first bite was taken.

To this end, we used chocolate brownies that only differed in salt content, and thus palatability, whereas they looked and smelled the same. Starting from the design by Monteleone and colleagues (2012), participants were first asked to look at and smell the brownie, before they could consume it. Participants were kept naïve about the study aim and did not know the palatability of the brownie until they took the first bite. In addition to endocannabinoids, ghrelin and PP, glucose concentrations and appetite ratings were assessed at different time points during the test session as control measures. These markers should respond to nutrient and caloric intake, but not to the palatability of the food. To further elucidate the post-ingestive endocannabinoid response, we not only measured AEA, 2-AG, OEA and PEA, but included three more endocannabinoid-related compounds that have been suggested to be relevant for food intake or induce cannabinomimetic behavioural effects, namely stearoylethanolamide (SEA; Terrazzino et al., 2004), dihomo- $\gamma$-linolenoylethanolamide (DLE; Barg et al., 1995), and docosahexaenoylethanolamide (DHEA; Barg et al., 1995; Friedman et al., 2016).

We hypothesised that exposure (viewing and smelling) to the palatable and the neutral brownie would not induce differences in any of the endocannabinoids, ghrelin or PP. After brownie intake, concentrations of 2-AG, ghrelin and PP were expected to be increased for the palatable compared to the neutral brownie. Brownie intake, independent of palatability, would lead to decreased concentrations of AEA, OEA and PEA after ingestion. 


\section{Methods}

\section{Participants}

Twenty-five males aged 21.8 ( $\mathrm{SD}=3.1$ ) years, with a mean BMI of $22.4(\mathrm{SD}=1.7) \mathrm{kg} / \mathrm{m}^{2}$ participated in the study. All participants were healthy (self-reported). To ensure participants were not anaemic and could safely undergo repeated blood sampling, their blood haemoglobin $(\mathrm{Hb})$ concentration was determined with a $\mathrm{Hb}$ analyser (HemoCue 201) at screening and had to be between 8.1 and $11.0 \mathrm{mmol} / \mathrm{L}$. As regular cannabis use influences the endocannabinoid system (SimSelley et al., 2006; Walter et al., 2013), participants were not allowed to use cannabis more than 12 times a year. Other exclusion criteria were restrained eating (Dutch Eating Behaviour Questionnaire

(DEBQ; Van Strien et al., 1986), score>2.90), lack of appetite, an energy restricted diet during the last two months, more than five kilograms body weight change during the last two months, smoking, drinking more than fourteen units of alcohol per week and not willing to consume or being allergic to products used in the study. Individuals who expressed interest in participating in the study where invited for an information and screening meeting at Wageningen University, during which they rated liking of six different brownies (see section 2.2) in random order on a 100point visual analogue scale (VAS). Only participants who rated one of the brownies as neutral, i.e., VAS-score between 40 and 60 , and another brownie as palatable, i.e., VAS-score $\geq 70$, were included in the study. Which two brownies were rated as neutral and palatable, and thus later presented in the test sessions could differ between participants. All participants provided written informed consent at the start of the information and screening meeting, and were debriefed at the end of the study. The study was approved by the Medical Ethical Committee of Wageningen University and was registered on clinicaltrials.gov as NCT03113474.

\section{Products}

Brownies were chosen as food product, as they are generally well liked. Palatability of the brownies was manipulated by adding 0-12.5 grams of salt to one kilogram of brownie dough, creating six different brownies that only differed in salt content. The salt affected palatability of the brownies, whereas the brownies looked and smelled the same. The brownies contained $62 \mathrm{~g}$ carbohydrates, $13 \mathrm{~g}$ fat, $5 \mathrm{~g}$ protein and $20 \mathrm{~g}$ water per $100 \mathrm{~g}$ and had an energy density of around $390 \mathrm{kcal} / 100 \mathrm{~g}$. A local bakery obtained brownie mix from Dawn Foods and prepared the brownies. After baking and cooling down, the brownies were stored in 100 gram serving portions in a freezer at $-18{ }^{\circ} \mathrm{C}$. On each study day, the needed brownies were defrosted at room temperature for at least two and a half hours, before being presented to the participants on paper plates.

\section{Procedures}

Before participants were included in the study, they attended an information and screening meeting. During this meeting, participants received a small piece ( $\sim 25 \mathrm{~g})$ of each brownie and tasted the different brownies in random order. Participants were instructed to taste one piece, to rate liking on a 100 point VAS and then rinse their mouth with water. There was a waiting time of at least one minute between tasting two pieces of brownies. To keep participants naïve to the real aim of the 
study, they were informed that they were participating in a study investigating the effects of eating on satiety hormones and that during the test sessions they would either receive two different brownies or the same brownie twice. Participants were also told they could receive either one of the six brownies, palatability was not mentioned in this explanation.

The study consisted of two test sessions that were two weeks apart. To limit the influence of recent food intake on outcome measures, participants were instructed to consume a standardized evening meal at home the evening before each test session and a standardized breakfast at the test location. The standardized evening meal was provided to the participants and consisted of a readymeal with chicken and soy sauce (2115 kJ / $495 \mathrm{kcal})$, a salad with eggs, potato and vegetables (931 $\mathrm{kJ} / 224 \mathrm{kcal})$ and a fruit salad $(598 \mathrm{~kJ} / 140 \mathrm{kcal})$. The total meal contained $859 \mathrm{kcal}$. Participants were instructed to consume the meal at their usual dinner time. They were instructed to eat the whole meal and to not consume any food or drinks afterwards, but to only drink water, tea or coffee. On the morning of each test session, participants came to the university where they received a standardized breakfast, consisting of two bread rolls (960 kJ / $225 \mathrm{kcal}$ ), butter (140 kJ / $34 \mathrm{kcal}$ ), one slice of ham (59 kJ / $14 \mathrm{kcal})$, jelly (166 kJ / $41 \mathrm{kcal})$ and a cup of tea. The total breakfast contained 314 kcal. Within 90 minutes after breakfast, an indwelling cannula was placed in the cephalic vein for blood sampling. Two hours after breakfast, a baseline blood sample was collected ( $\mathrm{T}=-5)$. Participants were then presented with $100 \mathrm{~g}$ of either the palatable or the neutral brownie in a counter-balanced order; 13 participants received the palatable brownie at the first test session and the neutral brownie at the second test session, whereas the other 12 participants received the brownies in the reversed order. Importantly, to minimize expectations, participants were told that they would receive one of the six brownies that they had tasted during the screening session and that they would receive either two different brownies or the same brownie twice in both test sessions. After brownie presentation, participants were instructed to look at and smell the brownie for five minutes, but to not eat it (anticipation phase). Three and five minutes later blood was collected $(\mathrm{T}=-2, \mathrm{~T}=0)$. Subsequently participants were instructed to eat the brownie within five minutes and additional blood samples were collected one, three and five minutes $(T=1, T=3, T=5)$ after participants started eating (consumption phase). Two participants did not consume the whole neutral brownie within five minutes, they ate 60 and 86 grams respectively. Data for both participants was included in analyses. Additional blood samples were collected at $\mathrm{T}=10,15,30,45$, 60, 90, and 120 minutes after participants started eating (post-ingestive phase). An appetite questionnaire was used to assess hunger, appetite for something sweet, and appetite for something savoury on a 100 point VAS. This questionnaire was completed at $\mathrm{T}=-5, \mathrm{~T}=0, \mathrm{~T}=5$ and $\mathrm{T}=7,10,15$, $30,45,60,90$, and 120 minutes after participants started eating. At $\mathrm{T}=5$, immediately after eating the brownie, participants also rated liking of the brownie. At time points where the appetite questionnaire and blood drawing coincided, the questionnaire was directly followed by the blood sampling. See Figure 4.2 for a schematic overview of the procedures starting at the first appetite questionnaire and blood sampling. Not all parameters were measured at all time points. Relevant time points were selected based on literature (Mennella et al., 2015; Monteleone et al., 2013; Witteman et al., 1994). See Table 4.1 for an overview. 
Table 4.1. Overview of parameters included in the different time points.

\begin{tabular}{llllllllllllll} 
Time: & -5 & -2 & 0 & 1 & 3 & 5 & 10 & 15 & 30 & 45 & 60 & 90 & 120 \\
\hline eCB & $\mathrm{X}$ & $\mathrm{X}$ & $\mathrm{X}$ & $\mathrm{X}$ & $\mathrm{X}$ & $\mathrm{X}$ & $\mathrm{X}$ & $\mathrm{X}$ & $\mathrm{X}$ & $\mathrm{X}$ & $\mathrm{X}$ & $\mathrm{X}$ & $\mathrm{X}$ \\
PP & $\mathrm{X}$ & & $\mathrm{X}$ & & & $\mathrm{X}$ & $\mathrm{X}$ & $\mathrm{X}$ & $\mathrm{X}$ & $\mathrm{X}$ & & & $\mathrm{X}$ \\
Ghrelin & $\mathrm{X}$ & $\mathrm{X}$ & $\mathrm{X}$ & $\mathrm{X}$ & & $\mathrm{X}$ & $\mathrm{X}$ & & $\mathrm{X}$ & $\mathrm{X}$ & $\mathrm{X}$ & $\mathrm{X}$ & $\mathrm{X}$ \\
Glucose & $\mathrm{X}$ & & $\mathrm{X}$ & $\mathrm{X}$ & $\mathrm{X}$ & $\mathrm{X}$ & $\mathrm{X}$ & & & $\mathrm{X}$ & & & $\mathrm{X}$
\end{tabular}

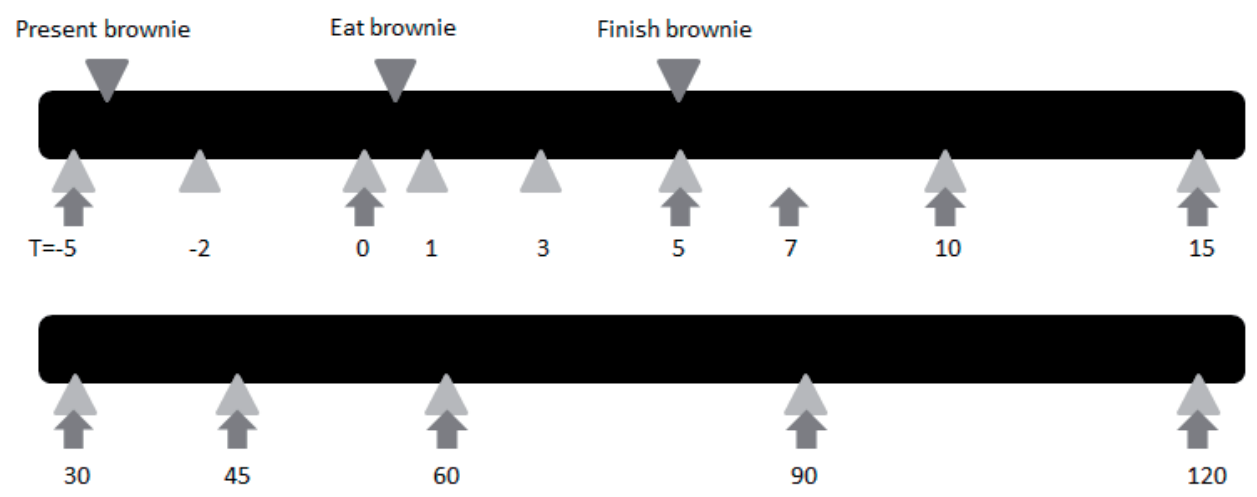

Figure 4.2. Schematic overview of a test session from the first blood sampling. Time (T) in minutes. The triangles indicate blood sampling, the arrows indicate appetite questionnaire. At time points the appetite questionnaire and blood drawing coincided, the questionnaire was directly followed by the blood sampling.

\section{Blood sampling and biochemical analyses}

For glucose measures, a syringe was used to collect a blood drop from the cannula. With the HemoCue 201 glucose concentrations in whole blood were determined. For ghrelin, endocannabinoids and PP measures, blood was collected in tubes with EDTA as anticoagulant. For ghrelin, at all times except $\mathrm{T}=3$ and $\mathrm{T}=15$, one tube with EDTA and added AEBSF (Millipore) was collected. After centrifuging, $\mathrm{HCl}$ was added to a final concentration of $0.05 \mathrm{~N}$. Immediately after collection, tubes were put on ice until being centrifuged at $1190 \mathrm{x}$ g for 10 minutes at $4{ }^{\circ} \mathrm{C}$. Plasma samples were portioned into aliquots and stored at $-80^{\circ} \mathrm{C}$. To one aliquot, phenylmethylsulfonyl fluoride (Sigma-Aldrich) and URB602 (Sigma-Aldrich) were added to a final concentration of 100 $\mu \mathrm{M}$ for both. This was done to inhibit breakdown of endocannabinoids.

Plasma concentrations of the endocannabinoids AEA and 2-AG, and the related $N$ acylethanolamines OEA, PEA, SEA, DHEA and DLE were measured using an LC-MS/MS technique as described elsewhere (Balvers et al., 2009). ELISAs were used to measure total ghrelin and PP concentrations (Millipore).

\section{Statistical analyses}

Results are expressed as means $\_$SD unless otherwise specified. Paired-samples T-tests were used to compare liking scores of the palatable brownie to the neutral brownie at screening and 
during the test sessions. Mixed model ANOVAs were used to analyse the appetite ratings and biochemical parameters. Condition, i.e., palatable versus neutral brownie, time and condition*time were included as fixed factors, and subject was included as a random factor. When relevant, posthoc analyses were performed using Bonferroni correction. Results were considered statistically different at $\mathrm{p}<0.05$. Statistical analyses were performed using SPSS version 22.0 (IBM Corporation, Armonk, New York, USA).

\section{Results}

\section{Liking of brownies}

The palatable brownies were liked more than the neutral brownies in the screening session $\mathrm{T}(1,24)=16.38, p<0.05$ (mean liking palatable brownie: $82 \pm 8$, neutral brownie: $51 \pm 5$ ). During the test sessions, liking of the palatable and neutral brownies was still significantly different, $T(1,24)=2.37$, $p<0.05$ (mean liking palatable brownie: $69 \pm 15$, neutral brownie: $60 \pm 22$ ), though the difference was smaller than expected based on the ratings during screening.

\section{Appetite ratings}

Hunger ratings did not differ between the two conditions, i.e., consuming a palatable or a neutral brownie, but did change over time (Figure 4.3). At $\mathrm{T}=-5$, mean hunger ratings were $54 \pm 16$ and $57 \pm 15$ for the palatable and the neutral condition, respectively. Reported hunger increased after exposure (viewing and smelling) to a brownie (mean hunger palatable brownie: $61 \pm 19$, neutral brownie: $66 \pm 15$ ), and decreased after consuming a brownie to a minimum at $\mathrm{T}=15$ (palatable brownie: $38 \pm 18$, neutral brownie: $45 \pm 22$ ), all $p<0.05$. At $\mathrm{T}=120$, reported hunger was comparable to before brownie exposure (palatable brownie: $59 \pm 22$, neutral brownie: $60 \pm 20$ ).

Similar to the reported hunger ratings, reported appetite for sweet increased after viewing and smelling a brownie (mean score at $\mathrm{T}=-5$ and $\mathrm{T}=0$, respectively palatable brownie: $54 \pm 20$ and $65 \pm 19$, neutral brownie: $53 \pm 19$ and $65 \pm 18$ ) and decreased after consuming a brownie, $p<0.05$ (mean score at $\mathrm{T}=5$ palatable brownie: $47 \pm 22$, neutral brownie: $45 \pm 25$ ), but appetite for sweet did not differ between conditions, $p>0.05$. Appetite for savoury did not increase after viewing the brownie, $p>0.05$ (mean score at $\mathrm{T}=-5$ and $\mathrm{T}=0$, respectively palatable brownie: $55 \pm 19$ and $53 \pm 18$, neutral brownie: $57 \pm 20$ and $59 \pm 22$ ), but it did decrease after brownie consumption, $p<0.05$ (mean score at $\mathrm{T}=5$ palatable brownie: $39 \pm 21$, neutral brownie: $51 \pm 25$.

\section{Glucose}

Before brownie consumption, which was two hours after breakfast, mean glucose concentrations were $4.3 \pm 0.7 \mathrm{mmol} / \mathrm{L}$ for the palatable brownie and $4.1 \pm 1.0 \mathrm{mmol} / \mathrm{L}$ for the neutral brownie. These values are comparable to the study by Monteleone, et al (2012). Glucose concentrations increased after consuming either brownie to a maximum at $\mathrm{T}=45, p<0.05$ (palatable brownie: $5.7 \pm 1.1 \mathrm{mmol} / \mathrm{L}$, neutral brownie: $5.6 \pm 0.8 \mathrm{mmol} / \mathrm{L}$ ) and did not differ between the palatable and the neutral brownie (Figure 4.3). 


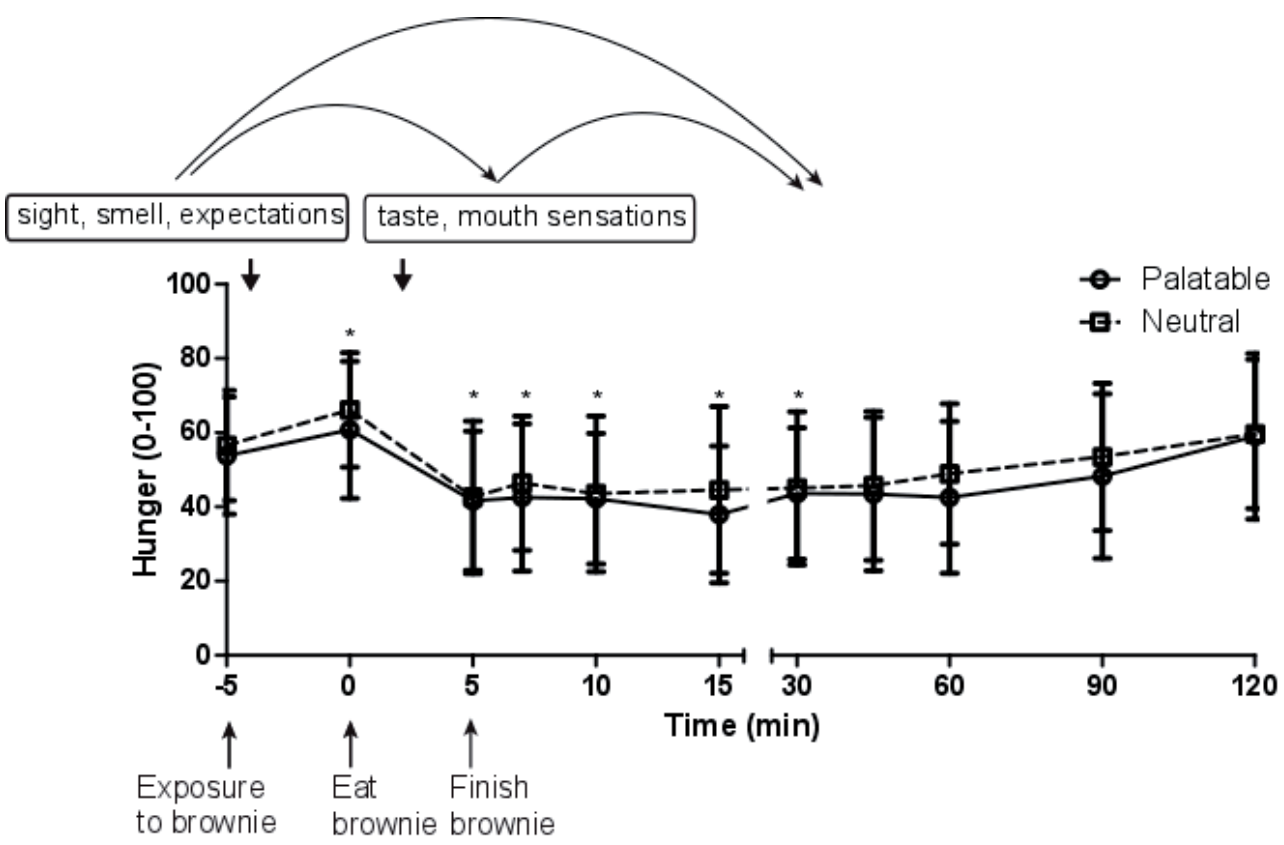

\begin{tabular}{|l} 
Anticipation Ingestion Digestion Absorption \\
Gastric Phase
\end{tabular}

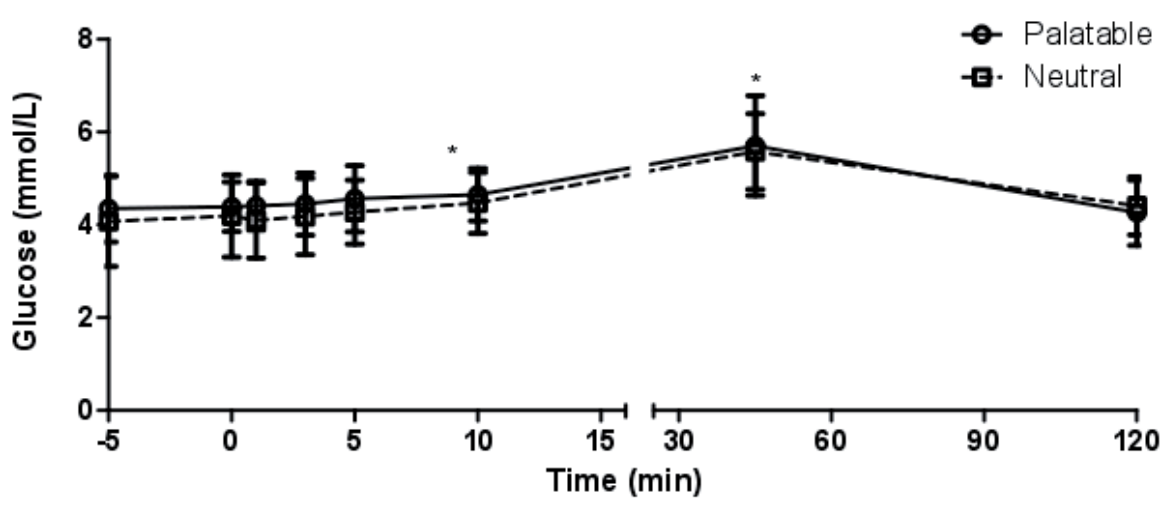

Figure 4.3. Hunger ratings (top panel) and glucose concentrations (bottom panel) during the test session (means $\pm \mathrm{SD}$ ). Anticipation increases feelings of hunger $(p<0.05)$, whereas food intake decreases feelings of hunger $(p<0.05) .{ }^{*}$ indicates significant difference from $\mathrm{T}=-5$.

\section{Endocannabinoids}

AEA concentrations were not modulated by palatability, $p>0.05$, and they did not change after exposure (viewing and smelling) to a brownie, $p>0.05$. However, they did decrease after intake of either brownie to a minimum at $\mathrm{T}=90, p<0.05$ (mean plasma concentrations at $\mathrm{T}=-5, \mathrm{~T}=0$ and 
$\mathrm{T}=90$ palatable brownie: $0.22 \pm 0.05,0.22 \pm 0.05 \mathrm{ng} / \mathrm{mL}$ and $0.13 \pm 0.05 \mathrm{ng} / \mathrm{mL}$; neutral brownie: $0.22 \pm 0.05,0.21 \pm 0.06 \mathrm{ng} / \mathrm{mL}$ and $0.13 \pm 0.05 \mathrm{ng} / \mathrm{mL}$ ) (Figure 4.4).

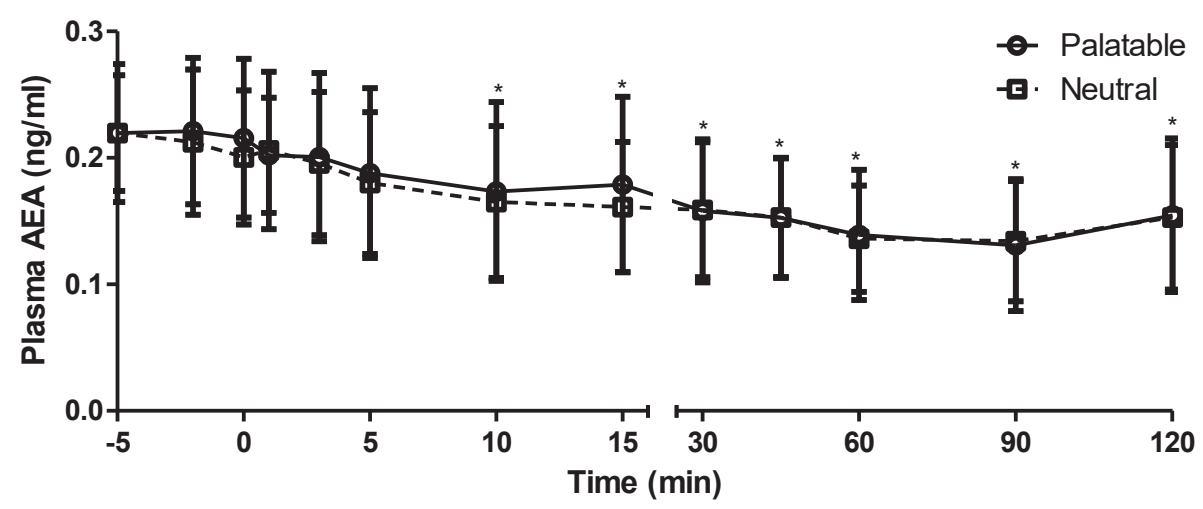

Figure 4.4. AEA plasma concentrations during the test session (means $\pm \mathrm{SD}$ ). ${ }^{*}$ indicates significant difference from $\mathrm{T}=-5$.

Similar results were obtained for DLE, OEA, PEA and SEA, i.e., no differences were found between the palatable and the neutral brownie, all $p>0.05$, and concentrations did not change during anticipation or consumption, all $p>0.05$. However, concentrations were decreased near the end of the test sessions, all $p<0.05$ (Supplementary Figure 4.1).

For DHEA and 2-AG, no differences were found between the palatable and the neutral brownie, both $p>0.05$, and contrary to the other endocannabinoids, concentrations did not change over time, both $p>0.05$ (Supplementary Figure 4.1).

\section{Ghrelin}

Ghrelin concentrations did not differ between conditions, $p>0.05$, but did decrease over time leading to significantly decreased concentrations towards the end of the test session, $p<0.05$ for $\mathrm{T}=60$ and $\mathrm{T}=90$ (mean palatable brownie: $577 \pm 265 \mathrm{pg} / \mathrm{ml}, 516 \pm 227 \mathrm{pg} / \mathrm{mL}$ and $524 \pm 251 \mathrm{pg} / \mathrm{mL}$, neutral brownie: $562 \pm 295 \mathrm{pg} / \mathrm{ml}, 515 \pm 277 \mathrm{pg} / \mathrm{mL}$ and $522 \pm 293 \mathrm{pg} / \mathrm{mL}$ for $\mathrm{T}=-5, \mathrm{~T}=60$ and $\mathrm{T}=90$, respectively) (Figure 4.5; see Supplementary Figure 4.2 for changes from baseline).

PP

Consuming a brownie lead to an immediate increase in PP concentrations $p<0.05$ (mean concentrations palatable brownie: $248 \pm 180 \mathrm{pg} / \mathrm{mL}$ and $376 \pm 218 \mathrm{pg} / \mathrm{mL}$, neutral brownie: $200 \pm 203$ $\mathrm{pg} / \mathrm{mL}$ and $352 \pm 237 \mathrm{pg} / \mathrm{mL}$ for $\mathrm{T}=0$ and $\mathrm{T}=5$ respectively), but this did not differ between the palatable and the neutral brownie, $p>0.05$ (Figure 4.5; see Supplementary Figure 4.2 for changes from baseline). 

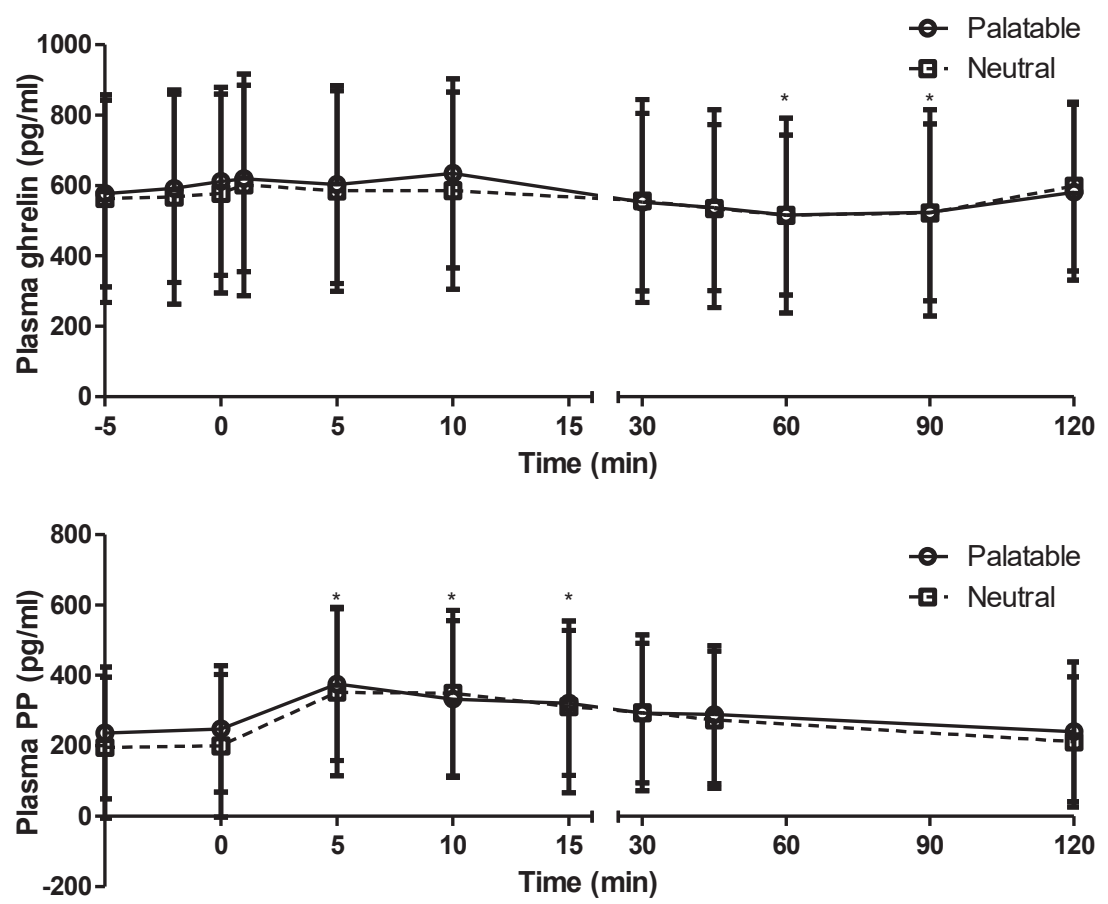

Figure 4.5. Plasma ghrelin (top panel) and PP (bottom panel) concentrations during the test sessions (means $\pm \mathrm{SD}) .{ }^{*}$ indicates significant difference from $\mathrm{T}=-5$.

\section{Discussion}

To the best of our knowledge, the current study is the first to cover an elaborate profile of endocannabinoids, ghrelin and PP responses during anticipatory, consummatory and post-ingestive phases up to two hours after ingestion for a palatable versus a neutral food where palatability of the food was unknown until the first bite was taken. As expected, anticipation (viewing and smelling) of either brownie did not induce differences in endocannabinoid, ghrelin or PP plasma concentrations. Contrary to our hypotheses, results indicate no differences in endocannabinoid, ghrelin or PP responses between the palatable and the neutral brownie after brownie intake. We did find a decrease in plasma concentrations of most $N$-acylethanolamides after food intake, independent of palatability.

In the current study, anticipation (viewing and smelling) did not affect endocannabinoid, ghrelin and PP concentrations. Anticipation of the palatable and the neutral brownie significantly increased feelings of hunger and appetite for sweet. Appetite for savoury did not change during anticipation. This is line with previous studies in which participants reported increased appetite for the food they were exposed to (see e.g. Fedoroff et al., 2003; Ramaekers et al., 2016). After brownie consumption, hunger concentrations, appetite for sweet and appetite for savoury decreased. Toward 
the end of the test session, which was close to normal lunch time in The Netherlands, participants started to feel more hungry again.

Importantly, participants were told that they would receive either one of the six brownies they tasted during the screening session and that they would receive either two different brownies or the same brownie twice in both test sessions. This led to insecurity about brownie palatability until taking the first bite. As we did not find differences between the palatable and the neutral condition for any of the plasma parameters and appetite-related questions during anticipation, we conclude that we were successful in keeping participants insecure about brownie palatability.

Ghrelin and 2-AG did not differentially respond to consumption of the palatable versus the neutral brownie. This finding is contrary to previous studies which suggested that anticipation of and consuming a palatable versus a neutral food increases 2-AG and ghrelin plasma concentrations (Monteleone et al., 2016; Monteleone et al., 2012; Rigamonti et al., 2015). These discrepancies might be caused by differences in expected palatability. In the current study, participants did not know the palatability of the brownie that they were about to consume until they took the first bite, whereas in previous studies palatability was known beforehand. Indeed, anticipation of palatable food has previously been shown to affect 2-AG and ghrelin concentrations (Monteleone et al., 2012; Rigamonti et al., 2015), and may have affected post-ingestive concentrations as well. Together, these findings suggest that expected, but not actual palatability affects 2-AG and ghrelin concentrations. This points to a role for 2-AG and ghrelin in motivation to eat and not in liking of a food during consumption. Indeed, ghrelin concentrations rise before meal initiation and correlate with selfreported feelings of hunger (Cummings et al., 2004; Cummings et al., 2001). Exposure to palatable foods triggers a rise in ghrelin concentrations (Rigamonti et al., 2015; Schussler et al., 2012) and in this way may stimulate food intake.

The role for ghrelin and the ECS in anticipation of food intake is substantiated by results from brain imaging studies. Ghrelin concentrations are related with brain activation in rewardrelated areas in response to pictures of food, which cue food anticipation (Kroemer et al., 2013). Modulating ECS activity alters reward-related activation in response to the sight of chocolate (Horder et al., 2010; Tudge et al., 2015). Moreover, in animals it was found that ECS activation leads to increased motivation to obtain a palatable sucrose solution (Higgs et al., 2003), whereas ECS inhibition specifically decreases reward value of palatable foods (Higgs et al., 2003; Rasmussen et al., 2012). Future studies could investigate whether the ECS also modulates motivation to obtain food in humans.

Ghrelin and the endocannabinoids AEA, OEA, PEA, SEA and DLE were decreased after food intake, whereas DHEA and 2-AG did not respond to food intake. For ghrelin, AEA, OEA and PEA, this is in line with previous studies (Cummings et al., 2001; Gatta-Cherifi et al., 2012; Monteleone et al., 2012; Rigamonti et al., 2015; Tschöp et al., 2001). Our finding that 2-AG is not affected by food intake is in line with a previous study (Gatta-Cherifi et al., 2012). For SEA, DLE and DHEA, the current study is the first to report pre- and post-ingestive profiles. SEA and DLE followed similar patterns as AEA, OEA and PEA, and might therefore play a role in food consumption in humans. More studies that measure these $N$-acylethanolamides after food intake are necessary to confirm the current results. 
As expected, PP concentrations rose in response to food intake. However, there were no differences between palatable and neutral food intake. A previous study showed that PP concentrations were higher after tasting, but not swallowing, a palatable versus a neutral food (Mennella, et al., 2015). In the current study, participants swallowed the food, which caused a postingestive response that may have overruled the effects of taste. PP does not play a role in anticipation of food intake, but the role of PP is more related to tasting a food and food ingestion. Previous studies showed that PP differentially responds to macronutrient content of foods (Crystal and Teff, 2006; Witteman et al., 1994), which might be more important than palatability.

In the current study, we measured plasma concentrations of endocannabinoids. The exact function of plasma endocannabinoids remains to be elucidated. It has been suggested that plasma endocannabinoids are a result of spill-over from tissues like the brain and gut (Hill et al., 2008; Matias et al., 2012), however, it is unknown to what extent plasma concentrations reflect endocannabinoid concentrations in these other tissues. A previous study suggested a link between plasma endocannabinoid concentration and feelings of appetite (Friedman et al., 2016).

An important strength of the current study is that we used one food product, i.e., brownie, which we modulated to only change liking (taste). Because we used one food product, macronutrient content and energy density were similar for both the palatable and the neutral food. Participants tasted and rated liking for six different brownies and for each participant the palatable and neutral brownie were selected based on individual liking. Even though brownies were selected to be experienced as palatable and neutral, the difference in liking ratings between the palatable and the neutral brownie during the test sessions was smaller, though still significant, than in the screening session. A possible explanation for this is that participants rated liking of one bite during the screening session and of 100 grams during the test session. In future studies it is recommended to also ask liking after one bite during the test session.

In addition, to gain more insight into the respective roles of expected and actual palatability on the endocannabinoid, ghrelin and PP response to food intake, a future study could match and mismatch expected and actual palatability. Expected palatability has previously been shown to induce differences in 2-AG and ghrelin (Monteleone et al., 2012). If actual palatability does not affect these responses, then matched or mismatched foods should not induce different responses. Future studies that assess post-ingestive endocannabinoid concentrations should adhere to a similar sampling schedule to confirm the current results. In addition, it could be of interest to assess different types of foods, i.e. more snack-like versus complete meals, to investigate whether there are differences in endocannabinoid responses to different food types.

In conclusion, food intake decreases concentrations of ghrelin, AEA, OEA, PEA, SEA, and DLE, but does not affect 2-AG or DHEA. In addition, it increases concentrations of PP. However, creating insecurity about the palatability of a food before the first bite is taken, blocks differences in endocannabinoid, ghrelin and PP responses during anticipatory, consummatory and postingestive phases of consuming a palatable versus a neutral brownie. 


\section{Acknowledgements}

We would like to thank Anouk Andeweg, Henriëtte Fick-Brinkhof, Diana Emmen-Benink, Els Siebelink and Jantien Takens for their help in carrying out the study, and Richard Bas, Maarten Hekman, Frederique van Holthoon and Nhien Ly for their assistance with plasma analyses.

Research presented in this publication was financially supported by the Graduate School VLAG. 
Supplementary Figure 4.1: Plasma concentrations of the endocannabinoids 2-AG (top left panel), DHEA (top right panel), DLE (middle left panel), OEA (middle right panel), PEA (bottom left panel) and SEA (bottom right panel) during the test sessions (means $\pm \mathrm{SD}$ ). ${ }^{*}$ indicates significant difference from $\mathrm{T}=-5$.
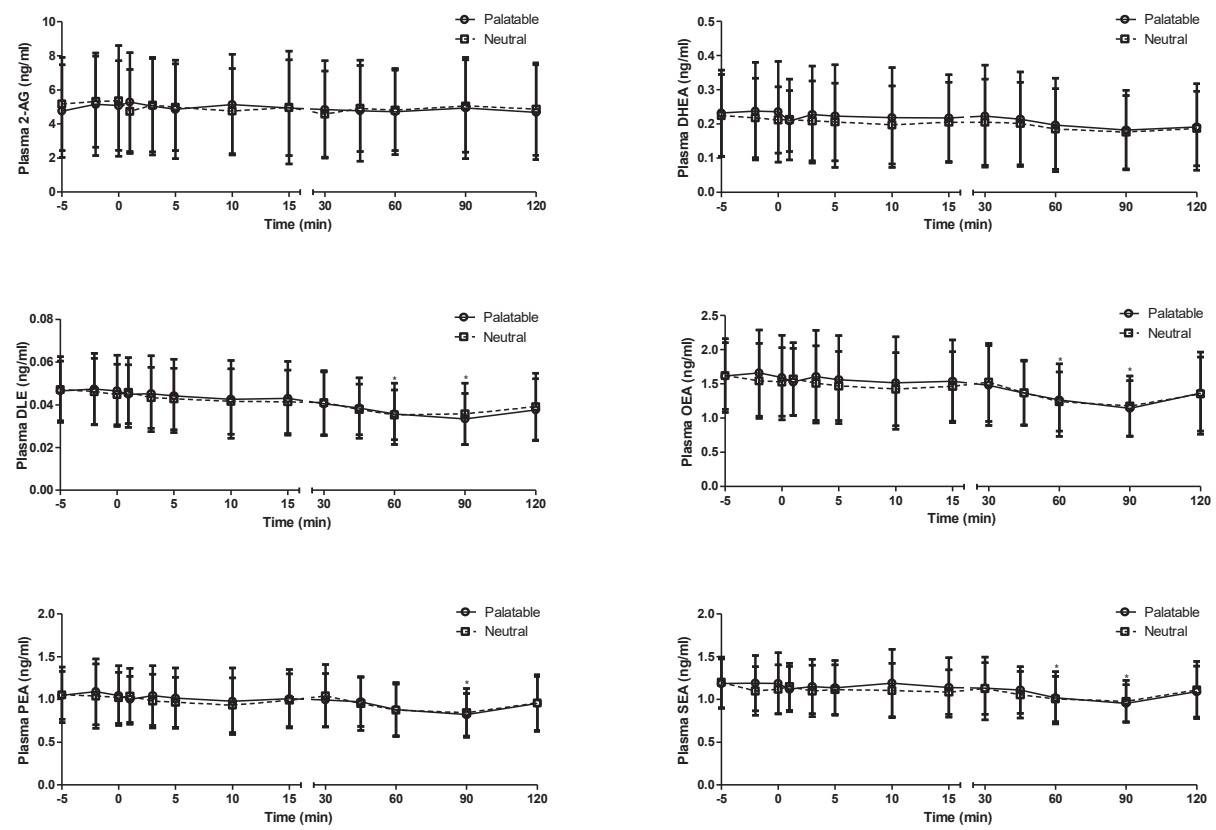
Supplementary Figure 4.2. Differences from baseline for plasma ghrelin (top panel) and PP (bottom panel) during the test sessions (means $\pm \mathrm{SD}$ ). ${ }^{*}$ indicates significant difference from $\mathrm{T}=-5$.
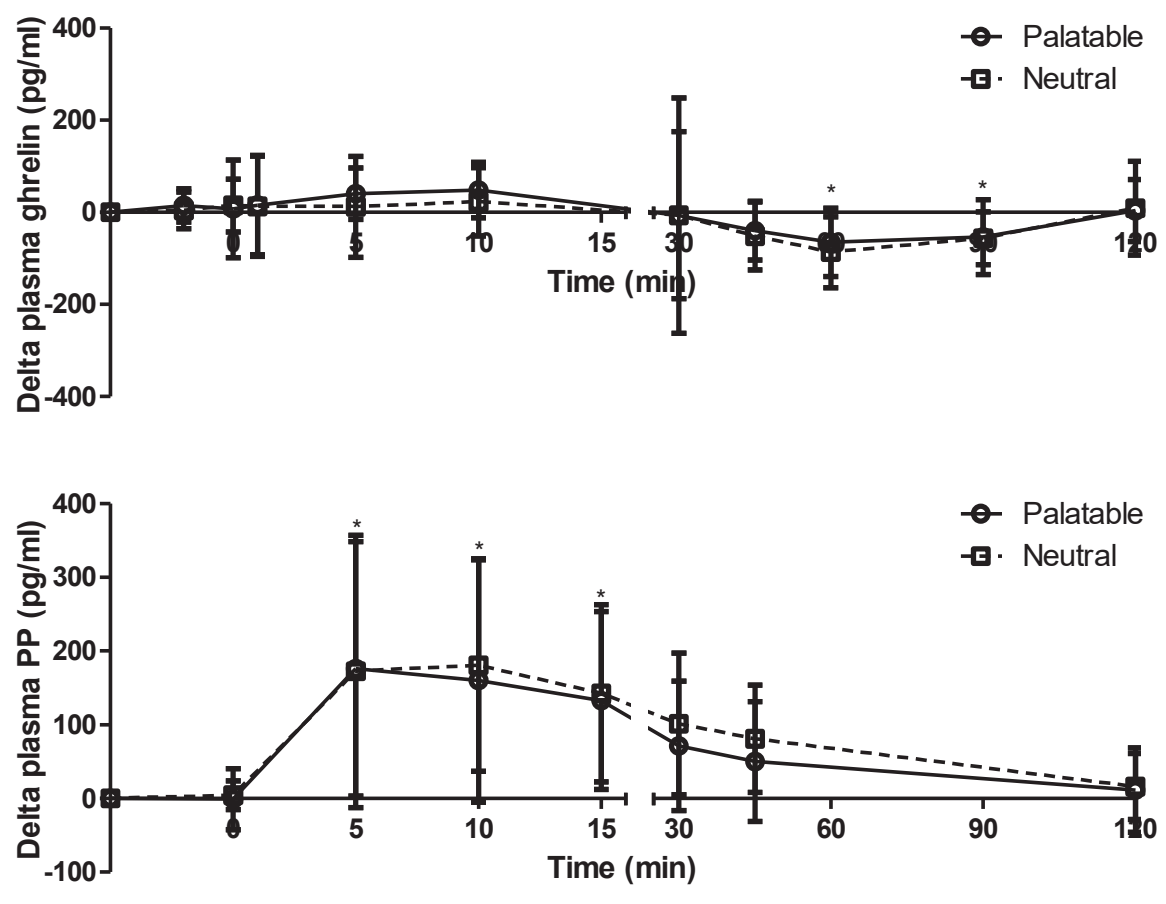


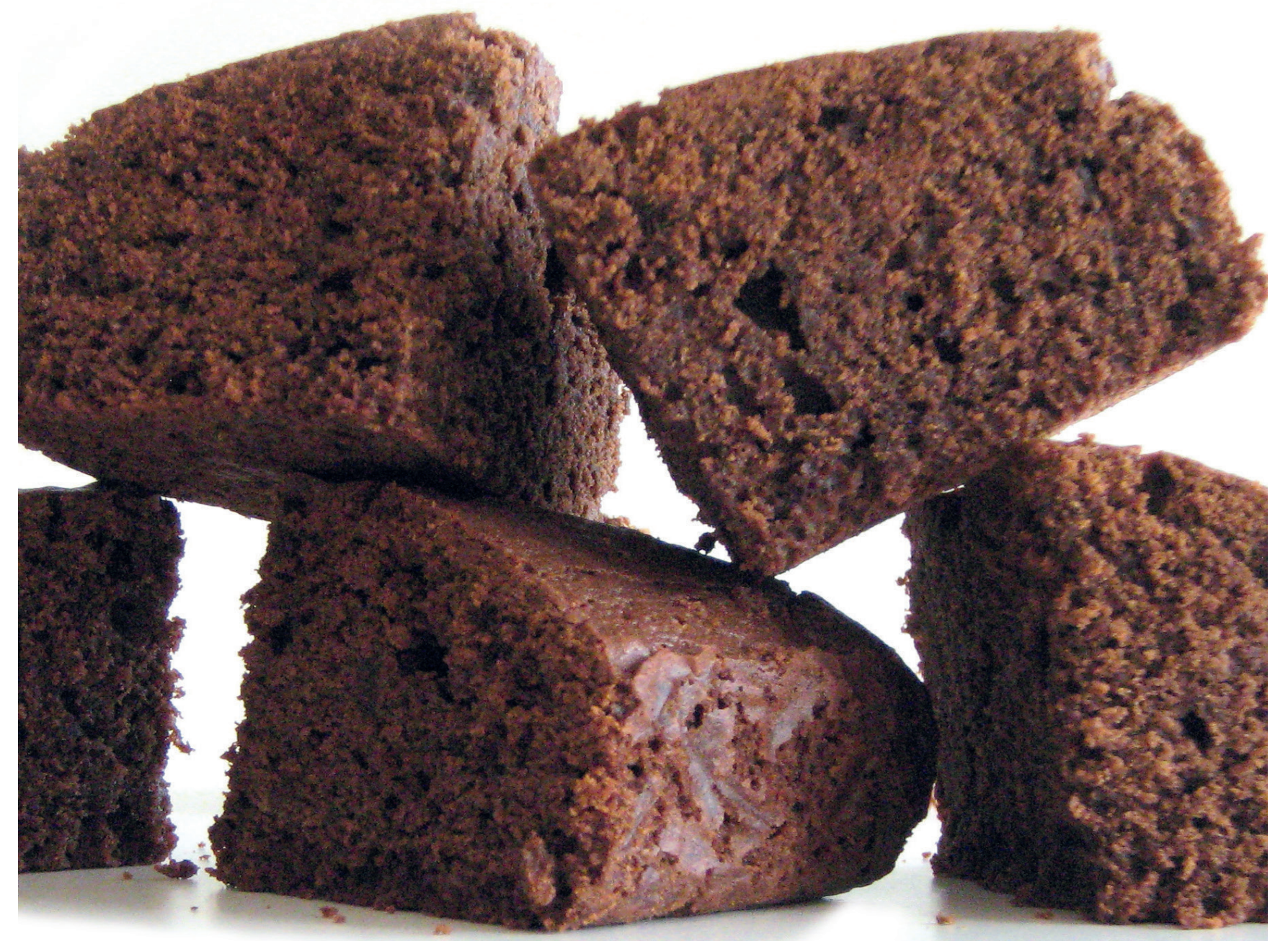




\section{CHAPTER 5}

DECREASED PREFERENCE FOR HIGH-CARBOHYDRATE AND HIGH-FAT FOODS AFTER ROUX-EN-Y GASTRIC BYPASS SURGERY

Suzanne E.M de Bruijn

Harriët F.A. Zoon

Cees de Graaf

Sanne Boesveldt

Gerry Jager

In preparation. 


\begin{abstract}
BACKGROUND: After Roux-en-Y gastric bypass (RYGB) surgery, patients report a shift in food intake from high-energy to low-energy foods, which corresponds with reported decreased desire to eat high-energy foods. However, follow-up over time has been limited and food preferences have not been measured in a forced-choice paradigm. An important advantage of forced-choice paradigms over liking questionnaires is that products from different food categories are directly compared, and this provides more insight into food choices.

AIM: To assess the effect of RYGB on food preferences for four macronutrient and two taste categories from pre-surgery to two months and one year after surgery.

METHODS: Seventy-nine RYGB patients completed an online food preference task, the Macronutrient and Taste Preference Ranking Task, two weeks before surgery, and two months and one year after surgery.

RESULTS: Preference for high-carbohydrate and high-fat foods decreased, whereas preference for low-energy foods increased after surgery. Preference for sweet was decreased at two months, but not at one year after surgery. Compared with pre-surgery, liking of the high-carbohydrate and highfat foods decreased at two months and one year after surgery, whereas liking of low-energy foods was increased at one year after surgery only. Loss in BMI at one year after RYGB surgery was positively correlated with changes in preference for high-carbohydrate foods at one year after RYGB surgery.

CONCLUSION: RYGB induces decreased preference for high-carbohydrate and high-fat foods, and increased preference for low-energy foods at two months and one year after surgery. These changes seem to be related to changes in liking of high-carbohydrate and high-fat foods. The change in preference for high-carbohydrate foods is significantly correlated with loss of BMI.
\end{abstract}




\section{Introduction}

Currently, the most effective long-term treatment for obesity is bariatric surgery, with Roux-en-Y gastric bypass (RYGB) surgery regarded as the golden standard (Buchwald et al., 2004; Gloy et al., 2013). In RYGB, a small stomach pouch is created, and then attached to the jejunum. After surgery, food bypasses the distal stomach, duodenum and proximal part of the jejunum. Importantly, other surgical techniques, like vertical-banded gastroplasty and sleeve gastrectomy, only create a smaller stomach, but do not bypass the upper part of the intestine (Hng and Ang, 2012).

After RYGB, patients not only lose weight, but they also change their dietary patterns. Patients have proportionally lower intake of fatty foods (Mathes and Spector, 2012; Meillon et al., 2013) and some studies suggest increased consumption of fruit and vegetables (Laurenius et al., 2013; Ullrich et al., 2013). These changes in food choice and dietary patterns correspond with changes in measures of food reward (le Roux et al., 2011). Desire to eat high-energy foods was found to be decreased one month after surgery, whereas desire to eat low-energy foods did not change (Ochner et al., 2012b). In a progressive-ratio paradigm, patients were less willing to work for a chocolate reward eight weeks after compared to two weeks before RYGB. Effort for a vegetable reward did not change after surgery (Miras et al., 2012). After a longer period of time, median eight months after surgery, RYGB patients rated high-calorie foods as less appealing compared to BMI-matched participants (Scholtz et al., 2014). More than one year after surgery, RYGB patients reported a decreased urge for obtaining palatable foods (Schultes et al., 2010; Ullrich et al., 2013). Importantly, not all patients report decreased enjoyment of highly palatable foods and similarly, only part of the patients reports increased enjoyment of healthy foods. This higher enjoyment of healthy dietary behaviour is associated with successful weight loss after two years (Vuorinen et al., 2016).

Thus far, most studies assessed food preferences at a single time-point and only a few studies have performed follow-up of changes in food reward or food intake over time. These studies suggest changes immediately after RYGB that last for twelve to eighteen months, but after two years, eating habits start to return to pre-surgery habits (Cushing et al., 2013; Laurenius et al., 2013). This return to pre-surgery habits might be related to weight regain that starts to occur after approximately two years in a subset of patients (Courcoulas et al., 2013; Magro et al., 2008).

Although several studies assessed the changes in food reward after RYGB (Cushing et al., 2013; le Roux et al., 2011; Ochner et al., 2012b), food preferences have not been measured in a forced-choice or ranking paradigm. An important advantage of forced-choice paradigms over liking questionnaires is that products from different food categories are directly compared. Two foods might be liked equally, but in a forced-choice procedure, preference for one food over the other may become apparent. The relative preference for one food over another can provide insight into motivation for the chosen food over the non-chosen food (Finlayson et al., 2008).

Therefore, the aim of current study is to assess the effect of RYGB on relative food preferences for four macronutrient and two taste categories from pre-surgery to two months and one year after surgery. To reach this aim, food preferences were measured using the Macronutrient and Taste Preference Ranking Task (MTPRT; de Bruijn et al., 2017). In this task, participants are asked to rank four food products from different macronutrient and taste categories on how much they desire to eat those products at that moment. We hypothesised that at both time points after 
RYGB, preference for foods high in carbohydrate and fat would be decreased, and preference for low-energy foods would be increased.

\section{Methods}

\section{Participants}

Seventy-nine participants (12 males) participated in the current study. Mean age at surgery was 42.3 years $(\mathrm{SD}=8.9)$ and mean $\mathrm{BMI}$ before surgery was $43.6 \mathrm{~kg} / \mathrm{m}^{2}(\mathrm{SD}=5.2)$. All participants were enlisted to undergo RYGB surgery at Rijnstate hospital, Arnhem, the Netherlands. Requirements for the surgery were a Body Mass Index (BMI) of $>40 \mathrm{~kg} / \mathrm{m}^{2}$ or $>35 \mathrm{~kg} / \mathrm{m}^{2}$ with comorbidity that was expected to improve after surgically-induced weight loss, long-lasting obesity ( $>5$ years), proven failed attempts to lose weight in a conventional way, and intention to adhere to a postoperative follow-up program. Inclusion and screening of participants took place at Rijnstate hospital, on the same day as the standard intake for the surgery. Patients were individually informed about the study and asked to participate. When patients expressed interest to participate, they signed informed consent and completed a short questionnaire to check inclusion and exclusion criteria. Participants had to be between 18 and 65 years of age, and were excluded when they were allergic to products used in the MTPRT, reported a lack of appetite, or reported difficulties swallowing or eating. Participants received a gift card for their contribution. The protocol was approved by the Medical Ethical Committee of Wageningen University and was registered on clinicaltrials.gov as NCT02068001.

\section{Macronutrient and Taste Preference Task}

The MTPRT (de Bruijn et al., 2017) is a computerized task to assess liking of and food preferences for four macronutrient categories, i.e., high-carbohydrate, high-fat, high-protein and low-energy, and two taste categories, i.e., sweet and savoury. The MTPRT includes pictures of 32 food products, equally divided over the macronutrient categories. Four out of the eight products per macronutrient category are sweet and the other four products are savoury, with the exception of the high-protein category that consists of eight savoury products. Participants first rate liking of all 32 food products on a 100 point visual analogue scale (VAS) and are then presented with 28 arrays of four products. They are asked to rank the four products according to how much they desire to eat those products at that moment. The first sixteen rankings are focused on macronutrients and the four pictures represent products from all four macronutrient categories. The last twelve rankings are focused on taste and include two sweet and two savoury pictures, which come from two macronutrient categories. High-protein products are not used in this part of the task. Based on the rankings, relative preference scores for products from different macronutrient and taste categories, i.e. high-carbohydrate, high-fat, high-protein and low-energy, and sweet and savoury are calculated as described elsewhere (de Bruijn et al., 2017). Preference scores for the macronutrient categories can range from 1 to 4 , with a higher score indicating a higher preference for a category. A score of 1 indicates that all products from that category were ranked last; a score of 4 indicates all products from that category were ranked first. As each ranking in the taste section comprises two sweet and two savoury products, preference scores for sweet and savoury can range from 1.5 to 3.5. For both 
macronutrient and taste categories, a score of 2.5 indicates no relative preference. Because preference scores for sweet and savoury are each other's opposite, we only report preference scores for sweet in this article (de Bruijn et al., 2017).

\section{Procedure}

The online version of the MTPRT was presented in EyeQuestion software (Logic8 BV). Participants received an e-mail with a link to the MTPRT two weeks before, two months after and one year after surgery. They were asked to complete the task in one go, which took about fifteen minutes. In addition, participants were weighed before, and at four weeks and one year after surgery as part of standard care.

Of the 79 participants who completed the online MTPRT before surgery, 66 participants completed the online MTPRT two months after surgery and 46 participants completed the online MTPRT one year after surgery.

Of the 79 participants who completed the online MTPRT before surgery, weight was available of 78 participants before surgery, 74 participants at four weeks after surgery and of 43 participants at one year after surgery.

\section{Data analysis}

Data are presented as means \pm standard deviation (SD) unless otherwise specified. The rankings were used to calculate relative preference scores for the different macronutrient and taste categories as explained elsewhere (de Bruijn et al., 2017). Liking scores per category were calculated by taking the average liking of all products within a specific category. For example, liking ratings of all high-carbohydrate products were added up and divided by eight to calculate average liking of high-carbohydrate.

Data was analysed using IBM SPSS 22 (IBM Corporation, Armonk, New York, USA). Results were considered statistically significant at $p<0.05$.

Mixed models analysis was used to assess weight loss and differences in preference scores between time points. Time was added as fixed effect. Compound symmetry was chosen as covariance structure. When a statistically significant main effect was found, post-hoc analyses were performed using Bonferroni correction.

To assess specific preferences within time points, Friedman ANOVA was used to compare preference scores for the macronutrients. When significant effects were found, Wilcoxon signed rank tests were used as post-hoc with Bonferroni correction. A Wilcoxon signed rank test was also used to assess relative preference for sweet or savoury taste.

To gain more insight into the meaning of the relative preference scores, liking scores were compared using mixed models analyses with time as fixed effect and participant ID as random factor. When a statistically significant main effect was found, post-hoc analyses were performed using Bonferroni correction. To assess differences in liking within time points, one-way ANOVA was used to compare liking of the macronutrients. When significant effects were found, post-hoc analyses were performed using Bonferroni correction. A paired-samples T-test was used to asses differences in liking for sweet or savoury taste within time points. 
To assess correlations between BMI loss and changes in food preferences, Spearman's rank correlation coefficient was used. For this, change in BMI and change in preference scores from before surgery to two months and to one year after RYGB surgery were used.

\section{Results}

\section{Weight loss}

Before surgery, participants had a mean weight of $126 \pm 18 \mathrm{~kg}$ and a mean BMI of $43.6 \pm 5.2$ $\mathrm{kg} / \mathrm{m}^{2}$. After surgery, participants lost weight to a mean weight of $116 \pm 18 \mathrm{~kg}$ at four weeks and $82 \pm 15 \mathrm{~kg}$ at one year after RYGB surgery. BMI decreased to $40.1 \pm 5.4 \mathrm{~kg} / \mathrm{m}^{2}$ at four weeks and $28.7 \pm 4.8 \mathrm{~kg} / \mathrm{m}^{2}$ at one year after RYGB surgery, $p<0.05$.

\section{Ranking}

Food preference scores for the four macronutrient categories are shown in Figure 5.1. Preference for high-carbohydrate and high-fat foods were decreased two months and one year after surgery compared to before, all $p<0.05$. Preference for low-energy foods was increased two months and one year after surgery, $p<0.05$. Preference for high-protein foods did not change over time, $p>0.05$.

A. Carbohydrate

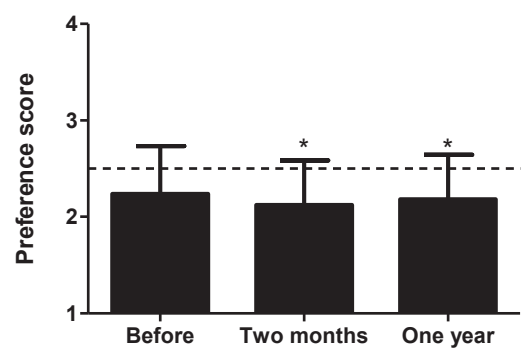

C. Protein

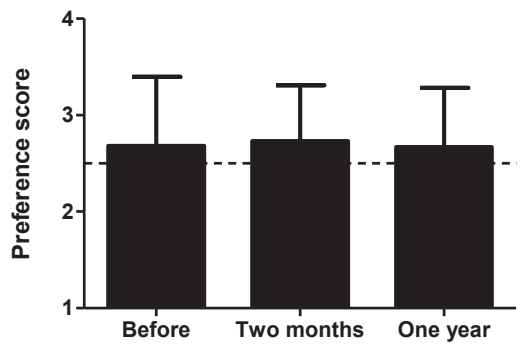

B. Fat

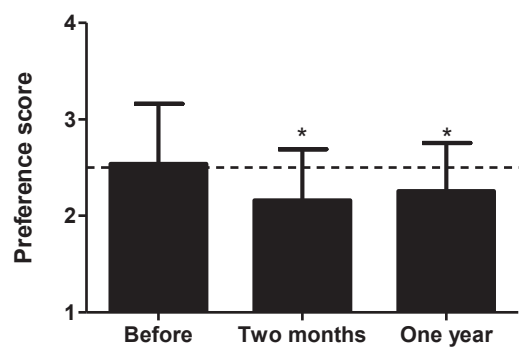

D. Low-energy

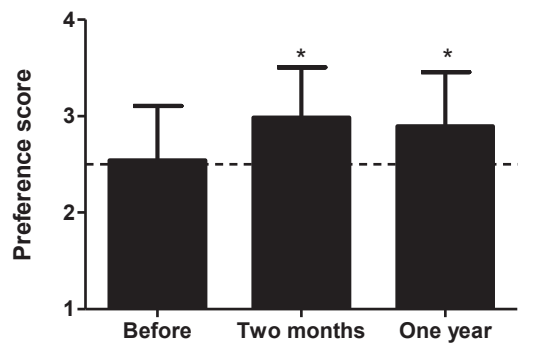

Figure 5.1. Preference scores for high-carbohydrate (A), high-fat (B), high-protein (C) and lowenergy (D) before, two months after and one year after RYGB surgery. Means \pm SD. ${ }^{*}$ indicates significant change compared to before surgery at $\mathrm{p}<0.05$ level. The dotted line represents chance level. 


\section{Preference for sweet}

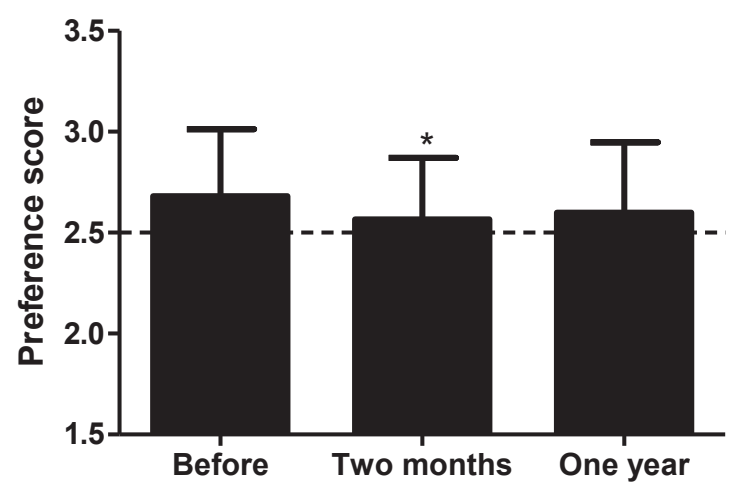

Figure 5.2. Preference for sweet before, two months after and one year after RYGB surgery. Means \pm SD. ${ }^{*}$ indicates significant change compared to before surgery at $\mathrm{p}<0.05$ level. Dotted line represents chance level.

Before surgery, participants had a lower preference for high-carbohydrate foods compared to the other macronutrients, all $p<0.05$. Two months after surgery, participants had a preference for low-energy foods over the other macronutrient categories, all $p<0.05$. In addition, preference for high-protein foods was higher than preference for high-carbohydrate and high-fat foods, both $p<0.05$. Preference for high-carbohydrate and high-fat foods did not differ, $p>0.05$. At one year after surgery, participants showed a preference for low-energy and high-protein foods over highcarbohydrate and high-fat foods, all $p<0.05$.

Preference for sweet is shown in Figure 5.2. Preference for sweet foods changed over time, $p<0.05$. Post-hoc analyses showed that preference for sweet foods was decreased at two months after surgery compared to before, but not at one year. Before surgery, participants had a preference for sweet over savoury taste, $Z=-4.51, p<0.001$. After surgery, participants did not have a preference for either taste; $Z=-1.68, p=0.09$ and $Z=-1.71, p=0.09$ at two months and one year after surgery respectively.

\section{Liking}

Mean liking scores for the macronutrient categories are shown in Figure 5.3. Liking of highcarbohydrate, high-fat and low-energy foods changed over time, all $p<0.05$. Bonferroni-corrected post-hoc analyses showed that the high-carbohydrate and high-fat foods were liked less at two months and one year after surgery, all $p<0.05$. In addition, they indicated increased liking of the low-energy foods one year after surgery, $p=0.05$. Similar to the rankings, liking of high-protein foods did not change over time, $p>0.05$.

Before surgery, liking of high-carbohydrate and high-fat foods was lower than liking of lowenergy foods. High-protein foods were liked more than high-carbohydrate foods, all $p<0.05$. Two months and one year after surgery, low-energy foods were liked more than all other foods. High- 
protein foods were liked more than high-carbohydrate and high-fat foods. Liking for the latter two did not differ.

Mean liking scores for the taste categories are shown in Figure 5.3. Similar to the preference scores, liking of sweet foods differed over time, $p<0.05$. At two months and one year after surgery, participants indicated that they liked the sweet foods less than before surgery, $p<0.05$. Liking of savoury foods did not change over time, $p>0.05$. Before, two months after and one year after RYGB surgery, sweet foods were liked more than savoury foods, all $p>0.05$.

\section{A. Carbohydrate}

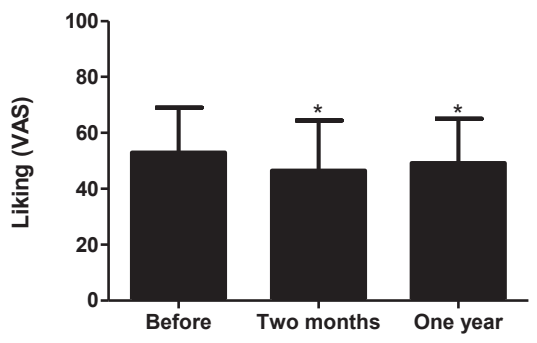

C. Protein

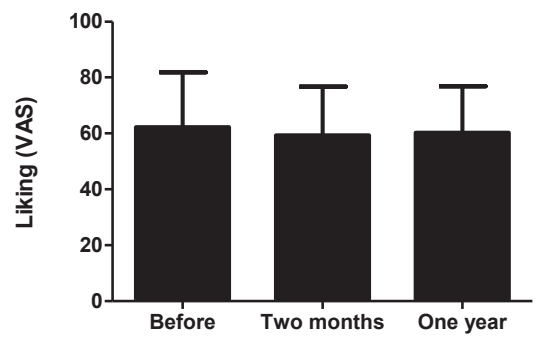

E. Sweet

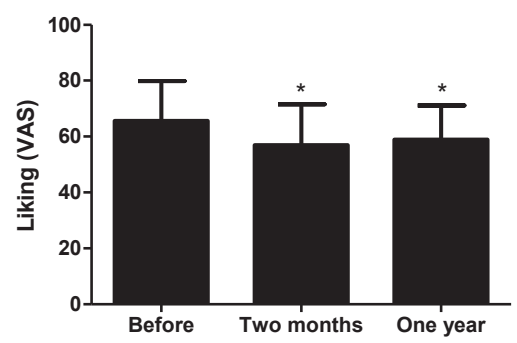

B. Fat

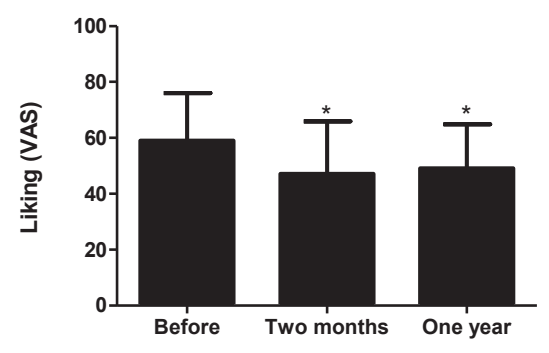

D. Low-energy

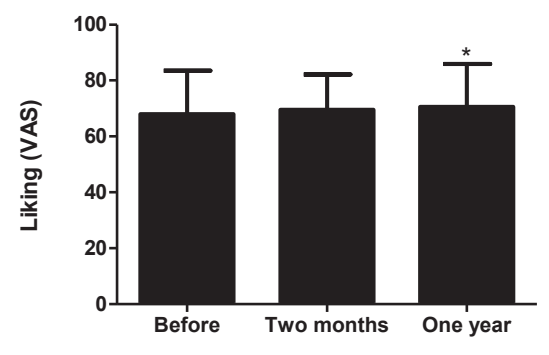

F. Savoury

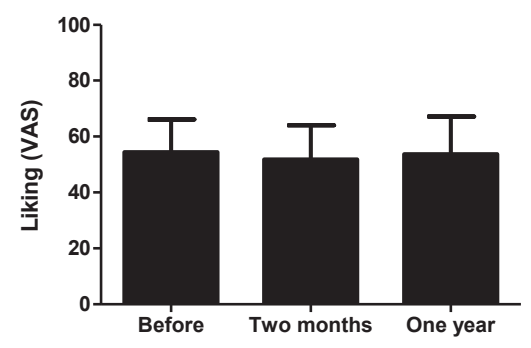

Figure 5.3. Average liking of high-carbohydrate (A), high-fat (B), high-protein (C), low-energy (D), sweet (E) and savoury (F) foods before, two months after and one year after RYGB surgery. Means $\pm S D$ * indicates significant change compared to before surgery at $\mathrm{p}<0.05$ level. 


\section{Correlations}

Loss in BMI over one year was positively correlated with a decrease in preference for highcarbohydrate foods over one year, $\mathrm{r}=0.605, p<0.05$, see Figure 5.4. Loss in BMI did not correlate with changes in preference for foods from other macronutrient or taste categories.

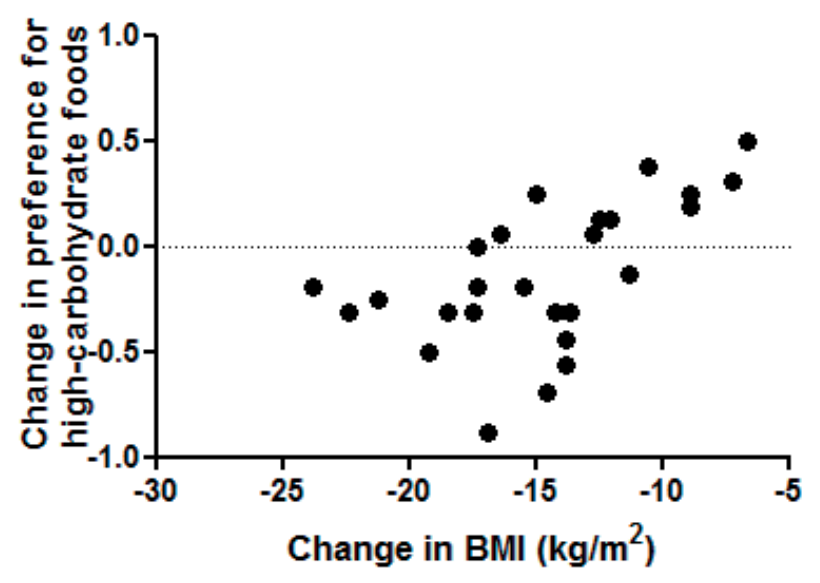

Figure 5.4. Correlation between change in BMI and change in preference for high-carbohydrate foods over one year after RYGB surgery. $\mathrm{R}=0.605, p<0.05$.

\section{Discussion}

The current study assessed changes in food preferences two months and one year after compared with before RYGB surgery. Preference for high-carbohydrate and high-fat foods decreased after surgery, whereas preference for low-energy foods increased. Preference for highprotein foods did not change over time. At two months, but not one year, after surgery, preference for sweet foods was decreased compared with before RYGB.

In line with reported food intake (Mathes and Spector, 2012; Meillon et al., 2013), preference for high-carbohydrate and high-fat foods decreased after surgery. Moreover, the increased preference for low-energy foods is in line with reported increased intake of fruits and vegetables (Laurenius et al., 2013; Ullrich et al., 2013). Preference for high-protein foods did not change from before to after RYGB surgery. As protein intake is tightly regulated (Griffioen-Roose et al., 2012b), preference for protein is less susceptible to change than preference for the other macronutrient categories. Moreover, the participants in the current study received dietary advice on how to obtain sufficient protein intake, which makes it likely that they had a sufficient protein status throughout the study.

In the MTPRT, rankings of food products from different macronutrient and taste categories are used to calculate preference scores. To gain more insight into the meaning of these preference scores, liking ratings can be used. The changes in the liking ratings suggest that the decreased preference (lower ranking) for high-carbohydrate and high-fat foods, and increased preference for low-energy products are caused by decreased liking of high-carbohydrate and high-fat foods, and not by increased liking of low-energy foods. Previous studies showed similar results on liking data, 
that is, decreased liking of high-energy foods and unchanged liking of low-energy foods after RYGB surgery (Faulconbridge et al., 2016; Ochner et al., 2012b). In line with this, a previous study showed decreased reward value of a high-energy food and unchanged reward value of a low-energy food after RYGB surgery (Miras et al., 2012).

The mechanisms behind the changes in food preferences remain to be determined. Previous studies showed lower neural responsivity to high-energy foods after compared with before RYGB surgery (Hansen et al., 2016). The neural changes correlate with changes in desire to eat high-energy foods (Ochner et al., 2012b). Another important change that has been suggested to occur after RYGB is changes in appetite-related gut hormones. A rise in gut hormone concentrations after RYGB surgery might contribute to an increased sensation of satiety (Borg et al., 2006; Meek et al., 2016; Pedersen, 2013). Furthermore, changes in fasting ghrelin concentrations were found to be related to changes in neural responsivity to high-energy foods (Faulconbridge et al., 2016). However, more studies in larger populations are necessary to be able to draw more definitive conclusions.

Another reason for changed food preferences is negative consequences of intake of highenergy foods after RYG surgery. It is estimated that $75 \%$ of patients experiences dumping syndrome to some extent after RYGB surgery, characterized by sweating, palpitation, dizziness and nausea in response to foods high in sugar or fat (Hammer, 2012; Li-Ling and Irving, 2001). Some studie suggest that dumping syndrome decreases preferences for high-energy foods (Schultes et al., 2010; Sugerman et al., 1987). It would be interesting for future studies to investigate the effects of dumping on changes in food preference and liking.

Prior to surgery, high-carbohydrate foods were preferred less relative to foods from the other macronutrient categories. As for liking, high-carbohydrate foods were liked less than highprotein and low-energy foods. This difference in liking might have caused the dispreference for high-carbohydrate foods, although high-fat foods were also liked less than the high-protein and low-energy foods but were not ranked lower. In a previous study that used the MTPRT, highcarbohydrate foods were also liked and preferred less than the other macronutrient categories in a healthy population (de Vries et al., 2017). Further studies are needed to gain insight into the reasons for lower preference for and liking of the high-carbohydrate foods.

The current study was performed on-line. This way patients could easily participate from home at the time of their convenience, instead of having to travel to a test location or inviting researchers to their house for a fifteen-minute test session. However, this gain of convenience led to loss of control over the circumstances under which participants completed the MTPRT. Future on-line studies could take this into account by including a few questions about time since last meal and details on the location and social context.

It is important to note that the rankings in the current study are subject to positive bias, as participants might have had a tendency to give socially desirable answers. These participants are encouraged to lose weight prior to surgery, which makes the low-energy foods the "better" foods. In addition, these patients commonly have a long history of non-surgical weight loss attempts. We would therefore expect that socially desirable answers in the sense that low-energy foods are "liked" and "preferred" are given to the same extent before as after surgery.

The ranking procedure employed in the current study provides more insight into food preferences, as it captures motivation to eat the higher ranked foods over the others. Food choice is 
more than just a comparison of what product is liked more, and liking only predicts food choice to a certain extent. However, it remains to be determined to what extent food choice in the MTPRT predicts actual food choice. Thus far, one study employed an ad libitum buffet meal test in a gastric bypass population. They showed no differences in food choice in the buffet, but an increased preference for low-fat savoury foods in a picture display test (Nielsen et al., 2017). As results of the picture display task were not reported for the separate categories, it is unknown whether this effect is driven by higher preference for low-fat or for savoury foods. The food products in the buffet and in the picture display test were divided over high- and low-fat and sweet and savoury, but the study does not provide insight into energy density (Nielsen et al., 2017), which could explain why no differences in food choice in the buffet task were found.

Even though the current study is one of the few studies to assess (changes in) food preferences over a longer period of time, an increased follow-up time would be interesting. Given that weight regain starts at eighteen to twenty-four months (Courcoulas et al., 2013; Magro et al., 2008), future studies should include follow-up at two years after surgery, to assess whether food preferences remain constant after surgery or return to pre-surgery status.

To conclude, RYGB induces decreased preference for high-carbohydrate and high-fat foods, and increased preference for low-energy foods at two months and one year after surgery. These changes seem to be related to decreased liking of high-carbohydrate and high-fat foods. The change in preference for foods high in carbohydrates is significantly correlated with loss of BMI. 


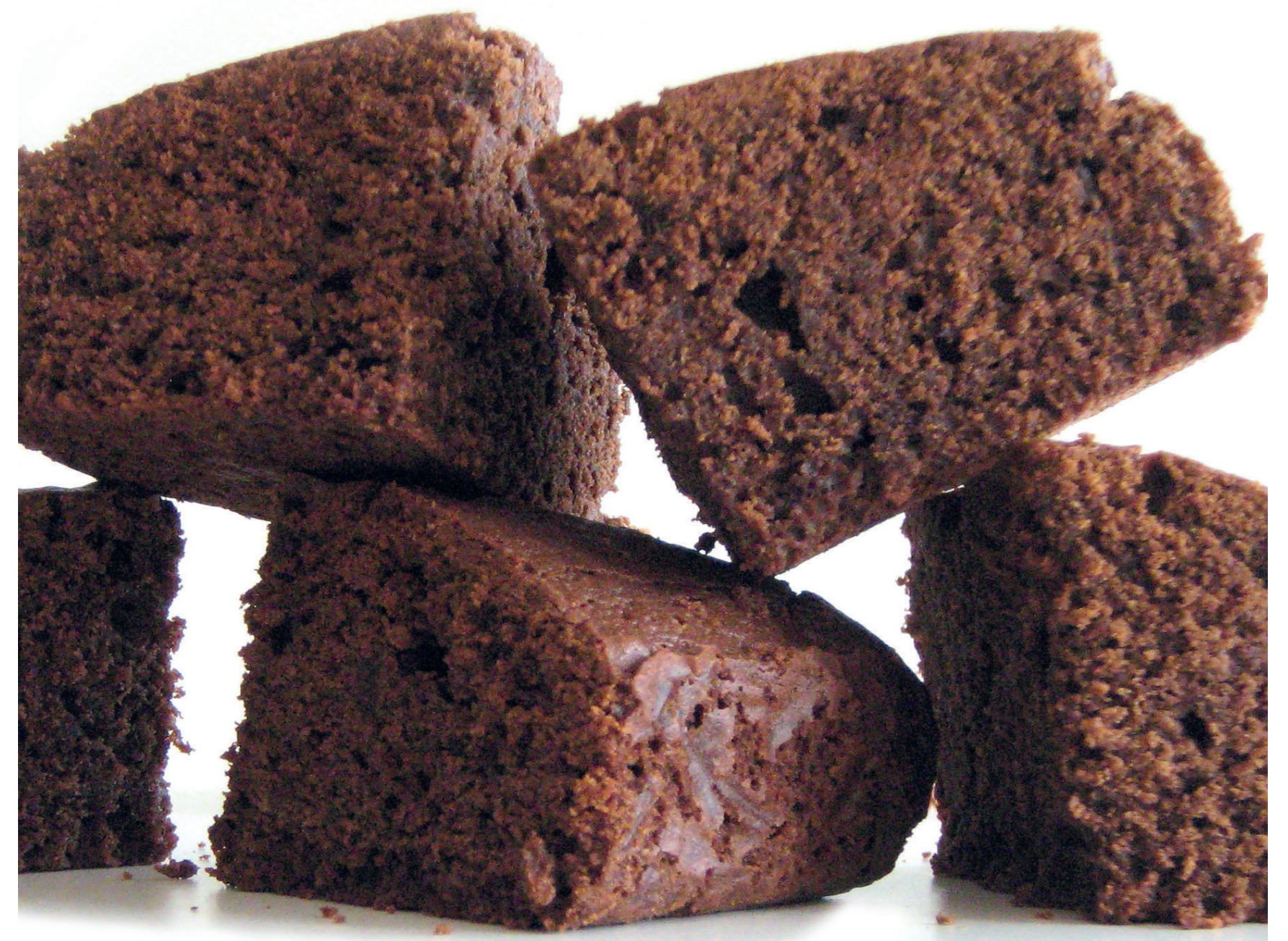




\section{CHAPTER 6}

Altered neURAL ReSPonsivity to FOOd CUES IN ReLATION tO FOOd PREFERENCES, BUT NOT APPETITE-RELATED HORMONE CONCENTRATIONS AFTER RYGB SURGERY

Harriët F.A. Zoon*

Suzanne E.M de Bruijn*

Paul A.M. Smeets

Cees de Graaf

Ignace M.C. Janssen

Wendy Schijns

Edo 0. Aarts

Gerry Jager

Sanne Boesveldt

*Both authors contributed equally 


\begin{abstract}
BACKGROUND: After Roux-en-Y gastric bypass (RYGB) surgery, patients report a shift in food preferences away from high-energy foods.

OBJECTIVE: We aimed to elucidate the potential mechanisms underlying this shift in food preferences by assessing changes in neural responses to food pictures and odours before and after RYGB. Additionally, we investigated whether altered neural responsivity was associated with changes in plasma endocannabinoid and ghrelin concentrations.

DESIGN: 19 RYGB patients (4 men; age $41 \pm 10$ years; BMI $41 \pm 1 \mathrm{~kg} / \mathrm{m}^{2}$ before; BMI $36 \pm 1 \mathrm{~kg} / \mathrm{m}^{2}$ after) participated in this study. Before and two months after RYGB surgery, they rated their food preferences using the Macronutrient and Taste Preference Ranking Task and BOLD fMRI responses towards pictures and odours of high-, and low-energy foods and non-food items were measured. Blood samples were taken to determine plasma endocannabinoid and ghrelin concentrations preand post-surgery.

RESULTS: Patients demonstrated a shift in food preferences away from high-fat/sweet and towards low-energy/savoury food products, which correlated with decreased superior parietal lobule responsivity to high-energy food odour and a reduced difference in precuneus responsivity to highenergy versus low-energy food pictures. In the anteroventral prefrontal cortex (superior frontal gyrus) the difference in deactivation towards high-energy versus non-food odours reduced. The precuneus was less deactivated in response to all cues. Plasma concentrations of anandamide were higher after surgery, while plasma concentrations of other endocannabinoids and ghrelin did not change. Alterations in appetite-related hormone concentrations did not correlate with changes in neural responsivity.
\end{abstract}

CONCLUSIONS: RYGB leads to changed responsivity of the frontoparietal control network that orchestrates top-down control to high-energy food compared to low-energy food and non-food cues, rather than in reward related brain regions, in a satiated state. Together with correlations with the shift in food preference from high- to low-energy foods this indicates a possible role in new food preference formation. 


\section{Introduction}

Roux-en-Y gastric bypass (RYGB) surgery is currently the most effective long-term treatment for morbid obesity (Sjöström, 2013). After RYGB, patients report decreased hunger and lower caloric intake. In addition, a shift in food preferences from high- to low-energy foods is typically observed (Hansen et al., 2016). This change in food preferences has been related to alterations in taste perception and food reward, with fMRI studies showing decreased activation in the mesocorticolimbic reward network in response to high-energy compared to low-energy food cues (Behary and Miras, 2015). However, the exact mechanism behind this shift in food preferences and related neural responses is not completely understood. Potential mediators include changes in subjective hedonic evaluation of food (cues), changes in gut hormones signalling hunger and satiety, post-ingestive side effects of surgery, and changes in nutrient sensing in the gut (Meillon et al., 2013). A better understanding of the mechanisms behind changed food preferences may help to identify factors responsible for the success of this weight-loss intervention, and might guide novel non-surgical strategies that have less risk of complications.

As previously shown, the decreased desire to eat high-energy foods correlates with decreased activation in reward-related brain areas to pictures of high-energy compared to lowenergy foods (Behary and Miras, 2015; Hansen et al., 2016; Ochner et al., 2012b). In a scanner setting it is difficult to realistically mimic an eating environment and thus far, neuroimaging studies used pictures as food cue. However, other sensory modalities might be equally or more important for food choice and the anticipation of food intake. Specifically, food odours play a crucial role in initiating food intake (Bragulat et al., 2010; McCrickerd and Forde, 2016; Yeomans, 2006) by steering appetite (Zoon et al., 2016) and cravings for specific foods (Fedoroff et al., 2003; Larsen et al., 2012). Given their largely unconscious role in priming eating decisions, it is of interest to investigate (alterations in) neural reward responses to palatable food odours as well, in relation to changes in food preferences after RYGB.

There is mounting evidence that gut hormones play a role in altered food preferences after RYGB (Pedersen, 2013). In general, gut hormones play an important role in food choice and food intake, by signalling nutritional status and food reward value to the brain (Bewick, 2012). Most gut hormones are anorexigenic, with higher circulating plasma concentrations resulting in a suppression of food intake. Ghrelin, however, is an orexigenic gut hormone that can stimulate food intake (Wren et al., 2000). Moreover, ghrelin is not only involved in regulating homeostatic eating; eating to fill a need for energy or nutrients, but also in hedonic eating; eating for pleasure (Buss et al., 2014; Monteleone et al., 2012). The orexigenic effect of ghrelin appears to be mediated through the endocannabinoid (eCB) system (Kola et al., 2008). The eCB system is a neuromodulatory system that consists of endogenous ligands, so called endocannabinoids, their receptors and enzymes involved in their synthesis and breakdown. Ghrelin and eCB plasma concentrations increase in anticipation of hedonic eating (Monteleone et al., 2012; Rigamonti et al., 2015), and neuroimaging studies suggest a role for ghrelin and the eCB system in reward processing (Horder et al., 2010; Kroemer et al., 2013; Tudge et al., 2015). Thus far, only a few studies examined the role of ghrelin, and none have explored the eCB system, in relation to altered brain reward activation after RYGB and food preferences (Faulconbridge et al., 2016; Scholtz et al., 2014). 
To improve our understanding of the changes in food preferences typically seen after RYGB, we assessed neural changes in response to high- and low-energy appetizing food cues in two different sensory modalities: odours and pictures. Additionally, to better explain these neural mechanisms, plasma eCB and ghrelin concentrations, as well as food preferences were measured and correlated to changes in brain activation pre- to post-surgery.

\section{Participants and Methods}

\section{Participants}

Twenty-one morbidly obese individuals participated in this study. All participants were enlisted to undergo RYGB surgery at Rijnstate hospital, Arnhem, the Netherlands. Requirements for the surgery were: Body Mass Index (BMI) of $>40 \mathrm{~kg} / \mathrm{m} 2$ or $>35 \mathrm{~kg} / \mathrm{m} 2$ with co-morbidity that was expected to improve after surgically-induced weight loss, long-lasting obesity ( $>5$ years), proven failed attempts to lose weight in a conventional way, intention to adhere to a postoperative followup program. Reasons not to consider individuals for surgery were being pregnant or lactating, psychiatric disorders, alcohol or drug dependency, life threatening conditions or being dependent on the care of others. Individuals were screened for participation in the study at Rijnstate hospital. All participants were right-handed, non-smoking, did not have conditions that interfered with the MRI measurements (e.g. claustrophobic, metal implants, pacemaker, neurological disorders), had a normal sense of smell (scoring $\geq 10$ on the identification part of the Sniffin' Sticks; Hummel et al., 2007), were not vegetarian and did not have allergies or intolerances to the foods used and cued (visual/olfactory stimuli) in the study. Participants received a monetary reward for their contribution. All participants provided written informed consent before entering the study. The protocol was approved by the Medical Ethical Committee of Wageningen University (NL45837.081.13) and was executed in accordance with the ethical principles of the Declaration of Helsinki of 1975, as revised in 2013.

\section{Overall design and experimental procedure}

This study had a $2 \times 2 \times 3$ within-subject design, including the factors time (pre- and postgastric bypass surgery), stimulus modality (visual/olfactory) and stimulus category (high-fat/highsugar (HFHS) food, low-fat/low-sugar (LFLS) food, Non-Food (NF)).

Participants visited the test facilities at three occasions. First, they were familiarized with the MRI test environment and the experimental task and stimuli used, in a dummy MRI scanner at Wageningen University (training session). Following the training session, there were two identical test sessions during which the actual measurements were taken. The first test session took place 3.4 ( $\mathrm{SD}=1.8$ ) weeks before and the second test session took place 9.2 ( $\mathrm{SD}=1.3$ ) weeks after RYGB. Each participant was scanned at approximately the same time of day for both sessions, between 14:0017:00 at hospital Gelderse Vallei (Ede, The Netherlands). Participants were instructed to refrain from eating and drinking anything but water and weak tea in the three hours before a test session. Upon arrival at the hospital, blood samples were taken for analysis of plasma endocannabinoid and ghrelin concentrations. In order to measure responses underlying hedonic eating (eating for pleasure, in the absence of hunger), participants were offered orange juice, and after a short break 
they consumed a standardized meal consisting of bread roll(s), cheese, ham and butter (see Supplementary Table 6.1 for more detailed information), to evoke a state of satiety (Table 6.1). Following this, they waited for 15 minutes to allow digestion. Before entering the MRI room, participants were presented with the odours and pictures used in the reward task to familiarize with the stimuli and reinforce the appropriate association with the stimuli. They also rated their hunger, fullness, prospective consumption, desire to eat, and thirst on 100 point visual analogue scales (VAS). During the scan session, first, a reward task was performed while functional MR images were acquired. Second, structural MR images were collected. Thereafter, participants took part in two additional functional runs in which a food-related go/no-go task was performed (data reported elsewhere). At the end of the test session, olfactory performance was assessed using the Sniffin' Sticks (threshold, discrimination, identification; Hummel et al., 2007). The regular Identification 16 was used during screening, thus to prevent a potential learning effect, we used the Identification 16+ for the second test session (Sorokowska et al., 2015). Paired sample T-tests revealed that the overall olfactory performance (TDI score) was not different between test sessions (before: $33.7 \pm 4.7$; after: $35.0 \pm 4.2 ; p=0.328$.

Table 6.1. Weight and hunger ratings pre- and post-surgery.

\begin{tabular}{lccr}
\hline & Pre-surgery & Post-surgery & Significance \\
\hline Weight $(\mathrm{kg})$ & $120 \pm 14$ & $104 \pm 15$ & $p<0.001$ \\
BMI $\left(\mathrm{kg} / \mathrm{m}^{2}\right)$ & $41 \pm 3$ & $36 \pm 4$ & $p<0.001$ \\
\hline Hunger & $13 \pm 21$ & $11 \pm 24$ & $p=0.738$ \\
Fullness & $70 \pm 25$ & $68 \pm 34$ & $p=0.867$ \\
Prospective consumption & $27 \pm 25$ & $8 \pm 18$ & $p=0.020$ \\
Desire to eat & $22 \pm 20$ & $12 \pm 23$ & $p=0.197$ \\
Thirst & $66 \pm 26$ & $51 \pm 30$ & $p=0.022$ \\
\hline
\end{tabular}

Hunger ratings performed on a 100 point VAS. Values are means \pm SD. BMI $=$ Body mass index.

\section{Food preferences}

Food preferences were assessed two weeks before and two months after RYGB using the online version of the macronutrient and taste preference ranking task (MTPRT; de Bruijn et al., 2017). In this task, participants were presented with four pictures of different food products at a time and asked to rank the products according to what they most desire to eat at that moment. Food products included in this task were either high in carbohydrate, high in fat, high in protein or low in energy, and had a sweet or savoury taste. The MTPRT was presented in EyeQuestion software (Logic8 BV).

\section{Food stimuli (odours and pictures)}

Odours and pictures were selected to signal either high-fat, high-sugar food (HFHS), lowfat, low-sugar food (LFLS) or non-food (NF) items (as control) by means of pilot studies in separate samples of participants. Odours were selected to be similar in perceived intensity and liking, different in the associated energy-density, and correctly associated to the corresponding food product/object. The selected odours were Chocolate (HFHS; International Flavors and Fragrances 
(IFF) 10810180; 8.5\% in Propylene Glycol (PG)), Caramel (HFHS; IFF 10895342; 20\% in PG), Tomato (LFLS; IFF 15039016; 24\% in PG), Cucumber (LFLS; IFF 73519595; 34\% in PG), Fresh Green (NF; AllSens-Voit Aroma Factory No. 819; 2.2\% in PG), Wood (NF; AllSens-Voit Aroma Factory No. $821 ; 2.2 \%$ in PG). Pictures were selected to be similar in liking, and consistently matched to a food product/object and to one of the selected odours. For each odour we selected three different pictures to reduce effects of boredom. We selected Chocolate muffin, Brownie, and Chocolate bonbons for Chocolate odour; Caramel ice-cream, Stroopwafel (Dutch caramel syrup waffle), and Boterkoek (Shortbread) for Caramel odour; Tomato slices with pepper, Tomato slices, and Tomato slices with basil for Tomato odour; Cucumber slices with peel, Cucumber salad, and Cucumber chunks for Cucumber odour; Green soap, Tulips, and White flowers for Fresh Green odour; and Chunk of wood, Pine branches, and Purple soap for Wood odour. Standardized food images used in the fMRI task were provided by the Image Sciences Institute, UMC Utrecht, and created as part of the Full4Health project (www.full4health.eu), funded by the European Union Seventh Framework Program (FP7/2007-2013) under grant agreement nr. 266408, and the I.Family project (http://www.ifamilystudy.eu), grant agreement nr. 266044 (Charbonnier et al., 2015b).

\section{(f)MRI paradigm and measurements}

The reward task lasted $\sim 40$ min and consisted of olfactory and visual cues of HFHS food, LFLS food and NF were presented one by one, in pseudo random order (see Figure 6.1). Six olfactory stimuli and 18 visual stimuli (3 related to each odour) were presented. Each odour was presented 15 times and each picture was presented 5 times, resulting in a total of 90 odour and 90 visual presentations. Trials started with the presentation of a red fixation cross (1s) during which participants were instructed to slowly inhale via the nose. Following this, either an odour or a picture (2s) was presented. During presentation of an odour the red fixation cross remained on the screen. Over the entire run participants were asked to provide liking and wanting ratings twice for each stimulus, and intensity ratings for the odours (7s). Between trials a rest period (3-11s) was included, during which a white fixation cross was visible. The inter-stimulus interval between odour presentations was kept between 17-24 s to prevent adaptation and was jittered to prevent habituation. Olfactory stimuli were presented using an fMRI-compatible computer-controlled 8channel olfactometer (Burghart, Wedel, Germany) that delivered the odours via a small nasal cannula in a constant air flow $(8 \mathrm{~L} / \mathrm{min})$ that was heated to $37^{\circ} \mathrm{C}$ and humidified to $80 \%$ relative humidity to prevent irritation of the nasal mucosa. Visual stimuli were projected using a backprojection screen, which could be viewed by the participants via a mirror positioned on the head coil. The reward task ran in E-Prime 2.0 Professional (Psychology Software Tools Inc.). An MR compatible button box was used to answer questions in the task. Head movements were restricted by placing foam cushions next to the participants' head. In addition, surgical tape was placed across the forehead to provide feedback on head movements. Earplugs were provided for noise reduction.

A 3-Tesla Siemens Magnetom Verio MRI scanner in combination with a 32-channel head coil was used, to acquire 993 T2*-weighted gradient echo images with BOLD contrast (repetition time $=2240 \mathrm{~ms}$, echo time $=25 \mathrm{~ms}$, flip angle $=90^{\circ}$, field of view $=192 \times 192 \mathrm{~mm}, 45$ axial slices, ascending order, voxel size $3 \times 3 \times 3 \mathrm{~mm}$ ) in one functional run. The imaging volume was tilted at an oblique angle of $30^{\circ}$ to the anterior-posterior commissure line to reduce signal dropout in the 
orbitofrontal and ventral temporal lobes (Deichmann et al., 2003). A high-resolution T1-weighted anatomical MRI scan was acquired (MPRAGE: repetition time $=1900 \mathrm{~ms}$, echo time $=2.26 \mathrm{~ms}$, flip angle $=9^{\circ}$, field of view $=256 \times 256 \mathrm{~mm}, 192$ sagittal slices, voxel size $=0.5 \times 0.5 \times 1 \mathrm{~mm}$ ).

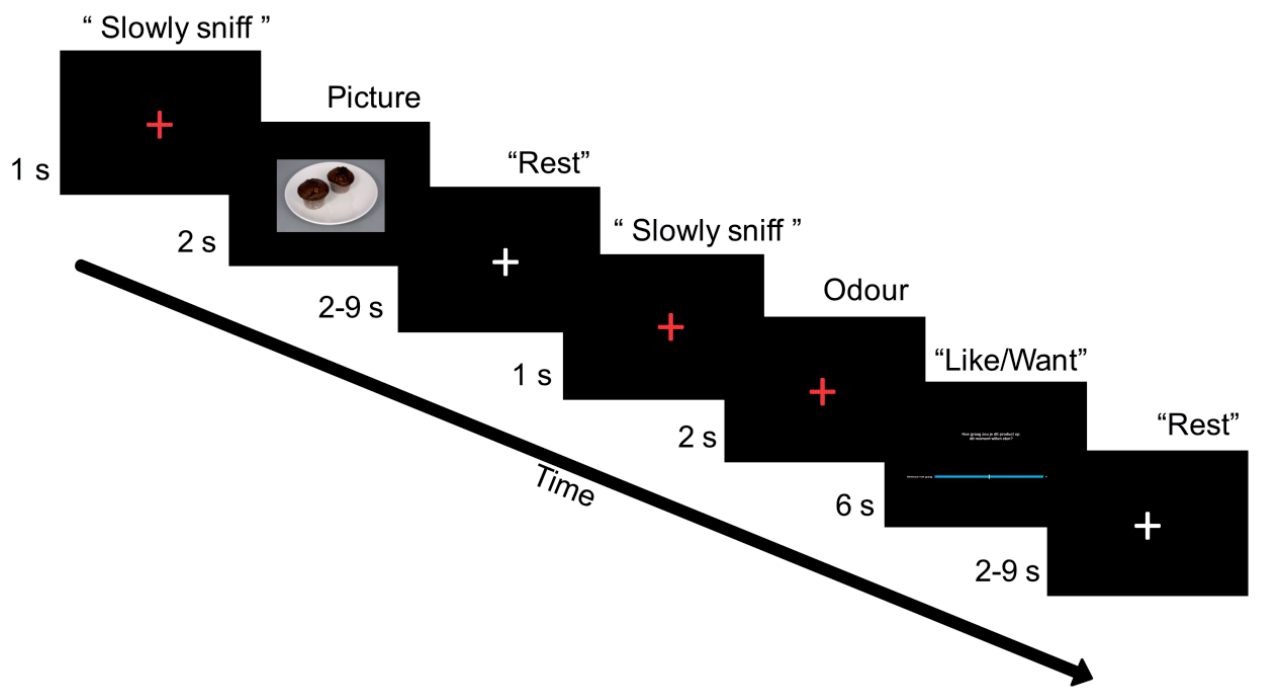

Figure 6.1. Design of the fMRI reward paradigm. Trial presentation started with a red fixation cross for 1s during which participants were instructed to slowly inhale via the nose ('slowly sniff), an odour or picture was then presented for 2s. Occasionally participants provided liking, wanting or intensity ratings on a 100 point VAS within 7s. Between trials a rest period (3-11s) was included, during which a white fixation cross was visible.

\section{Data analyses}

Results are expressed as means \pm SD unless otherwise specified. Behavioural data were analysed in SPSS Statistics for Windows, Version 22.0 (IBM Corp., Armonk, NY, USA). Results were considered statistically significant at $\mathrm{p}<0.05$. Paired-samples $\mathrm{T}$-tests were used to test differences in weight, BMI and hunger ratings pre- and post-surgery. Mixed-models were used to analyse the differences in liking, wanting and intensity ratings that were provided in the test sessions before surgery and after surgery (see Supplementary Table 6.2).

Food preferences. Sixteen participants completed the online MTPRT pre- and post-surgery. Preference scores for high-fat sweet and low-energy savoury products were calculated based on rankings in the MTPRT using the formula below. The higher the rank, the higher the score. The preference scores can range from 1 to 4 (de Bruijn et al., 2017).

$$
\text { preference score }=\frac{4 *(\# \operatorname{rank} 1)+3 *(\# \operatorname{rank} 2)+2 *(\# \operatorname{rank} 3)+1 *(\# \operatorname{rank} 4)}{8}
$$

Paired samples T-test were used to compare preference scores for high-fat sweet and lowenergy savoury pre- and post-surgery.

Ghrelin and endocannabinoids. Blood was collected in tubes with EDTA as anticoagulant. To one tube, 4-(2-aminoethyl)benzenesulfonyl fluoride hydrochloride (Sigma-Aldrich) was added to reach a concentration of $1 \mathrm{mg} / \mathrm{ml}$ in the collected blood. The tubes were centrifuged at $1300 \mathrm{xg}$ 
for 10 minutes at $4{ }^{\circ} \mathrm{C}$. Plasma was then portioned into aliquots and stored at $-80^{\circ} \mathrm{C}$. Prior to storage, hydrochloric acid was added to a final concentration of $0.05 \mathrm{~N}$ to the plasma that contained 4-(2aminoethyl)benzenesulfonyl fluoride hydrochloride. This plasma was later used for ghrelin analyses. To another aliquot, phenylmethylsulfonyl fluoride (Sigma-Aldrich) and URB602 (SigmaAldrich) were added to a final concentration of $100 \mu \mathrm{M}$ for both. This was used to measure plasma concentrations of the eCBs anandamide and 2-arachidonoylglycerol (2-AG), and the related Nacylethanolamines docosahexaenoyl ethanolamide (DHEA), dihomo- $\gamma$-linoleonylethanolamide (DLE), oleoylethanolamide (OEA), palmitoylethanolamide (PEA), stearoylethanolamide (SEA), using an LC-MS/MS technique described elsewhere (Balvers et al., 2009). ELISA was used to measure total ghrelin concentrations in plasma (Millipore).

For each biochemical parameter, normality was checked. Only DHEA was normally distributed and a paired-samples T-test was used to compare plasma concentrations before and two months after surgery. For all other parameters Related-Samples Wilcoxon Signed Rank Tests were used. Correlations between pre- to post- surgery changes in gut hormones (eCBs, ghrelin) and changes in BMI, changes in body weight, and changes in liking and wanting of the stimuli were tested in SPSS using Spearman's rho correlation coefficient.

$f M R I$. Two out of 21 datasets were excluded from further analyses, because realignment parameters indicated substantial movement artefacts $(>5 \mathrm{~mm})$. Whole brain functional images of 19 individuals ( 4 men and 15 women; age $41 \pm 10$ years) were preprocessed and analysed using the SPM12 software package (Wellcome Trust Centre for Neuroimaging, London, UK) run with MATLAB 7.12.0 (R2011a, The Mathworks Inc.). Functional images were slice timed, realigned and coregistered. A DARTEL framework was used to create a study-specific template and participantspecific deformation fields (Ashburner, 2007). The images were then spatially normalized to the MNI standard brain using the study-specific DARTEL template and the participant-specific deformation fields. Smoothing was applied to the normalized images using an isotropic Gaussian kernel with a 6-mm full width at half maximum.

Subject level analyses: Each test session (pre-/post-surgery) was modelled separately. Motion related variance was corrected for by including motion-correction parameters in the model. Subject level analyses included calculation of six contrast images for odour, and six contrast images for picture presentations (HFHS vs rest; LFLS vs rest; NF vs rest; HFHS vs LFLS; HFHS vs NF; LFLS vs NF). Image calculation was used to subtract the contrast images post-surgery from the contrast images pre-surgery, creating a contrast image in which the within-subject changes from pre- to post-RYGB were captured.

Group level analyses. For our main contrast of interest, pre- to post-surgery changes in the difference between BOLD responses to HFHS and LFLS cues (HFHS >LFLSpre-HFHS >LFLSpost) were analysed for odours and pictures in two separate T-tests. Six T-tests were performed to analyse pre- to post-surgery differences in BOLD responses to the different stimulus categories (HFHSpreHFHSpost; LFLSpre-LFLSpost; NFpre-NFpost;), for visual and olfactory cues separately. Also, we performed four T-tests to analyse differences in BOLD responses between HFHS and NF stimuli and between LFLS and NF stimuli (HFHS>NFpre-HFHS>NFpost; LFLS >NFpre-LFLS >NFpost), separate for odours and pictures. We used a whole brain approach, with a significance level of $\mathrm{p}=.001$ (unc.) and a cluster extent threshold of $\mathrm{k}=8$ contiguous voxels. For all contrasts, the mean beta values of 
significant clusters were extracted with use of the MarsBar toolbox (http://marsbar.sourceforge.net/). Mean beta values of each significant cluster were subsequently correlated with pre- to post- surgery changes in endocannabinoid and ghrelin concentrations, changes in BMI, changes in body weight, changes in liking and wanting of the stimuli, and changes in preference for high-fat sweet and low-energy savoury products. Correlation analyses were performed in SPSS using Spearman's rho correlation coefficient.

\section{Results}

After RYGB surgery, the mean body weight of our study population decreased from $120 \pm 3$ to $104 \pm 3 \mathrm{~kg}$, a mean weight loss of $16 \pm 4 \mathrm{~kg}$. This weight change led to a decrease in BMI from $41 \pm 1$ to $36 \pm 1 \mathrm{~kg} / \mathrm{m} 2$ (see Table 6.1).

\section{Hunger, liking and wanting ratings}

Our standardized meal was successful in achieving a state of satiety, as observed from the hunger, fullness and desire to eat ratings, which were similar before and after RYGB surgery. Ratings for prospective consumption and thirst were significantly higher pre- compared to post-surgery (see Table 6.1).

HFHS pictures were significantly less liked (pre: $47 \pm 6$, post: $29 \pm 5$ ) and wanted (pre: $42 \pm 6$, post: $26 \pm 5$ ) after surgery (both $p<0.001$ ). Similarly, HFHS odours were less liked (pre: $50 \pm 8$, post: $30 \pm 7 ; p<0.001$ ) and less wanted (pre: $40 \pm 7$, post: $24 \pm 6 ; p=0.018$ ) after surgery. Liking and wanting ratings remained the same in both test sessions for LFLS pictures and odours, and for NF pictures. NF odours were significantly less liked (pre: $35 \pm 7$; post: $23 \pm 7$; $p=0.008$ ) and wanting ratings were similar pre- and post-surgery. Intensity ratings for HFHS, LFLS and NF odours were similar between the two test sessions (for all, see Supplementary Table 6.2).

\section{Food preferences}

Preference for high-fat/sweet products decreased after surgery (pre: $2.6 \pm 0.72$, post: $2.0 \pm 0.8$; $\mathrm{T}(1,15)=3.39, p<0.05)$. Preference for low-energy/savoury products increased after surgery (pre: $2.3 \pm 0.6$, post: $2.7 \pm 0.6 ; \mathrm{T}(1,15)=-3.50, p<0.05)$.

\section{Functional imaging data}

Pictures: Pre- to post-surgery differences. In the left precuneus, the difference in brain responses to HFHS and LFLS pictures was significantly smaller after compared to before surgery (see Table 6.2 and Figure 6.2). Before surgery, viewing LFLS cues led to greater deactivation of the left precuneus than viewing HFHS cues. After RYGB, deactivation in response to food pictures was minimal in this region and did not differ between HFHS and LFLS. Participants showed significantly smaller responses to LFLS pictures in the left superior frontal gyrus (anteroventral PFC) after surgery (see Table 6.2). The pre-surgical deactivation of this region to LFLS pictures, was no longer present in the post-surgery session. Right superior parietal lobule responses to NF pictures were significantly different pre- and post-RYGB, showing deactivation pre- and activation post-surgery (see Table 6.2). Further, the difference in right precuneus response to HFHS compared to NF pictures was reversed 
from pre- to post-surgery (see Table 6.2 and Figure 6.2). Deactivation in response to HFHS pictures was greater than deactivation to NF pictures before surgery, and smaller than deactivation to NF pictures after surgery.

Table 6.2. Significant differences in neural activation by food and non-food cue exposure (picture or odour), pre- to post-RYGB

\begin{tabular}{|c|c|c|}
\hline cluster size & Z-score & $\begin{array}{c}\text { Peak } \\
\text { coordinates }\end{array}$ \\
\hline & & $y$ \\
\hline
\end{tabular}

\begin{tabular}{|c|c|c|c|c|c|c|c|}
\hline PICTURE & & & & & & & \\
\hline $\begin{array}{l}\text { HFHS-LFLS } \\
\text { HFHe }>\end{array}$ & $\mathrm{L}$ & Precuneus & 9 & 3.43 & -3 & -54 & 69 \\
\hline LFLS $_{\text {pre }}<$ LFLS post & $\mathrm{L}$ & $\begin{array}{l}\text { Superior Frontal } \\
\text { gyrus/anteroventral PFC }\end{array}$ & 13 & 3.73 & -21 & 51 & 9 \\
\hline $\mathrm{NF}_{\text {pre }}<\mathrm{NF}_{\text {post }}$ & $\mathrm{R}$ & Superior Parietal lobule & 11 & 3.45 & 24 & -66 & 63 \\
\hline $\begin{array}{l}\text { HFHS-NF } \text { pre }<\text { HFHS- } \\
\text { NF }_{\text {post }}\end{array}$ & $\mathrm{R}$ & Precuneus & 8 & 3.49 & 15 & -54 & 21 \\
\hline ODOUR & & & & & & & \\
\hline HFHS $_{\text {pre }}<$ HFHS $_{\text {post }}$ & $\mathrm{R}$ & Precuneus & 9 & 3.92 & 15 & -42 & 51 \\
\hline & $\mathrm{L}$ & Superior Parietal lobule & 8 & 3.66 & -21 & -54 & 51 \\
\hline LFLS $_{\text {pre }}<$ LFLS $_{\text {post }}$ & $\mathrm{R}$ & Precuneus & 11 & 3.57 & 9 & -48 & 72 \\
\hline & $\mathrm{R}$ & Superior Parietal lobule & & 3.52 & 15 & -54 & 72 \\
\hline & $\mathrm{L}$ & Precuneus & 10 & 3.51 & -3 & -66 & 45 \\
\hline $\mathrm{NF}_{\text {pre }}<\mathrm{NF}_{\text {post }}$ & $\mathrm{L}$ & Superior Parietal lobule & 20 & 3.89 & -21 & -54 & 69 \\
\hline & L & Precuneus & 12 & 3.69 & -12 & -69 & 63 \\
\hline $\begin{array}{l}\text { HFHS-NF } \mathrm{Nre}>\mathrm{HFHS}- \\
\mathrm{NF}_{\text {post }}\end{array}$ & L & $\begin{array}{l}\text { Superior Frontal gyrus / } \\
\text { anteroventral PFC }\end{array}$ & 8 & 3.92 & -21 & 63 & 3 \\
\hline
\end{tabular}



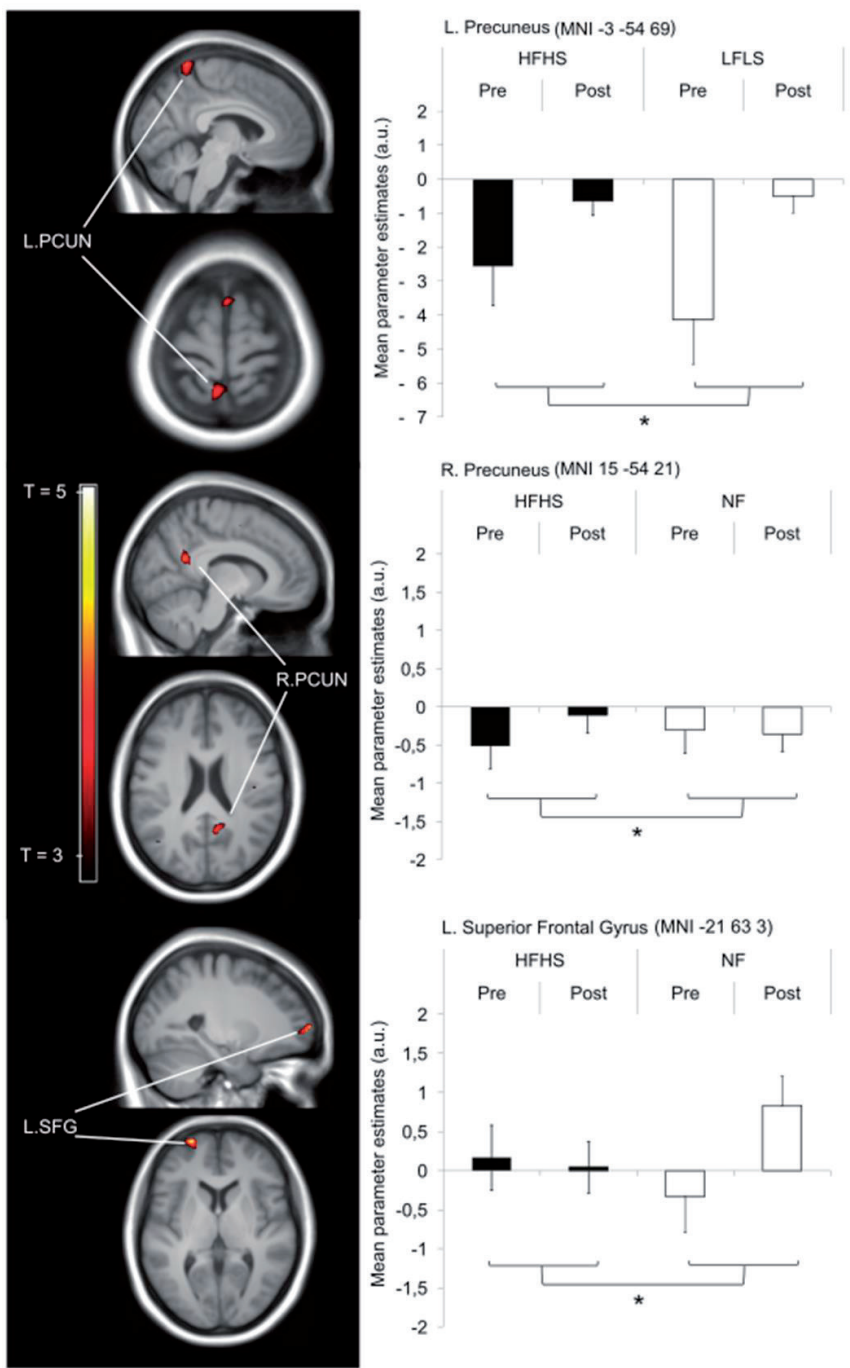

Figure 6.2. Significant differences in neural activation by food and non-food cue exposure (picture or odour), pre- to post-RYGB. Results for the brain images were thresholded at $\mathrm{p}=.005$ for visualization. Upper. Post-surgery, the left precuneus (L.PCUN, MNI: -3 -54 69) showed significantly less difference between activation to low-fat/low-sugar (LFLS) and high-fat/high-sugar (HFHS) food pictures ( $k=9, \mathrm{z}=3.43$ ). Pre-surgery, more deactivation was observed in response to LFLS compared to high-fat/high-sugar (HFHS) pictures while post-surgical responses to these two stimuli appeared similar. Middle: Post-surgery, the right precuneus (R.PCUN, MNI: 15 -54 21) showed significantly more difference between activation to high-fat/high-sugar (HFHS) food and non-food (NF) pictures ( $k=8, \mathrm{z}=3.49)$. Pre-surgical deactivation in response to HFHS food pictures was higher, while post-surgical deactivation to HFHS food pictures was lower compared to nonfood pictures. Lower. Post-surgery, the left superior frontal gyrus (L.SFG, MNI: -21 63 3) showed significantly less difference between activation to high-fat/high-sugar (HFHS) food and non-food (NF) odours ( $\mathrm{k}=8, \mathrm{z}=3.92)$. Post- compared to pre-surgery, activation to HFHS odour appeared similar, while increased activation was observed in response to NF odour. 
Odours: Pre- to post-surgery differences During HFHS odour exposure significantly greater deactivation was found in the right precuneus and left superior parietal lobule pre- compared to post-surgery (see Table 6.2). We also observed significantly different precuneus responses to LFLS odours pre- versus post-surgery (see Table 6.2). The right precuneus was deactivated pre- and activated post-surgery. Before surgery there was an absence of response in the left precuneus, but after surgery this region was activated in response to LFLS odours. In response to NF odours deactivation of the left precuneus and left superior parietal lobule was observed pre- surgery, this was significantly different after surgery, when activation was seen in these regions (see Table 6.2). The difference in response of the left superior frontal gyrus (anteroventral PFC) to HFHS odours vs NF odours was significantly different pre- and post-surgery (see Table 6.2 and Figure 6.2). This effect appears to be mainly driven by increased activation in response to NF odours after surgery.

\section{Correlations between neural changes and behavioural measures}

No significant correlations were found between changes in neural responses to odours or pictures, changes in BMI or body weight, and changes in liking, wanting and intensity ratings for odours (all $p>0.05$ ). An increased reduction of deactivation of the left superior parietal lobule to HFHS odour after RYGB was correlated with a greater increase in preference for low-fat/savoury food ( $\mathrm{r}=-0.550, p=0.027$ ), and albeit not significant, associated with a greater decrease in preference for high-fat/sweet food ( $\mathrm{r}=0.417, p=0.108)$. An increased reduction in activation of the left precuneus to HFHS pictures relative to LFLS pictures was correlated with a greater decrease in preference for high-energy/sweet relative to low-energy/savoury food ( $\mathrm{r}=-0.530, p=0.035)$.

\section{Endocannabinoids and ghrelin}

Plasma concentrations of anandamide were significantly greater post- than pre-surgery (before: $0.38 \pm 0.17 \mathrm{ng} / \mathrm{ml}$, after: $0.48 \pm 0.15 \mathrm{ng} / \mathrm{ml} . \mathrm{Z}=-2.77, p=0.006$. For the other eCBs, $2-A G$, DHEA, DLE, OEA, PEA, SEA, and ghrelin, concentrations did not change significantly (see Table 6.3 for values).

Changes in $\mathrm{eCB}$ and ghrelin concentrations did not correlate with changes in neural activation, nor with changes in liking and wanting of pictures and odours, nor with BMI or body weight changes (all $p>0.05$ ). 
Table 6.3. Plasma concentrations of endocannabinoids and ghrelin pre- and post-RYGB surgery

\begin{tabular}{lccc}
\hline Compound & Pre-surgery & Post-surgery & Difference \\
\hline Anandamide* & $0.4 \pm 0.2$ & $0.5 \pm 0.2$ & $0.1 \pm 0.2$ \\
2-arachidonoylglycerol & $8 \pm 3$ & $14 \pm 17$ & $6 \pm 17$ \\
Docosahexaenoylethanolamide & $0.5 \pm 0.1$ & $0.4 \pm 0.1$ & $-0.1 \pm 0.2$ \\
Dihomo- $\gamma$-linoleonylethanolamide & $0.1 \pm 0.05$ & $0.09 \pm 0.04$ & $-0.01 \pm 0.05$ \\
Oleoylethanolamide & $2.6 \pm 1$ & $2.6 \pm 1$ & $0.0 \pm 1$ \\
Palmitoylethanolamide & $1.7 \pm 0.7$ & $1.6 \pm 0.4$ & $-0.17 \pm 0.8$ \\
Stearoylethanolamide & $2.5 \pm 1$ & $2.2 \pm 1$ & $-0.3 \pm 1$ \\
Ghrelin & $459 \pm 190$ & $442 \pm 171$ & $-17 \pm 159$ \\
\hline
\end{tabular}

Concentrations in $\mathrm{ng} / \mathrm{mL}$. Values are means $\pm \mathrm{SD}$. $\mathrm{n}=18$, for endocannabinoids, $\mathrm{n}=17$ for ghrelin. ${ }^{*}$ Significant difference $(p=0.006)$ pre- and post-Roux-en-Y gastric bypass (RYGB).

\section{Discussion} underlying changes in food preferences after RYGB, using appetizing visual and olfactory cues representing high- and low-energy foods, and by including measures of appetite-related hormones (eCBs and ghrelin). After RYGB, patients demonstrated a shift in food preferences away from highfat/sweet and towards low-energy/savoury food products, which correlated with less deactivation of the superior parietal lobule to high-energy food odours and a smaller difference in activation of the precuneus to high-energy versus low-energy food pictures. Main findings further included less deactivation of the precuneus in response to all cues. After surgery, deactivation in the superior frontal gyrus was less towards low-energy food pictures and more similar to high-energy versus non-food odours. Further, anandamide concentrations were increased, while other eCB and ghrelin concentrations did not change. Neural changes did not correlate with changes in eCB and ghrelin concentrations.

In line with previous research (Hansen, 2014), participants reported a shift in food preferences away from high-energy foods, and towards low-energy food products, on the MTPRT. These alterations in food preferences may (in part) underlie the success of RYGB surgery as weightloss intervention.

Post- compared to pre-surgery, the left superior frontal gyrus was less deactivated in response to low-, but not to high-energy pictures. Previous research into the effects of RYGB reported postsurgical reductions in prefrontal cortex activation that were more pronounced for high- relative to low-energy cues (Geliebter, 2013). Moreover, decreased differences in desire to eat high- versus low-energy foods were predicted by a reduced difference in a more posterior part of the superior frontal gyrus response to high-energy versus low-energy food cues (Ochner et al., 2012b). Unfortunately, these studies only compared surgery-related changes in responses to highrelative to low-energy food cues, rather than considering changes in responses to high and lowenergy food cues separately. Perhaps, the decreased difference in activation between high- and lowenergy food cues are in fact largely driven by a diminished response to low-energy food cues, similar to our observations. The anteroventral region of the superior frontal gyrus we found has been implicated in subjective reward value (Gross et al., 2014) and in processes of self-control (Diekhof 
et al., 2012). Several studies have reported that activation patterns in this region can act as a predictor of food choices (Gross et al., 2014; Schonberg et al., 2014; Van der Laan et al., 2012). Changes in superior frontal activation could be related to the beneficial shift in food preferences, from high-energy towards more healthy low-energy foods, that is frequently reported after RYGB surgery (Behary and Miras, 2015; Berthoud et al., 2012; Ochner et al., 2011; Thirlby et al., 2006) and is also confirmed by the current study.

Here we have extended previous research into RYGB by including olfactory food cues that predict the immediate presence of food and the rewarding effects of eating (Bragulat et al., 2010) and are processed largely unconsciously (Shepherd, 2006). After surgery, we observed less deactivation of the superior parietal cortex, in response to food odours but not pictures. Increased superior parietal activation was previously found during anticipation of reward (Krebs et al., 2012). This region was also proposed to be part of a top-down control system for attentional processes that is modulated by implicit contextual cues, with greater deactivation being associated with increased attentional demands (King et al., 2012; Krebs et al., 2012). Less deactivation of the superior parietal cortex after surgery found in this study could indicate a decrease in anticipated reward of highenergy food odours, and thereby a lowered attentional demand. In line with this, the diminished activation was positively, albeit not significantly, correlated with (lowered) preference for highenergy foods, and negatively correlated with (heightened) preference for low-energy foods.

After surgery, decreased activation of the bilateral precuneus was found in response to high, but most pronounced to low-energy dense food cues (pictures and odours). In previous research, decreased precuneus responsivity to high- versus low-energy food cues was related to decreased food liking (Ochner et al., 2012b). A role in food reward anticipation has been proposed by other studies (Bjork and Hommer, 2007; Filbey et al., 2012; King et al., 2012; Stice et al., 2011; Stice et al., 2012; Stoppel et al., 2011; Takemura et al., 2011; van Duijvenvoorde et al., 2014; Wittmann et al., 2005). Alternatively, the difference in precuneus activation before and after surgery could be related to changes in attentional control (Chen et al., 2010; King et al., 2012). Greater deactivation in this region indicates increased attentional demand. In relation to our data this would mean that food cues in general, but low-energy food cues in particular, recruit less attention after surgery. Further research should focus in more detail on how (changes in) attentional processes relate to changes in food preference and choice rather than food cue reactivity.

The neural regions (the superior frontal gyrus, superior parietal lobe and precuneus) in which we find altered responsivity to food cues are all part of a frontoparietal control network involved in adaptive top-down control (Schonberg et al., 2014; van den Heuvel et al., 2013). In a study by Schonberg et al. (Schonberg et al., 2014) participants who were trained to choose less preferred food item showed decreased activation in this frontoparietal network during low-value food choices. The authors propose that over the course of extensive training the need for top-down frontoparietal control reduces as food preference responses move from goal-directed to more habitual behaviour. Speculative, a similar process is set in motion after RYGB. In RYGB patients, aversive consequences associated with consumption of sugary and fatty foods (e.g. nausea, lightheadedness, flushing, and diarrhoea; (Berthoud et al., 2011) could lead to a relative preference for low-energy food products. Around two months after surgery we see a neural food cue response that is in line with a more habitual rather than a goal-directed pattern. 
Altered appetite-related hormone concentrations were proposed to mediate the changes in neural processing observed after RYGB (Pedersen, 2013; Puzziferri et al., 2016; Svane et al., 2016) and could ultimately contribute to beneficial changes in food preference and intake (Berthoud et al., 2011). In this study we did not find pre- to post-surgery differences in plasma ghrelin concentrations, which might be due the large variation in pre- to post surgery differences in plasma ghrelin concentrations, as also seen in previous studies (Borg et al., 2006; Faulconbridge et al., 2016). Faulconbridge et al (Faulconbridge et al., 2016) found a correlation between changes in ghrelin concentrations and changes in neural responses, which our data do not confirm. Discrepancies in neural activation and ghrelin concentrations could be related to a difference in the timing of the measurements (6 (Faulconbridge et al., 2016) vs 2 months post-surgery in the current study), as the effect of RYGB on ghrelin concentrations might change over time (Saliba et al., 2009).

Endocannabinoids have been suggested to mediate the orexigenic effects of ghrelin (Kola et al., 2008) and are implicated in reward anticipation (Horder et al., 2010; Kroemer et al., 2013; Monteleone et al., 2012; Rigamonti et al., 2015; Tudge et al., 2015). Increased eCB concentrations have been suggested to be a cause of obesity (Engeli et al., 2005) and may be related to long-term weight loss and weight-regain after RYGB. Within the range of eCBs that we measured, we showed increased plasma anandamide concentrations after compared to before RYGB. Concentrations of the other eCBs we measured did not change. Previous studies assessing eCBs at alternate time points after RYGB included only few eCBs and suggest either decreased or unchanged anandamide concentrations, and unchanged concentrations of 2-AG, OEA and PEA (Mallipedhi et al., 2015; Montecucco et al., 2015; Quercioli et al., 2013). Similar to ghrelin, eCB concentrations may change over time and future studies should monitor the progression of eCB concentrations at different time points after RYGB.

It can be seen as limitation that blood samples for plasma eCB and ghrelin concentration determination were drawn after at least three hours of fasting, whereas the fMRI measurements were performed after a lunch meal. Concentrations directly after this meal may have been more closely associated with the neural responses we observed in a satiated state. A particular strength of this study was that participants were measured in a satiated state, to mimic overeating situations more realistically. However, this may have reduced reward related neural activation, and complicates direct comparisons to previous literature (e.g. (Geliebter, 2013; Ochner et al., 2012b). Moreover, most other studies looked into pre- to post-surgery changes in the relative difference between high-energy and low-energy food cues (Ochner et al., 2011; Ochner et al., 2012a; Ochner et al., 2012b), whereas we examined the changes in response to HFHS and LFLS cues relative to baseline, as well as compared to non-food cues, to be able to draw more specific conclusions.

The neural changes we find are in line with the observed increased preferences for lowenergy, and decreased preferences for high-energy foods. The current results suggest that there is no relation between changes in ghrelin and endocannabinoid concentrations, and changes in neural responses to food cues in RYGB patients. Using olfactory food cues in addition to pictures did not reveal new information, but highlights the importance of odours for the anticipation of food. To conclude, RYGB leads to alterations in activation of the frontoparietal network, involved in cognitive control during (food) cue processing. 


\section{Acknowledgments}

The use of the 3T fMRI facility has been made possible by CAT-AgroFood (Shared Research Facilities Wageningen UR). We would like to express our thanks to IFF and AllSens for supplying the odorants. We are grateful to the staff from Rijnstate hospital for facilitating participant recruitment and to Lieke van Genderen, Louise Mulder, Monique Zwinkels and Elbrich Postma who were involved in conducting the measurements for this study. Thanks to Els Siebelink, Jose Bluemer and Annemarie Kruitwagen for their help in composing the standardized meals. HZ and SdB contributed to all parts of the manuscript. GJ, CdG and SB supervised the project, designed the research and were involved in writing the paper. PS contributed to the design of the fMRI paradigm, the analysis of the fMRI data and was involved in writing the paper. IJ and WS facilitated participant recruitment at Rijnstate hospital and were involved in writing the paper. All authors read and approved the final manuscript. The authors declare no conflicts of interest. 
Supplementary Table 6.1. Composition of the standardized meal.

\begin{tabular}{lcccc}
\hline & \multicolumn{2}{c}{ Men } & \multicolumn{2}{c}{ Women } \\
\cline { 2 - 5 } & Pre & Post & Pre & Post \\
\hline Bread Roll ( Wheat bread ( $\pm 22 \mathrm{~g} /$ roll $))$ & $4 \mathrm{pcs}$ & $2 \mathrm{pcs}$ & $3 \mathrm{pcs}$ & $1 \mathrm{pc}$ \\
Margarine (Low-fat) & $30 \mathrm{~g}$ & $15 \mathrm{~g}$ & $15 \mathrm{~g}$ & $15 \mathrm{~g}$ \\
Cheese (Full-fat semi-cured) & $40 \mathrm{~g}$ & $20 \mathrm{~g}$ & $40 \mathrm{~g}$ & $20 \mathrm{~g}$ \\
Ham & $40 \mathrm{~g}$ & $20 \mathrm{~g}$ & $20 \mathrm{~g}$ & - \\
Orange Juice & $150 \mathrm{~g}$ & $75 \mathrm{~g}$ & $100 \mathrm{~g}$ & $50 \mathrm{~g}$ \\
kCal total meal & 570 & 174 & 421 & 107 \\
\hline
\end{tabular}

Supplementary Table 6.2. Stimulus ratings for liking, wanting and intensity pre- and post-RYGB surgery

\begin{tabular}{lllccc}
\hline & & Category & $\begin{array}{c}\text { Before } \\
\text { surgery }\end{array}$ & $\begin{array}{c}\text { After } \\
\text { surgery }\end{array}$ & Significance \\
\hline PICTURE & Liking & $H F H S$ & $46 \pm 5$ & $30 \pm 5$ & $p<0.001$ \\
& & LFLS & $39 \pm 6$ & $43 \pm 6$ & $p=0.246$ \\
& & $N F$ & $19 \pm 4$ & $14 \pm 4$ & $p=0.069$ \\
\cline { 2 - 6 } & Wanting & $H F H S$ & $42 \pm 5$ & $26 \pm 5$ & $p<0.001$ \\
& & LFLS & $35 \pm 6$ & $37 \pm 6$ & $p=0.498$ \\
& & $N F$ & $10 \pm 3$ & $8 \pm 3$ & $p=0.244$ \\
\hline ODOUR & Liking & $H F H S$ & $49 \pm 7$ & $29 \pm 7$ & $p<0.001$ \\
& & LFLS & $39 \pm 6$ & $37 \pm 6$ & $p=0.798$ \\
& & $N F$ & $35 \pm 7$ & $23 \pm 7$ & $p=0.008$ \\
\cline { 2 - 6 } & Wanting & $H F H S$ & $38 \pm 6$ & $24 \pm 6$ & $p=0.018$ \\
& & $L F L S$ & $33 \pm 6$ & $35 \pm 6$ & $p=0.804$ \\
& & $N F$ & $14 \pm 4$ & $9 \pm 3$ & $p=0.191$ \\
\cline { 2 - 5 } & Intensity & $H F H S$ & $76 \pm 5$ & $76 \pm 4$ & $p=0.984$ \\
& & $L F L S$ & $60 \pm 5$ & $66 \pm 5$ & $p=0.147$ \\
& & $N F$ & $68 \pm 5$ & $73 \pm 5$ & $p=0.114$ \\
\hline
\end{tabular}

Ratings performed on a 100 point VAS. Values are means \pm SD. HFHS: high-fat/high-sugar; LFLS: low-fat/lowsugar; NF: non-food; RYGB: Roux-en-Y gastric bypass. 


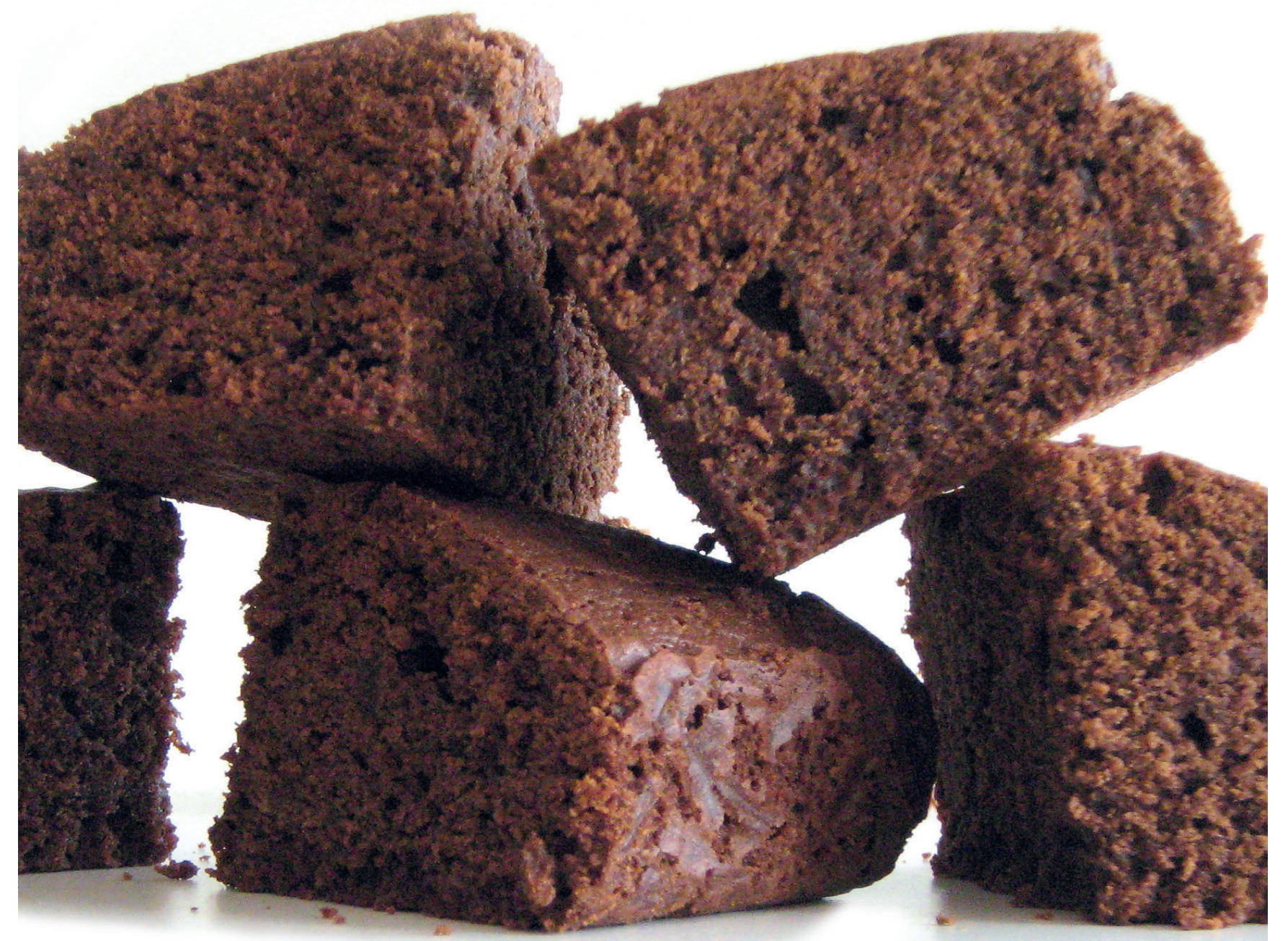




\section{CHAPTER 7}

Altered neURAL INHiBITION RESPONSES tO FOOD CUES AFTer RouX-EN-Y GASTRIC BYPASS

Harriët F.A. Zoon

Suzanne E.M de Bruijn

Gerry Jager

Paul A.M. Smeets

Cees de Graaf

Ignace M.C. Janssen

Wendy Schijns

Laura N. Deden

Sanne Boesveldt

Submitted. 


\begin{abstract}
BACKGROUND: Roux-en-Y gastric bypass (RYGB) surgery is a highly effective weight-loss intervention that often reduces preference and intake of high-energy foods. Research into the neural mechanisms behind this shift has mainly focused on reward processing of food cues. However, the ability to successfully control food intake and thereby weight-loss also depends on inhibitory control capacity. We investigated whether RYGB leads to alterations in neural inhibitory control in response to food cues.

METHODS: A food-specific go/no-go task with pictures of high-energy (desserts) and low-energy foods (vegetables), was used to assess neural inhibition responses before and after RYGB with functional magnetic resonance imaging. Data from 18 morbidly obese patients (15 females; age $41 \pm 11$ years; BMI $42 \pm 4 \mathrm{~kg} / \mathrm{m}^{2}$ before; BMI $36 \pm 4 \mathrm{~kg} / \mathrm{m}^{2}$ after) were analysed. Pre- and post-RYGB BOLD fMRI responses were compared for response inhibition towards high-energy and for response inhibition towards low-energy foods. Participants were tested in a satiated state.

RESULTS: Response inhibition for high-energy food was associated with increased activation of the right anterolateral prefrontal cortex (PFC), right medial $\mathrm{PFC}$, dorsolateral $\mathrm{PFC}$, right middle cingulate cortex and the right inferior frontal operculum, after compared to before surgery (involved in inhibitory control). Response inhibition for low-energy food elicited diminished post- compared to pre-surgery responses in the left superior temporal pole, right parahippocampal gyrus and right hypothalamus (involved in metabolic control). The dorsolateral PFC, middle cingulate cortex, inferior frontal operculum, parahippocampal gyrus and superior temporal pole have also been associated with reward processing.

CONCLUSION: Neural changes indicate improved response inhibition towards high-energy food cues, altered influence of satiety during response inhibition toward low-energy food cues and a more positive attitude to both high-energy and low-energy food after RYGB. Alterations in neural circuits involved in inhibitory control, satiety signalling and reward processing may contribute to effective weight-loss after RYGB.
\end{abstract}




\section{Introduction}

Roux-en-Y gastric bypass (RYGB) patients show decreased preferences and consumption of high-energy foods, which are associated with long-term weight reduction (Kenler et al., 1990; Laurenius et al., 2013; Ochner et al., 2011; Sjöström, 2013; Thirlby et al., 2006). The underlying mechanism of this decreased preference for high-energy foods is unclear. Most studies to date focused on altered reward processing, but changes in inhibitory control may also play an important role. It has been suggested that people with low inhibitory control are more prone to overeating and hence to developing overweight or obesity (Guerrieri et al., 2008; Nederkoorn et al., 2009; Weygandt et al., 2015). Suppression of automatic tendencies to choose highly rewarding energydense foods over low energy-dense foods could help to decrease caloric intake, which contributes to successful weight-loss.

How well we are able to control our impulses in part determines how much and what we consume. Decreased inhibitory control is assumed to increase the odds of eating in the absence of hunger, especially in a tempting and food-rich environment (Boutelle and Bouton, 2015; Meule et al., 2014; Volkow et al., 2011), and could eventually lead to weight gain. Overeating and obesity have been associated with higher impulsivity, both in self-reported and behavioural measures (Bongers et al., 2015; Dykes et al., 2004; Nederkoorn et al., 2006; Ryden et al., 2003; Stoeckel et al., 2007; Vainik et al., 2013). Furthermore, individuals that were unsuccessful in regulating their weight show decreased inhibitory control (Houben et al., 2012) while behavioural responses of successful weight-loss maintainers to high-energy foods indicate better inhibition (Phelan et al., 2011). The extent of inhibitory control seems to influence the ability to maintain weight-loss after intervention. RYGB surgery is widely viewed as the most effective method for long-term weight loss in morbidly obese individuals (Rubino et al., 2004).

Previous studies into neural responsivity after RYGB surgery have mainly focused on (alterations in) reward processing during presentation of high-energy food items (Ochner et al., 2011; Ochner et al., 2012a). In order to better understand successful weight-loss regulation upon RYGB, it is important to consider changes in inhibitory control processes as well (Price et al., 2015).

Previous studies showed that people who were attempting to lose weight displayed increased activation of the inferior frontal gyrus and anterior insula/frontal operculum in response to pictures of high-energy foods (Smeets et al., 2013). These areas are involved in inhibitory control. Also, successful weight loss maintainers show greater activation to food cues in prefrontal regions (superior-, middle frontal gyrus) associated with inhibitory control (McCaffery et al., 2009). Batterink et al. (Batterink et al., 2010) have introduced a food-specific go/no-go task to assess neural measures of response inhibition to food items. In their study, a higher BMI was related to less activation during no-go trials in frontal inhibitory regions, including superior- and middle frontal gyrus, ventromedial- and medial prefrontal cortex, and orbitofrontal cortex. A higher BMI was also associated with more activation during no-go trials in the temporal operculum. Increased understanding of the (neuro)biological mechanisms involved in inhibitory control is necessary to improve the outcome of weight-loss interventions.

With this study we aimed to determine whether RYGB surgical intervention in morbidly obese patients results in altered neural activation underlying response inhibition, using a food 
specific go/no-go task. Participants were tested in a satiated state to better understand alterations in mechanisms of overeating. We hypothesized that participants would be better able to suppress responses to high-energy (dessert) items after RYGB surgery, as reflected in changes in neural responses related to inhibitory control, while behavioural and neural responses to low-energy (vegetable) items would remain similar.

\section{Methods}

\section{Overall design}

This study had a 2x2x2 within-subject design, including the factors time point (pre- and post-RYGB), stimulus (dessert/vegetable), and task-instruction (go/no-go).

Participants

Twenty morbidly obese individuals participated in the food-specific go/no-go task, pre- and post- RYGB surgery. All participants were enlisted to undergo RYGB surgery at Rijnstate hospital, Arnhem, the Netherlands. Requirements for the surgery were: Body Mass Index (BMI) of $>40 \mathrm{~kg} / \mathrm{m}^{2}$ or $>35 \mathrm{~kg} / \mathrm{m}^{2}$ with co-morbidity that was expected to improve after surgically-induced weight loss, long-lasting obesity ( $>5$ years), proven failed attempts to lose weight in a conventional way, intention to adhere to a postoperative follow-up programme. Individuals were not considered for surgery when they were pregnant or lactating, had psychiatric disorders, alcohol or drug dependency, life threatening conditions or when they were dependent on the care of others. Patients were screened at Rijnstate hospital. All participants were right-handed, non-smoking, and did not have conditions that conflicted with MR safety or would cause artefacts in the MR images (e.g. claustrophobic, irremovable ferromagnetic objects in or on their body, pacemaker). Participants received financial compensation for their contribution. All participants provided written informed consent before entering the study. The protocol was approved by the Medical Ethical Committee of Wageningen University (NL45837.081.13) and was executed in accordance with the ethical principles of the Declaration of Helsinki of 1975, as revised in 2013. The study was registered on clinicaltrials.gov as NCT02068001.

\section{Experimental procedures}

Participants visited the test facilities at three occasions. First, they were familiarized with the MRI test environment and the experimental task in a dummy MRI scanner at Wageningen University (training session). After the trainings session, actual measurements were performed in two identical test sessions. The first test session took place on average $3.3(\mathrm{SD}=1.8)$ weeks before, and the second test session took place on average $9.3(\mathrm{SD}=1.2)$ weeks after RYGB surgery. Participants were instructed to refrain from eating and drinking anything but water and weak tea in the three hours before the test sessions. Upon arrival at hospital Gelderse Vallei (Ede, the Netherlands), blood samples were taken for analysis of plasma levels of endocannabinoids and ghrelin (data reported elsewhere). Participants were tested in comfortably full state, to mimic a context of eating in the absence of hunger. We provided a standardized meal that was adapted to pre- or post-surgery conditions in order to match the hunger states of the participants before and after surgery. Participants first drank orange juice, and after a small break they consumed a 
standardized meal consisting of bread roll(s), cheese, ham and butter (see Supplementary Table 1). Following meal consumption, participants waited for 15 minutes. In order to assess changes in general inhibition participants filled in the 24-item BIS/BAS questionnaire (Carver and White, 1994). Measurements of brain reward responses to visual and olfactory food and non-food cues were collected (Chapter 6). At the end of this reward paradigm participants rated their appetite (hunger, fullness, prospective consumption, desire to eat, and thirst) on a 100 point visual analogue scale (VAS). Then a structural MR image was collected. Finally, participants took part in two functional runs during which a food-specific go/no-go task was performed.

\section{fMRI - go/no-go task}

The food-specific go/no-go paradigm was adapted from Batterink et al. (Batterink et al., 2010). Participants were instructed to press a button as quickly and accurately as possible in response to go trials ( $75 \%$ occurrence) and to refrain from responding to no-go trials ( $25 \%$ occurrence). Two separate functional runs were performed, each consisting of 48 trials. One run contained govegetable items and no-go dessert items, the other run contained go-dessert items and no-go vegetable items (see Figure 7.1). The order of the runs was counterbalanced between participants. During each trial a picture was presented for $500 \mathrm{~ms}$, depicting either a low-energy vegetable (i.e. corn, peas, Brussels sprouts, radishes, carrot, broccoli, cauliflower, haricots, zucchini) or a highenergy dessert (i.e. ice cream, cake, frozen yogurt, pudding, chocolate mousse, chocolates, cookies). Participants had $2000 \mathrm{~ms}$ to respond from stimulus onset. Trials were presented in pseudorandomized order. Between trials a fixation cross was presented for a duration of 7-19 seconds. Nogo trials would appear after 1, 2, or 3 go-trials. Reaction times were measured from the beginning of trial onset and collected with a fibre-optic response box system. Stimuli were presented visually using the Presentation software package (Version 9, Neurobehavioral Systems, Davis, CA) and were displayed using a video projector that illuminated a rear projection screen located at the end of the magnet bore. Subjects viewed the stimuli through an adjustable mirror attached to the head coil. 


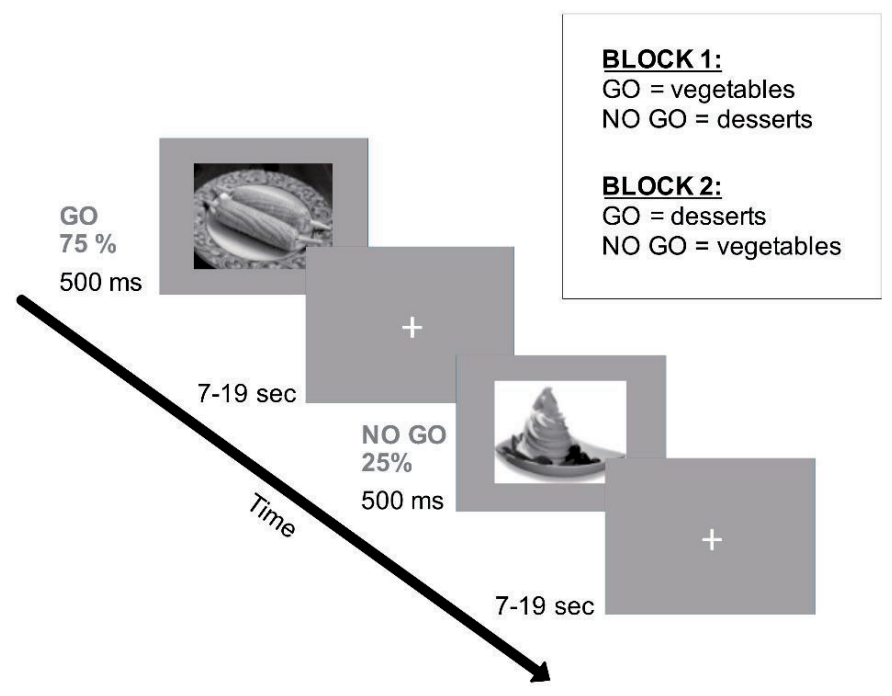

Figure 7.1. The food-specific Go/No-Go paradigm. In block A. participants were instructed to press a button in response to vegetable items (36 items) and withhold their response to dessert items (12 items). In block B. the instruction was reversed, participants had to press the button in response to dessert items (36 items) and withhold their response to vegetable items (12 items). The block order was counterbalanced between participants.

\section{(f)MRI measurements}

Each participant was scanned at approximately the same time of day, between 14:00-17:00 at hospital Gelderse Vallei (Ede, the Netherlands). Images were acquired with a 3-Tesla Siemens Magnetom Verio MRI scanner in combination with a 32-channel head coil. A high-resolution T1weighted anatomical MRI scan was acquired (MPRAGE: repetition time $=1900 \mathrm{~ms}$, echo time $=2.26$ $\mathrm{ms}, 9^{\circ}$ flip angle, field of view $=256 \times 256 \mathrm{~mm}, 192$ sagittal slices, voxel size $=0.5 \times 0.5 \times 1 \mathrm{~mm}$ ). Subsequently, $176 \mathrm{~T}^{\circ}$-weighted gradient echo images with BOLD contrast (repetition time $=2240$ $\mathrm{ms}$, echo time $=25 \mathrm{~ms}, 90^{\circ}$ flip angle, field of view $=192 \times 192 \mathrm{~mm}, 45$ axial slices, ascending order, voxel size $3 \times 3 \times 3 \mathrm{~mm}$ ) were acquired for each of the two functional runs during which participants performed a food Go/No-Go task. The imaging volume was tilted at an oblique angle of $30^{\circ}$ to the anterior-posterior commissure line to reduce signal dropout in the orbitofrontal and ventral temporal lobes (Deichmann et al., 2003). Head movements were restricted by placing foam cushions next to the participants' head. In addition, adhesive tape was placed across the participants' forehead to provide feedback on head movements. Earplugs were provided for noise reduction.

\section{Data analyses}

Participant characteristics. Participant characteristics were analysed using SPSS in IBM SPSS Statistics for Windows, Version 22.0 (IBM Corp., Armonk, NY, USA). Paired-samples T-tests were used to test differences in weight, BMI, hunger ratings and BIS/BAS-scores pre- and postsurgery. 
Behavioural data go/no-go. Behavioural data of the go/no-go task were also analysed using SPSS. Mean commission error rates of the go/no-go task were calculated by dividing the total number of incorrect responses to no-go trials by the total number of no-go trials. Mean omission error rates were calculated by dividing the total number of non-responses to go-items by the total number of go-trials. Mean reaction times (ms) of responses to each type of trial (go-dessert, govegetable, no-go dessert, no-go vegetable) were calculated for each participant. Response times below $200 \mathrm{~ms}$ and over $2000 \mathrm{~ms}$ were excluded. The low number of commission errors rendered the reaction time data for the no-go items unsuitable for statistical testing. Pre- to post-surgery differences in response time (ms) to go items were analysed by following a linear Mixed Effects Models procedure including stimulus type (go-dessert; go-vegetable) as fixed effects factor. Time point (pre- and post-gastric bypass surgery), stimulus (dessert/vegetable), and task-instruction (go/no-go) were included as repeated variables. A p-value of $<0.05$ was considered statistically significant.

fMRI data go/no-go. Whole brain functional images were pre-processed and analysed using the SPM12 software package (Wellcome Department of Imaging Neuroscience, London, United Kingdom) run within MATLAB 7.12.0 (R2011a, The Mathworks Inc). Functional images were slice timed, realigned and coregistered. The DARTEL framework was used to create a study-specific template and participant-specific deformation fields (Ashburner, 2007). The images were then spatially normalized to the Montreal Neurological Institute (MNI) standard brain using the studyspecific DARTEL template and the participant-specific deformation fields. Smoothing was applied to the normalized images using an isotropic Gaussian kernel with a 6-mm full width at half maximum. Artefact Repair was applied using the ArtRepair toolbox in SPM12 (see: http://cibsr.stanford.edu/tools/human-brain-project/artrepair-software.html). Of the twenty datasets that were acquired, two datasets were excluded that contained movements more than 4 $\mathrm{mm}$.

Subject level analyses. Each test session (pre-/post-surgery) was modelled separately. Four conditions were included per model: visual exposure to go dessert trials, no-go vegetable trials, go vegetable trials and no-go dessert trials. Motion-correction parameters were included in the model. For each subject four contrast images were calculated: nogo_dessertpre vs rest, nogo_dessertpost vs rest, nogo_vegetablepre vs rest and nogo_vegetablepost vs rest. Subsequently we subtracted the postsurgery contrast images from the pre-surgery contrast images using the SPM12 image calculation routine.

Group level analyses. Two one-sample T-tests were performed to test our hypotheses. In each test we looked at contrast images containing the difference between activations pre- and postsurgery (nogo_dessertpre-nogo_dessertpost; nogo_vegetablepre-nogo_vegetable post$_{\text {) }}$. We report whole brain results, with a significance level of $p=0.001$ (unc.) and a cluster extent threshold of $\mathrm{k}=8$ contiguous voxels. The MarsBar toolbox (http://marsbar.sourceforge.net/) run in Matlab 7.12.0 (R2011a; The Mathworks Inc., Natick, MA) was used to extract mean beta values from all significant clusters. These values were subsequently correlated with pre- to post- surgery changes in BMI, changes in body weight, and changes in feelings of hunger, fullness, prospective consumption and desire to eat. Correlation analyses were performed in SPSS using Pearson's correlation coefficient. 


\section{Results}

\section{RYGB effects - weight loss}

The mean weight of our study population decreased from $121 \pm 15$ pre-RYGB to $105 \pm 16 \mathrm{~kg}$ post-RYGB (mean $\pm \mathrm{SD}$ ), a mean weight loss of $17 \pm 3 \mathrm{~kg}(p<.001)$. This weight change led to a decrease in BMI from $42 \pm 4$ to $36 \pm 4 \mathrm{~kg} / \mathrm{m}^{2}(p<0.001)$, with a mean decrease of $6 \pm 1 \mathrm{~kg} / \mathrm{m}^{2}$.

\section{Hunger ratings}

During the post-surgery test session, participants indicated less hunger before the go/no-go task ( $\sim 50$ min after meal intake; $p=0.056$ ), rated a higher fullness, a decreased prospective consumption and less desire to eat (all $p<0.01$ ). Ratings for thirst were comparable between the two test sessions $(p=0.349$; see Table 7.1$)$.

Table 7.1. Weight, BMI, Hunger ratings provided right before the go/no-go task and BIS/BAS scores before and after RYGB surgery.

\begin{tabular}{lccc}
\hline & $\begin{array}{c}\text { Before } \\
\text { surgery }\end{array}$ & After surgery & Significance \\
\hline Weight $(\mathrm{kg})$ & $121 \pm 15$ & $105 \pm 16$ & $p<0.001$ \\
BMI $\left(\mathrm{kg} / \mathrm{m}^{2}\right)$ & $42 \pm 4$ & $36 \pm 4$ & $p<0.001$ \\
\hline Hunger & $27 \pm 31$ & $14 \pm 24$ & $p=0.056$ \\
Fullness & $43 \pm 29$ & $72 \pm 18$ & $p=0.001$ \\
Prospective consumption & $37 \pm 27$ & $17 \pm 21$ & $p=0.002$ \\
Desire to eat & $44 \pm 35$ & $24 \pm 26$ & $p=0.009$ \\
Thirst & $76 \pm 26$ & $70 \pm 28$ & $p=0.349$ \\
\hline BAS Drive (max 16) & $11.6 \pm 2.1$ & $12.1 \pm 2.2$ & $p=0.132$ \\
BAS Fun Seeking $(\max 16)$ & $10.8 \pm 2.1$ & $11.3 \pm 2.3$ & $p=0.166$ \\
BAS Reward Responsiveness $(\max 20)$ & $17.8 \pm 1.6$ & $18.4 \pm 1.6$ & $p=0.045$ \\
BIS (max 28) & $20.3 \pm 4.4$ & $19.8 \pm 3.6$ & $p=0.366$ \\
\hline
\end{tabular}

Hunger ratings performed on a 100 point VAS. Values are means \pm SD. BMI = Body mass index.

\section{Behavioural data}

No-go items. Commission errors (incorrect responses during the no-go items) for no-go dessert items occurred at a mean rate of $8.5 \%(\mathrm{SD}=8.6)$ pre-surgery, and $8.8 \%(\mathrm{SD}=10.9)$ postsurgery. Commission errors for no-go vegetable items occurred around $16.8 \%(\mathrm{SD}=15.8)$ presurgery, and $14.8 \%(\mathrm{SD}=14.7)$ post-surgery.

The go/no-go task included 12 no-go items per run. Commission errors to no-go dessert items occurred at $443 \pm 87 \mathrm{~ms}($ mean $\pm \mathrm{SD})$ pre-surgery and at $501 \pm 132$ post-surgery. Commission errors in response to no-go vegetable items had a mean reaction time of $526 \pm 125 \mathrm{~ms}$. After surgery, responses to no-go vegetable items occurred at $504 \pm 143$.

Go items For the go dessert items, the omission error rate (non-responses during the gotrials) changed from $0.9 \%(\mathrm{SD}=1.7)$ before surgery to $1.7 \%(\mathrm{SD}=2.5)$ after surgery. The mean rate of omission errors for go vegetable items was $4.0 \%(\mathrm{SD}=2.7)$ before surgery and $3.4 \%(\mathrm{SD}=2.0)$ after surgery. 
There were no significant differences between reaction times to go dessert items before $(543 \pm 90 \mathrm{~ms} ;$ mean $\pm \mathrm{SD})$ versus after RYGB $(567 \pm 122 \mathrm{~ms} ; p=0.395)$, nor between reaction times to go vegetable items before $(544 \pm 138 \mathrm{~ms})$ and after RYGB $(538 \pm 135 \mathrm{~ms} ; p=0.395)$.

\section{Functional imaging data}

No-go desserts. Comparisons between pre- and post-surgery fMRI BOLD responses for the no-go dessert trials revealed increased post-surgical activation of the right middle frontal gyrus (lateral part), the medial part of the right superior frontal gyrus, the right inferior frontal gyrus (pars triangularis), the right middle cingulum and the inferior frontal operculum (see Table 7.2 and Figure 7.2). There were no regions in which no-go activation was significantly decreased post- compared to pre-surgery.

There were no significant correlations between pre- to post-surgery changes in neural responses during no-go dessert trials and changes in BMI or body weight, changes in feelings of hunger, fullness, prospective consumption and desire to eat (all $p>0.05$ ).

Table 7.2. Regions in which brain activation during go or no-go food items was significantly different pre- and post RYGB surgery

\begin{tabular}{|c|c|c|c|c|c|c|c|}
\hline & & & \multirow[t]{2}{*}{$\begin{array}{c}\text { cluster } \\
\text { size }\end{array}$} & \multirow[t]{2}{*}{ Z-score } & \multicolumn{3}{|c|}{ Peak coordinates } \\
\hline & & & & & $\mathbf{x}$ & $\mathrm{y}$ & $\mathbf{z}$ \\
\hline \multicolumn{8}{|l|}{ DESSERT } \\
\hline \multirow[t]{5}{*}{ no-gopre $<$ no-gopost } & $\mathrm{R}$ & Middle Frontal Gyrus / Anterolateral PFC & 30 & 4.42 & 45 & 54 & 6 \\
\hline & $\mathrm{R}$ & Medial Superior Frontal Gyrus / Medial PFC & 23 & 4.02 & 12 & 60 & 27 \\
\hline & $\mathrm{R}$ & Inferior Frontal Gyrus (Tri) / Dorsolateral & & & & & \\
\hline & & $\mathrm{PFC}$ & 15 & 3.87 & 57 & 27 & 18 \\
\hline & $\mathrm{R}$ & Middle Cingulum (posterior part) & 10 & 3.59 & 3 & -27 & 33 \\
\hline \multicolumn{8}{|l|}{ VEGETABLE } \\
\hline \multirow[t]{3}{*}{ no-gopre > no-gopost } & $\mathrm{R}$ & Hypothalamus & 10 & 3.65 & 3 & 3 & -12 \\
\hline & $\mathrm{L}$ & Superior Temporal Pole & 11 & 3.63 & -36 & 12 & -27 \\
\hline & $\mathrm{R}$ & Parahippocampal gyrus & 9 & 3.34 & 18 & -15 & -21 \\
\hline
\end{tabular}

No-go vegetables. Pre-surgical neural activation to no-go vegetable items was significantly higher in the right hypothalamus, left superior temporal pole and right parahippocampal gyrus, relative to post-surgery (see Table 7.2 and Figure 7.3). There were no regions in which activation was significantly increased post- compared to pre-surgery.

Pre- to post-surgery changes in activation of the right parahippocampal gyrus during no-go vegetable items were correlated with changes in ratings of fullness provided right before the go/nogo task $(\mathrm{r}=-0.625, p=0.007)$. No significant correlations were found between pre- to post-surgery changes in neural responses during no-go vegetable trials and BMI or body weight, and changes in feelings of hunger, prospective consumption and desire to eat (all $p>0.05$ ). 


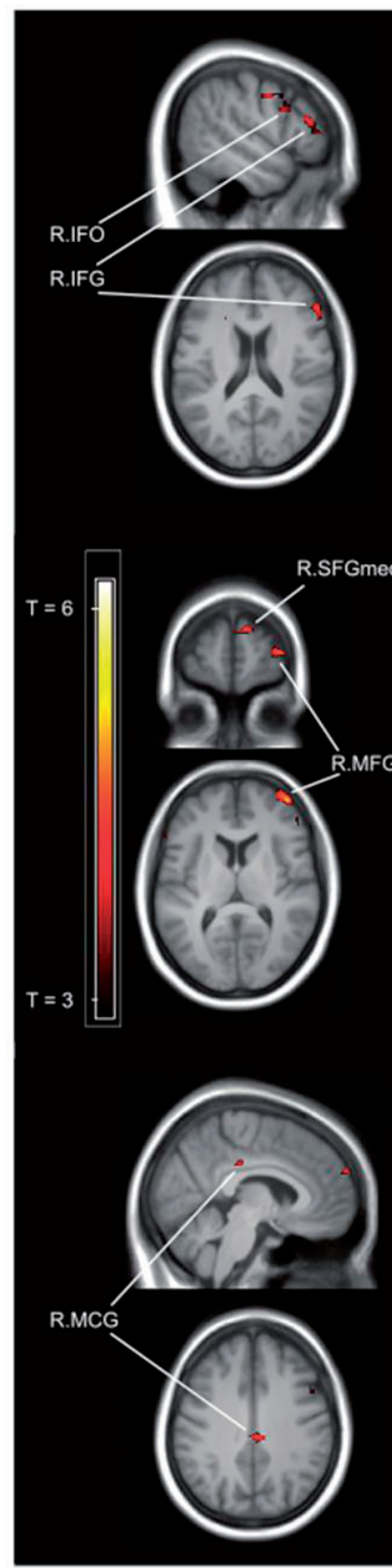
R. Inferior Frontal Operculum (MNI 519 24)
R. Inferior Frontal Gyrus (MNI 5727 18)
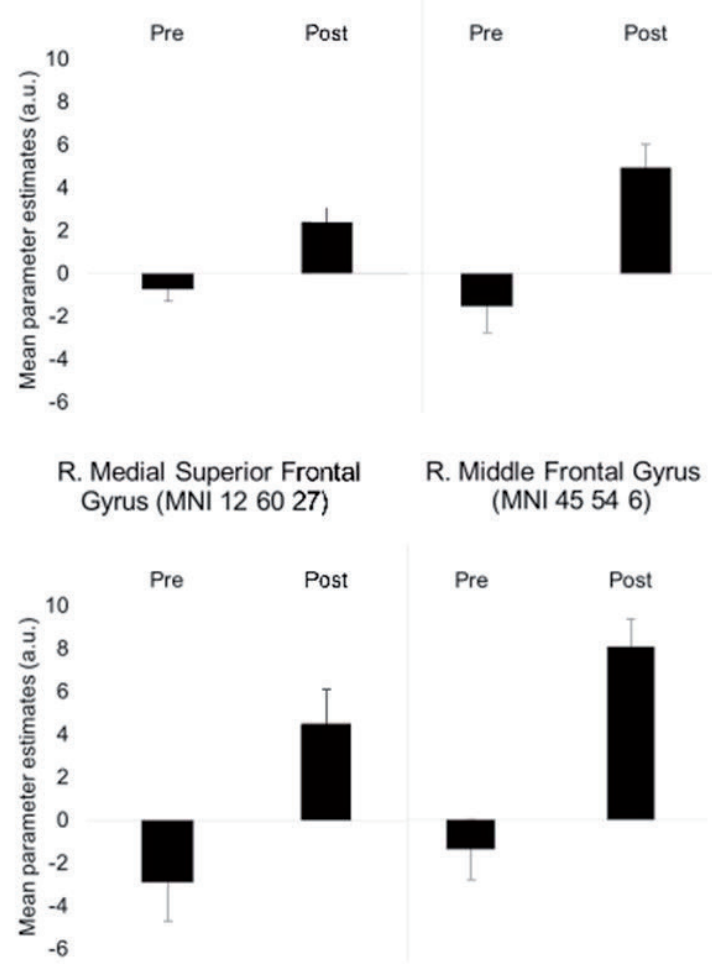

R. Middle Cingulate Gyrus (MNI 3 -27 33)

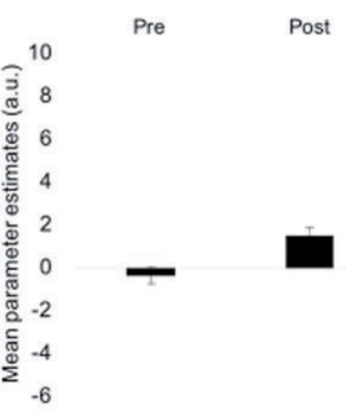

Figure 7.2. Regions in which brain activation during response inhibition to dessert items was significantly different pre- and post RYGB surgery. Brain images were thresholded at $p=0.005$ for visualisation. Upper. The right inferior frontal gyrus (Tri; MNI: 5727 18) was more activated after compared to before surgery and the right inferior frontal operculum (MNI: 51924 ) showed more activation after compared to before surgery. Middle: The right middle frontal gyrus (MNI: 45546 ) was more activated after than before surgery and the right medial superior frontal gyrus (MNI: 12 60 27) showed deactivation before surgery and activation after surgery. Lower. The right middle cingulate cortex (MNI: 3 -27 33) was more activated after surgery. 


\section{R. Hypothalamus}

(MNI 33 -12)

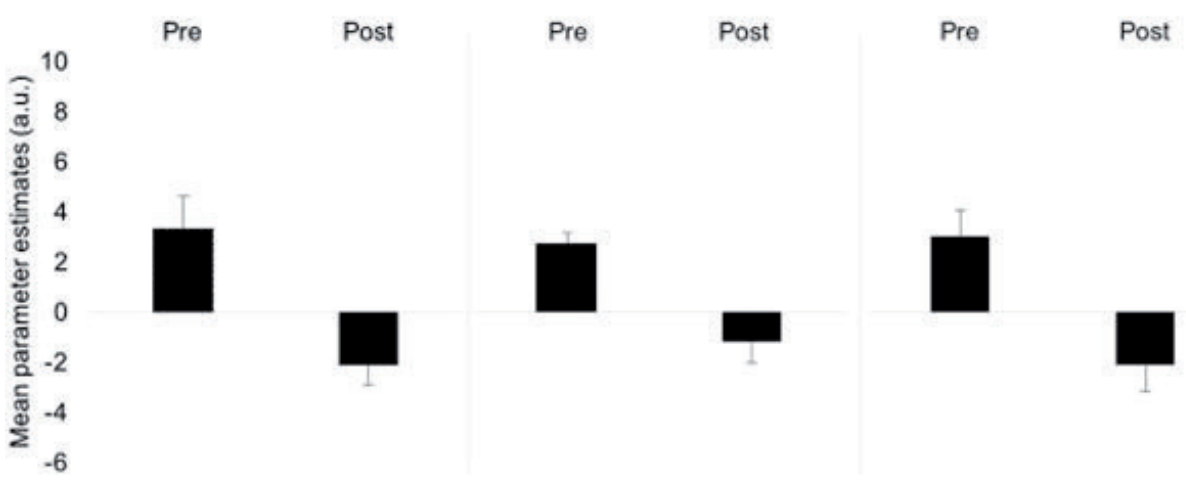

L. Superior Temporal Pole
(MNI -36 12 -27)
R. Parahippocampal Gyrus

(MNI $18-15-21$ )

\author{
(1)
}

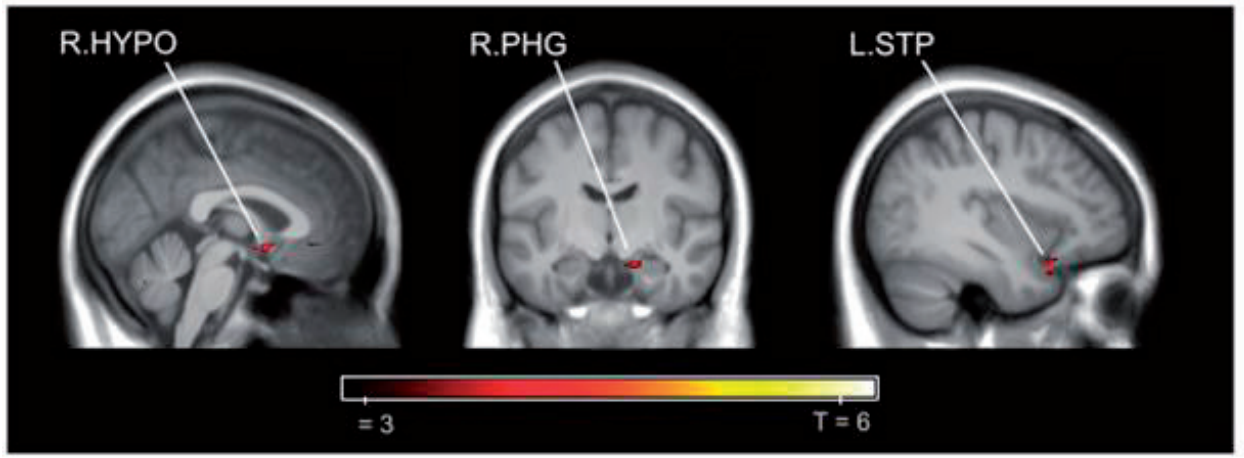

Figure 7.3. Regions in which brain activation during response inhibition to vegetable items was significantly reduced post- compared to pre-RYGB surgery. Brain images were thresholded at $p=0.005$ for visualisation. Left: The right hypothalamus (MNI: 33 -12) was activated before surgery and deactivated after surgery. Middle: The right parahippocampal gyrus (MNI: 18 -15-21) displayed activation before and deactivation after surgery. Right. The left superior temporal pole (MNI: -36 12 -27) showed activation before surgery and deactivation after surgery.

\title{
Discussion
}

To our knowledge, this is the first study to investigate changes in neural inhibition to food cues after RYGB. We found pre- to post-surgery increases in neural response to no-go high-energy dense food items in regions involved in inhibitory control (middle, medial superior- and inferior frontal gyrus). Further, neural activation in response to no-go low-energy dense food items was less pronounced in regions related to satiation (hypothalamus, parahippocampal gyrus, superior temporal pole) after surgery. Alterations in reward related activation were found for both no-go dessert and no-go vegetable trials (inferior frontal gyrus, middle cingulate gyrus, inferior frontal operculum, parahippocampal gyrus, superior temporal pole).

As expected, neural activation to no-go vegetable items did not change after surgery in regions involved in inhibitory control. During response inhibition towards desserts, however, we observed increased involvement of prefrontal regions (middle-, medial superior- and inferior frontal 
gyrus) after surgery. Previous research has linked increased activation in these regions to greater exertion and success of inhibitory control (Chikazoe et al., 2009; Hutcherson et al., 2012; Kober et al., 2010; Li et al., 2006; Scharmuller et al., 2012; Sebastian et al., 2012; van der Meer et al., 2011). This suggests that activation in these frontal regions can serve as an indicator for response inhibition capacity. Interestingly, Lapenta et al. found that it is possible to induce changes in response inhibition processes by transcranial direct current stimulation (tDCS) of the dorsolateral prefrontal cortex (dlPFC; Lapenta et al., 2014). They showed that this type of neural stimulation leads to significant changes in neural markers of inhibitory control, and also to reduced craving and food intake. In our study, increased prefrontal cortex activation post-surgery could indicate an increase in neural inhibitory control in response to appetizing food items. In contrast to Batterink et al. (Batterink et al., 2010) who found correlations between current BMI and prefrontal activation during inhibitory control, we did not find significant correlations between changes in prefrontal activation and changes in body weight or BMI. This is likely related to greater variation (from lean to obese) in current BMI in their study (Batterink et al., 2010), versus limited variation in withinsubject changes in BMI in the current study. The observed changes in neural processing after RYGB support an improved response inhibition towards high-energy food.

Post-surgical reductions in parahippocampal gyrus, superior temporal pole, and also hypothalamus activation during low-energy no-go items, but not during high-energy no-go items, could relate to metabolic signals of satiety. In the current study, participants were equally satiated directly after meal intake in both test sessions (see Supplementary Table 7.2), but felt less hungry and more full post- compared to pre-surgery before starting the go/no-go task. This could be related to accelerated digestion and absorption of nutrients after RYGB (Bojsen-Moller et al., 2015). Moreover, a significant correlation was found between pre- to post-surgery changes in parahippocampal gyrus activation and changes in ratings of fullness. Previous studies found increased brain activation to high-energy food cues (visual, taste) in the parahippocampal gyrus and hypothalamus in a hungry compared to a satiated state (Haase et al., 2009; LaBar et al., 2001; Leidy et al., 2011; van der Laan et al., 2011), and related this to an increased salience of energy-rich products during hunger (Mohanty et al., 2008; van der Laan et al., 2014). In light of this, the decrease in hypothalamic, parahippocampal and superior temporal pole activation during response inhibition after surgery suggests that the increase in feelings of fullness is related to a decrease in salience of low-energy products, but not high-energy products.

Besides increased activation in prefrontal regions of inhibitory control, we found increased activation in the inferior frontal gyrus, inferior frontal operculum and middle cingulate cortex during response inhibition towards high-energy food. Although, activation in these regions has been linked to selective attention and more effective response (Booth et al., 2003; Cojan et al., 2009; Hirose et al., 2009; Li et al., 2006), these regions are also implicated in processing of reward value and taste evaluation in response to cue exposure during anticipation, consumption (Nummenmaa et al., 2012; Small et al., 2001; Stice et al., 2008b), and self-regulation (Hare et al., 2011; Vollm et al., 2006; Zotev et al., 2011). Our results thus suggest greater engagement of these reward-related areas during response inhibition for high-energy products after surgery. Decreased post-surgery activation of the parahippocampal gyrus and superior temporal pole during response inhibition for low-energy food products could also be associated with changes in reward processing. Increased 
activation in the parahippocampal gyrus during exposure to taste and smell of food was associated with decreasing reward value in healthy (Small et al., 2001) and obese subjects (Bragulat et al., 2010). The observed reduction in parahippocampal gyrus deactivation during response inhibition to lowenergy food cues thus could imply a more positive attitude towards these cues. However, we have no ratings of liking or wanting ratings for the food stimuli, so we can only speculate about a link between the decrease in inhibitory activation and higher preference for low-energy products. Nevertheless, the relative increase in preference for low-energy food found in RYGB patients (Kenler et al., 1990; Ochner et al., 2012b; Thirlby et al., 2006), does support a more positive attitude towards vegetables after surgery.

As mentioned above, the regions in which we find increased activation in response to nogo dessert items after surgery have been linked to increased exertion of neural inhibitory control and also to more successful behavioural inhibition (Chikazoe et al., 2009; Hutcherson et al., 2012; Kober et al., 2010; Li et al., 2006; Scharmuller et al., 2012; Sebastian et al., 2012; van der Meer et al., 2011). It would be interesting to link these neural data to actual behavioural changes. However, the limited amount of no-go trials $(\mathrm{n}=12)$ in the task we used, unfortunately rendered the behavioural data for this condition unsuitable for reliable statistical inferences about correlation to neural outcomes. Further, reaction times to go-dessert and go-vegetable items were not significantly different between the pre- and post-surgery test session. Thus, with the current data we cannot conclude whether the changes we find solely reflect increased exertion of neural inhibitory control or whether they have implications for actual behaviour. Future research including more extensive behavioural measures is needed to clarify the link between changes in neural and behavioural response inhibition in RYGB patients. However, the food specific go/no-go task does approach reallife decision-making processes better than the passive reward tasks that have been used in previous research. Because of limited statistical power due to the small sample size, we have used a relatively lenient threshold for the fMRI analyses. We are aware that this increases the risk of false positive results. Nonetheless, this research provides unique additional insight in the mechanisms underlying the effectivity of RYGB surgery. The within-subject design provides a solid method for testing RYGB related changes. Moreover, unlike most previous research, measurements in this study have been obtained in a satiated state, to better mimic a context of overeating that has a greater ecological relevance in obesity. Despite high effectiveness of RYGB on weight loss and promising results demonstrated in a 20 year follow-up study (Sjöström, 2013), weight regain after more than one year post-surgery is a recurring problem in a subset of patients (Himes et al., 2015). Perhaps additional (cognitive) treatment focused on improving and maintaining response inhibition skills can reduce weight-regain after RYGB surgery.

\section{Conclusion}

After RYGB surgery, patients showed increased activation during a food specific go/no-go task to high-energy food cues in prefrontal brain regions implicated in inhibition. These neural changes after surgery indicate improved response inhibition towards high-energy food cues and increased influence of satiety on processing of low-energy food cues. We found altered neural responses during response inhibition towards both high- and low-energy food cues in reward- 
related areas, which indicate a more positive attitude towards these cues after RYGB. It is plausible that changes in the (re)activity of neural circuits involved in inhibitory control, satiety and reward processing together underlie effective weight-loss by contributing to the shift in preference and intake from high- to low-energy dense foods observed after RYGB. Future research should aim to clarify the association between the neural changes we found and actual measures of eating behaviour and put effort into improving effectivity of weight-loss treatment.

\section{Acknowledgements}

This research was funded by Graduate School VLAG and NWO (The Netherlands Organization for Scientific Research), Veni grant nr. 451-11-021, awarded to SB. Further financial support was provided by Graduate School VLAG. The use of the 3T fMRI facility has been made possible by CAT-AgroFood (Shared Research Facilities Wageningen UR). We thank Lieke van Genderen, Louise Mulder, Monique Zwinkels and Elbrich Postma for their help during data acquisition, and Els Siebelink, Jose Bluemer and Annemarie Kruitwagen for their help in composing the standardized meals. 
Supplementary Table 7.1. Composition of the standardized meal.

\begin{tabular}{l|cc|cc}
\hline & \multicolumn{2}{c|}{ Men } & \multicolumn{2}{c}{ Women } \\
\cline { 2 - 5 } & Pre & Post & Pre & Post \\
\hline Bread Roll ( Wheat bread $( \pm 22 \mathrm{~g} /$ roll $))$ & $4 \mathrm{pcs}$ & $2 \mathrm{pcs}$ & $3 \mathrm{pcs}$ & $1 \mathrm{pcs}$ \\
Margarine (Low-fat) & $30 \mathrm{~g}$ & $15 \mathrm{~g}$ & $15 \mathrm{~g}$ & $15 \mathrm{~g}$ \\
Cheese (Full-fat semi-cured) & $40 \mathrm{~g}$ & $20 \mathrm{~g}$ & $40 \mathrm{~g}$ & $20 \mathrm{~g}$ \\
Ham & $40 \mathrm{~g}$ & $20 \mathrm{~g}$ & $20 \mathrm{~g}$ & - \\
Orange Juice & $150 \mathrm{~g}$ & $75 \mathrm{~g}$ & $100 \mathrm{~g}$ & $50 \mathrm{~g}$ \\
\hline kCal total meal & 570 & 174 & 421 & 107 \\
\hline
\end{tabular}

Supplementary Table 7.2. Hunger ratings provided after meal intake and around 50 minutes before the go/no-go task commenced, before and after RYGB surgery.

\begin{tabular}{lccc}
\hline & Pre-surgery & Post-surgery & Significance \\
\hline Hunger & $11 \pm 21$ & $11 \pm 24$ & $p=0.954$ \\
Fullness & $74 \pm 25$ & $67 \pm 35$ & $p=0.335$ \\
Prospective consumption & $25 \pm 26$ & $8 \pm 18$ & $p=0.051$ \\
Desire to eat & $18 \pm 19$ & $12 \pm 23$ & $p=0.435$ \\
Thirst & $66 \pm 27$ & $53 \pm 29$ & $p=0.049$ \\
\hline
\end{tabular}

Ratings performed on a 100 point VAS. Values are means \pm SD 


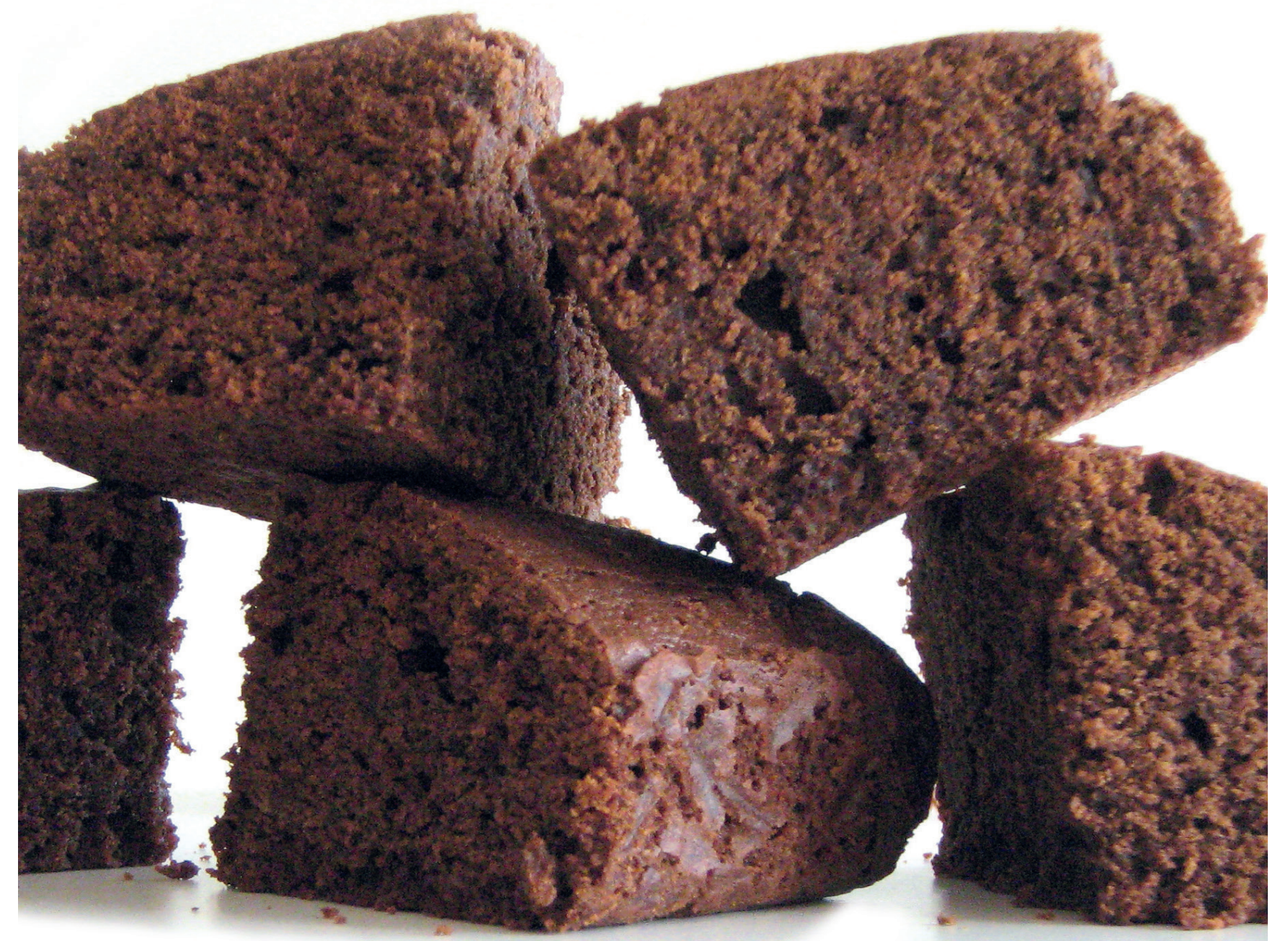




\section{CHAPTER 8}

GENERAL DISCUSSION 
Food reward is a major driver of food intake. Consuming food for pleasure, mostly referred to as hedonic eating, can trigger overeating and thus contribute to obesity. Food reward starts at the sight or smell of food and fluctuates during the food intake cycle, which is often divided into an anticipatory, consummatory, and post-ingestive phase (Kringelbach et al., 2012). The studies described in this thesis focus on behavioural, sensory, physiological and neurophysiological reward responses across the food intake cycle. Figure 8.1, already introduced in chapter 1, provides a schematic overview of this cycle. The topics of the different chapters are also indicated in the figure. This general discussion starts with an overview of the main findings, which is followed by their interpretation in view of other findings. Subsequently, methodological considerations and translational issues are addressed. This discussion ends with suggestions for future research and main conclusions.

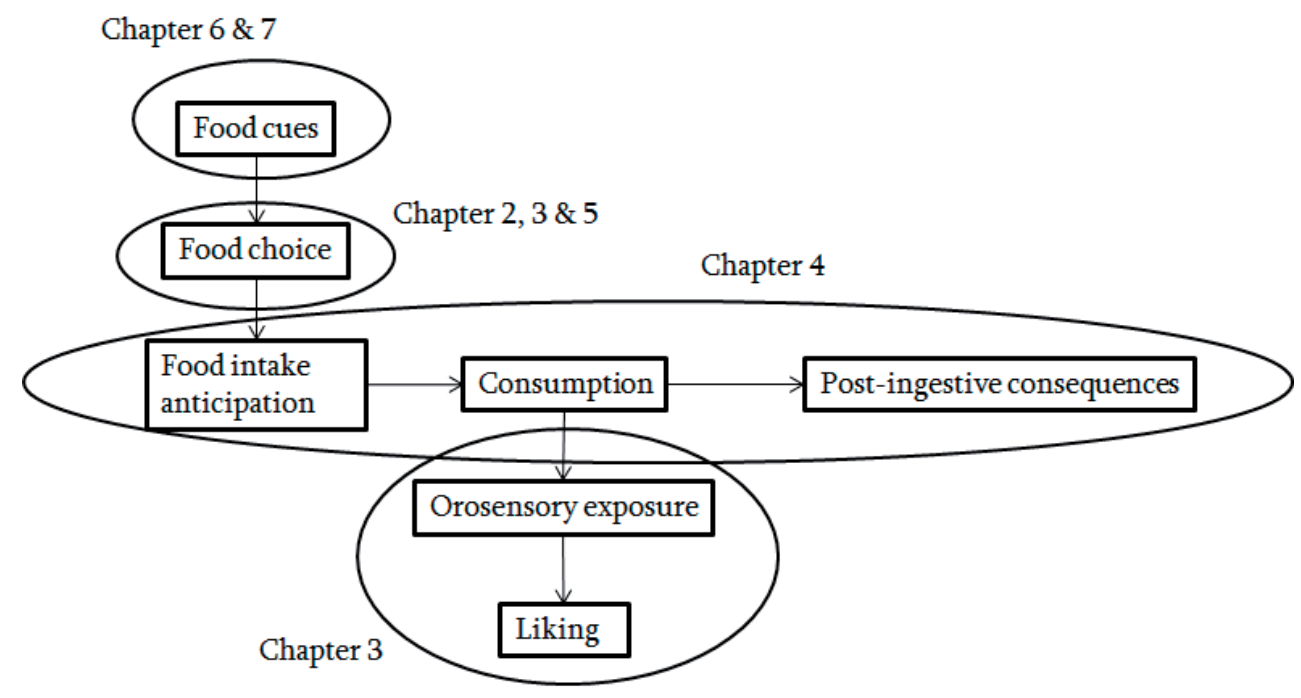

Figure 8.2. Schematic overview of the food intake cycle (Based on Blundell et al., 2010; Kringelbach et al., 2012).

\section{Main findings}

To approach food reward from a behavioural perspective, food preference was used as main outcome measure in this thesis. To be able to measure preferences for four macronutrient and two taste categories, a new method had to be developed. This Macronutrient and Taste Preference Ranking Task (MTPRT) has been described and applied in chapter 2. It was found that food preferences as assessed with this method were highly correlated over two test sessions in a hungry condition. This shows that the MTPRT has good test-retest reliability. In addition, the MTPRT was found to be able to discriminate between different conditions, as shown by successful discrimination of sensory-specific satiety. After consumption of a sweet meal, preference for sweet foods decreased, and after consumption of a savoury meal preference for sweet foods increased. These results indicate that the MTPRT is a valid method to detect changes in food preferences. 
The sensory and physiological perspective of food reward was addressed by studying the role of the endocannabinoid system (ECS). The ECS is a neuromodulatory system that plays in important role in food reward. We hypothesized that modulation of the ECS would alter food preferences and affect sensory perception with regard to sweet taste intensity perception (psychophysics) and liking (psychohedonics). To this end, we used cannabis preparations, and administered by inhalation low doses of $\Delta 9$-tetrahydrocannabinol (THC), cannabidiol (CBD), and placebo, respectively. In contrast to our hypothesis, we found that perceived sweetness intensity and liking did not differ between the THC, CBD and placebo conditions. These results suggest that modulation of the ECS with low doses of THC and CBD does not affect sweet taste intensity perception and liking in humans. Aiming to investigate the connection between the ECS and food reward from an opposite perspective, we set out to study the effect of food taste liking on the ECS. In chapter 4, participants were exposed to a palatable and a neutral food, but they did not know palatability of the food until their first bite. This enabled us to study the effect of food taste liking on physiological responses to food intake, and eliminate effects of prior expectations about food palatability. However, with the experimental set-up used endocannabinoid, ghrelin and pancreatic polypeptide plasma concentrations did not differ between palatable and neutral food intake across anticipation, consumption and post-ingestion. These results suggest that, in the absence of prior expectations about food palatability, food taste liking does not affect endocannabinoid plasma concentrations.

The studies with patients who underwent Roux-en-Y gastric bypass (RYGB) surgery were intended to gain more insight into alterations in food reward. Building on previous reports, we hypothesized that food preferences would change after RYGB, and that this would correlate with changes in brain responses to food cues. Indeed, at two months and one year after RYGB surgery, preferences for high-carbohydrate and high-fat foods were relatively decreased compared to the situation before RYGB, whereas preference for low-energy foods was increased (chapter 5). Possible mechanisms behind these changes in food preferences include alterations in brain response to cues of high-energy and low-energy foods, and shifts in appetite-related hormone levels.

In chapter 6, brain responses to pictures and odours of high-energy and low-energy foods were compared before and at two months after RYGB. We showed that pictures of low-energy foods induced less activation in the left superior frontal gyrus two months after RYGB surgery. Moreover, the difference in activation between pictures of high- and low-energy foods in the left precuneus was smaller. The smaller this difference in activation observed, the larger the decrease in preference for high-energy relative to low-energy foods. For low-energy food odours, we found activation in both the left and the right precuneus after surgery, while the right precuneus was deactivated in response to those odours before surgery. In addition, high-energy food odours induced less deactivation in the right precuneus and the left superior parietal lobule after surgery. A more pronounced reduction of deactivation in this latter area was correlated with a greater increase in preference for low-energy foods. Together, these results point to a role for changes in brain responses to food cues in changed food preferences after RYGB surgery.

With regard to appetite-related hormones and other signalling molecules, we found that plasma concentrations of the endocannabinoid anandamide were increased following RYGB surgery. Plasma concentrations of other endocannabinoids and ghrelin did not change. Moreover, 
neural changes did not correlate with changes in endocannabinoids or ghrelin plasma concentrations. Thus, in our study we did not find evidence for a role of endocannabinoids and ghrelin in changed brain responses to food cues.

To further study brain responses to food cues, changes in brain activation in response to inhibitory responses to high- and low-energy food pictures were studied in chapter 7. After RYGB surgery, activation in reward-related brain areas was decreased in response to both high- and lowenergy food pictures. Prefrontal brain areas were more activated in response to high-energy pictures, which might indicate increased inhibitory control.

\section{Discussion and interpretation}

The aim of this thesis was to add on to the existing knowledge on food reward. To this end, the phenomenon was approached from a behavioural and a (neuro)physiological perspective in healthy individuals and in gastric bypass populations. In this section of the thesis, the different results obtained will be interpreted and discussed. First, the behavioural perspective will be discussed with a focus on the main outcome measure used here, which was food preferences. Next, the learnings about the role of ECS in food reward in humans are discussed. To this end, effects of the pharmacological challenge with the cannabis preparations on sweet taste perception and food preferences will be considered first and then the physiological responses to food intake will be discussed. This is followed by a discussion of changed food preferences and underlying mechanisms following RYGB surgery. In this last section, the neurophysiological perspective is included as well.

\section{Liking ratings and desire to eat rankings}

The behavioural perspective of this thesis focussed mainly on food preferences. To be able to measure relative food preferences for four macronutrient and two taste categories, the MTPRT was designed. The MTPRT consists of a liking part, in which liking of the different foods is rated, and a ranking part in which arrays of four foods are presented and participants are asked to rank those foods on how much they desire to eat the food at that moment. This combination of liking ratings and desire to eat rankings is an important strength of the MTPRT, and makes that the MTPRT provides more information on food preferences than liking ratings or desire to eat rankings alone would. The ranking scores provide insight in behavioural outcomes of changed liking or changed preference for a specific food category (Finlayson et al., 2008). In the MTPRT, the rankings are used to calculate preference scores as explained in chapter 2. The rankings, and thus the preference scores, depend on each other in the sense that they are relative scores. If for example preference for high-carbohydrate foods increases, preference for high-fat, high-protein and/or lowenergy foods has to decrease. Similarly, preference for sweet foods and for savoury foods are each other's opposites. Individual liking ratings, on the other hand, are independent of each other and changes in liking ratings for one food category do not have to result in changes for other food categories. If for example liking of high-carbohydrate foods increases, liking of high-fat, highprotein and low-energy foods can remain unchanged. Individual ratings, however, can be used to gain more insight into the reason behind changed preference scores. An example of this can be found in chapter 5 of this thesis. In this chapter we showed that preference for high-carbohydrate 
and high-fat foods was decreased at two months and one year after RYGB surgery. Moreover, preference for low-energy foods increased after RYGB. The changes in liking ratings for the different food categories provided more insight into these changes. Similar to the rankings, liking of high-carbohydrate and high-fat foods decreased after RYGB surgery, but changes in liking of low-energy foods were minimal. The changes in liking ratings suggest that the decreased preference for high-carbohydrate and high-fat foods leads to an increased preference for low-energy foods. This might explain why some, but not all, studies found increased preference for fruits and vegetables after RYGB surgery (Laurenius et al., 2013; Mathes and Spector, 2012; Ullrich et al., 2013).

\section{Effects of endocannabinoid modulation on food reward in humans}

As described in the introduction, the ECS plays an important role in food reward (Jager and Witkamp, 2014; Piazza et al., 2017), but most evidence for this notion comes from studies in rodents. These studies suggest a role for the ECS in food liking, specifically for sweet taste (De Luca et al., 2012; Higgs et al., 2003). Moreover, endocannabinoids were found to selectively modulate sweet taste responses (Yoshida et al., 2010). In humans, only one study assessed the effects of oral THC on perception of sweet, salty, sour and bitter taste, and this study found no effect (Mattes et al., 1994b). However, Cannabis (i.e. preparations from the Cannabis (spp.) plant) use was found to increase intake of sweet snacks (Foltin et al., 1988), which suggests an effect of Cannabis on sweet taste liking. Therefore, in chapter 3 Cannabis providing a low dose of $\Delta 9$-tetrahydrocannabinol (THC) or cannabidiol (CBD), and a placebo Cannabis were administered to healthy volunteers and effects of these pharmacological challenges on sweet taste intensity perception (psychophysics) and liking (phychohedonics), and on food preferences were assessed. In our study, we found no effect of low doses of THC or CBD on sweet taste intensity perception and liking in humans. Moreover, food preferences as assessed with the MTPRT did not differ between the THC, CBD and placebo condition. However, based on the findings in the MTPRT performed in the same study, one might expect to find effects of THC on preference for fat foods in humans. In the THC condition, preference for high-fat food was 2.6 (scale 1-4), compared with 2.4 in both the CBD and placebo condition, a difference of 0.2 . Although this difference was not statistically significant, the effect size, partial $\eta^{2}=0.23$, can be considered as rather large (Richardson, 2011). We think the difference reflects a meaningful increase in preference for fat foods in the THC condition that may become significant with an increased sample size. In the other two chapters of this thesis that used the MTPRT (chapter 2 and 5) and that included larger sample sizes, smaller differences in relative preference scores (ca 0.15) were found to be statistically significant and considered meaningful. Evidence for the role of the ECS in preference for fat foods comes from a food intake survey in Cannabis users that showed increased consumption of meat, cheese and salty snacks in users compared with non-users (Smit and Crespo, 2001). This points to a modulated preference for savoury high-fat foods. Importantly, evidence from animal studies also suggests a prominent role of the ECS in preference for fat (Di Marzo, 2011; DiPatrizio et al., 2011; DiPatrizio et al., 2013). Therefore, studies with larger sample sizes are merited, focussing on the role of the ECS in preference for fat foods in humans. 


\section{Endocannabinoid responses across the food intake cycle}

Next to the study into effects of ECS modulation on food reward, we also studied the effects of palatable versus neutral food intake on endocannabinoid plasma concentrations. At the start of this thesis there was one published study that suggested that anticipation of palatable food intake induces a response of the ECS (Monteleone et al., 2012). In chapter 4 of this thesis, it was investigated whether consumption of palatable food also triggers an ECS response when food palatability is unknown until the first bite. By keeping the participants unaware of food palatability, the effects of expectations were eliminated. In addition to investigating effects on the ECS, physiological responses across the food intake cycle were studied in this chapter. Whereas exposure to the food through sight and smell led to increased hunger levels and increased appetite for something sweet, this did not induce a physiological response; endocannabinoid, ghrelin and PP plasma concentrations did not change in response to the sight and smell of the food. In addition, palatable versus neutral food consumption did not results in differences in the endocannabinoid response. Together, these results suggest that expectations about food palatability are necessary to increase ghrelin and endocannabinoid release in response to palatable food exposure, as was found in other studies (Monteleone et al., 2012; Rigamonti et al., 2015).

Our results do suggest an orexigenic role for endocannabinoids, the response comparable with that of ghrelin. After food intake, endocannabinoid plasma concentrations decreased (chapter 4). This is in line with a previous study that showed a pre-prandial peak in anandamide concentrations and a drop after food intake (Gatta-Cherifi et al., 2012). More support comes from results of a rat study that showed increased endocannabinoid concentrations in brains of fooddeprived rats, and decreased concentrations during feeding (Kirkham et al., 2002). Our study design, in which the first blood sample was taken five minutes before food intake, was less suitable to investigate pre-prandial changes in endocannabinoid and ghrelin plasma concentrations. Future studies could look into this, for example by taking additional blood samples one hour and thirty minutes before food intake.

\section{Food preferences after RYGB-induced weight loss}

To gain more insight into alterations in food reward, chapters 5, 6 and 7 of this thesis focussed on changes in food preferences and food reward after RYGB surgery. First, in chapter 5 food preferences were assessed before, and two and one year after RYGB surgery. It was shown that rankings of foods high in carbohydrate and fat became lower, indicating decreased preference for these foods after RYGB surgery. In addition, low-energy foods were ranked higher, reflecting higher preference following RYGB surgery. Liking ratings decreased for the high-carbohydrate and highfat foods, but did only marginally increase for the low-energy foods, which makes it likely that the increased preference for the low-energy foods is relative and a consequence of decreased desire to eat foods high in carbohydrate or fat. These results are in line with previous studies that showed decreased desire to eat high-energy foods with no change for low-energy foods (Miras et al., 2012; Ochner et al., 2012b). These results suggest that a shift towards a healthier food intake behaviour is mainly driven by a lower preference for high-energy foods and not by increased liking of lowenergy fruits and vegetables. 


\section{Mechanisms behind changed food preferences after RYGB-induced weight loss}

To explore potential mechanisms behind the changed food preferences found in chapter 5 , we compared brain responses to food cues of high-energy and low-energy foods, and endocannabinoid and ghrelin plasma concentrations, before and after RYGB surgery in chapter 6 , and response inhibition-related brain activation in chapter 7. In chapter 6 we compared neural responses to odours and pictures before surgery with those two months after RYGB, when participants were in a satiated state. Furthermore, we investigated differences in endocannabinoid and ghrelin plasma concentrations after a three hour fast. The changes in neural responses to food cues were found to be primarily located in a frontoparietal network, which is involved in cognitive control during (food) cue processing. Importantly, we did not find changes in activation in the mesolimbic system as other studies found (see e.g. Faulconbridge et al., 2016; Ochner et al., 2012b; Scholtz et al., 2014). A possible explanation for this is that our participants were scanned in a satiated state, whereas in other studies participants were hungry. A previous study that scanned participants in a satiated state did not find significant changes in brain activation from before to after RYGB surgery, possibly due to the small sample size (Ochner et al., 2012a). Together, these results suggest that changes in neural responses to food cues after RYGB surgery are more prominent when individuals are hungry. The changes in neural responses to food cues might facilitate selection of healthier foods (Ochner et al., 2012b).

With respect to the endocannabinoids and ghrelin, anandamide concentrations were increased two months after RYGB surgery compared to those before surgery. This change did not correlate with neural changes, which might be a suggestion that the ECS does not play a role in changed neural responses to food cues, and is possibly not involved in altered food choice behaviour. However, more studies are necessary to be able to draw firm conclusions. An important limitation is also that (changes in) plasma endocannabinoid levels do not necessarily reflect events taking place in the brain. We did find correlations between differences in food preferences and neural changes, which strengthens the evidence that alterations in food preferences from before to after RYGB surgery are related to changes in neural processing of food cues.

An important brain area that was found in our study in chapter 6 (neural responses to food cues) is the precuneus, a brain area previously implicated in decreased liking of high-energy foods after RYGB (Ochner et al., 2012b). In a previous study that compared neural responses to food cues in RYGB patients at least six months after surgery with neural responses in an obese population not undergoing surgery, precuneus activation was found to be higher in the nonsurgical group (Frank et al., 2016). In addition, resting state activity in the precuneus has been shown to be greater in obese versus lean individuals (Zhang et al., 2015), and to be negatively correlated with obesity preventive eating tendency (Nakamura and Ikuta, 2017). Taken together, the changes in precuneus activation from before to after RYGB surgery might relate to better impulse control and be an important factor in the apparently healthier food intake behaviour after RYGB surgery.

In chapter 7 neural changes to inhibition were assessed with a food-specific go/no-go task, in which participants were asked to respond to one set of food stimuli and to withhold response to another set of food stimuli. Our behavioural data in the go/no-go task showed that participants made relatively few mistakes before surgery, resulting in a ceiling effect with no room for significant improvement. A more difficult task could have provided more insight into functional outcomes of 
the neural changes that we found. A previous study that compared good and bad responders to RYGB at 9-15 years after RYGB surgery showed that the bad responders performed worse in foodrelated paradigms that require impulse control (Hogenkamp et al., 2015). Moreover, better inhibition was shown to be related to increased weight loss at six months after RYGB surgery (Kulendran et al., 2017). Taken together, being able to resist impulses and execute response inhibition are factors in successful weight management after RYGB surgery.

\section{Methodological considerations}

In this section some methodological considerations regarding the studies described in this thesis are discussed. First, the relevance of food preferences as measured with the MTPRT is discussed. Then considerations regarding the study populations are elaborated upon; sex differences in the effects of the Cannabis challenge on sweet taste perception, and the study population in the RYGB-studies are discussed. Finally, considerations regarding hunger state in the study of food reward are discussed.

\section{Macronutrient and taste preference ranking task}

Measurement of food preferences instead of actual food choice. In the MTPRT, participants rank food products that differ in macronutrient content (carbohydrate, fat, protein and low-energy) and taste (sweet and savoury) according to what they desire to eat at that moment. The rankings are then used to calculate preference scores for the different macronutrient and taste categories. The MTPRT has been shown to be a reliable and valid method to assess food preference in a behavioural experiment, but it is important to note that food choices as made in the MTPRT have not yet been compared with actual food choices. It therefore remains to be determined to what extent the MTPRT reflects actual behaviour. Based on previous studies that found strong correlations between choices in a forced-choice procedure and actual intake (Griffioen-Roose et al., 2010; Griffioen-Roose et al., 2011), we expect that rankings in the MTRPT correlate with actual food intake behaviour. Importantly, the MTPRT is the first food preference task that uses a ranking procedure to assess food preferences for four macronutrient and two taste categories. As for the studies described in chapter 3 (effect of cannabis challenge on food preferences) and chapter 5 (food preferences after RYGB surgery) we were specifically interested to include a broad range of food categories. Therefore, we considered the MTPRT to be the most suitable method to measure food preferences.

\section{Study population}

Males versus females. The study that focused on the role of the ECS in sweet taste perception (chapter 3 ) included only male participants. It has been shown that endocannabinoid pharmacology differs between males and females (Craft et al., 2013; Diaz et al., 2009), with males being more susceptible to increasing their food intake, and females being more responsive to feelings of depression and anxiety (Fattore and Fratta, 2010). In animals, males were found to be more sensitive to the appetite-modulation properties of cannabinoid administration than females (Diaz et al., 2009). If there would be an effect of low doses of THC and CBD on sweet taste perception, we would have expected to find this effect in our male study population. 
Gastric bypass patients. The studies in gastric bypass patients (chapters 5, 6 and 7) did not include a control group that underwent another type of gastric bypass like vertical sleeve gastrectomy, or was exposed to a dietary weight-loss intervention. It is therefore not possible to say whether the changes in food preference and neural processing of food cues we found are specific for the RYGB patient group, or whether similar changes occur after other types of gastric bypass surgery or behavioural weight loss methods. The within-subjects design enabled us to gain more insight into alterations in food reward after weight loss and strengthened the evidence for the notion that altered food preferences after RYGB are related to changes in neural responses to food cues.

\section{Hunger state}

It is important to consider hunger state in the study of (palatable) food intake and food reward. Even though so-called homeostatic eating has a hedonic component, it has been argued that participants should feel satiated to be able to study hedonic eating (Monteleone et al., 2012). That is the reason why, in both chapter 4 and chapter 6 , we provided participants with a standardized meal to satiate them before studying palatable versus neutral food intake, and food reward. In chapter 4, this was performed following the design of Monteleone and colleagues (2012), and other studies have followed this design (Monteleone et al., 2016; Rigamonti et al., 2015). Participants were provided with a standard breakfast before they were asked to consume the palatable or the neutral food.

The inclusion of satiated participants is less common in the study of (changes in) food reward in RYGB patients. Most other studies that assessed changes in food reward processing in gastric bypass patients scanned participants in a hungry state (see e.g. Faulconbridge et al., 2016; Ochner et al., 2012b; Scholtz et al., 2014), whereas in our study (chapter 6), participants were satiated. To our knowledge, one other study also included participants in a fed state, but this study did not find any differences when comparing pre- to postsurgery. This is possibly due to the small sample size ( $n=5)$ (Ochner et al., 2012a). The comparison of our results with results described in literature suggests that changes in brain reward responses from before to after RYGB surgery depend on hunger state. More studies that include participants in a satiated state or that compare neural responses of participants in a hungry versus a satiated state are needed to obtain more conclusive insights on these differences and their relation with changes in food preferences.

\section{Translational issues}

As mentioned before in this general discussion, most evidence for the role of the ECS in food reward comes from animal studies. In this thesis, it was tried to translate some of these findings to the human situation. Some issues related to translating methodology from animal to human studies are discussed in the following section. First, the use of pharmacological challenges to modulate the ECS in human studies is elaborated upon, followed by discussion of the relevance of plasma endocannabinoid concentrations. 


\section{Pharmacological challenge}

Modulating the ECS with pharmacological compounds is a challenge in humans, especially when it comes to inhibiting the ECS. Different compounds are available for use in animal studies, e.g., AM 251 and rimonabant (Pertwee, 2006), but these are not approved for use in humans. In this thesis, cannabidiol (CBD) was used aiming to inhibit the ECS. However, the pharmacodynamic properties of CBD are not completely understood (Ligresti et al., 2016; McPartland et al., 2015; Pertwee, 2008). CBD displays a wide spectrum of activities on various molecular targets and produces multiple pharmacological effects. It is therefore considered to be more of a multiple-target molecule than a classical cannabinoid (Ligresti et al., 2016; Pertwee, 2008). However, CBD has been suggested to antagonize the ECS via $\mathrm{CB}_{1}$ (Pertwee, 2008) and some animal studies, but not all, found decreased food intake upon CBD administration (Farrimond et al., 2012; Ligresti et al., 2016; Wierucka-Rybak et al., 2014; Wiley et al., 2005). The design of the study described in chapter 3 (cannabis challenge) benefited from administering not only an agonist, THC, but also a potential antagonist. In this way, the effects of ECS activation and inhibition could be studied and this might provide more insight into the role of the ECS in sweet taste intensity perception and liking than ECS activation alone. As options for $\mathrm{CB}_{1}$ antagonists for human use are limited, we decided to include CBD.

Another issue to consider it the use of Cannabis plant material, which is a mixture of compounds (McPartland et al., 2015), compared with pure compounds. With a pure compound, it is clear what is administered allowing to directly link this to the effects observed. Using a mixture of compounds, this is not so clear. However, the placebo Cannabis that was used in chapter 3 of this thesis had identical moisture and terpenoid profile to the active Cannabis, and thus taste and smell experiences were similar (Kowal et al., 2015). Thus, the only difference between active and placebo Cannabis was the presence of the active compound under study. Moreover, we showed successful blinding, as only one out of the ten participants could convincingly and correctly identify the THC condition. If we would have used pure THC, this number would most likely have been higher (Van Hell et al., 2011).

\section{What is the relevance of plasma endocannabinoids?}

Evidence from animal studies suggests that endocannabinoid activity in different brain areas relates to food intake behaviour (see e.g. Kirkham et al., 2002) and that the ECS in the gut plays a role in fat intake (see e.g. DiPatrizio et al., 2011). To translate these findings to humans is complicated. In human studies, often plasma or serum samples are used to assess endocannabinoid concentrations. However, the source and role of blood endocannabinoids are at present unclear. It has been suggested that endocannabinoids in the blood stream are the result of spill-over from other organs, like the brain and gut, but further research is necessary to gain more insight into this. One important factor to consider is that endocannabinoids are formed and released on demand, rather than stored in vesicles in the cell (Di Marzo et al., 2009). This suggests that increased plasma concentrations reflect increased endocannabinoid formation but delay-effects and degradation may play a role.

Even though we do not know the exact source and role of blood endocannabinoids, they have been found to be related to weight status (Engeli et al., 2005), and olfactory function (Pastor 
et al., 2016), and to respond to challenges like food intake (Monteleone et al., 2012) and negative mood induction (Schrieks et al., 2015). These findings suggest that blood endocannabinoids are a relevant source of information about functioning of the endocannabinoid system, and in humans they are the best option to study physiological responses of the ECS.

\section{Suggestions for future research}

The results of this thesis answered questions regarding food reward, but they also gave rise to new questions. Therefore, suggestions for future research are discussed in the following section. As for the 'discussion and interpretation'-section, this section will follow the line of the chapters. First, food preferences are discussed, followed by the role of the ECS in food reward. Finally, suggestions for research into obesity and weight-loss interventions such as RYGB are provided.

\section{Measurements of food preferences: predictor of food choice?}

As mentioned in the methodological considerations, it is unknown to what extent food preferences as measured with the MTPRT relate to actual food choice. To be able to use the MTPRT as a predictor of actual eating behaviour, it should first be determined whether food preference scores as assessed with the MTPRT indeed predict actual food choice, for example in a buffet setting. It would be of interest to compare different experimental conditions, by including differences in hunger state (hungry versus satiated) or taste of a previous meal (sweet or savoury). By including these different conditions, it is not only possible to relate food choice in the MTPRT to actual food choice, but changes in food preference scores as assessed with the MTPRT can also be related to changes in actual food choice.

\section{Endocannabinoid responses to food exposure during the day}

Over the past years, it has been established that anticipation of palatable food intake induced a rise in endocannabinoid plasma concentrations (Monteleone et al., 2012; Rigamonti et al., 2015). In chapter 4, we showed that endocannabinoid plasma concentrations decrease after food intake. Previously, it has been shown that endocannabinoids follow a circadian rhythm (Vaughn et al., 2010). For example, 2-AG concentrations increase throughout the morning, reaching a maximum early afternoon, and decrease during the late afternoon and evening, to a minimum during the night (Hanlon et al., 2015). This pattern was not disturbed by food intake. Importantly, participants received standardized meals that were not highly palatable. In other studies, palatable foods were shown to increase 2-AG concentrations (Monteleone et al., 2012; Rigamonti et al., 2015). It would therefore be interesting to assess the effect of palatable food intake on circadian endocannabinoid patterns. As exposure to palatable foods modulates endocannabinoid concentrations, we would expect that it also modulates the circadian pattern of endocannabinoids, possibly by increasing endocannabinoid concentrations. Increased endocannabinoid concentrations might in turn increase food intake and lead to overeating. It would be interesting for future studies to relate (changes in) endocannabinoid concentrations to feelings of hunger and food intake, and possibly to overeating and obesity. 


\section{The hyperactive endocannabinoid system in obesity}

Animal studies have provided evidence for increased endocannabinoid concentrations in response to food. Orosensory exposure to fat increases anandamide and 2-AG concentrations in the rat jejunum (DiPatrizio et al., 2011; DiPatrizio et al., 2013), and infusion of a $\mathrm{CB}_{1}$ antagonist in the intestine decreased ad libitum sham intake of fat (DiPatrizio et al., 2011) and reduced preference for specific fatty acids (DiPatrizio et al., 2013). Similarly, a high-fat diet increases anandamide and 2AG concentrations in plasma and jejunum in mice. Moreover a high-fat diet induces overeating and body weight gain in these mice, possibly through overactivation of the ECS (Argueta and DiPatrizio, 2017).

Higher endocannabinoid concentrations have been found in obese compared with lean individuals (Bluher et al., 2006; Engeli et al., 2005), but is unknown whether this is a cause or an effect of obesity. It is also unknown what the functional consequences of these increased endocannabinoid concentrations are. Ideally, long-term follow-up of a large cohort could provide more insight into these questions, but that is an expensive and time-consuming method. In a more realisitic study design, changes in endocannabinoid tone during weight gain could be studied in an animal model. Furthermore, differences in endocannabinoid responses across the food intake cycle between lean, overweight and obese individuals can be studied.

\section{The endocannabinoid system after weight loss}

As mentioned before, it is not known whether increased endocannabinoid tone in obesity is a cause or an effect. Similarly, the number of studies that assessed the effect of weight loss on endocannabinoid tone is limited and findings are contradicting. In chapter 5 of this thesis, we have shown increased anandamide concentrations two months after RYGB surgery, which corresponds to ca $10 \%$ weight loss. Other studies showed decreased anandamide and PEA concentrations one year after RYGB (Montecucco et al., 2015) or no change in endocannabinoid concentrations at six months or two years after RYGB (Mallipedhi et al., 2015; Quercioli et al., 2013). Importantly, contradicting results have also been found in weight loss trials, with some studies that did not find differences in endocannabinoid concentrations (Engeli et al., 2005; Engeli et al., 2008), and others that found decreased 2-AG and anandamide concentrations (Di Marzo et al., 2008) or increased PEA concentrations (Abdulnour et al., 2014). These discrepancies can be related to differences in the duration of the weight loss intervention, which also relates to how fast weight is lost over time, and warrant further investigations.

\section{Weight loss through RYGB versus dietary intervention}

As mentioned in the methodological considerations, our studies in RYGB did not have a control group. For future studies it would be advisable to compare weight loss after RYGB surgery with behavioural weight loss methods or other types of weight loss surgeries, as some studies have also done (Bruce et al., 2014; Faulconbridge et al., 2016; Primeaux et al., 2015). This will help identify changes in food reward specifically related to RYGB, and not to other surgical or behavioural weight loss interventions. As RYGB is the most successful method to lose weight (Buchwald et al., 2004; Gloy et al., 2013), these differences might guide the development of a successful non-surgical weight loss intervention. 
Another point of interest is time of follow-up. Meta-analyses of weight loss outcomes of RYGB indicate that patients start to regain some weight after eighteen to twenty-four months (Buchwald et al., 2004; Gloy et al., 2013). In this thesis, food preferences were assessed up to twelve months and changes in neural responses to food cues was only assessed at two months after RYG surgery. It would be interesting to include a longer follow-up as to be able to identify potential reversal of food preferences or food reward processing to the before-surgery situation and a possible relation between reversal and weight gain.

\section{Conclusions}

Modulation of the endocannabinoid system with low doses of THC and CBD does not influence sweet taste intensity perception and liking and food preferences. Vice versa, food palatability in the absence of expectations does not affect endocannabinoid responses to food intake.

RYGB surgery induces a decreased preference for high-carbohydrate and high-fat foods, and increased preference for low-energy foods, which is related to altered brain reward processing, but not to changes in endocannabinoid tone. The success of RYGB surgery and the changes in food choice might in part be caused by an improved inhibitory response to high-energy foods. 


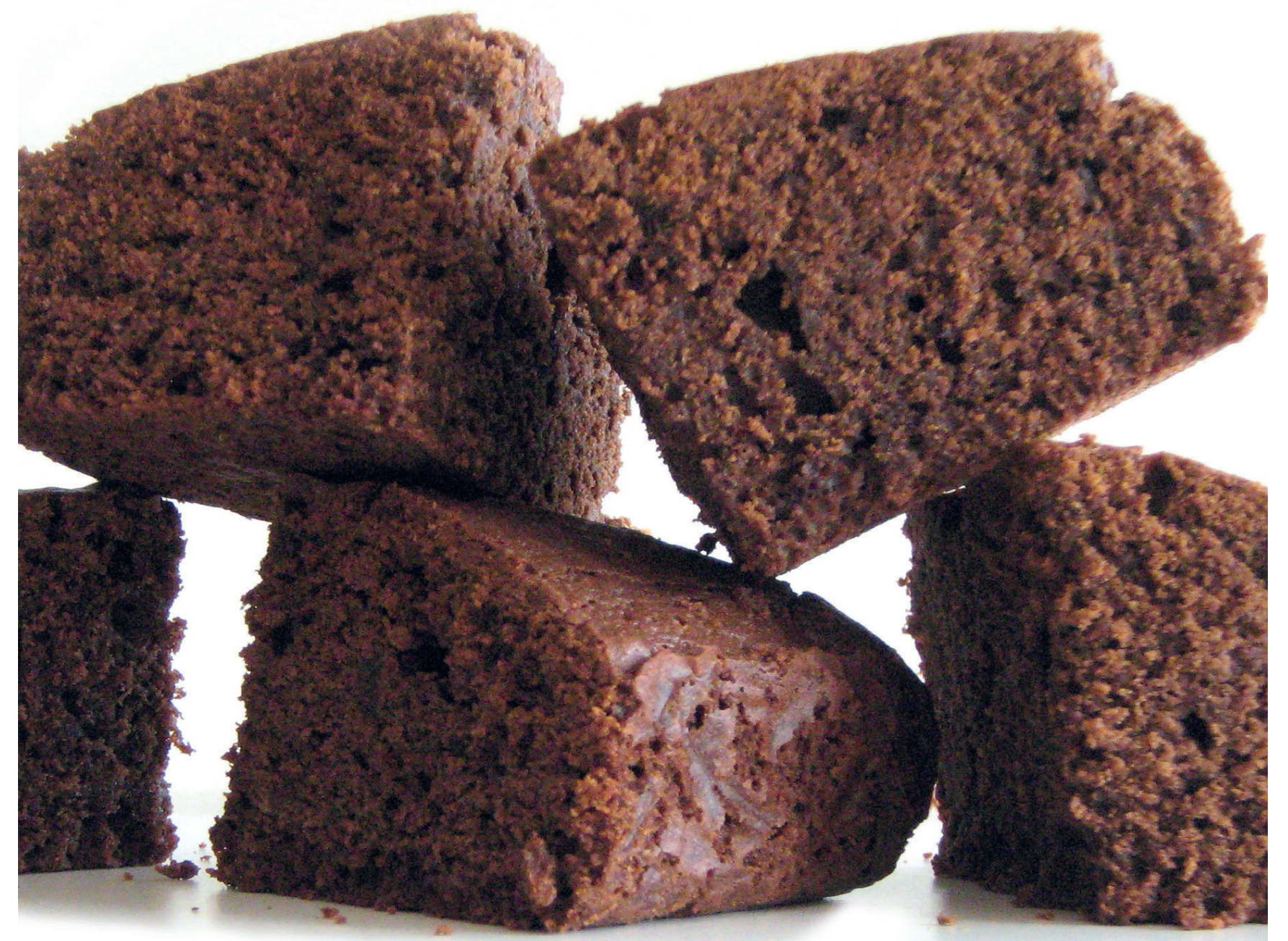


REFERENCES 
Abdulnour, J., Yasari, S., Rabasa-Lhoret, R., Faraj, M., Petrosino, S., Piscitelli, F., Prud' homme, D., Di Marzo, V., 2014. Circulating endocannabinoids in insulin sensitive vs. Insulin resistant obese postmenopausal women. A monet group study. Obesity 22, 211-216.

Abel, E.L., 1975. Cannabis: Effects on hunger and thirst. Behavioral Biology 15, 255-281.

Argueta, D.A., DiPatrizio, N.V., 2017. Peripheral endocannabinoid signaling controls hyperphagia in western diet-induced obesity. Physiology \& Behavior 171, 32-39.

Asao, K., Luo, W., Herman, W.H., 2012. Reproducibility of the measurement of sweet taste preferences. Appetite 59, 927-932.

Ashburner, J., 2007. A fast diffeomorphic image registration algorithm. Neuroimage 38, 95-113.

Balvers, M.G.J., Verhoeckx, K.C.M., Witkamp, R.F., 2009. Development and validation of a quantitative method for the determination of 12 endocannabinoids and related compounds in human plasma using liquid chromatography-tandem mass spectrometry. Journal of Chromatography B 877, 1583-1590.

Barg, J., Fride, E., Hanus, L., Levy, R., Matus-Leibovitch, N., Heldman, E., Bayewitch, M., Mechoulam, R., Vogel, Z., 1995. Cannabinomimetic behavioral effects of and adenylate cyclase inhibition by two new endogenous anandamides. European Journal of Pharmacology 287, 145-152.

Barkeling, B., Rossner, S., Bjorvell, H., 1990. Effect of a high-protein meal (meat) and a high-carbohydrate meal (vegetarian) on satiety measured by automated computerized monitoring of subsequent food intake, motivation to eat and food preferences. International Journal of Obesity and Related Metabolic Disorders 14, 743-751.

Batterink, L., Yokum, S., Stice, E., 2010. Body mass correlates inversely with inhibitory control in response to food among adolescent girls: An fMRI study. Neuroimage 52, 1696-1703.

Behary, P., Miras, A.D., 2015. Food preferences and underlying mechanisms after bariatric surgery. Proceedings of the Nutrition Society, 1-7.

Bellocchio, L., Lafentre, P., Cannich, A., Cota, D., Puente, N., Grandes, P., Chaouloff, F., Piazza, P.V., Marsicano, G., 2010. Bimodal control of stimulated food intake by the endocannabinoid system. Nature Neuroscience 13, 281-283.

Berridge, K.C., 1996. Food reward: Brain substrates of wanting and liking. Neuroscience and Biobehavioral Reviews 20, 1-25.

Berthoud, H.-R., Zheng, H., Shin, A.C., 2012. Food reward in the obese and after weight loss induced by calorie restriction and bariatric surgery. Annals of the New York Academy of Sciences 1264, 36-48.

Berthoud, H.R., Shin, A.C., Zheng, H., 2011. Obesity surgery and gut-brain communication. Physiology and Behavior 105, 106-119.

Bewick, G.A., 2012. Bowels control brain: Gut hormones and obesity. Biochemia Medica 22, 283-297.

Birch, L.L., Billman, J., Richards, S.S., 1984. Time of day influences food acceptability. Appetite 5, 109-116.

Bjork, J.M., Hommer, D.W., 2007. Anticipating instrumentally obtained and passively-received rewards: A factorial fMRI investigation. Behavioural brain research 177, 165-170.

Bluher, M., Engeli, S., Kloting, N., Berndt, J., Fasshauer, M., Batkai, S., Pacher, P., Schon, M.R., Jordan, J., Stumvoll, M., 2006. Dysregulation of the peripheral and adipose tissue endocannabinoid system in human abdominal obesity. Diabetes 55, 3053-3060.

Blundell, J., De Graaf, C., Hulshof, T., Jebb, S., Livingstone, B., Lluch, A., Mela, D., Salah, S., Schuring, E., Van Der Knaap, H., Westerterp, M., 2010. Appetite control: Methodological aspects of the evaluation of foods. Obesity Reviews 11, 251-270.

Bojsen-Moller, K.N., Jacobsen, S.H., Dirksen, C., Jorgensen, N.B., Reitelseder, S., Jensen, J.E., Kristiansen, V.B., Holst, J.J., van Hall, G., Madsbad, S., 2015. Accelerated protein digestion and amino acid absorption after rouxen-y gastric bypass. The American journal of clinical nutrition 102, 600-607. 
Boltong, A., Aranda, S., Keast, R., Wynne, R., Francis, P.A., Chirgwin, J., Gough, K., 2014. A prospective cohort study of the effects of adjuvant breast cancer chemotherapy on taste function, food liking, appetite and associated nutritional outcomes. PLOS ONE 9 .

Boltong, A., Keast, R., Aranda, S., 2012. Experiences and consequences of altered taste, flavour and food hedonics during chemotherapy treatment. Supportive Care in Cancer 20, 2765-2774.

Bongers, P., van de Giessen, E., Roefs, A., Nederkoorn, C., Booij, J., van den Brink, W., Jansen, A., 2015. Being impulsive and obese increases susceptibility to speeded detection of high-calorie foods. Health psychology 34, 677-685.

Booth, J.R., Burman, D.D., Meyer, J.R., Lei, Z., Trommer, B.L., Davenport, N.D., Li, W., Parrish, T.B., Gitelman, D.R., Mesulam, M.M., 2003. Neural development of selective attention and response inhibition. Neuroimage 20, 737-751.

Borg, C.M., Le Roux, C.W., Ghatei, M.A., Bloom, S.R., Patel, A.G., Aylwin, S.J.B., 2006. Progressive rise in gut hormone levels after roux-en-y gastric bypass suggests gut adaptation and explains altered satiety. British Journal of Surgery 93, 210-215.

Boutelle, K.N., Bouton, M.E., 2015. Implications of learning theory for developing programs to decrease overeating. Appetite 93, 62-74.

Bragulat, V., Dzemidzic, M., Bruno, C., Cox, C.A., Talavage, T., Considine, R.V., Kareken, D.A., 2010. Foodrelated odor probes of brain reward circuits during hunger: A pilot fMRI study. Obesity 18, 1566-1571.

Brisbois-Clarkson, T.D., McIsaac, T.M., Goonewardene, L.A., Wismer, W.V., 2009. Modification and validation of a macronutrient preference checklist for use in north america. Appetite 53, 461-464.

Brisbois, T.D., de Kock, I.H., Watanabe, S.M., Mirhosseini, M., Lamoureux, D.C., Chasen, M., MacDonald, N., Baracos, V.E., Wismer, W.V., 2011. Delta-9-tetrahydrocannabinol may palliate altered chemosensory perception in cancer patients: Results of a randomized, double-blind, placebo-controlled pilot trial. Annals of Oncology 22, 2086-2093.

Bruce, A.S., Bruce, J.M., Ness, A.R., Lepping, R.J., Malley, S., Hancock, L., Powell, J., Patrician, T.M., Breslin, F.J., Martin, L.E., Donnelly, J.E., Brooks, W.M., Savage, C.R., 2014. A comparison of functional brain changes associated with surgical versus behavioral weight loss. Obesity 22, 337-343.

Buchwald, H., Avidor, Y., Braunwald, E., Jensen, M.D., Pories, W., Fahrbach, K., Schoelles, K., 2004. Bariatric surgery: A systematic review and meta-analysis. Journal of the American Medical Association 292, 1724-1737. Bueter, M., Miras, A.D., Chichger, H., Fenske, W., Ghatei, M.A., Bloom, S.R., Unwin, R.J., Lutz, T.A., Spector, A.C., Le Roux, C.W., 2011. Alterations of sucrose preference after roux-en-y gastric bypass. Physiology and Behavior 104, 709-721.

Burgess, B., Rao, S.P., Tepper, B.J., 2016. Changes in liking for sweet and fatty foods following weight loss in women are related to prop phenotype but not to diet. Obesity 24, 1867-1873.

Buss, J., Havel, P.J., Epel, E., Lin, J., Blackburn, E., Daubenmier, J., 2014. Associations of ghrelin with eating behaviors, stress, metabolic factors, and telomere length among overweight and obese women: Preliminary evidence of attenuated ghrelin effects in obesity? Appetite 76, 84-94.

Cameron, J.D., Goldfield, G.S., Cyr, M.-J., Doucet, É., 2008. The effects of prolonged caloric restriction leading to weight-loss on food hedonics and reinforcement. Physiology \& Behavior 94, 474-480.

Carver, C.S., White, T.L., 1994. Behavioral inhibition, behavioral activation, and affective responses to impending reward and punishment: The bis/bas scales. Journal of personality and social psychology 67, 319.

Chambers, A.P., Vemuri, V.K., Peng, Y., Wood, J.T., Olszewska, T., Pittman, Q.J., Makriyannis, A., Sharkey, K.A., 2007. A neutral CB1 receptor antagonist reduces weight gain in rat. American Journal of Physiology Regulatory Integrative and Comparative Physiology 293, R2185-R2193. 
Charbonnier, L., van der Laan, L.N., Viergever, M.A., Smeets, P.A.M., 2015a. Functional mri of challenging food choices: Forced choice between equally liked high- and low-calorie foods in the absence of hunger. PLoS ONE 10, e0131727.

Charbonnier, L., van Meer, F., van der Laan, L.N., Viergever, M.A., Smeets, P.A.M., 2015b. Standardized food images: A photographing protocol and image database. Appetite 96, 166-173.

Chen, Q., Fuentes, L.J., Zhou, X., 2010. Biasing the organism for novelty: A pervasive property of the attention system. Human brain mapping 31, 1146-1156.

Chikazoe, J., Jimura, K., Asari, T., Yamashita, K., Morimoto, H., Hirose, S., Miyashita, Y., Konishi, S., 2009. Functional dissociation in right inferior frontal cortex during performance of go/no-go task. Cerebral cortex 19, 146-152.

Christensen, R., Kristensen, P.K., Bartels, E.M., Bliddal, H., Astrup, A., 2007. Efficacy and safety of the weightloss drug rimonabant: A meta-analysis of randomised trials. The Lancet 370, 1706-1713.

Cojan, Y., Waber, L., Carruzzo, A., Vuilleumier, P., 2009. Motor inhibition in hysterical conversion paralysis. Neuroimage 47, 1026-1037.

Cooper, S.J., 2004. Endocannabinoids and food consumption: Comparisons with benzodiazepine and opioid palatability-dependent appetite. European Journal of Pharmacology 500, 37-49.

Courcoulas, A.P., Christian, N.J., Belle, S.H., Berk, P.D., Flum, D.R., Garcia, L., Horlick, M., Kalarchian, M.A., King, W.C., Mitchell, J.E., Patterson, E.J., Pender, J.R., Pomp, A., Pories, W.J., Thirlby, R.C., Yanovski, S.Z., Wolfe, B.M., 2013. Weight change and health outcomes at three years after bariatric surgery among patients with severe obesity. JAMA 310, 2416-2425.

Craft, R.M., Marusich, J.A., Wiley, J.L., 2013. Sex differences in cannabinoid pharmacology: A reflection of differences in the endocannabinoid system? Life Sciences 92, 476-481.

Crystal, S.R., Teff, K.L., 2006. Tasting fat: Cephalic phase hormonal responses and food intake in restrained and unrestrained eaters. Physiology \& Behavior 89, 213-220.

Cummings, D.E., Frayo, R.S., Marmonier, C., Aubert, R., Chapelot, D., 2004. Plasma ghrelin levels and hunger scores in humans initiating meals voluntarily without time- and food-related cues. American Journal of Physiology - Endocrinology And Metabolism 287, E297-E304.

Cummings, D.E., Purnell, J.Q., Frayo, R.S., Schmidova, K., Wisse, B.E., Weigle, D.S., 2001. A preprandial rise in plasma ghrelin levels suggests a role in meal initiation in humans. Diabetes 50, 1714-1719.

Curioni, C., Andre, C., 2006. Rimonabant for overweight or obesity. The Cochrane database of systematic reviews, Cd006162.

Cushing, C.C., Benoit, S.C., Peugh, J.L., Reiter-Purtill, J., Inge, T.H., Zeller, M.H., 2013. Longitudinal trends in hedonic hunger after roux-en-y gastric bypass in adolescents. Surgery for obesity and related diseases.

Davis, C., Strachan, S., Berkson, M., 2004. Sensitivity to reward: Implications for overeating and overweight. Appetite 42, 131-138.

de Bruijn, S.E.M., de Vries, Y.C., de Graaf, C., Boesveldt, S., Jager, G., 2017. The reliability and validity of the macronutrient and taste preference ranking task: A new method to measure food preferences. Food Quality and Preference 57, 32-40.

De Graaf, C., Jas, P., Van der Kooy, K., Leenen, R., 1993. Circadian rhythms of appetite at different stages of a weight loss programme. International Journal of Obesity and Related Metabolic Disorders 17, 521-526.

de Graaf, C., van Staveren, W., Burema, J., 1996. Psychophysical and psychohedonic functions of four common food flavours in elderly subjects. Chemical senses 21, 293-302.

De Luca, M.A., Solinas, M., Bimpisidis, Z., Goldberg, S.R., Di Chiara, G., 2012. Cannabinoid facilitation of behavioral and biochemical hedonic taste responses. Neuropharmacology 63, 161-168. 
de Vries, Y.C., Helmich, E., Karsten, M.D., Boesveldt, S., Winkels, R.M., van Laarhoven, H.W., 2016. The impact of chemosensory and food-related changes in patients with advanced oesophagogastric cancer treated with capecitabine and oxaliplatin: A qualitative study. Supportive care in cancer 24, 3119-3126.

de Vries, Y.C., Winkels, R.M., Van Den Berg, M.C., De Graaf, C., Kelfkens, C.S., de Kruif, J.T.C.M., Göker, E., Sommeijer, D.W., Grosfeld, S., Van Laarhoven, H.W., Kampman, E., Boesveldt, S., 2017. Altered food preferences and chemosensory perception during chemotherapy in breast cancer patients: A longitudinal comparison with healthy controls. Submitted.

Deichmann, R., Gottfried, J.A., Hutton, C., Turner, R., 2003. Optimized epi for fmri studies of the orbitofrontal cortex. Neuroimage 19, 430-441.

Di Marzo, V., 2011. Endocannabinoids: An appetite for fat. Proceedings of the National Academy of Sciences of the United States of America 108, 12567-12568.

Di Marzo, V., Côté, M., Matias, I., Lemieux, I., Arsenault, B.J., Cartier, A., Piscitelli, F., Petrosino, S., Alméras, N., Després, J.-P., 2008. Changes in plasma endocannabinoid levels in viscerally obese men following a 1 year lifestyle modification programme and waist circumference reduction: Associations with changes in metabolic risk factors. Diabetologia 52, 213.

Di Marzo, V., Ligresti, A., Cristino, L., 2009. The endocannabinoid system as a link between homoestatic and hedonic pathways involved in energy balance regulation. International Journal of Obesity 33, S18-S24.

Diaz, S., Farhang, B., Hoien, J., Stahlman, M., Adatia, N., Cox, J.M., Wagner, E.J., 2009. Sex differences in the cannabinoid modulation of appetite, body temperature and neurotransmission at pomc synapses. Neuroendocrinology 89, 424-440.

Diekhof, E.K., Nerenberg, L., Falkai, P., Dechent, P., Baudewig, J., Gruber, O., 2012. Impulsive personality and the ability to resist immediate reward: An fmri study examining interindividual differences in the neural mechanisms underlying self-control. Human brain mapping 33, 2768-2784.

DiPatrizio, N.V., Astarita, G., Schwartz, G., Li, X., Piomelli, D., 2011. Endocannabinoid signal in the gut controls dietary fat intake. Proceedings of the National Academy of Sciences of the United States of America 108, 12904-12908.

DiPatrizio, N.V., Joslin, A., Jung, K.M., Piomelli, D., 2013. Endocannabinoid signaling in the gut mediates preference for dietary unsaturated fats. FASEB Journal 27, 2513-2520.

DiPatrizio, N.V., Simansky, K.J., 2008. Activating parabrachial cannabinoid CB1 receptors selectively stimulates feeding of palatable foods in rats. The Journal of Neuroscience 28, 9702-9709.

Doolan, K.J., Breslin, G., Hanna, D., Murphy, K., Gallagher, A.M., 2014. Visual attention to food cues in obesity: An eye-tracking study. Obesity 22, 2501-2507.

Droste, S.M., Saland, S.K., Schlitter, E.K., Rodefer, J.S., 2010. AM 251 differentially effects food-maintained responding depending on food palatability. Pharmacology Biochemistry and Behavior 95, 443-448.

Dykes, J., Brunner, E.J., Martikainen, P.T., Wardle, J., 2004. Socioeconomic gradient in body size and obesity among women: The role of dietary restraint, disinhibition and hunger in the whitehall II study. International Journal of Obesity and Related Metabolic Disorders 28, 262-268.

Engeli, S., Bohnke, J., Feldpausch, M., Gorzelniak, K., Janke, J., Batkai, S., Pacher, P., Harvey-White, J., Luft, F.C., Sharma, A.M., Jordan, J., 2005. Activation of the peripheral endocannabinoid system in human obesity. Diabetes 54, 2838-2843.

Engeli, S., Heusser, K., Janke, J., Gorzelniak, K., Bátkai, S., Pacher, P., Harvey-White, J., Luft, F.C., Jordan, J., 2008. Peripheral endocannabinoid system activity in patients treated with sibutramine. Obesity 16, 11351137.

Farrimond, J.A., Whalley, B.J., Williams, C.M., 2012. Cannabinol and cannabidiol exert opposing effects on rat feeding patterns. Psychopharmacology 223, 117-129. 
Fattore, L., Fratta, W., 2010. How important are sex differences in cannabinoid action? British Journal of Pharmacology 160, 544-548.

Faulconbridge, L.F., Ruparel, K., Loughead, J., Allison, K.C., Hesson, L.A., Fabricatore, A.N., Rochette, A., Ritter, S., Hopson, R.D., Sarwer, D.B., Williams, N.N., Geliebter, A., Gur, R.C., Wadden, T.A., 2016. Changes in neural responsivity to highly palatable foods following roux-en-y gastric bypass, sleeve gastrectomy, or weight stability: An fMRI study. Obesity 24, 1054-1060.

Fedoroff, I., Polivy, J., Herman, C.P., 2003. The specificity of restrained versus unrestrained eaters' responses to food cues: General desire to eat, or craving for the cued food? Appetite 41, 7-13.

Filbey, F.M., Myers, U.S., DeWitt, S., 2012. Reward circuit function in high bmi individuals with compulsive overeating: Similarities with addiction. Neuroimage 63, 1800-1806.

Finlayson, G., King, N., Blundell, J.E., 2007. Is it possible to dissociate 'liking' and 'wanting' for foods in humans? A novel experimental procedure. Physiology and Behavior 90, 36-42.

Finlayson, G., King, N., Blundell, J., 2008. The role of implicit wanting in relation to explicit liking and wanting for food: Implications for appetite control. Appetite 50, 120-127.

Fogel, J.S., Kelly, T.H., Westgate, P.M., Lile, J.A., 2017. Sex differences in the subjective effects of oral $\delta 9$-thc in cannabis users. Pharmacology Biochemistry and Behavior 152, 44-51.

Foltin, R.W., Fischman, M.W., Byrne, M.F., 1988. Effects of smoked marijuana on food intake and body weight of humans living in a residential laboratory. Appetite 11, 1-14.

Frank, R.A., Van der Klaauw, N.J., 1994. The contribution of chemosensory factors to individual differences in reported food preferences. Appetite 22, 101-123.

Frank, S., Heinze, J.M., Fritsche, A., Linder, K., Von Feilitzsch, M., Königsrainer, A., Häring, H.U., Veit, R., Preissl, H., 2016. Neuronal food reward activity in patients with type 2 diabetes with improved glycemic control after bariatric surgery. Diabetes Care 39, 1311-1317.

Frasnelli, J., Hummel, C., Bojanowski, V., Warr, J., Gerber, J., Hummel, T., 2015. Food-related odors and the reward circuit: Functional mri. Chemosensory Perception 8, 192-200.

Friedman, A.N., Kim, J., Kaiser, S., Pedersen, T.L., Newman, J.W., Watkins, B.A., 2016. Association between plasma endocannabinoids and appetite in hemodialysis patients: A pilot study. Nutrition research 36, 658-662. Fulton, S., 2010. Appetite and reward. Frontiers in Neuroendocrinology 31, 85-103.

Gatta-Cherifi, B., Matias, I., Vallee, M., Tabarin, A., Marsicano, G., Piazza, P.V., Cota, D., 2012. Simultaneous postprandial deregulation of the orexigenic endocannabinoid anandamide and the anorexigenic peptide yy in obesity. International journal of obesity 36, 880-885.

Geiselman, P.J., Anderson, A.M., Dowdy, M.L., West, D.B., Redmann, S.M., Smith, S.R., 1998. Reliability and validity of a macronutrient self-selection paradigm and a food preference questionnaire. Physiology and Behavior 63, 919-928.

Geliebter, A., 2013. Neuroimaging of gastric distension and gastric bypass surgery. Appetite 71, 459-465.

Giesen, J.C.A.H., Havermans, R.C., Douven, A., Tekelenburg, M., Jansen, A., 2010. Will work for snack food: The association of BMI and snack reinforcement. Obesity 18, 966-970.

Gloy, V.L., Briel, M., Bhatt, D.L., Kashyap, S.R., Schauer, P.R., Mingrone, G., Bucher, H.C., Nordmann, A.J., 2013. Bariatric surgery versus non-surgical treatment for obesity: A systematic review and meta-analysis of randomised controlled trials. BMJ347, f5934.

Green, B.G., Shaffer, G.S., Gilmore, M.M., 1993. Derivation and evaluation of a semantic scale of oral sensation magnitude with apparent ratio properties. Chemical senses 18, 683-702.

Griffioen-Roose, S., Finlayson, G., Mars, M., Blundell, J.E., de Graaf, C., 2010. Measuring food reward and the transfer effect of sensory specific satiety. Appetite 55, 648-655.

Griffioen-Roose, S., Hogenkamp, P.S., Mars, M., Finlayson, G., de Graaf, C., 2012a. Taste of a 24-h diet and its effect on subsequent food preferences and satiety. Appetite 59, 1-8. 
Griffioen-Roose, S., Mars, M., Finlayson, G., Blundell, J.E., De Graaf, C., 2011. The effect of within-meal protein content and taste on subsequent food choice and satiety. British Journal of Nutrition 106, 779-788. Griffioen-Roose, S., Mars, M., Siebelink, E., Finlayson, G., Tomé, D., De Graaf, C., 2012b. Protein status elicits compensatory changes in food intake and food preferences. The American journal of clinical nutrition 95, 3238.

Griffioen-Roose, S., Smeets, P.A.M., Van Den Heuvel, E., Boesveldt, S., Finlayson, G., De Graaf, C., 2014. Human protein status modulates brain reward responses to food cues. The American journal of clinical nutrition 100, 113-122.

Gross, J., Woelbert, E., Zimmermann, J., Okamoto-Barth, S., Riedl, A., Goebel, R., 2014. Value signals in the prefrontal cortex predict individual preferences across reward categories. The Journal of neuroscience 34, 7580-7586.

Guerrieri, R., Nederkoorn, C., Jansen, A., 2008. The interaction between impulsivity and a varied food environment: Its influence on food intake and overweight. International journal of obesity 32, 708-714.

Guinard, J.X., Brun, P., 1998. Sensory-specific satiety: Comparison of taste and texture effects. Appetite 31, 141-157.

Haase, L., Cerf-Ducastel, B., Murphy, C., 2009. Cortical activation in response to pure taste stimuli during the physiological states of hunger and satiety. Neuroimage 44, 1008-1021.

Hammer, H.F., 2012. Medical complications of bariatric surgery: Focus on malabsorption and dumping syndrome. Digestive Diseases 30, 182-186.

Hanlon, E.C., Tasali, E., Leproult, R., Stuhr, K.L., Doncheck, E., de Wit, H., Hillard, C.J., Van Cauter, E., 2015. Circadian rhythm of circulating levels of the endocannabinoid 2-arachidonoylglycerol. The Journal of clinical endocrinology and metabolism 100, 220-226.

Hansen, H.S., 2014. Role of anorectic n-acylethanolamines in intestinal physiology and satiety control with respect to dietary fat. Pharmacological Research 86, 18-25.

Hansen, T.T., Jakobsen, T.A., Nielsen, M.S., Sjödin, A., Le Roux, C.W., Schmidt, J.B., 2016. Hedonic changes in food choices following roux-en-y gastric bypass. Obesity Surgery 26, 1946-1955.

Hare, T.A., Malmaud, J., Rangel, A., 2011. Focusing attention on the health aspects of foods changes value signals in vmpfc and improves dietary choice. Journal of neuroscience 31, 11077-11087.

Hart, C.L., Ward, A.S., Haney, M., Comer, S.D., Foltin, R.W., Fischman, M.W., 2002. Comparison of smoked marijuana and oral 89 -tetrahydrocannabinol in humans. Psychopharmacology 164, 407-415.

Havermans, R.C., 2011. "You say it's liking, i say it's wanting ...". On the difficulty of disentangling food reward in man. Appetite 57, 286-294.

Hendrikse, J.J., Cachia, R.L., Kothe, E.J., McPhie, S., Skouteris, H., Hayden, M.J., 2015. Attentional biases for food cues in overweight and individuals with obesity: A systematic review of the literature. Obesity Reviews 16, 424-432.

Higgs, S., Williams, C.M., Kirkham, T.C., 2003. Cannabinoid influences on palatability: Microstructural analysis of sucrose drinking after $\delta 9$-tetrahydrocannabinol, anandamide, 2-arachidonoyl glycerol and sr141716. Psychopharmacology 165, 370-377.

Hill , A.J., 1986. Investigation of some short-term influences on hunger, satiety and food consumption in man. University of Leeds.

Hill, A.J., Leathwood, P.D., Blundell, J.E., 1987. Some evidence for short-term caloric compensation in normal weight human subjects: The effects of high- and low-energy meals on hunger, food preference and food intake. Human nutrition: applied nutrition 41, 244-257.

Hill, M.N., Miller, G.E., Ho, W.S., Gorzalka, B.B., Hillard, C.J., 2008. Serum endocannabinoid content is altered in females with depressive disorders: A preliminary report. Pharmacopsychiatry 41, 48-53. 
Himes, S.M., Grothe, K.B., Clark, M.M., Swain, J.M., Collazo-Clavell, M.L., Sarr, M.G., 2015. Stop regain: A pilot psychological intervention for bariatric patients experiencing weight regain. Obesity surgery 25, 922927.

Hirose, S., Chikazoe, J., Jimura, K., Yamashita, K., Miyashita, Y., Konishi, S., 2009. Sub-centimeter scale functional organization in human inferior frontal gyrus. Neuroimage 47, 442-450.

Hng, K.N., Ang, Y.S., 2012. Overview of bariatric surgery for the physician. Clinical Medicine 12, 435-440.

Hogenkamp, P.S., Sundbom, M., Nilsson, V.C., Benedict, C., Schioth, H.B., 2015. Patients lacking sustainable long-term weight loss after gastric bypass surgery show signs of decreased inhibitory control of prepotent responses. PLoS One 10, e0119896.

Hopkins, M., Gibbons, C., Caudwell, P., Blundell, J.E., Finlayson, G., 2016. Differing effects of high-fat or high-carbohydrate meals on food hedonics in overweight and obese individuals. The British journal of nutrition, 1-10.

Horder, J., Harmer, C.J., Cowen, P.J., McCabe, C., 2010. Reduced neural response to reward following 7 days treatment with the cannabinoid CB1 antagonist rimonabant in healthy volunteers. International Journal of Neuropsychopharmacology 13, 1103-1113.

Houben, K., Nederkoorn, C., Jansen, A., 2012. Too tempting to resist? Past success at weight control rather than dietary restraint determines exposure-induced disinhibited eating. Appetite 59, 550-555.

Howlett, A.C., 2002. The cannabinoid receptors. Prostaglandins and Other Lipid Mediators 68-69, 619-631.

Hulshof, K.F.A.M., Ocke, M.C., van Rossum, C.T.M., Buurma-Rethans, E.J.M., Brants, H.A.M., Drijvers, J.J.M.M., ter Doest, D., 2004. Results of the national food consumption survey 2003. RIVM, National Institute for Public Health and the Environment.

Hummel, T., Kobal, G., Gudziol, H., Mackay-Sim, A., 2007. Normative data for the "sniffin' sticks" including tests of odor identification, odor discrimination, and olfactory thresholds: An upgrade based on a group of more than 3,000 subjects. European archives of oto-rhino-laryngology 264, 237-243.

Hutcherson, C.A., Plassmann, H., Gross, J.J., Rangel, A., 2012. Cognitive regulation during decision making shifts behavioral control between ventromedial and dorsolateral prefrontal value systems. The Journal of neuroscience 32, 13543-13554.

Ijpma, I., Renken, R.J., Gietema, J.A., Slart, R.H.J.A., Mensink, M.G.J., Lefrandt, J.D., Ter Horst, G.J., Reyners, A.K.L., 2016. Changes in taste and smell function, dietary intake, food preference, and body composition in testicular cancer patients treated with cisplatin-based chemotherapy. Clinical Nutrition.

Jadoon, K.A., Ratcliffe, S.H., Barrett, D.A., Thomas, E.L., Stott, C., Bell, J.D., O’Sullivan, S.E., Tan, G.D., 2016. Efficacy and safety of cannabidiol and tetrahydrocannabivarin on glycemic and lipid parameters in patients with type 2 diabetes: A randomized, double-blind, placebo-controlled, parallel group pilot study. Diabetes Care 39, 1777-1786.

Jager, G., Witkamp, R.F., 2014. The endocannabinoid system and appetite: Relevance for food reward. Nutrition Research Reviews 27, 172-185.

Jiang, T., Soussignan, R., Schaal, B., Royet, J.-P., 2015. Reward for food odors: An fMRI study of liking and wanting as a function of metabolic state and BMI. Social Cognitive and Affective Neuroscience 10, 561-568. Kemps, E., Tiggemann, M., 2015. Approach bias for food cues in obese individuals. Psychology \& Health 30, 370-380

Kenler, H.A., Brolin, R.E., Cody, R.P., 1990. Changes in eating behavior after horizontal gastroplasty and roux-en-y gastric bypass. The American journal of clinical nutrition 52, 87-92.

Kenny, P.J., 2011. Reward mechanisms in obesity: New insights and future directions. Neuron 69, 664-679. Khandekar, N., Berning, B.A., Sainsbury, A., Lin, S., 2015. The role of pancreatic polypeptide in the regulation of energy homeostasis. Molecular and Cellular Endocrinology 418, Part 1, 33-41. 
Killgore, W.D.S., Young, A.D., Femia, L.A., Bogorodzki, P., Rogowska, J., Yurgelun-Todd, D.A., 2003. Cortical and limbic activation during viewing of high- versus low-calorie foods. Neuroimage 19, 1381-1394.

King, J.A., Korb, F.M., Egner, T., 2012. Priming of control: Implicit contextual cuing of top-down attentional set. The Journal of Neuroscience 32, 8192-8200.

Kirkham, T.C., Williams, C.M., Fezza, F., Di Marzo, V., 2002. Endocannabinoid levels in rat limbic forebrain and hypothalamus in relation to fasting, feeding and satiation: Stimulation of eating by 2-arachidonoyl glycerol. British Journal of Pharmacology 136, 550-557.

Kleinloog, D., Roozen, F., De Winter, W., Freijer, J., Van Gerven, J., 2014. Profiling the subjective effects of 89-tetrahydrocannabinol using visual analogue scales. International Journal of Methods in Psychiatric Research, 245-256.

Kober, H., Mende-Siedlecki, P., Kross, E.F., Weber, J., Mischel, W., Hart, C.L., Ochsner, K.N., 2010. Prefrontal-striatal pathway underlies cognitive regulation of craving. Proceedings of the National Academy of Sciences of the United States of America 107, 14811-14816.

Kola, B., Farkas, I., Christ-Crain, M., Wittmann, G., Lolli, F., Amin, F., Harvey-White, J., Liposits, Z., Kunos, G., Grossman, A.B., Fekete, C., Korbonits, M., 2008. The orexigenic effect of ghrelin is mediated through central activation of the endogenous cannabinoid system. PLOS ONE3.

Kowal, M.A., Hazekamp, A., Colzato, L.S., van Steenbergen, H., van der Wee, N.J.A., Durieux, J., Manai, M., Hommel, B., 2015. Cannabis and creativity: Highly potent cannabis impairs divergent thinking in regular cannabis users. Psychopharmacology 232, 1123-1134.

Krebs, R.M., Boehler, C.N., Roberts, K.C., Song, A.W., Woldorff, M.G., 2012. The involvement of the dopaminergic midbrain and cortico-striatal-thalamic circuits in the integration of reward prospect and attentional task demands. Cerebral Cortex 22, 607-615.

Kringelbach, M.L., Stein, A., van Hartevelt, T.J., 2012. The functional human neuroanatomy of food pleasure cycles. Physiology \& Behavior 106, 307-316.

Kroemer, N.B., Krebs, L., Kobiella, A., Grimm, O., Pilhatsch, M., Bidlingmaier, M., Zimmermann, U.S., Smolka, M.N., 2013. Fasting levels of ghrelin covary with the brain response to food pictures. Addiction Biology 18, 855-862.

Kroemer, N.B., Small, D.M., 2016. Fuel not fun: Reinterpreting attenuated brain responses to reward in obesity. Physiology \& Behavior 162, 37-45.

Kruseman, M., Leimgruber, A., Zumbach, F., Golay, A., 2010. Dietary, weight, and psychological changes among patients with obesity, 8 years after gastric bypass. Journal of the American Dietetic Association 110, 527-534.

Kulendran, M., Borovoi, L., Purkayastha, S., Darzi, A., Vlaev, I., 2017. Impulsivity predicts weight loss after obesity surgery. Surgery for Obesity and Related Diseases 13, 1033-1040.

Kunos, G., Tam, J., 2011. The case for peripheral cb(1) receptor blockade in the treatment of visceral obesity and its cardiometabolic complications. British Journal of Pharmacology 163, 1423-1431.

LaBar, K.S., Gitelman, D.R., Parrish, T.B., Kim, Y.H., Nobre, A.C., Mesulam, M.M., 2001. Hunger selectively modulates corticolimbic activation to food stimuli in humans. Behavioral Neuroscience 115, 493-500.

Lapenta, O.M., Sierve, K.D., de Macedo, E.C., Fregni, F., Boggio, P.S., 2014. Transcranial direct current stimulation modulates erp-indexed inhibitory control and reduces food consumption. Appetite 83, 42-48.

Larsen, J.K., Hermans, R.C.J., Engels, R.C.M.E., 2012. Food intake in response to food-cue exposure. Examining the influence of duration of the cue exposure and trait impulsivity. Appetite 58, 907-913.

Laurenius, A., Larsson, I., Melanson, K.J., Lindroos, A.K., Lönroth, H., Bosaeus, I., Olbers, T., 2013. Decreased energy density and changes in food selection following roux-en-y gastric bypass. European Journal of Clinical Nutrition 67, 168-173. 
le Roux, C.W., Bueter, M., Theis, N., Werling, M., Ashrafian, H., Löwenstein, C., Athanasiou, T., Bloom, S.R., Spector, A.C., Olbers, T., Lutz, T.A., 2011. Gastric bypass reduces fat intake and preference. American Journal of Physiology - Regulatory Integrative and Comparative Physiology 301, R1057-R1066.

Lease, H., Hendrie, G.A., Poelman, A.A.M., Delahunty, C., Cox, D.N., 2016. A sensory-diet database: A tool to characterise the sensory qualities of diets. Food Quality and Preference 49, 20-32.

Ledikwe, J.H., Ello-Martin, J., Pelkman, C.L., Birch, L.L., Mannino, M.L., Rolls, B.J., 2007. A reliable, valid questionnaire indicates that preference for dietary fat declines when following a reduced-fat diet. Appetite 49, 74-83.

Leidy, H.J., Lepping, R.J., Savage, C.R., Harris, C.T., 2011. Neural responses to visual food stimuli after a normal vs. Higher protein breakfast in breakfast-skipping teens: A pilot fMRI study. Obesity 19, 2019-2025.

Li-Ling, J., Irving, M., 2001. Therapeutic value of octreotide for patients with severe dumping syndrome--a review of randomised controlled trials. Postgraduate medical journal 77, 441-442.

Li, C.S., Huang, C., Constable, R.T., Sinha, R., 2006. Imaging response inhibition in a stop-signal task: Neural correlates independent of signal monitoring and post-response processing. The Journal of Neuroscience 26, 186-192.

Ligresti, A., De Petrocellis, L., Di Marzo, V., 2016. From phytocannabinoids to cannabinoid receptors and endocannabinoids: Pleiotropic physiological and pathological roles through complex pharmacology. Physiological reviews 96, 1593-1659.

Lowe, M.R., Butryn, M.L., 2007. Hedonic hunger: A new dimension of appetite? Physiology and Behavior 91, 432-439.

Magro, D.O., Geloneze, B., Delfini, R., Pareja, B.C., Callejas, F., Pareja, J.C., 2008. Long-term weight regain after gastric bypass: A 5-year prospective study. Obesity surgery 18, 648-651.

Mallipedhi, A., Prior, S.L., Dunseath, G., Bracken, R.M., Barry, J., Caplin, S., Eyre, N., Morgan, J., Baxter, J.N., O'Sullivan, S.E., Sarmad, S., Barrett, D.A., Bain, S.C., Luzio, S.D., Stephens, J.W., 2015. Changes in plasma levels of $n$-arachidonoyl ethanolamine and n-palmitoylethanolamine following bariatric surgery in morbidly obese females with impaired glucose homeostasis. Journal of Diabetes Research 2015.

Martin, C., Visalli, M., Lange, C., Schlich, P., Issanchou, S., 2014. Creation of a food taste database using an in-home "taste" profile method. Food Quality and Preference 36, 70-80.

Martin, C.K., Rosenbaum, D., Han, H., Geiselman, P.J., Wyatt, H.R., Hill, J.O., Brill, C., Bailer, B., Miller-Iii, B.V., Stein, R., Klein, S., Foster, G.D., 2011. Change in food cravings, food preferences, and appetite during a low-carbohydrate and low-fat diet. Obesity 19, 1963-1970.

Mathes, C.M., Spector, A.C., 2012. Food selection and taste changes in humans after roux-en-y gastric bypass surgery: A direct-measures approach. Physiology and Behavior 107, 476-483.

Matias, I., Gatta-Cherifi, B., Tabarin, A., Clark, S., Leste-Lasserre, T., Marsicano, G., Piazza, P.V., Cota, D., 2012. Endocannabinoids measurement in human saliva as potential biomarker of obesity. PLoS ONE7.

Mattes, R.D., 1997. Physiologic responses to sensory stimulation by food. Journal of the American Dietetic Association 97, 406-413.

Mattes, R.D., Engelman, K., Shaw, L.M., Elsohly, M.A., 1994a. Cannabinoids and appetite stimulation. Pharmacology Biochemistry and Behavior 49, 187-195.

Mattes, R.D., Shaw, L.M., Engelman, K., 1994b. Effects of cannabinoids (marijuana) on taste intensity and hedonic ratings and salivary flow of adults. Chemical Senses 19, 125-140.

McCaffery, J.M., Haley, A.P., Sweet, L.H., Phelan, S., Raynor, H.A., Del Parigi, A., Cohen, R., Wing, R.R., 2009. Differential functional magnetic resonance imaging response to food pictures in successful weight-loss maintainers relative to normal-weight and obese controls. The American journal of clinical nutrition 90, 928934. 
McCrickerd, K., Forde, C.G., 2016. Sensory influences on food intake control: Moving beyond palatability. Obesity Reviews 17, 18-29.

McPartland, J.M., Duncan, M., Di Marzo, V., Pertwee, R.G., 2015. Are cannabidiol and delta(9)tetrahydrocannabivarin negative modulators of the endocannabinoid system? A systematic review. British journal of pharmacology 172, 737-753.

Meek, C.L., Lewis, H.B., Reimann, F., Gribble, F.M., Park, A.J., 2016. The effect of bariatric surgery on gastrointestinal and pancreatic peptide hormones. Peptides 77, 28-37.

Meillon, S., Miras, A.D., Roux, C.W.1., 2013. Gastric bypass surgery alters food preferences through changes in the perception of taste. Clinical Practice 10, 471-479.

Mela, D.J., 2006. Eating for pleasure or just wanting to eat? Reconsidering sensory hedonic responses as a driver of obesity. Appetite 47, 10-17.

Mennella, I., Ferracane, R., Zucco, F., Fogliano, V., Vitaglione, P., 2015. Food liking enhances the plasma response of 2-arachidonoylglycerol and of pancreatic polypeptide upon modified sham feeding in humans. The Journal of nutrition 145, 2169-2175.

Meule, A., Lutz, A.P., Vogele, C., Kubler, A., 2014. Impulsive reactions to food-cues predict subsequent food craving. Eating behaviors 15, 99-105.

Miras, A.D., Jackson, R.N., Jackson, S.N., Goldstone, A.P., Olbers, T., Hackenberg, T., Spector, A.C., Le Roux, C.W., 2012. Gastric bypass surgery for obesity decreases the reward value of a sweet-fat stimulus as assessed in a progressive ratio task. The American journal of clinical nutrition 96, 467-473.

Mohanty, A., Gitelman, D.R., Small, D.M., Mesulam, M.M., 2008. The spatial attention network interacts with limbic and monoaminergic systems to modulate motivation-induced attention shifts. Cerebral Cortex 18, 2604-2613.

Montecucco, F., Lenglet, S., Quercioli, A., Burger, F., Thomas, A., Lauer, E., da Silva, A.R., Mach, F., Vuilleumier, N., Bobbioni-Harsch, E., Golay, A., Schindler, T.H., Pataky, Z., 2015. Gastric bypass in morbid obese patients is associated with reduction in adipose tissue inflammation via n-oleoylethanolamide (oea)mediated pathways. Thrombosis and haemostasis 113, 838-850.

Monteleone, A.M., Di Marzo, V., Monteleone, P., Dalle Grave, R., Aveta, T., Ghoch, M.E., Piscitelli, F., Volpe, U., Calugi, S., Maj, M., 2016. Responses of peripheral endocannabinoids and endocannabinoid-related compounds to hedonic eating in obesity. European Journal of Nutrition 55, 1799-1805.

Monteleone, P., Piscitelli, F., Scognamiglio, P., Monteleone, A.M., Canestrelli, B., Di Marzo, V., Maj, M., 2012. Hedonic eating is associated with increased peripheral levels of ghrelin and the endocannabinoid 2arachidonoyl-glycerol in healthy humans: A pilot study. Journal of Clinical Endocrinology and Metabolism 97, E917-E924.

Monteleone, P., Scognamiglio, P., Monteleone, A.M., Perillo, D., Canestrelli, B., Maj, M., 2013. Gastroenteric hormone responses to hedonic eating in healthy humans. Psychoneuroendocrinology 38, 1435-1441.

Nakamura, Y., Ikuta, T., 2017. Caudate-precuneus functional connectivity is associated with obesity preventive eating tendency. Brain connectivity 7, 211-217.

Nasser, J., 2001. Taste, food intake and obesity. Obesity Reviews 2, 213-218.

Nederkoorn, C., Guerrieri, R., Havermans, R.C., Roefs, A., Jansen, A., 2009. The interactive effect of hunger and impulsivity on food intake and purchase in a virtual supermarket. International Journal of Obesity 33, 905-912.

Nederkoorn, C., Smulders, F.T., Havermans, R.C., Roefs, A., Jansen, A., 2006. Impulsivity in obese women. Appetite 47, 253-256.

Nielsen, M.S., Christensen, B.J., Ritz, C., Rasmussen, S., Hansen, T.T., Bredie, W.L.P., Le Roux, C.W., Sjödin, A., Schmidt, J.B., 2017. Roux-en-y gastric bypass and sleeve gastrectomy does not affect food preferences when assessed by an ad libitum buffet meal. Obesity Surgery, 1-7. 
Nijs, I.M.T., Muris, P., Euser, A.S., Franken, I.H.A., 2010. Differences in attention to food and food intake between overweight/obese and normal-weight females under conditions of hunger and satiety. Appetite 54, 243-254.

Niki, M., Jyotaki, M., Yoshida, R., Yasumatsu, K., Shigemura, N., DiPatrizio, N.V., Piomelli, D., Ninomiya, Y., 2015. Modulation of sweet taste sensitivities by endogenous leptin and endocannabinoids in mice. The Journal of physiology 593, 2527-2545.

Nummenmaa, L., Hirvonen, J., Hannukainen, J.C., Immonen, H., Lindroos, M.M., Salminen, P., Nuutila, P., 2012. Dorsal striatum and its limbic connectivity mediate abnormal anticipatory reward processing in obesity. PLoS One 7, e31089.

O'Brien, L.D., Wills, K.L., Segsworth, B., Dashney, B., Rock, E.M., Limebeer, C.L., Parker, L.A., 2013. Effect of chronic exposure to rimonabant and phytocannabinoids on anxiety-like behavior and saccharin palatability. Pharmacology Biochemistry and Behavior 103, 597-602.

Ochner, C.N., Kwok, Y., Conceição, E., Pantazatos, S.P., Puma, L.M., Carnell, S., Teixeira, J., Hirsch, J., Geliebter, A., 2011. Selective reduction in neural responses to high calorie foods following gastric bypass surgery. Annals of surgery 253, 502-507.

Ochner, C.N., Laferrère, B., Afifi, L., Atalayer, D., Geliebter, A., Teixeira, J., 2012a. Neural responsivity to food cues in fasted and fed states pre and post gastric bypass surgery. Neuroscience Research 74, 138-143.

Ochner, C.N., Stice, E., Hutchins, E., Afifi, L., Geliebter, A., Hirsch, J., Teixeira, J., 2012b. Relation between changes in neural responsivity and reductions in desire to eat high-calorie foods following gastric bypass surgery. Neuroscience 209, 128-135.

Pastor, A., Fernández-Aranda, F., Fit, M., Jiménez-Murcia, S., Botella, C., Fernández-Real, J.M., Frühbeck, G., Tinahones, F.J., Fagundo, A.B., Rodriguez, J., Agüera, Z., Langohr, K., Casanueva, F.F., De La Torre, R., 2016.

A lower olfactory capacity is related to higher circulating concentrations of endocannabinoid 2arachidonoylglycerol and higher body mass index in women. PLoS ONE 11.

Patriarca, L., Magerowski, G., Alonso-Alonso, M., 2017. Functional neuroimaging in obesity. Current Opinion in Endocrinology, Diabetes and Obesity 24, 154-159.

Pedersen, S.D., 2013. The role of hormonal factors in weight loss and recidivism after bariatric surgery. Gastroenterology Research and Practice 2013.

Pepino, M.Y., Bradley, D., Eagon, J.C., Sullivan, S., Abumrad, N.A., Klein, S., 2014. Changes in taste perception and eating behavior after bariatric surgery-induced weight loss in women. Obesity 22, E13-20.

Pertwee, R.G., 2006. The pharmacology of cannabinoid receptors and their ligands: An overview. International Journal of Obesity 30, S13-S18.

Pertwee, R.G., 2008. The diverse $\mathrm{CB}(1)$ and $\mathrm{CB}(2)$ receptor pharmacology of three plant cannabinoids: $\Delta(9)-$ tetrahydrocannabinol, cannabidiol and $\delta(9)$-tetrahydrocannabivarin. British Journal of Pharmacology 153, 199-215.

Phelan, S., Hassenstab, J., McCaffery, J.M., Sweet, L., Raynor, H.A., Cohen, R.A., Wing, R.R., 2011. Cognitive interference from food cues in weight loss maintainers, normal weight, and obese individuals. Obesity 19, 6973.

Pi-Sunyer, F.X., Aronne, L.J., Heshmati, H.M., Devin, J., Rosenstock, J., 2006. Effect of rimonabant, a cannabinoid-1 receptor blocker, on weight and cardiometabolic risk factors in overweight or obese patients: Rio-north america: A randomized controlled trial. JAMA 295, 761-775.

Piazza, P.V., Cota, D., Marsicano, G., 2017. The cb1 receptor as the cornerstone of exostasis. Neuron 93, 12521274.

Power, M.L., Schulkin, J., 2008. Anticipatory physiological regulation in feeding biology: Cephalic phase responses. Appetite 50, 194-206. 
Price, M., Higgs, S., Lee, M., 2015. Self-reported eating traits: Underlying components of food responsivity and dietary restriction are positively related to bmi. Appetite 95, 203-210.

Primeaux, S.D., Tzeng, T.H., Allerton, T.D., Chiang, M.C., Cosentino, G., Dubin, R.L., Varughese, A., Moore, R., Geiselman, P.J., Greenway, F.L., Uwaifo, G.I., 2015. Differences in short-term food preferences following vertical sleeve gastrectomy and roux-en-y gastric bypass surgery. Obesity Research \& Clinical Practice 9, 628632.

Puzziferri, N., Zigman, J.M., Thomas, B.P., Mihalakos, P., Gallagher, R., Lutter, M., Carmody, T., Lu, H., Tamminga, C.A., 2016. Brain imaging demonstrates a reduced neural impact of eating in obesity. Obesity 24, 829-836.

Quercioli, A., Montecucco, F., Pataky, Z., Thomas, A., Ambrosio, G., Staub, C., Di Marzo, V., Ratib, O., Mach, F., Golay, A., Schindler, T.H., 2013. Improvement in coronary circulatory function in morbidly obese individuals after gastric bypass-induced weight loss: Relation to alterations in endocannabinoids and adipocytokines. European Heart Journal 34, 2063-2073.

Ramaekers, M.G., Luning, P.A., Lakemond, C.M.M., Van Boekel, M.A.J.S., Gort, G., Boesveldt, S., 2016. Food preference and appetite after switching between sweet and savoury odours in women. PLOS ONE 11.

Rasmussen, E.B., Reilly, W., Buckley, J., Boomhower, S.R., 2012. Rimonabant reduces the essential value of food in the genetically obese zucker rat: An exponential demand analysis. Physiology and Behavior 105, 734741.

Richardson, J.T.E., 2011. Eta squared and partial eta squared as measures of effect size in educational research. Educational Research Review 6, 135-147.

Riedel, G., Fadda, P., McKillop-Smith, S., Pertwee, R.G., Platt, B., Robinson, L., 2009. Synthetic and plantderived cannabinoid receptor antagonists show hypophagic properties in fasted and non-fasted mice. British Journal of Pharmacology 156, 1154-1166.

Rigamonti, A.E., Piscitelli, F., Aveta, T., Agosti, F., De Col, A., Bini, S., Cella, S.G., Di Marzo, V., Sartorio, A., 2015. Anticipatory and consummatory effects of (hedonic) chocolate intake are associated with increased circulating levels of the orexigenic peptide ghrelin and endocannabinoids in obese adults. Food \& Nutrition Research 59.

RIVM, 2011. Nevo online version, 3.0 ed, Bilthoven.

Rogers, P.J., 1990. Why a palatability construct is needed. Appetite 14, 167-170.

Rolls, B.J., Rolls, E.T., Rowe, E.A., Sweeney, K., 1981. Sensory specific satiety in man. Physiology and Behavior 27, 137-142.

Rubino, F., Gagner, M., Gentileschi, P., Kini, S., Fukuyama, S., Feng, J., Diamond, E., 2004. The early effect of the roux-en-y gastric bypass on hormones involved in body weight regulation and glucose metabolism. Annals of Surgery 240, 236-242.

Ryden, A., Sullivan, M., Torgerson, J.S., Karlsson, J., Lindroos, A.K., Taft, C., 2003. Severe obesity and personality: A comparative controlled study of personality traits. International Journal of Obesity and Related Metabolic Disorders 27, 1534-1540.

Saelens, B.E., Epstein, L.H., 1996. Reinforcing value of food in obese and non-obese women. Appetite 27, 4150 .

Saliba, J., Wattacheril, J., Abumrad, N.N., 2009. Endocrine and metabolic response to gastric bypass. Current opinion in clinical nutrition and metabolic care 12, 515-521.

Scharmuller, W., Ubel, S., Ebner, F., Schienle, A., 2012. Appetite regulation during food cue exposure: A comparison of normal-weight and obese women. Neuroscience letters 518, 106-110.

Schofield, W.N., 1985. Predicting basal metabolic rate, new standards and review of previous work. Human nutrition. Clinical nutrition 39 Suppl 1, 5-41. 
Scholtz, S., Miras, A.D., Chhina, N., Prechtl, C.G., Sleeth, M.L., Daud, N.M., Ismail, N.A., Durighel, G., Ahmed, A.R., Olbers, T., Vincent, R.P., Alaghband-Zadeh, J., Ghatei, M.A., Waldman, A.D., Frost, G.S., Bell, J.D., le Roux, C.W., Goldstone, A.P., 2014. Obese patients after gastric bypass surgery have lower brainhedonic responses to food than after gastric banding. Gut 63, 891-902.

Schonberg, T., Bakkour, A., Hover, A.M., Mumford, J.A., Poldrack, R.A., 2014. Influencing food choices by training: Evidence for modulation of frontoparietal control signals. Journal of cognitive neuroscience 26, 247268.

Schrieks, I.C., Ripken, D., Stafleu, A., Witkamp, R.F., Hendriks, H.F.J., 2015. Effects of mood inductions by meal ambiance and moderate alcohol consumption on endocannabinoids and n-acylethanolamines in humans: A randomized crossover trial. PLOS ONE 10.

Schultes, B., Ernst, B., Wilms, B., Thurnheer, M., Hallschmid, M., 2010. Hedonic hunger is increased in severely obese patients and is reduced after gastric bypass surgery. The American journal of clinical nutrition 92, 277-283.

Schussler, P., Kluge, M., Yassouridis, A., Dresler, M., Uhr, M., Steiger, A., 2012. Ghrelin levels increase after pictures showing food. Obesity 20, 1212-1217.

Schutz, H.G., Cardello, A.V., 2001. A labeled affective magnitude (lam) scale for assessing food liking/disliking. Journal of Sensory Studies 16, 117-159.

Scott, T.R., 2008. Orosensory control of feeding, Appetite and food intake. CRC Press, pp. 113-131.

Sebastian, A., Gerdes, B., Feige, B., Kloppel, S., Lange, T., Philipsen, A., Tebartz van Elst, L., Lieb, K., Tuscher, O., 2012. Neural correlates of interference inhibition, action withholding and action cancelation in adult adhd. Psychiatry research 202, 132-141.

Shepherd, G.M., 2006. Smell images and the flavour system in the human brain. Nature 444, 316-321.

Sim-Selley, L.J., Schechter, N.S., Rorrer, W.K., Dalton, G.D., Hernandez, J., Martin, B.R., Selley, D.E., 2006. Prolonged recovery rate of cb1 receptor adaptation after cessation of long-term cannabinoid administration. Molecular Pharmacology 70, 986-996.

Sjöström, L., 2013. Review of the key results from the swedish obese subjects (sos) trial - a prospective controlled intervention study of bariatric surgery. Journal of Internal Medicine 273, 219-234.

Small, D.M., Zatorre, R.J., Dagher, A., Evans, A.C., Jones-Gotman, M., 2001. Changes in brain activity related to eating chocolate: From pleasure to aversion. Brain 124, 1720-1733.

Smeets, P.A., Kroese, F.M., Evers, C., de Ridder, D.T., 2013. Allured or alarmed: Counteractive control responses to food temptations in the brain. Behavioural brain research 248, 41-45.

Smeets, P.A.M., Erkner, A., De Graaf, C., 2010. Cephalic phase responses and appetite. Nutrition Reviews 68, 643-655.

Smit, E., Crespo, C.J., 2001. Dietary intake and nutritional status of us adult marijuana users: Results from the third national health and nutrition examination survey. Public Health Nutrition 4, 781-786.

Sorokowska, A., Albrecht, E., Haehner, A., Hummel, T., 2015. Extended version of the "sniffin' sticks" identification test: Test-retest reliability and validity. Journal of Neuroscience Methods 243, 111-114.

Stice, E., Spoor, S., Bohon, C., Small, D.M., 2008a. Relation between obesity and blunted striatal response to food is moderated by taqla1 gene. Science 322, 449-452.

Stice, E., Spoor, S., Bohon, C., Veldhuizen, M.G., Small, D.M., 2008b. Relation of reward from food intake and anticipated food intake to obesity: A functional magnetic resonance imaging study. Journal of abnormal psychology 117, 924-935.

Stice, E., Yokum, S., Burger, K.S., Epstein, L.H., Small, D.M., 2011. Youth at risk for obesity show greater activation of striatal and somatosensory regions to food. The Journal of neuroscience 31, 4360-4366. 
Stice, E., Yokum, S., Burger, K.S, Epstein, L.H., Smolen, A., 2012. Multilocus genetic composite reflecting dopamine signaling capacity predicts reward circuitry responsivity. The Journal of neuroscience 32, 1009310100 .

Stoeckel, L.E., Cox, J.E., Cook, E.W., 3rd, Weller, R.E., 2007. Motivational state modulates the hedonic value of food images differently in men and women. Appetite 48, 139-144.

Stoppel, C.M., Boehler, C.N., Strumpf, H., Heinze, H.-J., Hopf, J.-M., Schoenfeld, M.A., 2011. Neural processing of reward magnitude under varying attentional demands. Brain Research 1383, 218-229.

Sugerman, H.J., Starkey, J.V., Birkenhauer, R., 1987. A randomized prospective trial of gastric bypass versus vertical banded gastroplasty for morbid obesity and their effects on sweets versus non-sweets eaters. Annals of Surgery 205, 613-624.

Svane, M.S., Jorgensen, N.B., Bojsen-Moller, K.N., Dirksen, C., Nielsen, S., Kristiansen, V.B., Torang, S., Wewer Albrechtsen, N.J., Rehfeld, J.F., Hartmann, B., Madsbad, S., Holst, J.J., 2016. Peptide yy and glucagonlike peptide-1 contribute to decreased food intake after roux-en-y gastric bypass surgery. International journal of obesity (2005) 40, 1699-1706.

Takemura, H., Samejima, K., Vogels, R., Sakagami, M., Okuda, J., 2011. Stimulus-dependent adjustment of reward prediction error in the midbrain. PLoS One 6, e28337.

Tart, C.T., 1970. Marijuana intoxication: Common experiences. Nature 226, 701-704.

Teff, K.L., Mattes, R.D., Engelman, K., 1991. Cephalic phase insulin release in normal weight males: Verification and reliability. American Journal of Physiology - Endocrinology and Metabolism 261, E430-E436. Terrazzino, S., Berto, F., Carbonare, M.D., Fabris, M., Guiotto, A., Bernardini, D., Leon, A., 2004. Stearoylethanolamide exerts anorexic effects in mice via down-regulation of liver stearoyl-coenzyme a desaturase-1 mrna expression. FASEB Journal 18, 1580-1582.

Thirlby, R.C., Bahiraei, F., Randall, J., Drewnoski, A., 2006. Effect of roux-en-y gastric bypass on satiety and food likes: The role of genetics. Journal of Gastrointestinal Surgery 10, 270-277.

Tschöp, M., Wawarta, R., Riepl, R.L., Friedrich, S., Bidlingmaier, M., Landgraf, R., Folwaczny, C., 2001. Postprandial decrease of circulating human ghrelin levels. Journal of Endocrinological Investigation 24, RC19RC21.

Tudge, L., Williams, C., Cowen, P.J., McCabe, C., 2015. Neural effects of cannabinoid cb1 neutral antagonist tetrahydrocannabivarin on food reward and aversion in healthy volunteers. International Journal of Neuropsychopharmacology 18, pyu094.

Ullrich, J., Ernst, B., Wilms, B., Thurnheer, M., Schultes, B., 2013. Roux-en y gastric bypass surgery reduces hedonic hunger and improves dietary habits in severely obese subjects. Obesity Surgery 23, 50-55.

Vainik, U., Dagher, A., Dube, L., Fellows, L.K., 2013. Neurobehavioural correlates of body mass index and eating behaviours in adults: A systematic review. Neuroscience and Biobehavioral Reviews 37, 279-299.

van den Heuvel, O.A., Van Gorsel, H.C., Veltman, D.J., Van Der Werf, Y.D., 2013. Impairment of executive performance after transcranial magnetic modulation of the left dorsal frontal-striatal circuit. Human brain mapping 34, 347-355.

van der Laan, L.N., de Ridder, D.T., Viergever, M.A., Smeets, P.A., 2014. Activation in inhibitory brain regions during food choice correlates with temptation strength and self-regulatory success in weight-concerned women. Frontiers in neuroscience 8, 308.

van der Laan, L.N., de Ridder, D.T.D., Viergever, M.A., Smeets, P.A.M., 2011. The first taste is always with the eyes: A meta-analysis on the neural correlates of processing visual food cues. Neuroimage 55, 296-303.

Van der Laan, L.N., De Ridder, D.T.D., Viergever, M.A., Smeets, P.A.M., 2012. Appearance matters: Neural correlates of food choice and packaging aesthetics. PLOS ONE7, e41738. 
van der Meer, L., Groenewold, N.A., Nolen, W.A., Pijnenborg, M., Aleman, A., 2011. Inhibit yourself and understand the other: Neural basis of distinct processes underlying theory of mind. Neuroimage 56, 23642374.

van Duijvenvoorde, A.C., Op de Macks, Z.A., Overgaauw, S., Gunther Moor, B., Dahl, R.E., Crone, E.A., 2014. A cross-sectional and longitudinal analysis of reward-related brain activation: Effects of age, pubertal stage, and reward sensitivity. Brain and cognition 89, 3-14.

Van Gaal, L., Pi-Sunyer, X., Despres, J.P., McCarthy, C., Scheen, A., 2008. Efficacy and safety of rimonabant for improvement of multiple cardiometabolic risk factors in overweight/obese patients: Pooled 1-year data from the rimonabant in obesity (rio) program. Diabetes Care 31 Suppl 2, S229-240.

Van Gaal, L.F., Rissanen, A.M., Scheen, A.J., Ziegler, O., Rossner, S., 2005. Effects of the cannabinoid-1 receptor blocker rimonabant on weight reduction and cardiovascular risk factors in overweight patients: 1 year experience from the rio-europe study. The Lancet 365, 1389-1397.

Van Hell, H.H., Bossong, M.G., Jager, G., Kahn, R.S., Ramsey, N.F., 2011. Methods of the pharmacological imaging of the cannabinoid system (phics) study: Towards understanding the role of the brain endocannabinoid system in human cognition. International Journal of Methods in Psychiatric Research 20, 10-27.

Van Strien, T., Frijters, J.E.R., Bergers, G.P.A., Defares, P.B., 1986. The dutch eating behavior questionnaire (debq) for assessment of restrained, emotional, and external eating behavior. International Journal of Eating Disorders 5, 295-315.

Vaughn, L.K., Denning, G., Stuhr, K.L., de Wit, H., Hill, M.N., Hillard, C.J., 2010. Endocannabinoid signalling: Has it got rhythm? British Journal of Pharmacology 160, 530-543.

Viskaal Van Dongen, M., Van Den Berg, M.C., Vink, N., Kok, F.J., De Graaf, C., 2012. Taste-nutrient relationships in commonly consumed foods. British Journal of Nutrition 108, 140-147.

Volkow, N.D., Wang, G.J., Baler, R.D., 2011. Reward, dopamine and the control of food intake: Implications for obesity. Trends in cognitive sciences 15, 37-46.

Volkow, N.D., Wang, G.J., Telang, F., Fowler, J.S., Thanos, P.K., Logan, J., Alexoff, D., Ding, Y.S., Wong, C., Ma, Y., Pradhan, K., 2008. Low dopamine striatal d2 receptors are associated with prefrontal metabolism in obese subjects: Possible contributing factors. Neuroimage 42, 1537-1543.

Vollm, B., Richardson, P., McKie, S., Elliott, R., Deakin, J.F., Anderson, I.M., 2006. Serotonergic modulation of neuronal responses to behavioural inhibition and reinforcing stimuli: An fmri study in healthy volunteers. The European journal of neuroscience 23, 552-560.

Vuorinen, A.-L., Strahilevitz, M.A., Wansink, B., Safer, D.L., 2016. Shifts in the enjoyment of healthy and unhealthy behaviors affect short- and long-term postbariatric weight loss. Bariatric Surgical Practice and Patient Care 12, 35-42.

Walter, C., Ferreirós, N., Bishay, P., Geisslinger, G., Tegeder, I., Lötsch, J., 2013. Exogenous delta9tetrahydrocannabinol influences circulating endogenous cannabinoids in humans. Journal of Clinical Psychopharmacology.

Ward, M., Prachand, V., 2009. Surgical treatment of obesity. Gastrointestinal Endoscopy 70, 985-990.

Weygandt, M., Mai, K., Dommes, E., Ritter, K., Leupelt, V., Spranger, J., Haynes, J.D., 2015. Impulse control in the dorsolateral prefrontal cortex counteracts post-diet weight regain in obesity. Neuroimage 109, 318-327. Wierucka-Rybak, M., Wolak, M., Bojanowska, E., 2014. The effects of leptin in combination with a cannabinoid receptor 1 antagonist, am 251, or cannabidiol on food intake and body weight in rats fed a highfat or a free-choice high sugar diet. Journal of physiology and pharmacology 65, 487-496.

Wiley, J.L., Burston, J.J., Leggett, D.C., Alekseeva, O.O., Razdan, R.K., Mahadevan, A., Martin, B.R., 2005. Cb 1 cannabinoid receptor-mediated modulation of food intake in mice. British Journal of Pharmacology 145, 293-300 
Witteman, B.J., Edwards-Teunissen, K., Hopman, W.P., Jebbink, M.C., Masclee, A.A., Lamers, C.B., Jansen, J.B., 1994. Nutrient-specific effects of modified sham feeding on pancreatic polypeptide release. European Journal of Clinical Nutrition 48, 556-560.

Wittmann, B.C., Schott, B.H., Guderian, S., Frey, J.U., Heinze, H.J., Duzel, E., 2005. Reward-related fMRI activation of dopaminergic midbrain is associated with enhanced hippocampus-dependent long-term memory formation. Neuron 45, 459-467.

Wren, A.M., Small, C.J., Ward, H.L., Murphy, K.G., Dakin, C.L., Taheri, S., Kennedy, A.R., Roberts, G.H., Morgan, D.G.A., Ghatei, M.A., Bloom, S.R., 2000. The novel hypothalamic peptide ghrelin stimulates food intake and growth hormone secretion. Endocrinology 141, 4325-4328.

Yeomans, M.R., 2006. Olfactory influences on appetite and satiety in humans. Physiology \& Behavior 87, 800804.

Yoshida, R., Ohkuri, T., Jyotaki, M., Yasuo, T., Horio, N., Yasumatsu, K., Sanematsu, K., Shigemura, N., Yamamoto, T., Margolskee, R.F., Ninomiya, Y., 2010. Endocannabinoids selectively enhance sweet taste. Proceedings of the National Academy of Sciences of the United States of America 107, 935-939.

Zhang, B., Tian, D., Yu, C., Li, M., Zang, Y., Liu, Y., Walter, M., 2015. Altered baseline brain activity differentiates regional mechanisms subserving biological and psychological alterations in obese men. Scientific reports $5,11563$.

Zhu, Y., Hsu, W.H., Hollis, J.H., 2014. Modified sham feeding of foods with different macronutrient compositions differentially influences cephalic change of insulin, ghrelin and nmr-based metabolomic profiles. Physiology \& Behavior.

Zoon, H.F., de Graaf, C., Boesveldt, S., 2016. Food odours direct specific appetite. Foods 5, 12.

Zoon, H.F.A., He, W., de Wijk, R.A., de Graaf, C., Boesveldt, S., 2014. Food preference and intake in response to ambient odours in overweight and normal-weight females. Physiology \& Behavior 133, 190-196.

Zotev, V., Krueger, F., Phillips, R., Alvarez, R.P., Simmons, W.K., Bellgowan, P., Drevets, W.C., Bodurka, J., 2011. Self-regulation of amygdala activation using real-time fmri neurofeedback. PLoS One 6, e24522. 


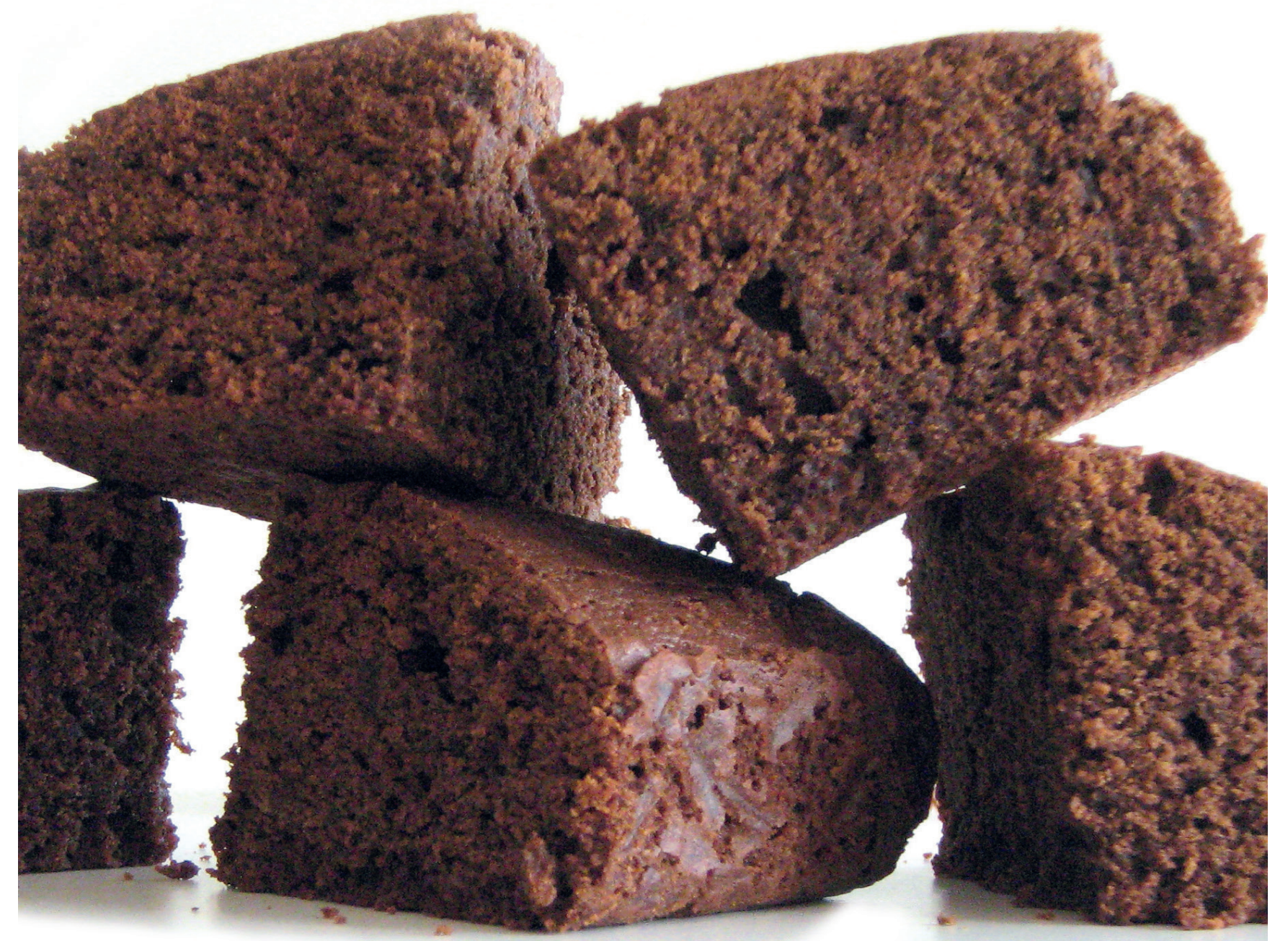


ET CETERA 


\section{Summary}

Food reward is an important driver of food intake and triggers consumption of foods for pleasure, so-called hedonic eating, even in the absence of any energy deficits. Hedonic eating can trigger overeating and may therefore lead to obesity. Given the rise in obesity rates and the health risks associated with being obese, hedonic eating and food reward are important phenomena to study. This thesis aimed to add on to the existing knowledge on food reward. The phenomenon was approached from a behavioural, sensory and (neuro)physiological perspective in healthy, lean and in obese gastric bypass populations.

For the behavioural perspective, the main outcome measure used in this thesis was food preferences. To be able to study food preferences for four macronutrient and two taste categories, a new food preference task was developed. In chapter 2, the development and validation of the Macronutrient and Taste Preference Ranking Task (MTPRT) were described. The MTPRT uses a ranking method to determine preferences for four macronutrient (high-carbohydrate, high-fat, high-protein, low-energy) and two taste (sweet and savoury) categories.

For the sensory and physiological perspective, focus was put on the endocannabinoid system (ECS): a neuromodulatory system that plays a role in food reward. To gain more insight into this role, the effect of ECS modulation with pharmacological challenges on sensory perception of sweet taste and on food preferences were studied, as well as endocannabinoid responses to food intake. In chapter 3 it was shown that inhaling Cannabis with low doses of $\Delta 9$-tetrahydrocannabinol (THC) and cannabidiol (CBD) does not alter sweet taste intensity perception and liking in humans, nor does it affect food preferences. Vice versa, in chapter 4 it was found that liking of a food taste does not affect endocannabinoid responses to food intake, after controlling for expectations. When palatability of the food is unknown until the first bite, response of endocannabinoids, ghrelin and pancreatic polypeptide did not differ between a palatable and a neutral food across anticipatory, consummatory and post-ingestive phases of food intake. Endocannabinoid and ghrelin plasma concentrations decreased after food intake, which suggests an orexigenic function for endocannabinoids.

In chapters 5, 6 and 7, studies with patients who underwent Roux-en-Y gastric bypass surgery were described. These studies were intended to gain more insight into alterations in food reward in relation to (morbid) obesity and in response to surgical treatment by RYGB surgery.

First, in chapter 5 food preferences were assessed before, and at two months and one year after RYGB. It was shown that patients have decreased preference for high-carbohydrate and highfat foods, and increased preference for low-energy foods after compared with before surgery. In addition, liking ratings for the high-carbohydrate and high-fat foods were decreased after RYGB surgery, whereas liking of low-energy products changed minimally. Potential mechanisms behind these alterations in food preferences include changes in neural processing of food cues and changes in appetite-related gut hormones.

In chapter 6, it was shown that alterations in food preferences after RYGB surgery are indeed related to changes in neural activation in response to food cues. With regards to the appetiterelated hormones it was shown that plasma concentrations of the endocannabinoid anandamide were increased after compared with before surgery. Plasma concentrations of other 
endocannabinoids and ghrelin did not change. Moreover, changes in endocannabinoid or ghrelin concentrations did not correlate with changes in food preferences or neural response to food cues. Together, these results suggest that changes in neural processing of food cues contribute to changes in food preferences towards low-energy foods, and provide a first indication that the endocannabinoid system does not seem to play a role in this process.

To gain more insight into behavioural responses to food cues, a response-inhibition paradigm was used in chapter 7 , in which response-inhibition to high-energy and low-energy food cues was assessed during brain imaging. The behavioural data did not show differences in performance when comparing before and two months after RYGB surgery. The brain imaging data showed that activation in reward-related brain areas was decreased in response to both high- and low-energy food pictures after RYGB surgery. Also, prefrontal brain areas were more activated in response to the high-energy pictures, which suggests improved response inhibition.

In conclusion, the findings in this thesis show that modulating the ECS with low doses of THC and CBD does not influence sweet taste perception and liking and food preferences, and vice versa, food taste liking in the absence of expectations does not affect endocannabinoid responses to food intake. With regards to RYGB surgery it was uncovered that changes in food preferences after RYGB surgery are related to altered brain reward processing, but no relation with changes in endocannabinoid tone was found. The success of RYGB surgery and the changes in food choice might in part be caused by an improved inhibitory response to high-energy foods. 


\section{Samenvatting in het Nederlands}

De belonende waarde van voedsel is een belangrijke motivatie voor voedselinname. Deze beloning leidt tot eten omdat het lekker is - zogenaamd hedonisch eten - zelfs als er geen energietekort is. Hedonisch eten kan leiden tot overeten, wat obesitas tot gevolg kan hebben. Gezien de toename in obesitas en de gezondheidsrisico's die met obesitas geassocieerd zijn, is het belangrijk om onderzoek te doen naar hedonisch eten en voedselbeloning. Deze thesis had als doel om bij te dragen aan de kennis over voedselbeloning. Dit fenomeen is benaderd vanuit een gedrags-, sensorisch en (neuro)fysiologisch perspectief in gezonde, dunne en in obese bariatrische chirurgie populaties.

Voor het gedragsperspectief waren voedselvoorkeuren de belangrijkste uitkomstmaat. Om voedselvoorkeuren voor vier macronutriënten en twee smaken te kunnen meten is tijdens dit project een nieuwe methode ontwikkeld. In hoofdstuk 2 is de ontwikkeling en validatie van de Macronutrient and Taste Preference Ranking Task (MTPRT; Nederlands: macronutriënt- en smaakvoorkeurrankingtaak) beschreven. De MTPRT gebruikt een rankingmethode om voorkeuren voor vier macronutriënten (hoog-koolhydraat, hoog-vet, hoog-eiwit, en laag-energie) en twee smaken (zoet en hartig) te meten.

Voor het sensorische en het fysiologische perspectief lag de focus op het endocannabinoïde systeem (ECS): een belangrijk biologisch systeem dat een rol speelt bij voedselbeloning. Om meer inzicht in deze rol te krijgen is tijdens dit project gekeken naar zowel het effect van farmacologische modulatie van het ECS op sensorische perceptie van zoete smaak, en op voedselvoorkeuren, als naar de endocannabinoïde reactie op voedselinname. Hoofdstuk 3 laat zien dat het inhaleren van Cannabis met lage doseringen THC en cannabidiol geen effect heeft op de waarneming van zoete smaakintensiteit en het lekker vinden van zoete smaak, en ook niet op voedselvoorkeuren. Andersom, in hoofdstuk 4 blijkt dat het lekker vinden van een voedingsmiddel geen effect heeft op de reactie van endocannabinoïden op voedselinname, als gecontroleerd wordt voor verwachtingen. Als onbekend is hoe lekker het voedingsmiddel is tot de eerste hap wordt genomen, dan is er geen verschil in de reactie van endocannabinoïden, ghreline en pancreatisch polypeptide op een lekker of een neutraal voedingsmiddel tijdens anticipatie, consumptie en post-ingestive fasen van voedselinname. Plasmaconcentraties van endocannabinoïden en ghreline daalden na voedselinname, wat duidt op een hongeropwekkende functie voor endocannabinoïden.

In hoofdstukken 5, 6 en 7 worden studies beschreven met patiënten die een Roux-en-Y gastric bypass (RYGB) operatie ondergaan. De bedoeling van deze studies is om meer inzicht te krijgen in veranderingen in voedselbeloning in relatie tot (morbide) obesitas en in reactie op chirurgische behandeling door RYGB operatie.

Eerst zijn in hoofdstuk $\mathbf{5}$ voedselvoorkeuren gemeten op twee weken voor, twee maanden na en een jaar na de operatie. Na de operatie hebben patiënten een verminderde voorkeur voor hoog-koolhydraat en hoog-vet voedingsmiddelen, en een verhoogde voorkeur voor laag-energie voedingsmiddelen in vergelijking met voor de operatie. Daarnaast worden de hoog-koolhydraat en hoog-vet voedingsmiddelen na de operatie minder lekker gevonden, terwijl het lekker vinden van de laag-energie voedingsmiddelen minimaal veranderde. Mogelijke mechanismen achter de 
veranderingen in voedselvoorkeuren zijn veranderingen in neurale verwerking van voedselcues en veranderingen in honger-gerelateerde darmhormonen.

Hoofdstuk 6 toont aan dat veranderingen in voedselvoorkeuren inderdaad gerelateerd zijn aan veranderingen in neurale activatie in reactie op voedselcues. Wat betreft de honger-gerelateerde darmhormonen is aangetoond dat de plasmaconcentratie van de endocannabinoïde anandamide na operatie hoger was dan voor de operatie. Plasmaconcentraties van andere endocannabinoïden en ghreline veranderden niet. Veranderingen in plasmaconcentraties van endocannabinoïden en ghreline correleerden niet met veranderingen in voedselvoorkeuren of neurale reacties op voedingscues. Samengenomen suggereren deze resultaten dat veranderingen in neurale verwerking van voedselcues bijdragen aan veranderingen in voedselvoorkeuren richting laag-energie voedingsmiddelen, en ze zijn een eerste indicatie dat het ECS geen rol speelt in dit proces.

Om meer inzicht te krijgen in de gedragsreactie op voedselcues is in hoofdstuk 7 een reactieinhibitie paradigma gebruikt. Neurale activatie tijdens reactie-inhibitie op hoog-energie en laagenergie voedselcues is gemeten. De gedragsdata liet geen verschil zien in prestatie voor operatie en twee maanden na operatie. The hersendata liet zien dat activatie in beloningsgerelateerde hersengebieden na operatie is verlaagd in reactie op zowel hoog-energie als laag-energie voedselcues. Daarnaast zijn prefrontale hersengebieden meer geactiveerd in reactie op hoog-energie voedselcues na operatie in vergelijking met voor operatie. Dit wijst op verbeterde reactie-inhibitie.

In conclusie: de bevindingen in deze thesis laten zien dat het moduleren van het ECS met lage doseringen THC en CBD geen invloed heeft op de waarneming en het lekker vinden van zoete smaak, en op voedselvoorkeuren. Andersom, de aangenaamheid van voedselsmaak in afwezigheid van verwachtingen heeft geen invloed op de endocannabinoïde reactie op voedselinname. Met betrekking tot bariatrische chirurgie is aangetoond dat veranderingen in voedselvoorkeuren gerelateerd zijn aan veranderingen in verwerking van beloning in het brein, maar niet aan veranderingen in endocannabinoïden. Het succes van RYGB operatie en de veranderingen in voedselkeuze kunnen gedeeltelijk veroorzaakt worden door verbeterde reactie-inhibitie op hoogenergie voedsel. 


\section{Dankwoord}

Het is klaar! Het resultaat van 4,5 jaar onderzoek is gebundeld in dit boekje. Dit had ik nooit alleen voor elkaar gekregen en ik wil iedereen bedanken die een bijdrage heeft geleverd.

Om te beginnen mijn co-promotor en promotoren. Gerry, dankjewel voor je vertrouwen in mij om dit project uit te voeren. Ik heb veel geleerd van onze discussies over mijn onderzoek en je kritische blik op het veld. Daarnaast kijk ik met veel plezier terug op de leuke reizen die we hebben gemaakt. Renger, ik bewonder je onuitputtelijke positiviteit en de manier waarop je altijd denkt in mogelijkheden. Dankjewel voor je ideeën om mijn onderzoek nog beter te maken en je feedback op mijn papers. Kees, met jouw kennis op het gebied van sensoriek en eetgedrag heb je een belangrijke bijdrage geleverd aan mijn promotie-onderzoek. Dankjewel voor onze gesprekken over voeding, mijn onderzoek en het publicatieproces.

Daarnaast waren er nog vier mensen lid van het projectteam. Paul, dankjewel voor het meedenken over studiedesign en resultaten, en je feedback op papers. Henk, jij was mijn eerste begeleider bij TNO. Bedankt voor je interesse in het project, ook toen je niet meer mijn begeleider was. Marijn, jij nam het stokje over en introduceerde mij verder bij TNO. Dankjewel voor onze leuke gesprekken. Wilrike, met jou heb ik het einde gehaald. Dankjewel voor je interesse in mij en in alle studies die al draaiden toen jij bij het project kwam. Jouw deur en mailbox stonden altijd voor me open als ik je advies nodig had. Annelies, jij zorgde bij TNO voor de financiële kant van mijn project. Bedankt voor je hulp.

I also would like to take this opportunity to thank the members of my thesis committee for reading this thesis and for being present at my defence: Ben Witteman, Tim Kirkham, David Mela and Laura Steenbergen.

De afgelopen jaren heb ik met verschillende mensen samengewerkt. Ik ben dank verschuldigd aan de mensen van Bedrocan voor hun advies voor de Cannabis-studie. Ik wil hier met name noemen Tjalling Erkelens en Arno Hazekamp. Bedankt voor het meedenken en voor de leuke gesprekken. Dank ook aan Edo en Ignace voor de kans om onderzoek te doen naar de effecten van bariatrische chirurgie. Wendy, Laura, Frieda en Ellen, bedankt voor jullie hulp en inzet rond de werving van de patiënten. Zonder jullie hadden we het niet gekund.

Jet, onze gezamenlijke studie bleek een monsterproject, maar we did it! Ik vond het fijn om met je te samenwerken. Bedankt ook voor je hulp toen ik net begon in het Biotechnion en voor de gezelligheid de afgelopen jaren. Ik ben heel blij dat je straks als paranimf naast mij staat.

Yfke, dankjewel voor onze fijne samenwerking en het samen schrijven van een mooi artikel. Ik vond het gezellig om het laatste jaar ook kamergenootjes te zijn en samen door de laatste schrijffase te gaan.

Daarnaast had ik dit ook niet gekund zonder leuke collega's. Inge, ook jij was in het Biotechnion mijn kamergenoot en altijd bereid om te helpen of een luisterend oor te bieden. Dankjewel voor de gezelligheid. Louise and Vivian, thank you for your interest in my work and nice chats in the office. 
Irene, dankjewel voor je leuke verhalen die voor de nodige afleiding zorgden, en ook voor gezellige bioscoopbezoekjes, koffiemomenten en fietstochten. Ik hoop samen nog veel van de omgeving te zien. Ik ben blij dat jij mijn paranimf wilt zijn.

Guido, zonder jouw drone zou het op onze kamer lang niet zo leuk zijn geweest. Dankjewel voor je steun en luisterende oor in mijn laatste schrijffase.

Ook de andere kamergenootjes in Helix, Ilse, Kamalita en Rachelle, dankjulliewel voor de gezelligheid en adviezen.

Ya, my cannabinoid-buddy. Thank you for teaching me about endocannabinoid-analyses and exchanging literature tips. You were also my roommate at the ICRS. I enjoyed spending time together in Baveno and Bukovina. Michiel, jij was ook onmisbaar voor de endocannabinoïdanalyses. Ik bewonder je enorme kennis op dat gebied. Dankjewel ook voor de leuke gesprekken tijdens de ICRS, ik ga ze zeker missen.

Astrid, Marlou, Ellen, Elly en Liesbeth, bedankt voor de gezellige etentjes. Anouk, Agnes en Maaike, bedankt voor het brainstormen over stellingen en het uitwisselen van tips rond de laatste loodjes. Sanne, Monica, Markus, Swetlana, Victoire, Korrie, Janet, Apple, Patricia, Elbrich, Roelien, Eva, Paulina, Mariëlle, Jocelijn, Klaske, Nikkie, Jvalini, Rogier, Wout, Miranda, bedankt voor jullie interesse in mijn project, gezellige lunches, gesprekken en leuke reizen.

Henriëtte, Jantien, Diane, Mechteld, Rianne en Anita, bedankt voor jullie hulp bij het inplannen van mijn studies, bloedafnames, en advies voor in het lab. Zonder jullie was het niet gelukt om de studies met bloedafnames te draaien. Dione, bedankt voor je hulp bij het werven van deelnemers. Dankjewel Els, Pauline en Desiree voor het uitzoeken van recepten en bestellen van alle boodschappen. Didi, Gea en Jasmijn, bedankt voor het inplannen van de nodige afspraken met het projectteam en regelen van allerhande zaken.

Ook bij TNO en TNO Triskelion heb ik de nodige hulp gehad. Richard, Frederique, Maarten, Jan en Gerard, bedankt voor jullie hulp met de LCMS. Dina, Ilse, Angelique, Esther, Evita, Harm, Irene, Kim, Mariska, Marlous, Mehdi, Petra, Rianne, Bedankt voor jullie hulp, de lunches en gezelligheid op het lab.

Tijdens mijn $\mathrm{PhD}$ heb ik een aantal studenten begeleid bij hun thesis. Nora, Liselore, Tim, Kristel, Michele, Annemiek, Erwin, Dimitra, Anouk and Rachelle: Thank you for your hard work and inspiring conversations about science and non-science things.

Naast het werk, was er gelukkig ook tijd voor ontspanning. Lieve vrienden en familie, bedankt dat jullie er altijd voor mij zijn.

Jacob, Sinoë en Suzanne, het is elke keer een feestje om met jullie een escaperoom te doen. Dankjewel Bart en Sietske, Thomas en Marieke, Jeroen, Myriam en Ruben, Lukas, David en Lydia, Dennis en Marieke, voor alle etentjes en spelletjes. Groeigroepers, Bijbelstudie-ers en FJW-ers, dankjewel voor de nodige afleiding en mooie gesprekken. 
Familie en schoonfamilie, dankjewel voor alle shopsessies en gezelligheid op verjaardagen, feestdagen of gewoon zomaar. Ik vind het altijd leuk om jullie te zien.

Lieve Marlies, dankjewel voor je interesse in wat ik doe en voor alle leuke zussenmomenten. Ik ben supertrots op je.

Lieve mama, dankjewel dat jouw deur altijd voor me open staat. Het is heel fijn om af en toe thuis in Naarden te kunnen komen.

Lieve Steef, met jou is de wereld mooier. Dankjewel dat je er altijd voor me bent, en dat je alle hoogtepunten met me meeviert. Ik hoop nog veel samen te vieren en leuke dingen te doen.

Suzanne. 


\begin{abstract}
About the author
Suzanne de Bruijn was born on September 6th, 1989 in Naarden, the Netherlands. After completing secondary school at Goois Lyceum in Bussum, she went on to study pharmacy at Utrecht University. During her BSc, she completed a minor on Cognition and wrote a thesis entitled "low level tactile sensitivity in Anorexia Nervosa". After her BSc she completed the research master programme Neuroscience and Cognition, for which she completed two internships, one on body representations in Anorexia Nervosa and one on memory processes.

In 2013, Suzanne was appointed as a PhD candidate in the chair groups Sensory Science and Eating Behaviour, and Nutrition and Pharmacology. Her research focussed on behavioural and (neuro)physiological aspects of food reward, as described in this thesis. Suzanne conducted several clinical studies, for which she collaborated with different companies and hospitals.

During her $\mathrm{PhD}$, Suzanne joined the educational program of the graduate school VLAG. She presented her research at several (inter)national conferences and participated in courses. She was involved in teaching and supervision of BSc and MSc students during their thesis projects. Next to this, she was a member of the NutriScope committee, which included updating protocols and guidelines on how to conduct clinical trials at the division of Human Nutrition.
\end{abstract}




\section{List of publications}

\section{Peer reviewed publications}

de Bruijn, S.E.M., de Graaf, C., Witkamp, R.F., Jager, G. (2017) Explorative placebo-controlled double-blind intervention study with low doses of inhaled THC and CBD reveals no effect on sweet taste intensity perception and liking in humans. Cannabis and Cannabinoid Research. 2(1):114-122

de Bruijn, S.E.M., de Vries, Y.C., de Graaf, C., Boesveldt, S., Jager, G. (2017) The reliability and validity of the Macronutrient and Taste Preference Ranking Task: a new method to measure food preferences. Food Quality and Preference, 57:32-40

Keizer, A., de Bruijn, S.E.M., Smeets, M.A.M., Dijkerman, H.C, Postma, A. (2013) Walking through apertures: Do you know what you are doing during body-scaled action? Perception, 42(5):583-585

\section{Submitted papers}

de Bruijn, S.E.M., Witkamp, R.F., de Graaf, C., Pasman, W.J., Smeets, P.A.M., Jager, G. No difference in satiety hormone and endocannabinoid response to palatable vs neutral brownie intake. Submitted.

Zoon, H.F.A., de Bruijn, S.E.M., Jager, G., Smeets, P.A.M., de Graaf, C., Janssen, I.M.C., Schijns, W., Aarts, E.O., Boesveldt, S. Altered neural responsivity to food cues in relation to food preferences, but not appetite-related hormone concentrations after RYGB-surgery. Submitted.

Zoon, H.F.A., de Bruijn, S.E.M., Jager, G., Smeets, P.A.M., de Graaf, C., Janssen, I.M.C., Schijns, W., Deden, L.N., Boesveldt, S. Altered neural inhibition responses to food cues after Roux-en-Y Gastric Bypass. Submitted.

\section{Papers in preparation for submission}

de Bruijn, S.E.M., Zoon, H.F.A., de Graaf, C., Boesveldt, S., Jager, G. Decreased preference for highcarbohydrate and high-fat foods after Roux-en-Y Gastric Bypass surgery. In preparation for submission.

\section{Abstracts and presentations}

de Bruijn, S.E.M., de Vries, Y.C., de Graaf, C., Boesveldt, S., Jager, G. The reliability and validity of the Macronutrient and Taste Preference Ranking Task: a new method to measure food preferences. Paper presented at the Nutritional Science Days, Heeze, NL. Oral presentation.

de Bruijn, S.E.M., de Graaf, C., Witkamp, R.F., Jager, G. Profiling the endocannabinoid response to hedonic eating. Society for the Study of Ingestive Behavior, 2016, Porto, PT. Poster presentation.

de Bruijn, S.E.M., Andeweg, A.J., de Graaf, C., Witkamp, R.F., Jager, G. Profiling the endocannabinoid response to hedonic eating. International Cannabinoid Research Society, 2016 Bukowina Tatrzanska, PL, Oral presentation.

de Bruijn, S.E.M., Pedrotti, M.D.V., de Graaf, C., Witkamp, R.F., Jager, G. How our mouth feeds our brain: Effects of endocannabinoid modulation on sweet taste perception and liking in humans. Research in Progress Poster Session, Center for Basic and Translational Research on Disorders of the Digestive System, 2015, Rockefeller University, US, Poster presentation. 
de Bruijn, S.E.M., Pedrotti, M.D.V., de Graaf, C., Witkamp, R.F., Jager, G. How our mouth feeds our brain: Effects of endocannabinoid modulation on sweet taste perception and liking in humans. International Cannabinoid Research Society, 2016, Wolfville, CA. Poster presentation.

de Bruijn, S.E.M., Zoon, H.F.A., de Graaf, C., Jager, G. Food preferences after Roux-en-Y Gastric Bypass surgery. Appetite 101, p. 229. British Feeding and Drinking Group, 2015, Wageningen, NL. Poster presentation.

de Bruijn, S.E.M., Zoon, H.F.A., Weitkamp, L., Jager, G., de Graaf, C., Boesveldt, S. Brain reward response to the sight and smell of food. Appetite 83, p. 349. British Feeding and Drinking Group, 2014, Portsmouth, UK. Oral presentation. 


\section{Overview of completed training activities}

\begin{tabular}{|c|c|c|}
\hline \multicolumn{3}{|l|}{ Discipline specific activities } \\
\hline $\begin{array}{l}\text { Course 'Nutriscience, a multifaceted approach to nutrition } \\
\text { research' }\end{array}$ & Wageningen, NL & 2013 \\
\hline 7th Conference on Cannabinoids in Medicine & Cologne, DE & 2013 \\
\hline Nutritional Science Days & Deurne, NL & 2013 \\
\hline Course 'Sensory Perception and Food Preference' & Wageningen, NL & 2013 \\
\hline 38th Annual Meeting of the British Feeding \& Drinking Group & Portsmouth, UK & 2014 \\
\hline 12th Dutch Endo-Neuro-Psycho meeting & Lunteren, NL & 2014 \\
\hline $\begin{array}{l}\text { 24th Annual Symposium of the International Cannabinoid } \\
\text { Research Society }\end{array}$ & Baveno, IT & 2014 \\
\hline Course 'Sensory Evaluation and Food Preferences' & Copenhagen, DK & 2014 \\
\hline $\begin{array}{l}\text { 25th Annual Symposium of the International Cannabinoid } \\
\text { Research Society }\end{array}$ & Wolfville, CA & 2015 \\
\hline 39th Annual Meeting of the British Feeding \& Drinking Group & Wageningen, NL & 2015 \\
\hline $\begin{array}{l}\text { 26th Annual Symposium of the International Cannabinoid } \\
\text { Research Society }\end{array}$ & $\begin{array}{l}\text { Bukowina } \\
\text { Tatrzanska, PL }\end{array}$ & 2016 \\
\hline $\begin{array}{l}\text { 24th Annual meeting of the Society for the Study of Ingestive } \\
\text { Behavior }\end{array}$ & Porto, PT & 2016 \\
\hline Nutritional Science Days & Heeze, NL & 2016 \\
\hline \multicolumn{3}{|l|}{ General courses } \\
\hline VLAG PhD week & Baarlo, NL & 2013 \\
\hline Teaching and supervising thesis students & Wageningen, NL & 2013 \\
\hline Good Clinical Practice & Amersfoort, NL & 2013 \\
\hline PhD Workshop Carousel & Wageningen, NL & 2014 \\
\hline LCMS & Brussel, BE & 2015 \\
\hline Philosophy and Ethics of Food Science and Technology & Wageningen, NL & 2015 \\
\hline National PhDDay & Delft, NL & 2015 \\
\hline Scientific Writing & Wageningen, NL & 2016 \\
\hline Career Perspectives & Wageningen, NL & 2016 \\
\hline \multicolumn{3}{|l|}{ Optionals } \\
\hline Preparation of research proposal & Wageningen, NL & 2013 \\
\hline PhD study tour to USA & USA & 2015 \\
\hline Staff seminars and chair group meetings & Wageningen, NL & 2013-2017 \\
\hline
\end{tabular}




\section{Colophon}

The research described in this thesis has been supported by a research grant from graduate school VLAG (Advanced Studies in Food Technology, Agro-biotechnology, Nutrition and Health Sciences, Wageningen University).

Financial support from Wageningen University for printing this thesis is gratefully acknowledged.

Cover design by Suzanne de Bruijn and Ruben Stoel

Printed by Digiforce - proefschriftmaken.nl Vianen

Copyright (C) S.E.M. de Bruijn, 2017. 